\title{
VARIABILITY STUDY WITH FRIT 510 TO SUPPORT A SECOND TANK 40 DECANT
}

F.C. Raszewski

T.B. Edwards

D.K. Peeler

D.R. Best

I.A. Reamer

R.J. Workman

July 2008

Environmental \& Chemical Process Technology Savannah River National Laboratory Aiken, SC 29808

Prepared for the U.S. Department of Energy Under Contract Number DEAC09-96SR18500

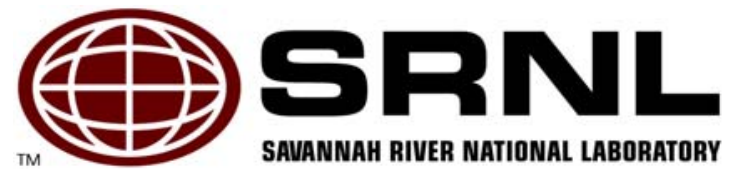


WSRC-STI-2008-00315

Revision 0

\section{DISCLAIMER}

This report was prepared by Washington Savannah River Company (WSRC) for the United States Department of Energy under Contract No. DE-AC09-96SR18500 and is an account of work performed under that contract. Neither the United States Department of Energy, nor WSRC, nor any of their employees makes any warranty, expressed or implied, or assumes any legal liability or responsibility for the accuracy, completeness, or usefulness, of any information, apparatus, or product or process disclosed herein or represents that its use will not infringe privately owned rights. Reference herein to any specific commercial product, process, or service by trademark, name, manufacturer or otherwise does not necessarily constitute or imply endorsement, recommendation, or favoring of same by WSRC or by the United States Government or any agency thereof. The views and opinions of the authors expressed herein do not necessarily state or reflect those of the United States Government or any agency thereof.

\section{Printed in the United States of America \\ Prepared For U.S. Department of Energy}

The Savannah River National Laboratory is operated for the U.S. Department of Energy by Washington Savannah River Company. 
Keywords: SB4, Frit 510,

decant, variability, durability

Retention: permanent

\section{VARIABILITY STUDY WITH FRIT 510 TO SUPPORT A SECOND TANK 40 DECANT}

F.C. Raszewski

T.B. Edwards

D.K. Peeler

D.R. Best

I.A. Reamer

R.J. Workman

July 2008

Environmental \& Chemical Process Technology Savannah River National Laboratory Aiken, SC 29808

Prepared for the U.S. Department of Energy Under Contract Number DEAC09-96SR18500

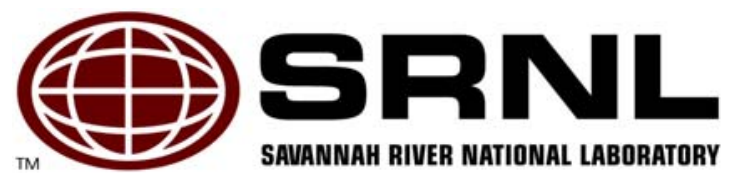




\section{REVIEWS AND APPROVALS}

\section{AUTHORS:}

F.C. Raszewski, Process Engineering Technology

Date

T.B. Edwards, Statistical Consulting Section

Date

D.K. Peeler, Process Engineering Technology

Date

D.R. Best, Process Engineering Technology

Date

I.A. Reamer, Engineering Process Development

Date

R.J. Workman, Engineering Process Development

Date

\section{TECHNICAL REVIEWER:}

K. M. Fox, Process Engineering Technology

Date

\section{APPROVERS:}

J.C. Griffin, Manager, E\&CPT Research Programs

Date

C.C. Herman, Manager, Process Engineering Technology

Date

J.E. Occhipinti, Manager, Waste Solidification Engineering

Date 
WSRC-STI-2008-00315

Revision 0

\section{EXECUTIVE SUMMARY}

Sludge Batch 4 (SB4) is currently being processed in the Defense Waste Processing Facility (DWPF) using Frit 510. The slurry pumps in Tank 40 are experiencing in-leakage of bearing water, which is causing the sludge slurry in Tank 40 to become dilute at a rapid rate. Currently, the DWPF is removing this dilution water by performing caustic boiling during the Sludge Receipt and Adjustment Tank (SRAT) cycle. In order to alleviate prolonged SRAT cycle times, which may eventually impact canister production rates, the Liquid Waste Organization (LWO) performed a $100 \mathrm{~K}$ gallon supernate decant of Tank 40 in April 2008. SRNL performed a supplemental glass variability study to support the April 2008 100K gallon decant incorporating the impact of coupled operations (addition of the Actinide Removal Process (ARP) stream).

Recently LWO requested that SRNL assess the impact of a second decant (up to $100 \mathrm{~K}$ gallon) to the Frit 510-SB4 system. This second decant occurred in June 2008. LWO provided nominal compositions on May 6, 2008 representing Tank 40 prior to the second decant, following the second decant, and the SB4 Heel prior to blending with Tank 51 to constitute SB5. Paper study assessments were performed for these options based on sludge-only and coupled operations processing (ARP addition), as well as possible $\mathrm{Na}_{2} \mathrm{O}$ additions (via $\mathrm{NaOH}$ additions) to both flowsheets. A review of the $\mathrm{ComPro}^{\mathrm{TM}}$ database relative to the compositional region defined by the projections after the second decant coupled with Frit 510 identified only a few glasses with similar glass compositions. These glasses were acceptable from a durability perspective, but did not sufficiently cover the new glass compositional region. Therefore, SRNL recommended that a supplemental variability study be performed to support the June 2008 Tank 40 decant.

Glasses were selected for the variability study based on three sludge compositional projections (sludge-only, coupled and coupled $+2 \mathrm{wt} \% \mathrm{Na}_{2} \mathrm{O}$ ) at waste loadings (WLs) of interest to DWPF $(32 \%, 35 \%$ and 38\%). These nine glasses were fabricated and characterized using chemical composition analysis, X-ray Diffraction (XRD) and the Product Consistency Test (PCT).

All of the glasses that were selected for this study satisfy the Product Composition Control System (PCCS) criteria and are deemed processable and acceptable for the DWPF, except for the SB4VS2-03 (sludge-only at $38 \% \mathrm{WL}$ ) target composition. This glass fails the $\mathrm{T}_{\mathrm{L}}$ criterion and would not be considered processable based on Slurry Mix Evaporator (SME) acceptability decisions.

The durabilities of all of the study glasses (both quenched and $\mathrm{ccc}$ ) are well below that of the normalized leachate for boron (NL [B]) of the reference EA glass $(16.695 \mathrm{~g} / \mathrm{L})$ and are predictable using the current PCCS models. Very little variation exists between the NL [B] of the quenched and ccc versions of the glasses. There is some evidence of a trend toward a less durable glass as WL increases for some of the sludge projections.

Frit 510 is a viable option for the processing of SB4 after a second Tank 40 decant with or without the addition of products from the ARP stream as well as the $2 \mathrm{wt} \% \mathrm{Na}_{2} \mathrm{O}$ addition. The addition of ARP had no negative impacts on the acceptability and predictability of the variability study glasses. 


\section{TABLE OF CONTENTS}

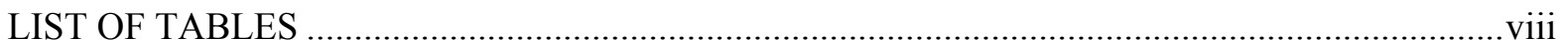

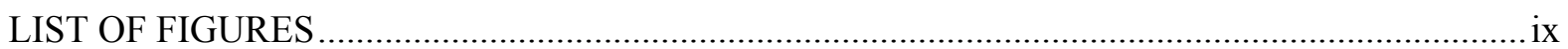

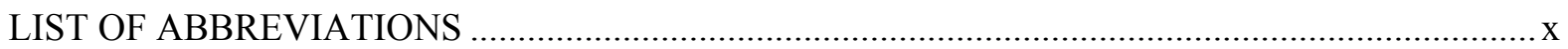

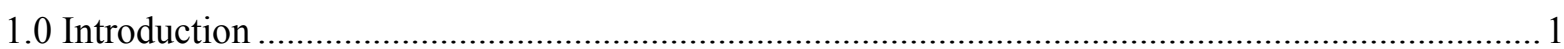

1.1 Glass Selection Strategy for the Second Variability Study..................................................... 1

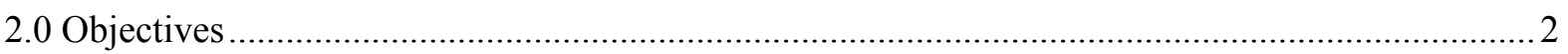

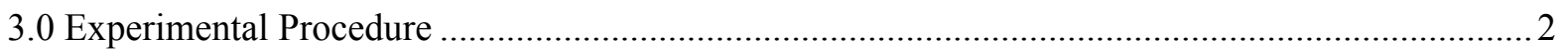

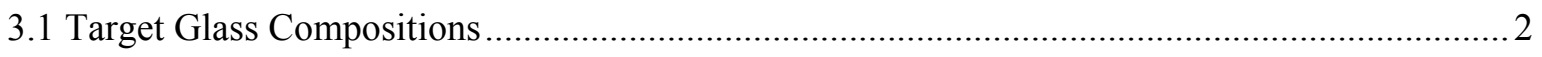

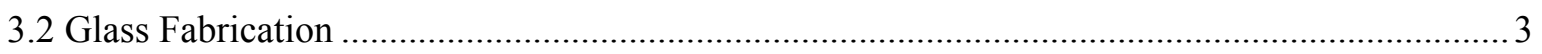

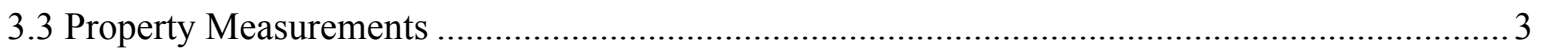

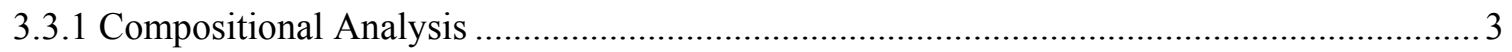

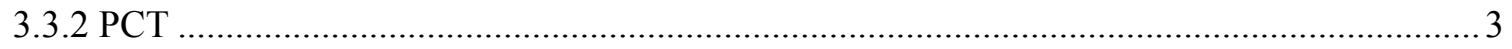

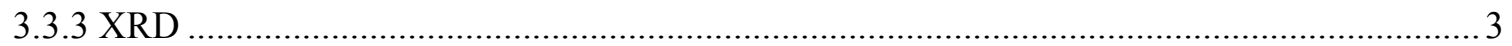

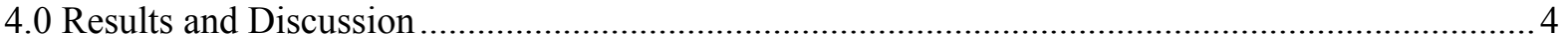

4.1 Statistical Review of the Chemical Composition Measurements .............................................. 4

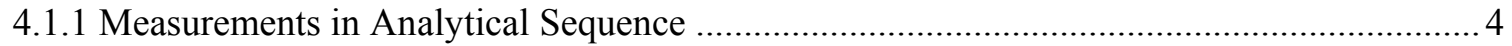

4.1.2 Composition Measurements by Glass Identifier ................................................................. 4

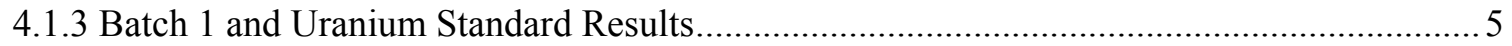

4.1.4 Composition Measurements by Glass Identifier with Targeted Compositions ...................... 6

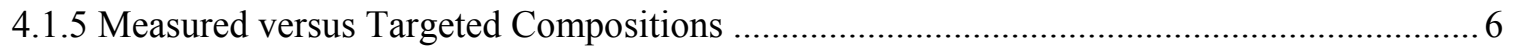

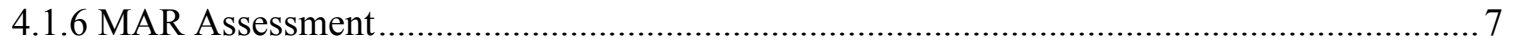

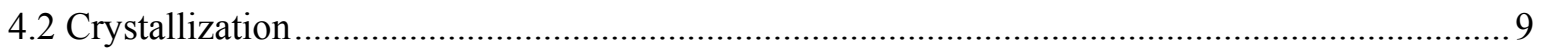

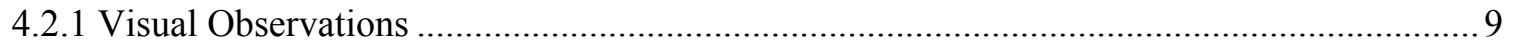

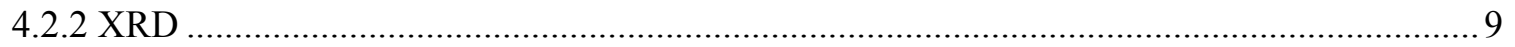

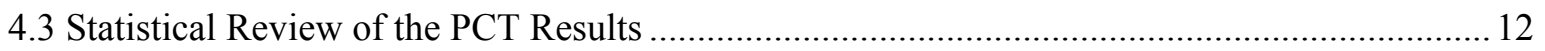

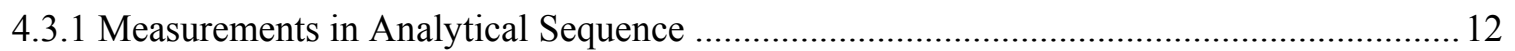

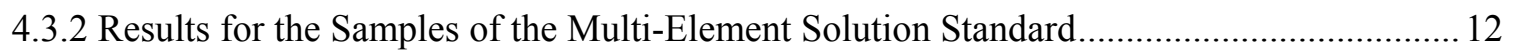

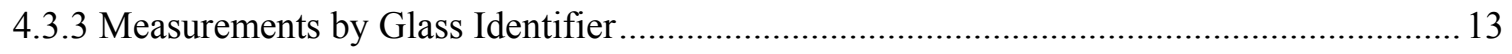

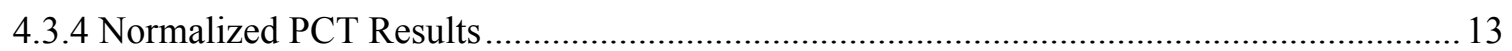

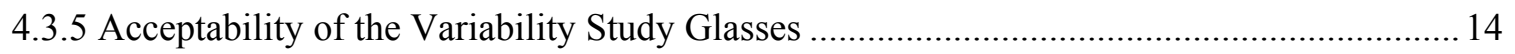

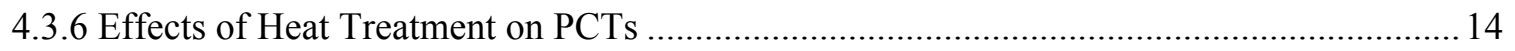

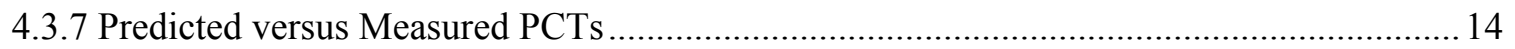


WSRC-STI-2008-00315

Revision 0

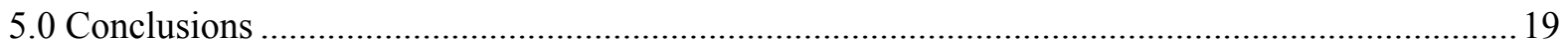

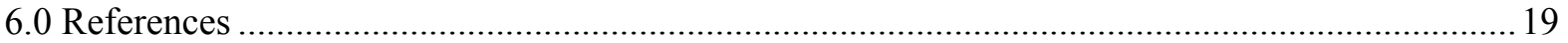




\section{LIST OF TABLES}

Table 1. Target Compositions of the Second Variability Study Glasses............................................ 2

Table 2. Questionable Measurements Eliminated From Further Analysis ......................................... 7

Table 3. Results of the MAR Assessment of SB4 VS Glasses for Measured, Bias Corrected and

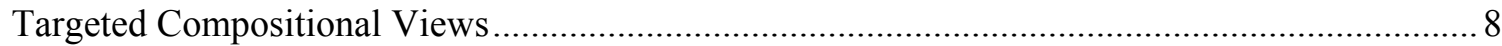

Table 4. Visual Observations of Quenched and CCC Glasses ........................................................ 10

Table 5. Results from Samples of the Multi-Element Solution Standard........................................... 12

Table 6. Normalized PCTs by Glass ID/Compositional View for SB4/Second Decant Glasses with

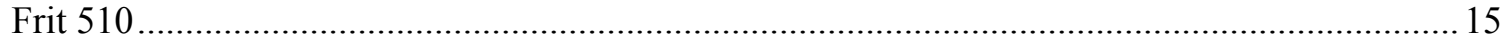




\section{LIST OF FIGURES}

Figure 1. A representative XRD pattern of an amorphous sample. Note: "SB4VAR2" on sample label (upper left hand corner) should be "SB4VS2.".

Figure 2. XRD pattern of the ccc version of the SB4VS2-03 glass (sludge-only at 38\% WL). Note: "SB4VAR2" on sample label (upper left hand corner) should be "SB4VS2."

Figure 3. Log NL[B] versus B del Gp model with a 95\% confidence interval for individual PCTs... 18 


\section{LIST OF ABBREVIATIONS}

\begin{tabular}{|c|c|}
\hline $\mathrm{AD}$ & Analytical Development \\
\hline ANOVA & Analysis of Variance \\
\hline ARM & Approved Reference Material \\
\hline ARP & Actinide Removal Process \\
\hline B Del Gp & $\Delta \mathrm{Gp}$ value for boron \\
\hline bc & Bias-Corrected \\
\hline $\mathrm{ccc}$ & Centerline Canister Cooling \\
\hline $\mathrm{CPC}$ & Chemical Processing Cell \\
\hline DWPF & Defense Waste Processing Facility \\
\hline EA & Environmental Assessment \\
\hline ICP-AES & Inductively Coupled Plasma-Atomic Emission Spectroscopy \\
\hline ID & Identification \\
\hline LM & Lithium Metaborate \\
\hline LWO & Liquid Waste Organization \\
\hline MAR & Measure Acceptability Region \\
\hline $\mathrm{NL}[\mathrm{B}]$ & Normalized Leachate for Boron \\
\hline NL[Li] & Normalized Leachate for Lithium \\
\hline $\mathrm{NL}[\mathrm{Na}]$ & Normalized Leachate for Sodium \\
\hline $\mathrm{NL}[\mathrm{Si}]$ & Normalized Leachate for Silicon \\
\hline PCCS & Product Composition Control System \\
\hline PCT & Product Consistency Test \\
\hline $\mathrm{PF}$ & Sodium Peroxide Fusion \\
\hline ppm & Parts per million \\
\hline PSAL & Process Science Analytical Laboratory \\
\hline SB4 & Sludge Batch 4 \\
\hline SME & Slurry Mix Evaporator \\
\hline SRAT & Sludge Receipt and Adjustment Tank \\
\hline SRNL & Savannah River National Laboratory \\
\hline $\mathrm{T}_{\mathrm{L}}$ Pred & Liquidus Temperature Prediction \\
\hline $\mathrm{U}_{\text {std }}$ & Uranium Standard \\
\hline Visc Pred & Viscosity Prediction \\
\hline WL & Waste Loading \\
\hline XRD & X-ray Diffraction \\
\hline
\end{tabular}


WSRC-STI-2008-00315

Revision 0

\subsection{Introduction}

Sludge Batch 4 (SB4) is currently being processed in the Defense Waste Processing Facility (DWPF) using Frit 510. The slurry pumps in Tank 40 are experiencing in-leakage of bearing water, which is causing the sludge slurry in Tank 40 to become dilute at a rapid rate. Currently, the DWPF is removing this dilution water by performing caustic boiling during the Sludge Receipt and Adjustment Tank (SRAT) cycle. In order to alleviate prolonged SRAT cycle times, which may eventually impact canister production rates, the Liquid Waste Organization (LWO) performed a 100K gallon supernate decant of Tank 40 in April 2008. SRNL performed a supplemental glass variability study to support the April 2008 100K gallon decant incorporating the impact of coupled operations (addition of the Actinide Removal Process (ARP) stream). ${ }^{1}$

Recently LWO requested that SRNL assess the impact of a second decant (up to $100 \mathrm{~K}$ gallon) to the Frit 510-SB4 system. This second decant occurred in June 2008. LWO provided nominal compositions on May 6, 2008 representing Tank 40 prior to the second decant, following the second decant, and the SB4 Heel prior to blending with Tank 51 to constitute SB5. Paper study assessments were performed for these options based on sludge-only and coupled operations, as well as possible $\mathrm{NaOH}$ additions to both flowsheets. ${ }^{\mathrm{a}, 2}$ A review of the ComPro ${ }^{\mathrm{TM}}$ database relative to the compositional region defined by the projections after the second decant coupled with Frit 510 identified only a few historical glasses with similar compositions. These glasses were acceptable from a durability perspective, but did not sufficiently cover the new glass compositional region. Therefore, SRNL recommended that a supplemental variability study be performed to support the June 2008 Tank 40 decant. $^{2}$ This work was carried out under the auspices of a Technical Task Request (TTR) issued by LWO and a Task Technical and Quality Assurance Plan (TT\&QAP).,

\subsection{Glass Selection Strategy for the Second Variability Study}

Three nominal sludge compositions were chosen to be combined with Frit 510 for the experimental variability study:

1. Sludge-only [Tk 40 Post Late Decant $]^{\mathrm{b}}$

2. Coupled ${ }^{c}$ [Tk 40 Post Late Decant $w$ ARP]

3. Coupled $+2 \mathrm{wt} \% \mathrm{Na}_{2} \mathrm{O}$ [Tk 40 Post Late Decant $\left.w \mathrm{ARP}+2 \% \mathrm{Na} 2 \mathrm{O}\right]$

Both options 1 and 2 (sludge-only and coupled) were chosen as bounding conditions for the study. Option 3 (Coupled $+2 \mathrm{wt} \% \mathrm{Na}_{2} \mathrm{O}$ ) was chosen based on the results of the Measurement Acceptability Region (MAR) paper study assessments. ${ }^{2}$ LWO was considering the addition of $\mathrm{NaOH}$ to Tank 40 or in the Chemical Processing Cell (CPC) in order to increase the size of the projected operating window and/or improve melt rate (assuming that melt rate would be reduced by the decant and/or ARP addition). The MAR assessments indicated that the addition of 1 and $2 \mathrm{wt} \% \mathrm{Na}_{2} \mathrm{O}$ to a coupled operations flowsheet was feasible; however, the addition of $3 \mathrm{wt} \% \mathrm{Na}_{2} \mathrm{O}$ caused the system to become limited by nepheline at the upper waste loading. Thus, the $2 \mathrm{wt} \%$ addition was chosen for this study, as the upper waste loading was only limited by liquidus temperature $\left(\mathrm{T}_{\mathrm{L}}\right)$.

\footnotetext{
${ }^{a}$ The projected compositions were provided by LWO on May 6, 2008 (D. Larsen via email communication) and are documented in WSRC-STI-2008-00254. Supplemental washing information was provided by J. Gillam (via D. Larsen email and entitled SB4-5_042808_50\% retention_40 Decant 2 for 51 Wash D_20cpm.xls).

${ }^{\mathrm{b}}$ The terminology in brackets refers to the nomenclature used by LWO.

${ }^{c}$ Compositional information for ARP additions was obtained from X-CLC-S-00113, Rev. 0, Actinide Removal Process Material Balance Calculation with Low Curie Salt Feed, S.G. Subosits, 9/24/2004.
} 
For each of the three sludge options, glasses were selected at waste loadings (WLs) of interest to DWPF $(32 \%, 35 \%$ and $38 \%)$. These nine glasses were fabricated and characterized using chemical composition analysis, X-ray Diffraction (XRD) and the Product Consistency Test (PCT). ${ }^{3,4}$

\subsection{Objectives}

The intent of the experimental portion of the variability study was to demonstrate that the glasses of the Frit 510-modified SB4 compositional region (Options 1-3) after a second Tank 40 decant were both acceptable relative to the Environmental Assessment (EA) reference glass and predictable by the current process control models for durability.

\subsection{Experimental Procedure}

\subsection{Target Glass Compositions}

Target glass compositions of the nine SB4 Tank 40 second decant variability study glasses are presented in Table 1. The nomenclature for the glass identification (ID) can be described as follows: "SB4VS2" refers to Sludge Batch 4 Variability Study 2.

Table 1. Target Compositions of the Second Variability Study Glasses

\begin{tabular}{|c|c|c|c|c|c|c|c|c|c|}
\hline \multirow[b]{2}{*}{ Glass ID } & \multicolumn{3}{|c|}{ Sludge - Only } & \multicolumn{3}{|c|}{ Coupled } & \multicolumn{3}{|c|}{ Coupled + $2 w^{2} \% \mathrm{Na}_{2} \mathrm{O}$} \\
\hline & 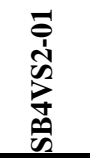 & 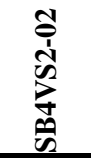 & 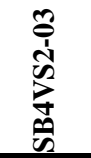 & 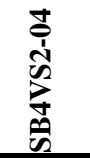 & 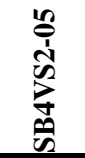 & 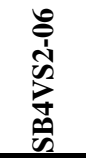 & 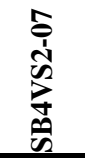 & 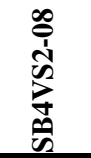 & 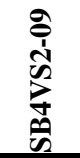 \\
\hline Frit & 510 & 510 & 510 & 510 & 510 & 510 & 510 & 510 & 510 \\
\hline WL & 32 & 35 & 38 & 32 & 35 & 38 & 32 & 35 & 38 \\
\hline $\mathbf{A l}_{2} \mathbf{O}_{3}$ & 8.63 & 9.44 & 10.25 & 8.31 & 9.08 & 9.86 & 8.11 & 8.87 & 9.63 \\
\hline$\overline{\mathbf{B}_{2} \mathbf{O}_{3}}$ & 9.52 & 9.10 & 8.68 & 9.52 & 9.10 & 8.68 & 9.52 & 9.10 & 8.68 \\
\hline $\mathbf{B a O}$ & 0.02 & 0.03 & 0.03 & 0.03 & 0.03 & 0.03 & 0.02 & 0.03 & 0.03 \\
\hline $\mathrm{CaO}$ & 0.95 & 1.04 & 1.13 & 0.92 & 1.01 & 1.09 & 0.90 & 0.98 & 1.07 \\
\hline $\mathrm{Ce}_{2} \mathrm{O}_{3}$ & 0.02 & 0.02 & 0.03 & 0.02 & 0.03 & 0.03 & 0.02 & 0.03 & 0.03 \\
\hline $\mathrm{Cr}_{2} \mathrm{O}_{3}$ & 0.06 & 0.06 & 0.07 & 0.05 & 0.06 & 0.07 & 0.05 & 0.06 & 0.06 \\
\hline $\mathrm{CuO}$ & 0.02 & 0.02 & 0.02 & 0.02 & 0.02 & 0.02 & 0.02 & 0.02 & 0.02 \\
\hline $\mathrm{Fe}_{2} \mathbf{O}_{3}$ & 9.87 & 10.79 & 11.72 & 9.57 & 10.47 & 11.37 & 9.35 & 10.22 & 11.10 \\
\hline $\mathbf{K}_{2} \mathbf{O}$ & 0.00 & 0.00 & 0.00 & 0.00 & 0.00 & 0.00 & 0.00 & 0.00 & 0.00 \\
\hline $\mathbf{L a}_{2} \mathbf{O}_{3}$ & 0.02 & 0.02 & 0.02 & 0.02 & 0.02 & 0.02 & 0.02 & 0.02 & 0.02 \\
\hline $\mathbf{L i}_{2} \mathbf{O}$ & 5.44 & 5.20 & 4.96 & 5.44 & 5.20 & 4.96 & 5.44 & 5.20 & 4.96 \\
\hline MgO & 0.93 & 1.02 & 1.11 & 0.89 & 0.97 & 1.06 & 0.87 & 0.95 & 1.03 \\
\hline $\mathrm{MnO}$ & 1.98 & 2.17 & 2.35 & 1.95 & 2.14 & 2.32 & 1.91 & 2.09 & 2.27 \\
\hline $\mathrm{Na}_{2} \mathrm{O}$ & 10.20 & 10.40 & 10.61 & 10.60 & 10.84 & 11.08 & 11.24 & 11.54 & 11.84 \\
\hline $\mathrm{NiO}$ & 0.55 & 0.60 & 0.65 & 0.55 & 0.60 & 0.65 & 0.53 & 0.58 & 0.63 \\
\hline PbO & 0.02 & 0.02 & 0.02 & 0.02 & 0.02 & 0.03 & 0.02 & 0.02 & 0.02 \\
\hline $\mathrm{SO}_{4}$ & 0.19 & 0.20 & 0.22 & 0.22 & 0.24 & 0.26 & 0.22 & 0.24 & 0.26 \\
\hline $\mathrm{SiO}_{2}$ & 48.53 & 46.51 & 44.50 & 48.49 & 46.47 & 44.46 & 48.47 & 46.45 & 44.43 \\
\hline $\mathrm{TiO}_{2}$ & 0.02 & 0.02 & 0.02 & 0.43 & 0.47 & 0.51 & 0.42 & 0.46 & 0.50 \\
\hline$\overline{\mathrm{U}_{3} \mathbf{O}_{8}}$ & 3.01 & 3.29 & 3.57 & 2.91 & 3.18 & 3.46 & 2.84 & 3.11 & 3.38 \\
\hline $\mathrm{ZnO}$ & 0.00 & 0.00 & 0.00 & 0.00 & 0.00 & 0.00 & 0.00 & 0.00 & 0.00 \\
\hline $\mathrm{ZrO}_{2}$ & 0.03 & 0.03 & 0.03 & 0.03 & 0.03 & 0.03 & 0.03 & 0.03 & 0.03 \\
\hline
\end{tabular}


WSRC-STI-2008-00315

Revision 0

\subsection{Glass Fabrication}

Each variability study glass was prepared from the proper proportions of reagent-grade metal oxides, carbonates, $\mathrm{H}_{3} \mathrm{BO}_{3}$, and salts in $150 \mathrm{~g}$ batches. ${ }^{4}$ The raw materials were thoroughly mixed and placed into a $95 \%$ platinum / $5 \%$ gold, $250 \mathrm{ml}$ crucible. Batched materials were placed into a hightemperature furnace at the target melt temperature of $1150^{\circ} \mathrm{C}$. The crucible was removed from the furnace after an isothermal hold at $1150^{\circ} \mathrm{C}$ for 1 hour. The molten glass was quenched by pouring the liquid onto a clean, stainless steel plate. The glass pour patty was used as a sampling stock for the various property measurements (i.e., chemical composition, durability testing and XRD).

Approximately $25 \mathrm{~g}$ of each glass was heat-treated to simulate cooling along the centerline of a DWPF-type canister to gauge the effects of thermal history on the product performance. ${ }^{5}$ This cooling schedule is referred to as the centerline canister cooling (ccc) curve.

\subsection{Property Measurements}

\subsubsection{Compositional Analysis}

To confirm that the as-fabricated glasses met the target compositions, a representative sample from each glass was submitted to the Process Science Analytical Laboratory (PSAL) for chemical analysis under the auspices of an analytical plan. ${ }^{6}$ Two dissolution methods were utilized in measuring these chemical compositions: samples prepared by lithium metaborate (LM) dissolution were used to measure elemental concentrations of aluminum $(\mathrm{Al})$, barium $(\mathrm{Ba})$, calcium $(\mathrm{Ca})$, cerium $(\mathrm{Ce})$, chromium $(\mathrm{Cr})$, copper $(\mathrm{Cu})$, lanthanum $(\mathrm{La})$, magnesium $(\mathrm{Mg})$, manganese $(\mathrm{Mn})$, sodium $(\mathrm{Na})$, nickel (Ni), lead $(\mathrm{Pb})$, sulfur $(\mathrm{S})$, silicon ( $\mathrm{Si})$, titanium (Ti), uranium (U), and zirconium (Zr), while samples from glasses prepared by peroxide fusion (PF) dissolution were used to measure elemental concentrations of boron (B), iron (Fe), and lithium (Li). For each study glass, measurements were obtained from samples prepared in duplicate by each of these dissolution methods. All of the prepared samples were analyzed (twice for each element of interest) by Inductively Coupled PlasmaAtomic Emission Spectroscopy (ICP-AES) with the instrumentation being re-calibrated between the duplicate analyses. The analytical plan was developed in such a way as to provide the opportunity to evaluate potential sources of bias and error. Glass standards were also intermittently measured to assess the performance of the ICP-AES instrument over the course of these analyses.

\subsubsection{PCT}

A 7-day PCT was performed in triplicate on each quenched and ccc glass to assess chemical durability using Method A of the PCT procedure. ${ }^{7}$ Also included in the experimental test matrix was the EA glass, the Approved Reference Material (ARM) glass, and blanks from the sample cleaning batch. Samples were ground, washed, and prepared according to the standard procedure. The resulting solutions were sampled (filtered and acidified) and analyzed by PSAL under the auspices of an analytical plan. ${ }^{8}$ Samples of a multi-element, standard solution were also included in the analytical plan (as a check on the accuracy of the ICP-AES). Normalized release rates were calculated based on target, measured, and bias-corrected (bc) compositions using the average of the logs of the leachate concentrations.

\subsubsection{XRD}

Representative samples of quenched and ccc glasses were submitted to Analytical Development (AD) for XRD analysis. Samples were analyzed under conditions providing a detection limit of 
approximately $0.5 \mathrm{vol} \%$, i.e. no crystals can be detected if the amount in the sample is less than $\sim 0.5$ vol\%.

\subsection{Results and Discussion}

\subsection{Statistical Review of the Chemical Composition Measurements}

Table A1 in Appendix A provides the elemental concentration measurements derived from the samples prepared using LM and Table A2 in Appendix A provides the measurements derived from the samples prepared using PF. Measured values of the standards (Batch 1 and a uranium standard, $\left.\mathrm{U}_{\text {std }}\right)$ that were included in the PSAL analytical plan along with the study glasses are also provided in these two tables.

The elemental concentrations were converted to oxide concentrations by multiplying the values for each element by the gravimetric factor for the corresponding oxide. During this process, an elemental concentration that was determined to be below the detection limit of the analytical procedures used by the PSAL was reduced to half of that detection limit as the oxide concentration was determined.

\subsubsection{Measurements in Analytical Sequence}

Figure A1 in Appendix A provides plots of the measurements (in analytical sequence) generated by the PSAL for samples prepared using the LM method. Different symbols and colors are used to represent each of the study and standard glasses. Similar plots are provided in Figure A2 in Appendix A for the samples prepared using the PF method. These plots include all of the measurement data from Tables A1 and A2. While obvious patterns in these plots are difficult to find, a pair of $\mathrm{CaO}$ values for one of the study glasses (symbol " $x$ ") does stand out from the other pair of $\mathrm{CaO}$ values (see Figure A1). A similar trend is also observed for a pair of $\mathrm{Li}_{2} \mathrm{O}$ values (see Figure A2). Other significant trends in the analytical process over the course of these measurements are difficult to discern from these plots; more detailed discussions are provided in the following sections.

\subsubsection{Composition Measurements by Glass Identifier}

Figures A3 and A4 in Appendix A provide plots of the oxide concentration measurements by Glass ID (including Batch 1 and $\mathrm{U}_{\text {std }}$ ) by analytical solution ID for the LM and PF preparation methods, respectively. Different symbols and colors are used to represent the different glasses. These plots show the individual measurements across the duplicates of each preparation method and the two ICPAES calibrations within each analytical set. A review of the plots presented in these figures reveals the repeatability of the four individual values for each oxide for each glass. The pair of $\mathrm{CaO}$ values discussed in the previous section (Section 4.1.1) is associated with glass ID SB4VS-02, which corresponds to Lab ID A09LM. There also appears to be a great deal of scatter in the $\mathrm{Fe}_{2} \mathrm{O}_{3}$ and $\mathrm{Li}_{2} \mathrm{O}$ results for the PF preparations of this same glass. In addition, note that the reference value for the $\mathrm{Fe}_{2} \mathrm{O}_{3}$ concentration in the $\mathrm{U}_{\text {std }}$ glass is $13.196 \mathrm{wt} \%$, while the measured values of this oxide are around $10 \mathrm{wt} \%$, and the reference value for $\mathrm{Li}_{2} \mathrm{O}$ for $\mathrm{U}_{\text {std }}$ is $3.057 \mathrm{wt} \%$, while the measured values are around $5 \mathrm{wt} \%$ as shown in Figure A4. Due to these inconsistencies, it was requested that PSAL remeasure the solutions for SB4VS2-02 (Lab ID A09) for both prep methods as well as to re-prep and re-measure the standards (Batch 1 and $U_{\text {std }}$ ) by both prep methods. ${ }^{d, e}$ The results from these

\footnotetext{
${ }^{\mathrm{d}}$ Concerns about values measured for glass ID SB4VS-09 (Lab ID A08) were also expressed by D. Best from PSAL via email on 5/29/2008. Thus, this sample was also re-measured (in addition to SB4VS-02) and the newly measured values were also used in the determination of chemical compositions.

${ }^{\mathrm{e}}$ The $\mathrm{U}_{\text {std }}$ is used for bias-correcting only.
} 
additional measurements are provided in Table A3 in Appendix A and are discussed in the following sections to guide the reader through the method in which these data were re-evaluated and used to support the objectives of this task.

\subsubsection{Batch 1 and Uranium Standard Results}

Figure A5 in Appendix A provides statistical analyses of the Batch 1 and $U_{\text {std }}$ results generated by the LM prep method by calibration block for each oxide of interest (reference values for the oxide concentrations of the standard are given in the header for each set of measurements in the figure). The results include analysis of variance (ANOVA) investigations, which determine statistically significant differences between the means of these groups for each of the oxides for each of the standards. The measured values of $\mathrm{Al}_{2} \mathrm{O}_{3}, \mathrm{MnO}, \mathrm{TiO}_{2}$, and $\mathrm{ZrO}_{2}$ indicate that a significant ICP-AES calibration effect on the block averages at the 5\% significance level for the Batch 1 standard. For the $\mathrm{U}_{\text {std, }} \mathrm{CaO}, \mathrm{CuO}$, and $\mathrm{Na}_{2} \mathrm{O}$ have values that indicate a significant ICP-AES calibration effect on the block averages at the 5\% significance level.

Figure A6 in Appendix A provides a similar set of analyses for the measurements derived from samples prepared via the PF method (reference values for the oxide concentrations of the standard are given in the headers for each set of measurements in the figure). $\mathrm{Li}_{2} \mathrm{O}$ has measurements that indicate significant ICP-AES calibration effects on the block averages at the 5\% significance level for the Batch 1 standard. For the $\mathrm{U}_{\text {std }}$, note that both the $\mathrm{Fe}_{2} \mathrm{O}_{3}$ and $\mathrm{Li}_{2} \mathrm{O}$ measurements of the third block correspond more closely to the reference values than the first two blocks. It is probable the PF results of the $U_{\text {std }}$ standard samples reported in Table A2 were mislabeled. These values are most likely from another glass sample other than the $U_{\text {std. }}$. Therefore, the PF results from Table A2 for $U_{\text {std }}$ will not be utilized in the remainder of the report.

In addition, some of the results from the statistical analyses provide incentive for adjusting the measurements by the effects of the ICP-AES calibration. Therefore, the oxide measurements of the study glasses were bias corrected for the effect of the ICP-AES calibration on each of the analytical blocks and sub-blocks. The average measurement of Batch 1 results for each ICP-AES block/subblock were used to bias correct the $\mathrm{Al}_{2} \mathrm{O}_{3}, \mathrm{~B}_{2} \mathrm{O}_{3}, \mathrm{BaO}, \mathrm{CaO}, \mathrm{Cr}_{2} \mathrm{O}_{3}, \mathrm{CuO}, \mathrm{Fe}_{2} \mathrm{O}_{3}, \mathrm{~K}_{2} \mathrm{O}, \mathrm{Li}_{2} \mathrm{O}, \mathrm{MgO}$, $\mathrm{MnO}, \mathrm{Na}_{2} \mathrm{O}, \mathrm{NiO}, \mathrm{SiO}_{2}$, and $\mathrm{TiO}_{2}$ measurements, and the average measurement of the $\mathrm{U}_{\text {std }}$ values for each set/block were used to bias correct the $\mathrm{U}_{3} \mathrm{O}_{8}$ measurements. Note that this approach utilizes the $\mathrm{LM}$ results for the $\mathrm{U}_{\text {std }}$ and not the PF results. For oxides other than $\mathrm{U}_{3} \mathrm{O}_{8}$, the Batch 1 results were used to conduct the bias correction as long as the reference value for the oxide concentration in the Batch 1 glass was greater than or equal to $0.1 \mathrm{wt} \%$.

The bias correction was conducted as follows. For each oxide, let $\overline{\mathrm{a}}_{\mathrm{ij}}$ be the average measurement for the $\mathrm{i}^{\text {th }}$ oxide at analytical block $\mathrm{j}$ for Batch 1 (or $\mathrm{U}_{\text {std }}$ for uranium), and let $\mathrm{t}_{\mathrm{i}}$ be the reference value for the $\mathrm{i}^{\text {th }}$ oxide for Batch 1 (or for $\mathrm{U}_{\text {std }}$ if uranium). (The averages and reference values are provided in Figures A3 and A4.) Let $\bar{c}_{i j k}$ be the average measurement for the $i^{\text {th }}$ oxide at analytical block $j$ for the $\mathrm{k}^{\text {th }}$ glass. The bias adjustment was conducted as follows

$$
\overline{\mathrm{c}}_{\mathrm{ijk}} \bullet\left(1-\frac{\overline{\mathrm{a}}_{\mathrm{ij}}-\mathrm{t}_{\mathrm{i}}}{\overline{\mathrm{a}}_{\mathrm{ij}}}\right)=\overline{\mathrm{c}}_{\mathrm{ijk}} \bullet \frac{\mathrm{t}_{\mathrm{i}}}{\overline{\mathrm{a}}_{\mathrm{ij}}}
$$

Bias-corrected measurements are indicated by a "bc" suffix, and such adjustments were performed for all of the oxides of this study, except for $\mathrm{Ce}_{2} \mathrm{O}_{3}, \mathrm{La}_{2} \mathrm{O}_{3}, \mathrm{PbO}, \mathrm{SO}_{4}$, and $\mathrm{ZrO}_{2}$. Both measured and 
WSRC-STI-2008-00315

Revision 0

measured "bc" values are included in the discussion that follows. In these discussions bias-corrected values, which are the same as the original values, for $\mathrm{Ce}_{2} \mathrm{O}_{3}, \mathrm{La}_{2} \mathrm{O}_{3}, \mathrm{PbO}, \mathrm{SO}_{4}$, and $\mathrm{ZrO}_{2}$ are included for completeness (e.g., to allow a sum of oxides to be computed for the bias-corrected results).

\subsubsection{Composition Measurements by Glass Identifier with Targeted Compositions}

Figures A7 and A8 in Appendix A provide plots of the oxide concentration measurements by Glass ID (including Batch 1 and $U_{\text {std }}$ ) by Lab ID for the LM and PF preparation methods for each set of analyses. Targeted concentrations of each oxide for each of the study glasses and reference values for the standards are also provided as part of these figures. In addition, the auxiliary measurements of Table A4 are also included in these plots, which show the individual measurements across the duplicates of each preparation method and the replicate ICP-AES calibrations.

A review of the plots reveals the repeatability of the individual oxide values for each glass and serves as a basis for discerning questionable measurement values (and their possible causes). In the plots for $\mathrm{CaO}$, there are obvious inconsistencies in one glass (SB4VS2-02). This is one of the glasses for which re-measurements by PSAL were requested. As seen in the $\mathrm{CaO}$ plots, the re-measured values of the solutions are quite similar to the original measurements suggesting that the sample preparation is likely the cause of the differences. The target concentration for $\mathrm{CaO}$ for this glass was $1.042 \mathrm{wt} \%$; the second LM preparation yielded values near this target, while the first LM preparation yieled values of approximately $1.5 \mathrm{wt} \%$. A review of these results led the authors to conclude that the values near $\sim 1.5 \mathrm{wt} \%$ were not representative of the compositions of the glass. Thus, these values were excluded from further consideration for this report. These questionable data from SB4VS2-02 are listed in Table 2 along with the $\mathrm{U}_{\text {std }}$ measurements from the original PF preparations that were highlighted in Section 4.1.3. The values in this table were excluded from further analysis and were not used in the determination of the chemical compositions of the study glasses or standards.

\subsubsection{Measured versus Targeted Compositions}

The remaining measurements for each oxide for each glass (i.e., all of the measurements in Tables A1 through A3 excluding the values appearing in Table 2) were averaged to determine a representative chemical composition for each glass. These determinations were conducted for both the measured and bias-corrected data. A sum of oxides was also computed for each glass based upon both the measured and bias-corrected values. Figure A9 in Appendix A provides plots showing results of each oxide for each glass in order to highlight the comparisons among the measured, bias-corrected, and targeted values. In general, after the elimination of the questionable values, the measured values do agree with the target compositions. The $\mathrm{SiO}_{2}$ value for $\mathrm{SB} 4 \mathrm{VS} 2-02$ is somewhat higher than the targeted concentration for this oxide for this glass.

Table A4 in Appendix A provides a summary of the average compositions as well as the targeted compositions. Also included in the table are relative differences between the measured or biascorrected values and the targeted values, which are shaded when they are greater than or equal to $5 \%$. Notice that the targeted sums of oxides for the standard glasses do not sum to $100 \%$ due to an incomplete coverage of the oxides in the Batch 1 and $U_{\text {std }}$ glasses. All of the sums of oxides (both measured and bias-corrected) for the study glasses fall within the interval of 95 to $105 \mathrm{wt} \%$. Overall, these comparisons between the measured and targeted compositions suggest only minor difficulties in hitting the targeted compositions for some of the oxides for some of the glasses, none of which should affect the outcome of the variability study. 
WSRC-STI-2008-00315

Revision 0

Table 2. Questionable Measurements Eliminated From Further Analysis

\begin{tabular}{|c|c|c|c|c|c|c|}
\hline Study Glass \# & Glass ID & Lab ID & Oxide & Measured & Measured bc & Targeted \\
\hline 2 & SB4VS2-02 & A09 11 & $\mathrm{CaO}\left(\mathrm{wt}^{\circ} \%\right)$ & 1.50 & 1.61 & 1.04 \\
\hline 2 & SB4VS2-02 & A09 12 & $\mathrm{CaO}(\mathrm{wt} \%)$ & 1.50 & 1.61 & 1.04 \\
\hline 2 & SB4VS2-02 & A09LM11 & $\mathrm{CaO}(\mathrm{wt} \%)$ & 1.55 & 1.61 & 1.04 \\
\hline 2 & SB4VS2-02 & A09LM12 & $\mathrm{CaO}(\mathrm{wt} \%)$ & 1.54 & 1.63 & 1.04 \\
\hline 2 & SB4VS2-02 & A09PF11 & $\mathrm{B}_{2} \mathrm{O}_{3}(\mathrm{wt} \%)$ & 9.02 & 9.21 & 9.10 \\
\hline 2 & SB4VS2-02 & A09PF11 & $\mathrm{Fe}_{2} \mathrm{O}_{3}(\mathrm{wt} \%)$ & 12.57 & 12.52 & 10.79 \\
\hline 2 & SB4VS2-02 & A09PF11 & $\mathrm{Li}_{2} \mathrm{O}(\mathrm{wt} \%)$ & 3.08 & 3.16 & 5.20 \\
\hline 2 & SB4VS2-02 & A09PF12 & $\mathrm{B}_{2} \mathrm{O}_{3}(\mathrm{wt} \%)$ & 8.76 & 8.99 & 9.10 \\
\hline 2 & SB4VS2-02 & A09PF12 & $\mathrm{Fe}_{2} \mathrm{O}_{3}(\mathrm{wt} \%)$ & 11.87 & 11.99 & 10.79 \\
\hline 2 & SB4VS2-02 & A09PF12 & $\mathrm{Li}_{2} \mathrm{O}(\mathrm{wt} \%)$ & 3.01 & 3.14 & 5.20 \\
\hline 200 & $\mathrm{U}_{\mathrm{std}}$ & $\mathrm{U}_{\text {std }} \mathrm{PF} 11$ & $\mathrm{~B}_{2} \mathrm{O}_{3}(\mathrm{wt} \%)$ & 9.11 & 9.31 & 9.21 \\
\hline 200 & $\mathrm{U}_{\text {std }}$ & $\mathrm{U}_{\text {std }} \mathrm{PF} 11$ & $\mathrm{Fe}_{2} \mathrm{O}_{3}(\mathrm{wt} \%)$ & 10.47 & 10.42 & 13.20 \\
\hline 200 & $\mathrm{U}_{\text {std }}$ & $\mathrm{U}_{\text {std }} \mathrm{PF} 11$ & $\mathrm{Li}_{2} \mathrm{O}(\mathrm{wt} \%)$ & 5.17 & 5.30 & 3.06 \\
\hline 200 & $\mathrm{U}_{\text {std }}$ & $\mathrm{U}_{\text {std }} \mathrm{PF} 12$ & $\mathrm{~B}_{2} \mathrm{O}_{3}(\mathrm{wt} \%)$ & 8.66 & 8.85 & 9.21 \\
\hline 200 & $\mathrm{U}_{\text {std }}$ & $\mathrm{U}_{\text {std }} \mathrm{PF} 12$ & $\mathrm{Fe}_{2} \mathrm{O}_{3}(\mathrm{wt} \%)$ & 10.11 & 10.07 & 13.20 \\
\hline 200 & $\mathrm{U}_{\text {std }}$ & $\mathrm{U}_{\text {std }} \mathrm{PF} 12$ & $\mathrm{Li}_{2} \mathrm{O}(\mathrm{wt} \%)$ & 5.12 & 5.25 & 3.06 \\
\hline 200 & $\mathrm{U}_{\text {std }}$ & $\mathrm{U}_{\text {std }} \mathrm{PF} 13$ & $\mathrm{~B}_{2} \mathrm{O}_{3}(\mathrm{wt} \%)$ & 8.63 & 8.82 & 9.21 \\
\hline 200 & $\mathrm{U}_{\text {std }}$ & $\mathrm{U}_{\text {std }} \mathrm{PF} 13$ & $\mathrm{Fe}_{2} \mathrm{O}_{3}(\mathrm{wt} \%)$ & 9.98 & 9.94 & 13.20 \\
\hline 200 & $\mathrm{U}_{\text {std }}$ & $\mathrm{U}_{\mathrm{std}} \mathrm{PF} 13$ & $\mathrm{Li}_{2} \mathrm{O}(\mathrm{wt} \%)$ & 5.10 & 5.23 & 3.06 \\
\hline 200 & $\mathrm{U}_{\text {std }}$ & $\mathrm{U}_{\text {std }} \mathrm{PF} 21$ & $\mathrm{~B}_{2} \mathrm{O}_{3}(\mathrm{wt} \%)$ & 9.02 & 9.25 & 9.21 \\
\hline 200 & $\mathrm{U}_{\text {std }}$ & $\mathrm{U}_{\text {std }} \mathrm{PF} 21$ & $\mathrm{Fe}_{2} \mathrm{O}_{3}(\mathrm{wt} \%)$ & 10.27 & 10.37 & 13.20 \\
\hline 200 & $\mathrm{U}_{\text {std }}$ & $\mathrm{U}_{\text {std }} \mathrm{PF} 21$ & $\mathrm{Li}_{2} \mathrm{O}(\mathrm{wt} \%)$ & 5.15 & 5.36 & 3.06 \\
\hline 200 & $\mathrm{U}_{\text {std }}$ & $\mathrm{U}_{\text {std }} \mathrm{PF} 22$ & $\mathrm{~B}_{2} \mathrm{O}_{3}(\mathrm{wt} \%)$ & 8.76 & 8.99 & 9.21 \\
\hline 200 & $\mathrm{U}_{\text {std }}$ & $\mathrm{U}_{\text {std }} \mathrm{PF} 22$ & $\mathrm{Fe}_{2} \mathrm{O}_{3}(\mathrm{wt} \%)$ & 10.09 & 10.20 & 13.20 \\
\hline 200 & $\mathrm{U}_{\text {std }}$ & $\mathrm{U}_{\text {std }} \mathrm{PF} 22$ & $\mathrm{Li}_{2} \mathrm{O}(\mathrm{wt} \%)$ & 5.10 & 5.31 & 3.06 \\
\hline 200 & $\mathrm{U}_{\text {std }}$ & $\mathrm{U}_{\text {std }} \mathrm{PF} 23$ & $\mathrm{~B}_{2} \mathrm{O}_{3}(\mathrm{wt} \%)$ & 8.69 & 8.92 & 9.21 \\
\hline 200 & $\mathrm{U}_{\text {std }}$ & $\mathrm{U}_{\text {std }} \mathrm{PF} 23$ & $\mathrm{Fe}_{2} \mathrm{O}_{3}(\mathrm{wt} \%)$ & 10.05 & 10.16 & 13.20 \\
\hline 200 & $\mathrm{U}_{\mathrm{std}}$ & $\mathrm{U}_{\text {std }} \mathrm{PF} 23$ & $\mathrm{Li}_{2} \mathrm{O}(\mathrm{wt} \%)$ & 5.10 & 5.31 & 3.06 \\
\hline
\end{tabular}

\subsubsection{MAR Assessment}

Another assessment that can be made for the SB4 variability study (VS) glasses is how well they satisfy the MAR criteria of DWPF's Product Composition Control System (PCCS). The results of this assessment are shown in Table 3. The columns in the table give the percent waste loading ( $\% \mathrm{WL}$ ), the frit, the glass identifier with compositional view, the B del $\mathrm{Gp}$ ( $\Delta \mathrm{Gp}$ value for boron), the predicted normalized leachate for boron in grams/Liter $(\mathrm{NL}[\mathrm{B}(\mathrm{g} / \mathrm{L})])$, the liquidus temperature prediction in degrees Celsius $\left(\mathrm{T}_{\mathrm{L}}\right.$ Pred $\left({ }^{\circ} \mathrm{C}\right)$ ), the viscosity prediction at $1150^{\circ} \mathrm{C}$ in Poise (Visc Pred (P)), the sum of oxides (in wt $\%$ ), the nepheline discriminator value, and the overall MAR assessment.

All of the glasses that were selected for this study satisfy these criteria and are deemed processable and acceptable for the DWPF, except for the SB4VS2-03 (sludge-only at 38\% WL) target composition. This glass fails the $\mathrm{T}_{\mathrm{L}}$ criterion and would not be considered processable based on SME acceptability decisions. The relatively high $\mathrm{T}_{\mathrm{L}}$ prediction is due to the low $\mathrm{Na}_{2} \mathrm{O}$ content of the sludge-only flowsheet and high WL target. 
Table 3. Results of the MAR Assessment of SB4 VS Glasses for Measured, Bias Corrected and Targeted Compositional Views

\begin{tabular}{|c|c|c|c|c|c|c|c|c|}
\hline$\% \mathrm{WL}$ & Sample ID/Frit & Sludge Type & $\begin{array}{c}\text { B Del Gp } \\
\text { Value }\end{array}$ & $\begin{array}{c}\mathrm{NL} \\
{[\mathrm{B}(\mathrm{g} / \mathrm{L})]} \\
\end{array}$ & $\begin{array}{c}\text { TL Pred } \\
\left({ }^{\circ} \mathrm{C}\right) \\
\end{array}$ & $\begin{array}{c}\text { Visc } \\
\text { Pred }(\mathrm{P}) \\
\end{array}$ & $\begin{array}{l}\text { Neph } \\
\text { Value } \\
\end{array}$ & MAR Status \\
\hline 32 & SB4VS2-01/Frit 510 & Tk 40 Post Late Decant (5-6-08)/targeted & -8.33 & 0.40 & 942.4 & 51.5 & 0.720 & \\
\hline 35 & SB4VS2-02/Frit 510 & Tk 40 Post Late Decant (5-6-08)/targeted & -8.11 & 0.37 & 981.6 & 48.3 & 0.701 & \\
\hline 38 & SB4VS2-03/Frit 510 & Tk 40 Post Late Decant (5-6-08)/targeted & -7.89 & 0.34 & 1018.0 & 45.1 & 0.681 & $\mathrm{TL}$ \\
\hline 32 & SB4VS2-04/Frit 510 & Tk 40 Post Late Decant w ARP (5-6-08)/targeted & -8.71 & 0.47 & 925.5 & 47.7 & 0.720 & \\
\hline 35 & SB4VS2-05/Frit 510 & Tk 40 Post Late Decant w ARP (5-6-08)/targeted & -8.52 & 0.44 & 963.5 & 44.2 & 0.700 & \\
\hline 38 & SB4VS2-06/Frit 510 & Tk 40 Post Late Decant w ARP (5-6-08)/targeted & -8.34 & 0.41 & 998.6 & 40.7 & 0.680 & \\
\hline 32 & SB4VS2-07/Frit 510 & Tk 40 Post Late Decant w ARP $+2 \%$ Na2O (5-6-08)/targeted & -9.33 & 0.61 & 903.6 & 42.9 & 0.715 & \\
\hline 35 & SB4VS2-08/Frit 510 & Tk 40 Post Late Decant w ARP $+2 \%$ Na2O (5-6-08)/targeted & -9.20 & 0.58 & 940.5 & 39.1 & 0.695 & \\
\hline 38 & SB4VS2-09/Frit 510 & Tk 40 Post Late Decant w ARP $+2 \% \mathrm{Na} 2 \mathrm{O}(5-6-08) /$ targeted & -9.07 & 0.55 & 974.5 & 35.5 & 0.674 & \\
\hline 32 & SB4VS2-01/Frit 510 & Tk 40 Post Late Decant (5-6-08)/measured & -8.18 & 0.38 & 945.4 & 52.7 & 0.720 & \\
\hline 35 & SB4VS2-02/Frit 510 & Tk 40 Post Late Decant (5-6-08)/measured & -8.17 & 0.38 & 964.6 & 54.1 & 0.697 & \\
\hline 38 & SB4VS2-03/Frit 510 & Tk 40 Post Late Decant (5-6-08)/measured & -7.80 & 0.33 & 1004.8 & 46.1 & 0.680 & \\
\hline 32 & SB4VS2-04/Frit 510 & Tk 40 Post Late Decant w ARP (5-6-08)/measured & -8.63 & 0.46 & 920.3 & 49.4 & 0.717 & \\
\hline 35 & SB4VS2-05/Frit 510 & Tk 40 Post Late Decant w ARP (5-6-08)/measured & -8.47 & 0.43 & 961.7 & 45.0 & 0.699 & \\
\hline 38 & SB4VS2-06/Frit 510 & Tk 40 Post Late Decant w ARP (5-6-08)/measured & -8.14 & 0.38 & 987.0 & 46.7 & 0.680 & \\
\hline 32 & SB4VS2-07/Frit 510 & Tk 40 Post Late Decant w ARP $+2 \% \mathrm{Na} 2 \mathrm{O}(5-6-08) /$ measured & -9.12 & 0.56 & 904.4 & 45.1 & 0.713 & \\
\hline 35 & SB4VS2-08/Frit 510 & Tk 40 Post Late Decant w ARP $+2 \% \mathrm{Na} 2 \mathrm{O}(5-6-08) /$ measured & -9.10 & 0.56 & 928.8 & 43.5 & 0.693 & \\
\hline 38 & SB4VS2-09/Frit 510 & Tk 40 Post Late Decant w ARP $+2 \% \mathrm{Na} 2 \mathrm{O}(5-6-08) /$ measured & -9.07 & 0.55 & 956.1 & 40.6 & 0.672 & \\
\hline 32 & SB4VS2-01/Frit 510 & Tk 40 Post Late Decant (5-6-08)/measured bc & -8.11 & 0.37 & 951.6 & 53.8 & 0.722 & \\
\hline 35 & SB4VS2-02/Frit 510 & Tk 40 Post Late Decant (5-6-08)/measured bc & -8.05 & 0.36 & 973.4 & 58.0 & 0.702 & \\
\hline 38 & SB4VS2-03/Frit 510 & Tk 40 Post Late Decant (5-6-08)/measured bc & -7.70 & 0.31 & 1011.6 & 47.7 & 0.681 & \\
\hline 32 & SB4VS2-04/Frit 510 & Tk 40 Post Late Decant w ARP (5-6-08)/measured bc & -8.54 & 0.44 & 927.0 & 50.5 & 0.719 & \\
\hline 35 & SB4VS2-05/Frit 510 & Tk 40 Post Late Decant w ARP (5-6-08)/measured bc & -8.37 & 0.41 & 968.6 & 46.2 & 0.700 & \\
\hline 38 & SB4VS2-06/Frit 510 & Tk 40 Post Late Decant w ARP (5-6-08)/measured bc & -8.02 & 0.36 & 994.3 & 48.4 & 0.682 & \\
\hline 32 & SB4VS2-07/Frit 510 & Tk 40 Post Late Decant $w$ ARP $+2 \% \mathrm{Na} 2 \mathrm{O}(5-6-08) /$ measured bc & -9.02 & 0.54 & 911.3 & 46.3 & 0.715 & \\
\hline 35 & SB4VS2-08/Frit 510 & Tk 40 Post Late Decant w ARP $+2 \% \mathrm{Na} 2 \mathrm{O}(5-6-08) /$ measured bc & -8.98 & 0.53 & 936.5 & 44.9 & 0.695 & \\
\hline 38 & SB4VS2-09/Frit 510 & Tk 40 Post Late Decant w ARP $+2 \% \mathrm{Na} 2 \mathrm{O}(5-6-08) /$ measured bc & -8.95 & 0.52 & 965.3 & 43.7 & 0.677 & \\
\hline
\end{tabular}


WSRC-STI-2008-00315

Revision 0

\subsection{Crystallization}

\subsubsection{Visual Observations}

Prior to discussing the visual observations, a brief explanation of the terms used to describe the asfabricated (quenched) and ccc glasses is necessary. "Surface" refers to the top of the sample that has not touched the steel plate during quenching or the walls of the crucible during the ccc treatment. The term "bulk" refers to the cross-section of the glass sample. "Homogeneous" indicates that there is no crystallization evident on the surface or in the bulk of the glass. Other terms such as "haze", "clusters", and/or "silver/metallic patches" imply that the surface or bulk of the glass contains crystals or some other characteristic feature. "Black and shiny" implies that crystallization is not apparent to the un-aided eye.

The surface and bulk of each as-fabricated (quenched) glass, except SB4VS2-03 (sludge-only at 38\% WL), were "black and shiny," which indicates that the glasses are free of crystallization and homogeneous. As previously noted, SB4VS2-03 was predicted to have a higher $\mathrm{T}_{\mathrm{L}}$ due to the lower $\mathrm{Na}_{2} \mathrm{O}$ content of the glass. A summary of the visual observations is given in Table 4. The SB4VS203 sample did contain a slight amount of crystallization on the surface (i.e. silver swirls); however, the bulk was clean.

Crystallization was much more prevalent in the ccc glasses given that the kinetics for crystallization are more favorable during the slow cooling of the ccc treatment. None of the ccc glasses were determined to be homogeneous based on visual observations as shown in Table 4. The ccc glasses did contain some degree of crystallization on the surface, characterized by "haze" and "patches of silver crystals." Historically, metallic-like features on the surface of DWPF glasses are due to the precipitation of spinels during the slower cooling process. Crystallization was not present in the bulk of any glasses.

\subsubsection{XRD}

Each of the quenched glasses was amorphous (within the detection limit of the instrument), which, in general, corresponded to the visual observations (Section 4.2.1). Only one of the ccc samples contain crystalline material, while all others were amorphous (within the detection limit of the instrument). A cross between trevorite and magnetite was detected in sample SB4VS2-03ccc (sludge-only at 38\% WL). These results were not surprising as crystals were already present on the surface of the quenched sample. A representative pattern ${ }^{\mathrm{f}}$ of an amorphous sample is shown in Figure 1, while the XRD pattern of SB4VS2-03ccc is shown in Figure 2.

A majority of the samples did contain visual evidence of surface crystals, but crystallization was not observed in any of the XRD patterns (except SB4VS2-03ccc). Since the surface is only a small fraction of the sample and sampling for XRD is random, it is probable that the crystalline content was much below the detection limit of the instrument.

\footnotetext{
${ }^{\mathrm{f}}$ The remainder of the XRD patterns (quenched and ccc) can be viewed in the laboratory notebook for the SB4 variability study on pages 151-152 (WSRC-NB-2006-00168).
} 
Table 4. Visual Observations of Quenched and CCC Glasses

\begin{tabular}{|c|c|c|c|c|c|}
\hline \multirow{2}{*}{ Glass ID } & \multirow{2}{*}{ Frit } & \multirow{2}{*}{ WL } & \multicolumn{2}{|c|}{ Visual Observations of Quenched Glasses } & \multirow{2}{*}{ XRD } \\
\hline & & & Surface & Bulk & \\
\hline SB4VS2-01 & \multirow{9}{*}{510} & 32 & Black and shiny & Clean & Amorphous \\
\hline SB4VS2-02 & & 35 & Black and shiny & Clean & Amorphous \\
\hline SB4VS2-03 & & 38 & Small amount of silver swirls & Clean & Amorphous \\
\hline SB4VS2-04 & & 32 & Black and shiny & Clean & Amorphous \\
\hline SB4VS2-05 & & 35 & Black and shiny & Clean & Amorphous \\
\hline SB4VS2-06 & & 38 & Black and shiny & Clean & Amorphous \\
\hline SB4VS2-07 & & 32 & Black and shiny & Clean & Amorphous \\
\hline SB4VS2-08 & & 35 & Black and shiny & Clean & Amorphous \\
\hline SB4VS2-09 & & 38 & Black and shiny & Clean & Amorphous \\
\hline
\end{tabular}

\begin{tabular}{|c|c|c|c|c|c|}
\hline \multirow{2}{*}{ Glass ID } & \multirow{2}{*}{ Frit } & \multirow{2}{*}{ WL } & \multicolumn{2}{|c|}{ Visual Observations of CCC Glasses } & \multirow{2}{*}{ XRD } \\
\hline & & & Surface & Bulk & \\
\hline SB4VS2-01 & \multirow{9}{*}{510} & 32 & Light haze with some crystals & Clean & Amorphous \\
\hline SB4VS2-02 & & 35 & Light haze with some patches of silver crystals & Clean & Amorphous \\
\hline SB4VS2-03 & & 38 & Light haze with some patches of silver crystals & Clean & Magnetite/Trevorite \\
\hline SB4VS2-04 & & 32 & Light haze with a few scattered silver crystals & Clean & Amorphous \\
\hline SB4VS2-05 & & 35 & Slight haze with a few patches of bright silver crystals & Clean & Amorphous \\
\hline SB4VS2-06 & & 38 & Slight haze with a few patches of bright silver crystals & Clean & Amorphous \\
\hline SB4VS2-07 & & 32 & Very light haze & Clean & Amorphous \\
\hline SB4VS2-08 & & 35 & Light haze with a few patches of silver crystals & Clean & Amorphous \\
\hline SB4VS2-09 & & 38 & Light haze with a few scattered patches of silver crystals & Clean & Amorphous \\
\hline
\end{tabular}




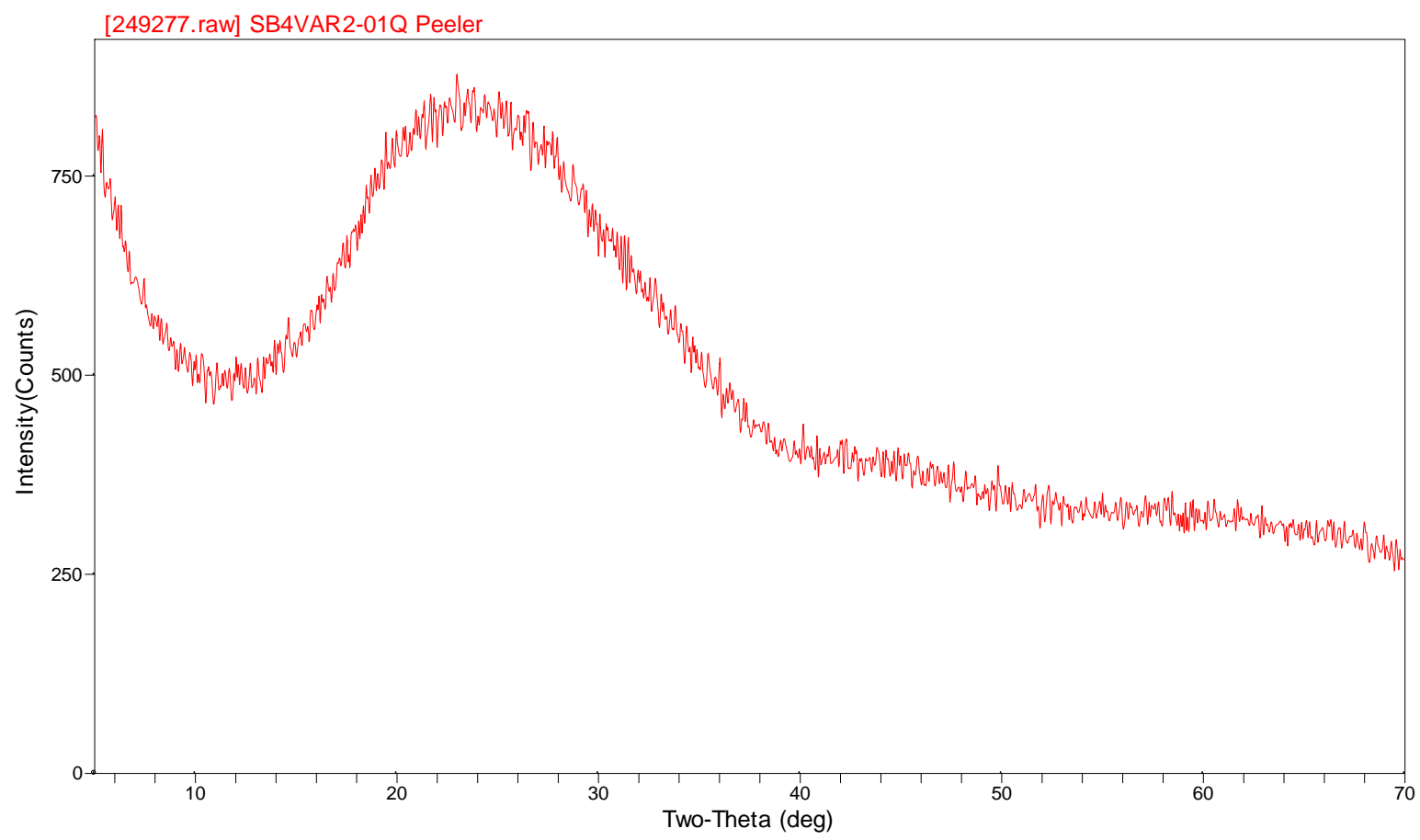

Figure 1. A representative XRD pattern of an amorphous sample. Note: "SB4VAR2" on sample label (upper left hand corner) should be "SB4VS2."

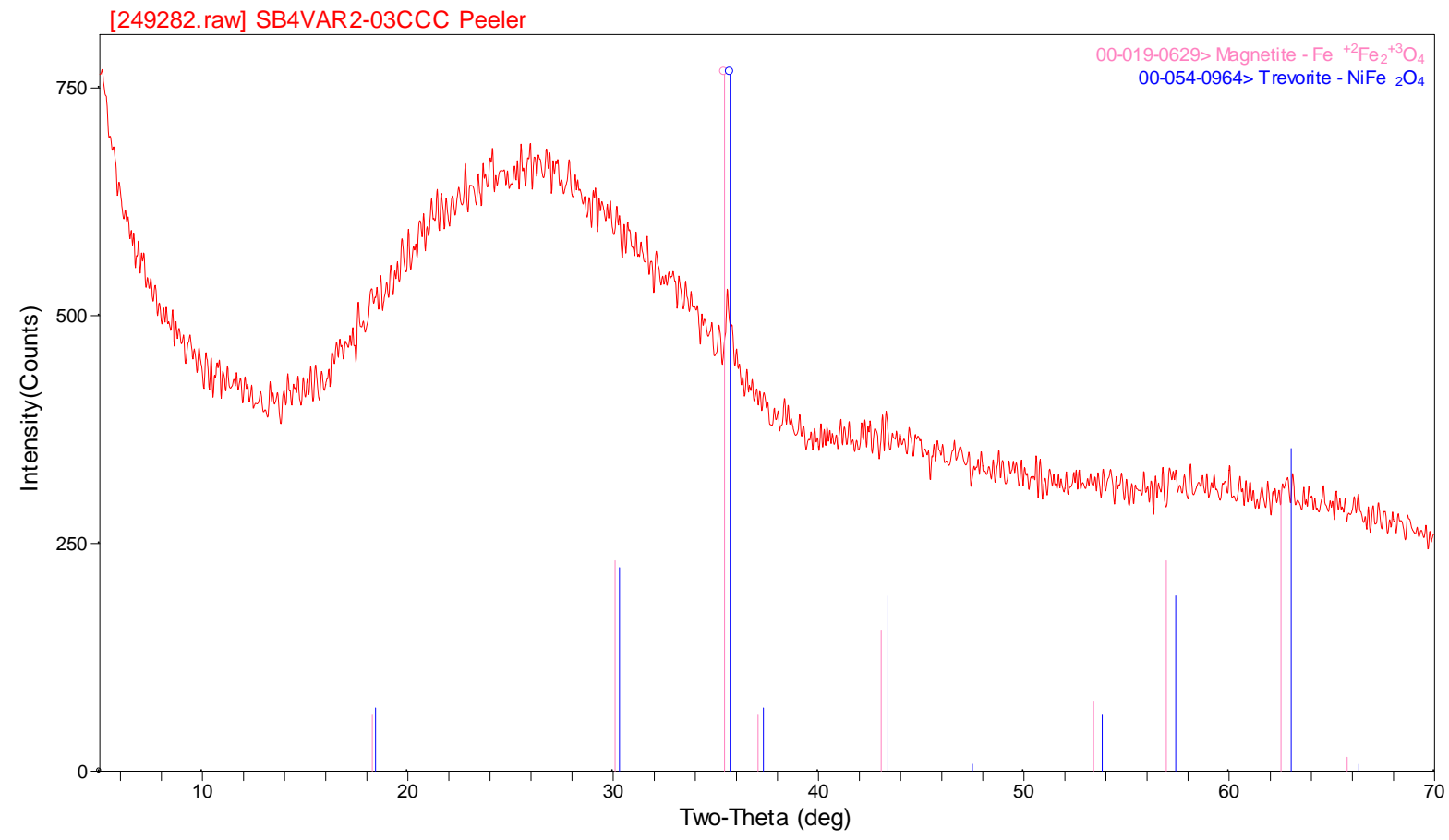

Figure 2. XRD pattern of the ccc version of the SB4VS2-03 glass (sludge-only at 38\% WL). Note: “SB4VAR2" on sample label (upper left hand corner) should be "SB4VS2." 


\subsection{Statistical Review of the PCT Results}

Table B1 in Appendix B provides the elemental leachate concentration measurements determined by the PSAL for the solution samples generated by the PCTs. Any measurement in Table B1 below the detection limit of the analytical procedure (indicated by a " $<$ ") was replaced by $1 / 2$ of the detection limit in subsequent analyses. The measured solution-weight loss over the course of the 7-day test does not indicate a solution-weight loss problem for any of the samples.

In addition to adjustments for detection limits, the values were adjusted for the dilution factors: the values for the study glasses, the blanks, and the ARM glass in Table B1 were multiplied by 1.6667 to determine the values in parts per million (ppm) and the values for EA were multiplied by 16.6667. Table B2 in Appendix B provides the resulting measurements.

\subsubsection{Measurements in Analytical Sequence}

Figure B1 in Appendix B provides plots of the leachate ( $\mathrm{ppm}$ ) concentrations in analytical sequence as generated by the PSAL for all of the data and for the data from only the study glasses, respectively. A different color and symbol are used for each study glass or standard. No issues are seen in these plots.

\subsubsection{Results for the Samples of the Multi-Element Solution Standard}

Figure B2 in Appendix B provides analyses of the PSAL measurements of the samples of the multielement solution standard by analytical set and ICP-AES calibration block. An ANOVA was used to determine any statistically significant differences among the block averages for these samples for each element of interest and is included in these figures. There was no indication of a statistically significant difference (at a 5\% level) among the averages of these measurements for any of the elements of interest except for Li. However, averaging the ppm values for each set of triplicates helps to minimize the impact of any potential instrumentation effects.

Table 5 summarizes the average measurements and the reference values for the four primary elements of interest (B, Li, $\mathrm{Na}$ and $\mathrm{Si}$ ). The results indicate consistent and accurate measurements from the PSAL processes used to conduct these analyses.

Table 5. Results from Samples of the Multi-Element Solution Standard

\begin{tabular}{|c|c|c|c|c|}
\hline Analytical & Avg B & Avg Li & Avg Na & Avg Si \\
\hline Block & (ppm) & $\mathbf{( p p m ) ~}$ & $\mathbf{( p p m )}$ & $\mathbf{( p p m )}$ \\
\hline 1 & 20.1 & 9.8 & 81.4 & 49.8 \\
\hline 2 & 19.4 & 9.5 & 77.8 & 49.3 \\
\hline 3 & 19.0 & 9.5 & 80.9 & 48.1 \\
\hline Grand Average & 19.5 & 9.6 & 80.1 & 49.1 \\
\hline Reference Value & 20 & 10 & 81 & 50 \\
\hline \% difference & $-2.61 \%$ & $-3.79 \%$ & $-1.17 \%$ & $-1.89 \%$ \\
\hline
\end{tabular}


WSRC-STI-2008-00315

Revision 0

\subsubsection{Measurements by Glass Identifier}

Figure B3 in Appendix B provide plots of the leachate concentrations for each type of submitted sample: the study glasses by heat treatment (quenched and ccc) and the standards (EA, ARM, the multi-element solution standard, and blanks). Figure B4 in Appendix B provide plots of the leachate concentrations for the PCT results of just the study glasses by heat treatment. These plots suggest some scatter in the triplicate values for some analytes for some of the glasses. Also, note the small differences between the quenched and ccc values for each of the study glasses.

\subsubsection{Normalized PCT Results}

PCT leachate concentrations are typically normalized using the cation composition (expressed as a weight percent) in the glass to obtain a grams-per-liter $(\mathrm{g} / \mathrm{L})$ leachate concentration. The normalization of the PCTs is usually conducted using the measured compositions of the glasses. This method is the preferred normalization process for the PCTs. For completeness, the targeted cation and the bias-corrected cation compositions were also used to conduct this normalization.

The common logarithm of the normalized PCT (normalized leachate, NL) for each element of interest was determined and used for comparison. To accomplish this computation, one must

1.Determine the common logarithm of the elemental ppm leachate concentration for each of the triplicates and each of the elements of interest (these values are provided in Table B2 of Appendix B),

2.Average the common logarithms over the triplicates for each element of interest, and then

Normalizing Using Measured Composition (preferred method)

3. Subtract a quantity equal to 1 plus the common logarithm of the average cation measured concentration (expressed as a weight percent of the glass) from the average computed in step 2 .

Or Normalizing Using Target Composition

3. Subtract a quantity equal to 1 plus the common logarithm of the target cation concentration (expressed as a weight percent of the glass) from the average computed in step 2 .

Or Normalizing Using Measured Bias-Corrected Composition

3. Subtract a quantity equal to 1 plus the common logarithm of the measured biascorrected cation concentration (expressed as a weight percent of the glass) from the average computed in step 2.

Figure B5 in Appendix B provides scatter plots for these results and offers an opportunity to investigate the consistency in the leaching across the elements for the glasses of this study. All combinations of the normalizations of the PCTs (i.e., those generated using the targeted, measured, and bias-corrected compositional views) and both heat treatments are represented in the series of scatter plots. Consistency in the leaching across the elements is typically demonstrated by a high degree of linear correlation among the values for pairs of these elements, which is true for this study as the smallest correlation in this plot is that for $\mathrm{Na}$ and $\mathrm{Si}$, with a value of $\sim 97 \%$. 
WSRC-STI-2008-00315

Revision 0

Table 6 summarizes the normalized PCTs for the glasses of this study, which are listed by glass ID.

\subsubsection{Acceptability of the Variability Study Glasses}

All of the variability study glasses are acceptable relative to the benchmark EA glass as shown in Table 6. The NL[B] values of the study glasses range from $0.636 \mathrm{~g} / \mathrm{L}$ to $0.766 \mathrm{~g} / \mathrm{L}$ regardless of thermal history, compositional view, sludge composition or WL. These NL [B] values are more than an order of magnitude less than the $16.695 \mathrm{~g} / \mathrm{L}$ reported for EA.

\subsubsection{Effects of Heat Treatment on PCTs}

Figure B6 in Appendix B provides a series of plots and statistical comparisons that demonstrate the effects of heat treatment on the common logarithm ppm-responses of interest of the triplicate PCTs for each element for each study glass. The quenched version of a given glass yielded measurements indicating a significantly different mean $\log (\mathrm{ppm})$ response (at the $5 \%$ significance level) as compared to the ccc version of the glass for a given element if the Prob $>|\mathbf{t}|$ value in the figure is 0.05 or smaller. Only Na value of one glass (SB4VS2-04) showed a statistically significant difference (at the $5 \%$ level) in the means of the two heat treatments. In addition, in this instance, the mean $\log [\mathrm{Na}$ (ppm)] was larger for the quenched heat treatment than the mean $\log [\mathrm{Na}(\mathrm{ppm})]$ for the ccc version of this glass. These results suggest that there are no indications of these glasses being sensitive to heat treatment.

Figure B7 in Appendix B provides a series of plots that demonstrate the effects of heat treatment on the normalized PCT response based on the three different compositional views: measured, measured bias-corrected, and targeted. The plots are grouped by the sludge type used to determine the target composition for the glass. Waste loadings are also shown as part of the information on these plots. These results indicate that there is very little statistical difference in PCT responses as a function of heat treatment. There is some evidence of a trend toward a less durable glass as WL increases for some of the sludge projections.

\subsubsection{Predicted versus Measured PCTs}

Figure B8 in Appendix B provides plots of the DWPF models that relate the logarithm of the normalized PCT (for each element of interest) to a linear function of a free energy of hydration term $\left(\Delta \mathrm{G}_{\mathrm{p}}, \mathrm{kcal} / 100 \mathrm{~g}\right.$ glass$)$ derived from all of the glass compositional views and heat treatments. ${ }^{9}$ Prediction limits (at a 95\% confidence) for an individual PCT result are also plotted along with the linear fit. The EA and ARM results are also indicated on these plots. Figure B9 in Appendix B provides a version of these plots for the quenched glasses only while Figure B10 in Appendix B provides a version for ccc glasses only. Not only are the study glasses acceptable relative to the EA glass, but the plots illustrate the predictablility of the glasses by the current $\Delta \mathrm{G}_{\mathrm{p}}$ models. Figure 3 provides a close look at the PCT response for boron. All of the study glasses lie within the 95\% confidence intervals of the model predictions. 
Table 6. Normalized PCTs by Glass ID/Compositional View for SB4/Second Decant Glasses with Frit 510

\begin{tabular}{|c|c|c|c|c|c|c|c|c|c|c|c|c|c|}
\hline Glass & WL $\%$ & Sludge Case & $\begin{array}{c}\text { Heat } \\
\text { Treatment }\end{array}$ & $\begin{array}{l}\text { Compositional } \\
\text { View }\end{array}$ & $\begin{array}{l}\text { Nepheline } \\
\text { Assessment }\end{array}$ & $\begin{array}{c}\log \\
\mathrm{NL}[\mathrm{B} \\
(\mathrm{g} / \mathrm{L})] \\
\end{array}$ & $\begin{array}{c}\log \\
N L[L i \\
(\mathrm{g} / \mathrm{L})] \\
\end{array}$ & $\begin{array}{c}\log \\
\mathrm{NL}[\mathrm{Na} \\
(\mathrm{g} / \mathrm{L})] \\
\end{array}$ & $\begin{array}{c}\log \mathrm{NL} \\
{[\mathrm{Si}(\mathrm{g} / \mathrm{L})]}\end{array}$ & $\begin{array}{c}\mathrm{NL}[\mathrm{B} \\
(\mathrm{g} / \mathrm{L})]\end{array}$ & $\begin{array}{l}\mathrm{NL}[\mathrm{Li} \\
(\mathrm{g} / \mathrm{L})]\end{array}$ & $\begin{array}{c}\mathrm{NL}[\mathrm{Na} \\
(\mathrm{g} / \mathrm{L})]\end{array}$ & $\begin{array}{l}\mathrm{NL}[\mathrm{Si} \\
(\mathrm{g} / \mathrm{L})]\end{array}$ \\
\hline ARM & . & & ref & reference & 0.753 & -0.3163 & -0.2344 & -0.2966 & -0.5561 & 0.483 & 0.583 & 0.505 & 0.278 \\
\hline EA & . & & ref & reference & 0.704 & 1.2055 & 0.9439 & 1.1009 & 0.5705 & 16.052 & 8.788 & 12.614 & 3.719 \\
\hline SB4VS2-01 & 32 & $\begin{array}{c}\text { Tk } 40 \text { Post Late Decant } \\
(5-6-08)\end{array}$ & $\mathrm{ccc}$ & targeted & 0.720 & -0.1859 & -0.1487 & -0.2454 & -0.3453 & 0.652 & 0.710 & 0.568 & 0.452 \\
\hline SB4VS2-02 & 35 & $\begin{array}{c}\text { Tk } 40 \text { Post Late Decant } \\
(5-6-08)\end{array}$ & $\mathrm{ccc}$ & targeted & 0.701 & -0.1577 & -0.1264 & -0.2072 & -0.3397 & 0.696 & 0.747 & 0.621 & 0.457 \\
\hline SB4VS2-03 & 38 & $\begin{array}{c}\text { Tk } 40 \text { Post Late Decant } \\
(5-6-08)\end{array}$ & $\mathrm{ccc}$ & targeted & 0.681 & -0.1386 & -0.1088 & -0.1841 & -0.3322 & 0.727 & 0.778 & 0.655 & 0.465 \\
\hline SB4VS2-04 & 32 & $\begin{array}{c}\text { Tk } 40 \text { Post Late Decant w } \\
\text { ARP (5-6-08) }\end{array}$ & $\mathrm{ccc}$ & targeted & 0.720 & -0.1921 & -0.1637 & -0.2565 & -0.3419 & 0.642 & 0.686 & 0.554 & 0.455 \\
\hline SB4VS2-05 & 35 & $\begin{array}{l}\text { Tk } 40 \text { Post Late Decant w } \\
\text { ARP (5-6-08) }\end{array}$ & $\mathrm{ccc}$ & targeted & 0.700 & -0.1845 & -0.1534 & -0.2249 & -0.3431 & 0.654 & 0.702 & 0.596 & 0.454 \\
\hline SB4VS2-06 & 38 & $\begin{array}{l}\text { Tk } 40 \text { Post Late Decant w } \\
\text { ARP }(5-6-08)\end{array}$ & $\mathrm{ccc}$ & targeted & 0.680 & -0.1559 & -0.1278 & -0.1813 & -0.3380 & 0.698 & 0.745 & 0.659 & 0.459 \\
\hline SB4VS2-07 & 32 & $\begin{array}{l}\text { Tk } 40 \text { Post Late Decant } w \\
\text { ARP }+2 \% \mathrm{Na} 2 \mathrm{O}(5-6-08) \\
\end{array}$ & $\mathrm{ccc}$ & targeted & 0.715 & -0.1775 & -0.1409 & -0.2017 & -0.3333 & 0.665 & 0.723 & 0.628 & 0.464 \\
\hline SB4VS2-08 & 35 & $\begin{array}{l}\text { Tk } 40 \text { Post Late Decant } w \\
\text { ARP }+2 \% \text { Na2O (5-6-08) }\end{array}$ & $\mathrm{ccc}$ & targeted & 0.695 & -0.1777 & -0.1442 & -0.1796 & -0.3373 & 0.664 & 0.717 & 0.661 & 0.460 \\
\hline SB4VS2-09 & 38 & $\begin{array}{l}\text { Tk } 40 \text { Post Late Decant } w \\
\text { ARP }+2 \% \text { Na2O (5-6-08) }\end{array}$ & $\mathrm{ccc}$ & targeted & 0.674 & -0.1535 & -0.1321 & -0.1593 & -0.3330 & 0.702 & 0.738 & 0.693 & 0.465 \\
\hline SB4VS2-01 & 32 & $\begin{array}{c}\text { Tk } 40 \text { Post Late Decant } \\
(5-6-08)\end{array}$ & quenched & targeted & 0.720 & -0.1748 & -0.1330 & -0.2524 & -0.3406 & 0.669 & 0.736 & 0.559 & 0.456 \\
\hline SB4VS2-02 & 35 & $\begin{array}{c}\text { Tk } 40 \text { Post Late Decant } \\
(5-6-08)\end{array}$ & quenched & targeted & 0.701 & -0.1640 & -0.1199 & -0.2209 & -0.3382 & 0.685 & 0.759 & 0.601 & 0.459 \\
\hline SB4VS2-03 & 38 & $\begin{array}{l}\text { Tk } 40 \text { Post Late Decant } \\
(5-6-08)\end{array}$ & quenched & targeted & 0.681 & -0.1239 & -0.0889 & -0.1636 & -0.3217 & 0.752 & 0.815 & 0.686 & 0.477 \\
\hline SB4VS2-04 & 32 & $\begin{array}{l}\text { Tk } 40 \text { Post Late Decant w } \\
\text { ARP (5-6-08) }\end{array}$ & quenched & targeted & 0.720 & -0.1963 & -0.1447 & -0.2260 & -0.3416 & 0.636 & 0.717 & 0.594 & 0.455 \\
\hline SB4VS2-05 & 35 & $\begin{array}{c}\text { Tk } 40 \text { Post Late Decant w } \\
\text { ARP (5-6-08) } \\
\end{array}$ & quenched & targeted & 0.700 & -0.1898 & -0.1471 & -0.2179 & -0.3573 & 0.646 & 0.713 & 0.606 & 0.439 \\
\hline SB4VS2-06 & 38 & $\begin{array}{c}\text { Tk } 40 \text { Post Late Decant w } \\
\text { ARP (5-6-08) } \\
\end{array}$ & quenched & targeted & 0.680 & -0.1373 & -0.1127 & -0.1728 & -0.3255 & 0.729 & 0.771 & 0.672 & 0.473 \\
\hline SB4VS2-07 & 32 & $\begin{array}{l}\text { Tk } 40 \text { Post Late Decant } w \\
\text { ARP }+2 \% \text { Na2O (5-6-08) }\end{array}$ & quenched & targeted & 0.715 & -0.1616 & -0.1265 & -0.1998 & -0.3214 & 0.689 & 0.747 & 0.631 & 0.477 \\
\hline SB4VS2-08 & 35 & $\begin{array}{l}\text { Tk } 40 \text { Post Late Decant } w \\
\text { ARP }+2 \% \text { Na2O (5-6-08) }\end{array}$ & quenched & targeted & 0.695 & -0.1650 & -0.1332 & -0.1780 & -0.3380 & 0.684 & 0.736 & 0.664 & 0.459 \\
\hline SB4VS2-09 & 38 & $\begin{array}{l}\text { Tk } 40 \text { Post Late Decant } w \\
\text { ARP }+2 \% \text { Na2O (5-6-08) }\end{array}$ & quenched & targeted & 0.674 & -0.1394 & -0.1195 & -0.1439 & -0.3270 & 0.725 & 0.759 & 0.718 & 0.471 \\
\hline SB4VS2-01 & 32 & $\begin{array}{c}\text { Tk } 40 \text { Post Late Decant } \\
(5-6-08)\end{array}$ & $\mathrm{ccc}$ & measured & 0.720 & -0.1790 & -0.1406 & -0.2446 & -0.3437 & 0.662 & 0.723 & 0.569 & 0.453 \\
\hline
\end{tabular}


Table 6 cont. Normalized PCTs by Glass ID/Compositional View for SB4/Second Decant Glasses with Frit 510

\begin{tabular}{|c|c|c|c|c|c|c|c|c|c|c|c|c|c|}
\hline Glass & WL \% & Sludge Case & $\begin{array}{c}\text { Heat } \\
\text { Treatment }\end{array}$ & $\begin{array}{l}\text { Compositional } \\
\text { View }\end{array}$ & $\begin{array}{l}\text { Nepheline } \\
\text { Assessment }\end{array}$ & $\begin{array}{c}\log \\
N L[B \\
(g / L)]\end{array}$ & $\begin{array}{c}\log \\
\mathrm{NL}[\mathrm{Li} \\
(\mathrm{g} / \mathrm{L})]\end{array}$ & $\begin{array}{c}\log \\
\mathrm{NL}[\mathrm{Na} \\
(\mathrm{g} / \mathrm{L})]\end{array}$ & $\begin{array}{c}\log \mathrm{NL} \\
{[\mathrm{Si}(\mathrm{g} / \mathrm{L})]}\end{array}$ & $\begin{array}{l}\mathrm{NL}[\mathrm{B} \\
(\mathrm{g} / \mathrm{L})]\end{array}$ & $\begin{array}{l}\mathrm{NL}[\mathrm{Li} \\
(\mathrm{g} / \mathrm{L})]\end{array}$ & $\begin{array}{c}\mathrm{NL}[\mathrm{Na} \\
(\mathrm{g} / \mathrm{L})]\end{array}$ & $\begin{array}{l}\mathrm{NL}[\mathrm{Si} \\
(\mathrm{g} / \mathrm{L})]\end{array}$ \\
\hline SB4VS2-02 & 35 & $\begin{array}{c}\text { Tk } 40 \text { Post Late Decant } \\
(5-6-08)\end{array}$ & $\mathrm{ccc}$ & measured & 0.697 & -0.1531 & -0.1240 & -0.2213 & -0.3493 & 0.703 & 0.752 & 0.601 & 0.447 \\
\hline SB4VS2-03 & 38 & $\begin{array}{c}\text { Tk } 40 \text { Post Late Decant } \\
(5-6-08)\end{array}$ & $\mathrm{ccc}$ & measured & 0.680 & -0.1304 & -0.0966 & -0.1838 & -0.3269 & 0.741 & 0.801 & 0.655 & 0.471 \\
\hline SB4VS2-04 & 32 & $\begin{array}{c}\text { Tk } 40 \text { Post Late Decant w } \\
\text { ARP (5-6-08) }\end{array}$ & $\mathrm{ccc}$ & measured & 0.717 & -0.1796 & -0.1525 & -0.2588 & -0.3377 & 0.661 & 0.704 & 0.551 & 0.460 \\
\hline SB4VS2-05 & 35 & $\begin{array}{l}\text { Tk } 40 \text { Post Late Decant w } \\
\text { ARP (5-6-08) }\end{array}$ & $\mathrm{ccc}$ & measured & 0.699 & -0.1809 & -0.1484 & -0.2282 & -0.3436 & 0.659 & 0.711 & 0.591 & 0.453 \\
\hline SB4VS2-06 & 38 & $\begin{array}{c}\text { Tk } 40 \text { Post Late Decant w } \\
\text { ARP (5-6-08) }\end{array}$ & $\mathrm{ccc}$ & measured & 0.680 & -0.1448 & -0.1098 & -0.1819 & -0.3399 & 0.717 & 0.777 & 0.658 & 0.457 \\
\hline SB4VS2-07 & 32 & $\begin{array}{l}\text { Tk } 40 \text { Post Late Decant } w \\
\text { ARP }+2 \% \mathrm{Na} 2 \mathrm{O}(5-6-08) \\
\end{array}$ & $\mathrm{ccc}$ & measured & 0.713 & -0.1676 & -0.1275 & -0.1994 & -0.3294 & 0.680 & 0.746 & 0.632 & 0.468 \\
\hline SB4VS2-08 & 35 & $\begin{array}{l}\text { Tk } 40 \text { Post Late Decant } w \\
\text { ARP }+2 \% \text { Na2O (5-6-08) }\end{array}$ & $\mathrm{ccc}$ & measured & 0.693 & -0.1635 & -0.1291 & -0.1827 & -0.3370 & 0.686 & 0.743 & 0.657 & 0.460 \\
\hline SB4VS2-09 & 38 & $\begin{array}{l}\text { Tk } 40 \text { Post Late Decant } w \\
\text { ARP }+2 \% \text { Na2O (5-6-08) }\end{array}$ & $\mathrm{ccc}$ & measured & 0.672 & -0.1462 & -0.1283 & -0.1658 & -0.3396 & 0.714 & 0.744 & 0.683 & 0.458 \\
\hline SB4VS2-01 & 32 & $\begin{array}{c}\text { Tk } 40 \text { Post Late Decant } \\
(5-6-08)\end{array}$ & quenched & measured & 0.720 & -0.1679 & -0.1248 & -0.2515 & -0.3390 & 0.679 & 0.750 & 0.560 & 0.458 \\
\hline SB4VS2-02 & 35 & $\begin{array}{l}\text { Tk } 40 \text { Post Late Decant } \\
(5-6-08)\end{array}$ & quenched & measured & 0.697 & -0.1594 & -0.1174 & -0.2350 & -0.3477 & 0.693 & 0.763 & 0.582 & 0.449 \\
\hline SB4VS2-03 & 38 & $\begin{array}{c}\text { Tk } 40 \text { Post Late Decant } \\
(5-6-08)\end{array}$ & quenched & measured & 0.680 & -0.1157 & -0.0767 & -0.1633 & -0.3165 & 0.766 & 0.838 & 0.687 & 0.483 \\
\hline SB4VS2-04 & 32 & $\begin{array}{c}\text { Tk } 40 \text { Post Late Decant w } \\
\text { ARP (5-6-08) } \\
\end{array}$ & quenched & measured & 0.717 & -0.1838 & -0.1335 & -0.2283 & -0.3375 & 0.655 & 0.735 & 0.591 & 0.460 \\
\hline SB4VS2-05 & 35 & $\begin{array}{l}\text { Tk } 40 \text { Post Late Decant w } \\
\text { ARP (5-6-08) }\end{array}$ & quenched & measured & 0.699 & -0.1861 & -0.1420 & -0.2212 & -0.3578 & 0.651 & 0.721 & 0.601 & 0.439 \\
\hline SB4VS2-06 & 38 & $\begin{array}{c}\text { Tk } 40 \text { Post Late Decant w } \\
\text { ARP (5-6-08) }\end{array}$ & quenched & measured & 0.680 & -0.1262 & -0.0946 & -0.1734 & -0.3275 & 0.748 & 0.804 & 0.671 & 0.470 \\
\hline SB4VS2-07 & 32 & $\begin{array}{l}\text { Tk } 40 \text { Post Late Decant } w \\
\text { ARP }+2 \% \text { Na2O (5-6-08) }\end{array}$ & quenched & measured & 0.713 & -0.1517 & -0.1131 & -0.1975 & -0.3174 & 0.705 & 0.771 & 0.635 & 0.482 \\
\hline SB4VS2-08 & 35 & $\begin{array}{l}\text { Tk } 40 \text { Post Late Decant } w \\
\text { ARP }+2 \% \text { Na2O (5-6-08) }\end{array}$ & quenched & measured & 0.693 & -0.1508 & -0.1180 & -0.1812 & -0.3378 & 0.707 & 0.762 & 0.659 & 0.459 \\
\hline SB4VS2-09 & 38 & $\begin{array}{l}\text { Tk } 40 \text { Post Late Decant w } \\
\text { ARP }+2 \% \text { Na2O (5-6-08) }\end{array}$ & quenched & measured & 0.672 & -0.1322 & -0.1157 & -0.1504 & -0.3336 & 0.738 & 0.766 & 0.707 & 0.464 \\
\hline SB4VS2-01 & 32 & $\begin{array}{c}\text { Tk } 40 \text { Post Late Decant } \\
(5-6-08) \\
\end{array}$ & $\mathrm{ccc}$ & measured bc & 0.722 & -0.1894 & -0.1546 & -0.2358 & -0.3482 & 0.647 & 0.700 & 0.581 & 0.449 \\
\hline SB4VS2-02 & 35 & $\begin{array}{c}\text { Tk } 40 \text { Post Late Decant } \\
(5-6-08)\end{array}$ & $\mathrm{ccc}$ & measured bc & 0.702 & -0.1534 & -0.1339 & -0.2157 & -0.3584 & 0.702 & 0.735 & 0.609 & 0.438 \\
\hline
\end{tabular}


Table 6 cont. Normalized PCTs by Glass ID/Compositional View for SB4/Second Decant Glasses with Frit 510

\begin{tabular}{|c|c|c|c|c|c|c|c|c|c|c|c|c|c|}
\hline Glass & WL $\%$ & Sludge Case & $\begin{array}{c}\text { Heat } \\
\text { Treatment }\end{array}$ & $\begin{array}{l}\text { Compositional } \\
\text { View }\end{array}$ & $\begin{array}{l}\text { Nepheline } \\
\text { Assessment }\end{array}$ & $\begin{array}{c}\log \\
\mathrm{NL}[\mathrm{B} \\
(\mathrm{g} / \mathrm{L})]\end{array}$ & $\begin{array}{c}\log \\
\mathrm{NL}[\mathrm{Li} \\
(\mathrm{g} / \mathrm{L})]\end{array}$ & $\begin{array}{c}\log \\
\mathrm{NL}[\mathrm{Na} \\
(\mathrm{g} / \mathrm{L})] \\
\end{array}$ & $\begin{array}{c}\log \mathrm{NL} \\
{[\mathrm{Si}(\mathrm{g} / \mathrm{L})]}\end{array}$ & $\begin{array}{l}\mathrm{NL}[\mathrm{B} \\
(\mathrm{g} / \mathrm{L})]\end{array}$ & $\begin{array}{l}\mathrm{NL}[\mathrm{Li} \\
(\mathrm{g} / \mathrm{L})]\end{array}$ & $\begin{array}{c}\mathrm{NL}[\mathrm{Na} \\
(\mathrm{g} / \mathrm{L})]\end{array}$ & $\begin{array}{l}\mathrm{NL}[\mathrm{Si} \\
(\mathrm{g} / \mathrm{L})]\end{array}$ \\
\hline SB4VS2-03 & 38 & $\begin{array}{c}\text { Tk } 40 \text { Post Late Decant } \\
(5-6-08)\end{array}$ & $\mathrm{ccc}$ & measured $b c$ & 0.681 & -0.1407 & -0.1107 & -0.1749 & -0.3314 & 0.723 & 0.775 & 0.668 & 0.466 \\
\hline SB4VS2-04 & 32 & $\begin{array}{c}\text { Tk } 40 \text { Post Late Decant w } \\
\text { ARP (5-6-08) } \\
\end{array}$ & $\mathrm{ccc}$ & measured bc & 0.719 & -0.1900 & -0.1666 & -0.2500 & -0.3422 & 0.646 & 0.681 & 0.562 & 0.455 \\
\hline SB4VS2-05 & 35 & $\begin{array}{l}\text { Tk } 40 \text { Post Late Decant w } \\
\text { ARP }(5-6-08)\end{array}$ & $\mathrm{ccc}$ & measured bc & 0.700 & -0.1912 & -0.1625 & -0.2193 & -0.3481 & 0.644 & 0.688 & 0.603 & 0.449 \\
\hline SB4VS2-06 & 38 & $\begin{array}{l}\text { Tk } 40 \text { Post Late Decant w } \\
\text { ARP (5-6-08) }\end{array}$ & $\mathrm{ccc}$ & measured bc & 0.682 & -0.1551 & -0.1238 & -0.1731 & -0.3444 & 0.700 & 0.752 & 0.671 & 0.452 \\
\hline SB4VS2-07 & 32 & $\begin{array}{l}\text { Tk } 40 \text { Post Late Decant } w \\
\text { ARP }+2 \% \text { Na2O (5-6-08) }\end{array}$ & $\mathrm{ccc}$ & measured bc & 0.715 & -0.1779 & -0.1415 & -0.1905 & -0.3339 & 0.664 & 0.722 & 0.645 & 0.464 \\
\hline SB4VS2-08 & 35 & $\begin{array}{l}\text { Tk } 40 \text { Post Late Decant } w \\
\text { ARP }+2 \% \text { Na2O (5-6-08) }\end{array}$ & $\mathrm{ccc}$ & measured bc & 0.695 & -0.1739 & -0.1431 & -0.1739 & -0.3415 & 0.670 & 0.719 & 0.670 & 0.456 \\
\hline SB4VS2-09 & 38 & $\begin{array}{l}\text { Tk } 40 \text { Post Late Decant w } \\
\text { ARP }+2 \% \text { Na2O (5-6-08) }\end{array}$ & $\mathrm{ccc}$ & measured bc & 0.677 & -0.1492 & -0.1392 & -0.1602 & -0.3487 & 0.709 & 0.726 & 0.691 & 0.448 \\
\hline SB4VS2-01 & 32 & $\begin{array}{c}\text { Tk } 40 \text { Post Late Decant } \\
(5-6-08) \\
\end{array}$ & quenched & measured bc & 0.722 & -0.1783 & -0.1389 & -0.2427 & -0.3435 & 0.663 & 0.726 & 0.572 & 0.453 \\
\hline SB4VS2-02 & 35 & $\begin{array}{c}\text { Tk } 40 \text { Post Late Decant } \\
(5-6-08)\end{array}$ & quenched & measured bc & 0.702 & -0.1598 & -0.1273 & -0.2294 & -0.3569 & 0.692 & 0.746 & 0.590 & 0.440 \\
\hline SB4VS2-03 & 38 & $\begin{array}{c}\text { Tk } 40 \text { Post Late Decant } \\
\text { (5-6-08) } \\
\end{array}$ & quenched & measured bc & 0.681 & -0.1261 & -0.0908 & -0.1545 & -0.3210 & 0.748 & 0.811 & 0.701 & 0.478 \\
\hline SB4VS2-04 & 32 & $\begin{array}{c}\text { Tk } 40 \text { Post Late Decant w } \\
\text { ARP (5-6-08) } \\
\end{array}$ & quenched & measured bc & 0.719 & -0.1942 & -0.1476 & -0.2194 & -0.3420 & 0.639 & 0.712 & 0.603 & 0.455 \\
\hline SB4VS2-05 & 35 & $\begin{array}{c}\text { Tk } 40 \text { Post Late Decant w } \\
\text { ARP (5-6-08) }\end{array}$ & quenched & measured bc & 0.700 & -0.1965 & -0.1561 & -0.2123 & -0.3623 & 0.636 & 0.698 & 0.613 & 0.434 \\
\hline SB4VS2-06 & 38 & $\begin{array}{l}\text { Tk } 40 \text { Post Late Decant w } \\
\text { ARP (5-6-08) }\end{array}$ & quenched & measured bc & 0.682 & -0.1366 & -0.1087 & -0.1645 & -0.3320 & 0.730 & 0.779 & 0.685 & 0.466 \\
\hline SB4VS2-07 & 32 & $\begin{array}{l}\text { Tk } 40 \text { Post Late Decant w } \\
\text { ARP }+2 \% \text { Na2O (5-6-08) }\end{array}$ & quenched & measured bc & 0.715 & -0.1620 & -0.1272 & -0.1886 & -0.3219 & 0.689 & 0.746 & 0.648 & 0.477 \\
\hline SB4VS2-08 & 35 & $\begin{array}{l}\text { Tk } 40 \text { Post Late Decant } \mathrm{w} \\
\mathrm{ARP}+2 \% \mathrm{Na} 2 \mathrm{O}(5-6-08) \\
\end{array}$ & quenched & measured bc & 0.695 & -0.1611 & -0.1321 & -0.1723 & -0.3423 & 0.690 & 0.738 & 0.673 & 0.455 \\
\hline SB4VS2-09 & 38 & $\begin{array}{l}\text { Tk } 40 \text { Post Late Decant } w \\
\text { ARP }+2 \% \text { Na2O }(5-6-08)\end{array}$ & quenched & measured bc & 0.677 & -0.1351 & -0.1267 & -0.1448 & -0.3427 & 0.733 & 0.747 & 0.716 & 0.454 \\
\hline
\end{tabular}




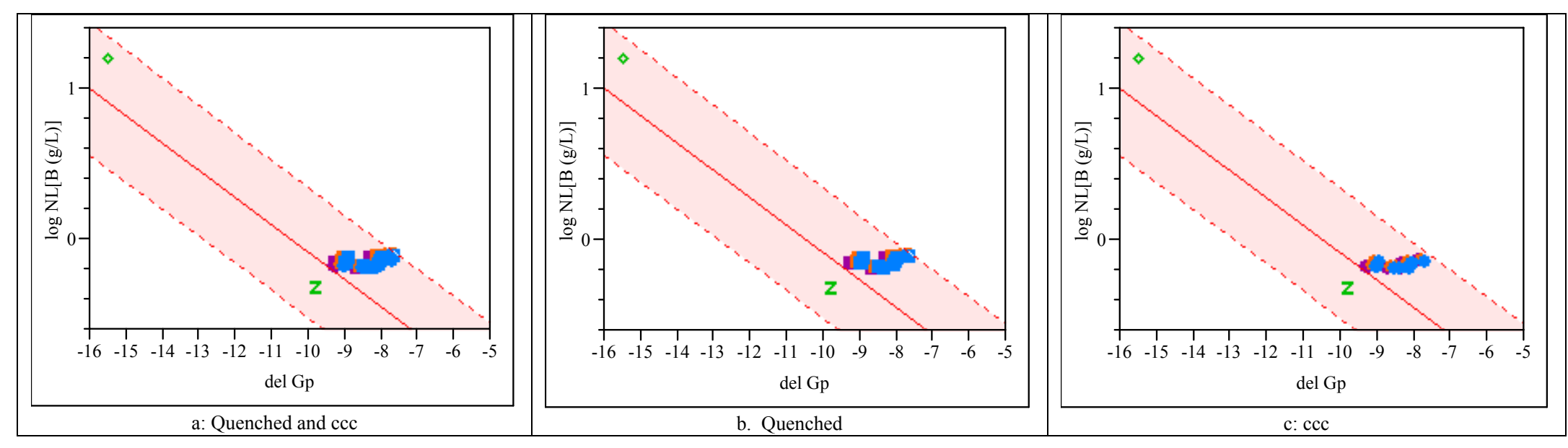

Figure 3. $\log \mathrm{NL}[\mathrm{B}]$ versus B del Gp model with a $95 \%$ confidence interval for individual PCTs. ${ }^{\mathrm{g}}$

${ }^{g}$ In plots "a" and "b" it appears that some glasses may be outside the $95 \%$ confidence band. A computational algorithm was developed to electronically compare the model predictions with the upper confidence bound. All of the data fall within the $95 \%$ confidence bands, thus eliminating the uncertainty. The size of the markers causes the edges to overlap the upper confidence bound. 
WSRC-STI-2008-00315

Revision 0

\subsection{Conclusions}

Frit 510 is a viable option for the processing of SB4 after a second Tank 40 decant with or without the addition of products from the Actinide Removal Process (ARP) as well as $2 \mathrm{wt} \% \mathrm{Na}_{2} \mathrm{O}$. The addition of ARP did not have any negative impacts on the acceptability and predictability of the variability study glasses.

All of the glasses that were selected for this study satisfy the PCCS criteria and are deemed processable and acceptable for the DWPF, except for the SB4VS2-03 (sludge-only at 38\% WL) target composition. This glass fails the $\mathrm{T}_{\mathrm{L}}$ criterion and would not be considered processable based on SME acceptability decisions.

The durabilities of all of the study glasses (both quenched and ccc) are well below that of the NL [B] of the reference EA glass $(16.695 \mathrm{~g} / \mathrm{L})$ and are predictable using the current PCCS models. Very little variation existed between the NL [B] of the quenched and ccc versions of the glasses. There is some evidence of a trend toward a less durable glass as WL increases for some of the sludge projections.

\subsection{References}

1. F.C. Raszewski, T.B. Edwards, D.K. Peeler, D.R. Best, I.A. Reamer, and R.J. Workman, "Initial SB4 Tank 40H Decant Variability Study with Frit 510," Savannah River National Laboratory, Aiken, SC, Report No. WSRC-STI-2008-00149, 2008.

2. $\quad$ F.C. Raszewski, M.E. Stone, T.B. Edwards, and D.K. Peeler, "The Impact of a Second Tank 40H Decant on the Frit 510 - SB4 System: Projected Operating Windows and CPC Processing," Savannah River National Laboratory, Aiken, SC, Report No. WSRC-TR-200800141, 2008.

3. J. Bricker, "SB4 Variability Studies for a Second Tank 40H Decant of up to 100,000 Gallons (TTR)," Savannah River National Laboratory, Aiken, SC, Report No. HLW-DWPF-TTR2008-0025, Rev. 0, 2008.

4. F.C. Raszewski and M.E. Stone, "Experimental Testing to Support Additional SB4 Tank 40 Decants (TT\&QAP)," Savannah River National Laboratory, Aiken, SC, Report No. WSRCSTI-2008-00254, Rev. 0, 2008.

5. S.L. Marra and C.M. Jantzen, "Characterization of Projected DWPF Glass Heat Treated to Simulate Canister Centerline Cooling," Westinghouse Savannah River Company, Aiken, SC, Report No. WSRC-TR-92-142, Rev. 1, 1993.

6. T.B. Edwards, "An Analytical Plan for Measuring the Chemical Compositions of Variability Study Glasses for SB4 after a Second Decant," Savannah River National Laboratory, Aiken, SC, Report No. SRNL-SCS-2008-00061 2008.

7. "Standard Test Methods for Determining Chemical Durability of Nuclear, Hazardous, and Mixed Waste Glasses and Multiphase Glass Ceramics: The Product Consistency Test (PCT)," ASTM International, West Conshohocken, PA, Report No. ASTM C 1285-02, 2002. 
8. T.B. Edwards, "An Analytical Plan for Measuring the PCT Solutions of Variability Study Glasses for SB4 after a Second Decant," Savannah River National Laboratory, Aiken, SC, Report No. SRNL-SCS-2008-00063, 2008.

9. C.M. Jantzen, J.B. Picket, K.G. Brown, T.B. Edwards, and D.C. Beam, "Process/Product Models for the Defense Waste Processing Facility (DWPF): Part I. Predicting Glass Durability from Composition Using a Thermodynamic Hydration Energy Reaction Model (THERMO)," Westinghouse Savannah River Company, Aiken, SC, Report No. WSRC-TR93-672, Rev. 1, 1995. 


\section{Appendix A:}

\section{Tables and Figures Supporting the Analysis of the Chemical Composition Measurements of the SB4 Variability Study Glasses in Support of a Second Decant}


WSRC-STI-2008-00315

Revision 0

This page intentionally left blank. 
Table A1. Measured Elemental Concentrations (wt\%) for Samples Prepared Using Lithium Metaborate (part 1)

\begin{tabular}{|c|c|c|c|c|c|c|c|c|c|c|c|c|}
\hline Glass ID & Blk & Seq. & Lab ID & $\mathrm{Al}(\mathrm{wt} \%)$ & $\mathrm{Ba}(\mathrm{wt} \%)$ & $\mathrm{Ca}(\mathrm{wt} \%)$ & Ce (wt\%) & $\mathrm{Cr}(\mathrm{wt} \%)$ & $\mathrm{Cu}(\mathrm{wt} \%)$ & $\mathrm{La}(\mathrm{wt} \%)$ & $\mathrm{Mg}$ (wt\%) & Mn (wt\%) \\
\hline Batch 1 & 1 & 1 & BCHLM11 & 2.52 & 0.124 & 0.847 & $<0.010$ & 0.073 & 0.304 & $<0.010$ & 0.8 & 1.29 \\
\hline Ustd & 1 & 2 & UstdLM11 & 2.14 & $<0.010$ & 0.913 & $<0.010$ & 0.161 & 0.005 & $<0.010$ & 0.674 & 2.14 \\
\hline SB4VS2-04 & 1 & 3 & A02LM11 & 4.45 & 0.024 & 0.639 & 0.012 & 0.041 & 0.019 & 0.013 & 0.527 & 1.57 \\
\hline SB4VS2-01 & 1 & 4 & A07LM21 & 4.58 & 0.015 & 0.663 & 0.014 & 0.042 & 0.021 & 0.012 & 0.55 & 1.57 \\
\hline SB4VS2-09 & 1 & 5 & A08LM21 & 5.29 & 0.024 & 0.735 & 0.025 & 0.039 & 0.02 & 0.015 & 0.61 & 1.78 \\
\hline SB4VS2-01 & 1 & 6 & A07LM11 & 4.54 & 0.016 & 0.655 & 0.014 & 0.043 & 0.021 & 0.013 & 0.562 & 1.52 \\
\hline SB4VS2-04 & 1 & 7 & A02LM21 & 4.34 & 0.024 & 0.628 & 0.012 & 0.041 & 0.019 & 0.014 & 0.541 & 1.47 \\
\hline SB4VS2-07 & 1 & 8 & A04LM11 & 4.32 & 0.013 & 0.639 & 0.015 & 0.038 & 0.019 & 0.014 & 0.499 & 1.46 \\
\hline SB4VS2-03 & 1 & 9 & A05LM21 & 5.33 & 0.023 & 0.784 & 0.024 & 0.042 & 0.021 & 0.014 & 0.622 & 1.8 \\
\hline SB4VS2-06 & 1 & 10 & A03LM11 & 5.24 & 0.022 & 0.749 & 0.019 & 0.041 & 0.021 & 0.013 & 0.616 & 1.81 \\
\hline SB4VS2-08 & 1 & 11 & A01LM11 & 4.71 & 0.025 & 0.675 & 0.021 & 0.038 & 0.02 & 0.013 & 0.569 & 1.66 \\
\hline Batch 1 & 1 & 12 & BCHLM12 & 2.52 & 0.129 & 0.83 & $<0.010$ & 0.077 & 0.303 & $<0.010$ & 0.838 & 1.29 \\
\hline Ustd & 1 & 13 & UstdLM12 & 2.07 & $<0.010$ & 0.902 & $<0.010$ & 0.171 & 0.005 & $<0.010$ & 0.718 & 2.06 \\
\hline SB4VS2-05 & 1 & 14 & A06LM21 & 4.8 & 0.024 & 0.697 & 0.021 & 0.042 & 0.02 & 0.014 & 0.598 & 1.66 \\
\hline SB4VS2-03 & 1 & 15 & A05LM11 & 5.38 & 0.027 & 0.77 & 0.022 & 0.047 & 0.022 & 0.015 & 0.695 & 1.76 \\
\hline SB4VS2-07 & 1 & 16 & A04LM21 & 4.29 & 0.015 & 0.616 & 0.017 & 0.043 & 0.022 & 0.015 & 0.566 & 1.48 \\
\hline SB4VS2-05 & 1 & 17 & A06LM11 & 4.88 & 0.024 & 0.703 & 0.021 & 0.041 & 0.021 & 0.013 & 0.591 & 1.65 \\
\hline $\begin{array}{l}\text { SB4VS2-02 } \\
\end{array}$ & 1 & 18 & A09LM21 & 5.1 & 0.027 & 0.715 & 0.013 & 0.04 & 0.022 & 0.015 & 0.637 & 1.78 \\
\hline SB4VS2-09 & 1 & 19 & A08LM11 & 5.16 & 0.024 & 0.745 & 0.02 & 0.041 & 0.019 & 0.015 & 0.641 & 1.84 \\
\hline SB4VS2-06 & 1 & 20 & A03LM21 & 5.24 & 0.024 & 0.747 & 0.019 & 0.043 & 0.022 & 0.013 & 0.642 & 1.82 \\
\hline SB4VS2-08 & 1 & 21 & A01LM21 & 4.75 & 0.026 & 0.674 & 0.022 & 0.041 & 0.021 & 0.014 & 0.601 & 1.61 \\
\hline SB4VS2-02 & 1 & 22 & A09LM11 & 5.32 & 0.029 & 1.11 & 0.018 & 0.039 & 0.022 & 0.016 & 0.683 & 1.71 \\
\hline Batch 1 & 1 & 23 & BCHLM13 & 2.51 & 0.135 & 0.839 & $<0.010$ & 0.08 & 0.311 & $<0.010$ & 0.874 & 1.33 \\
\hline Ustd & 1 & 24 & UstdLM13 & 2.09 & $<0.010$ & 0.925 & $<0.010$ & 0.174 & 0.006 & $<0.010$ & 0.73 & 2.21 \\
\hline Batch 1 & 2 & 1 & BCHLM21 & 2.5 & 0.124 & 0.815 & $<0.010$ & 0.073 & 0.296 & $<0.010$ & 0.817 & 1.25 \\
\hline Ustd & 2 & 2 & UstdLM21 & 2.13 & $<0.010$ & 0.891 & $<0.010$ & 0.162 & 0.007 & $<0.010$ & 0.685 & 2.1 \\
\hline SB4VS2-03 & 2 & 3 & A05LM22 & 5.35 & 0.023 & 0.768 & 0.026 & 0.041 & 0.023 & 0.013 & 0.624 & 1.74 \\
\hline SB4VS2-07 & 2 & 4 & A04LM22 & 4.29 & 0.013 & 0.617 & 0.018 & 0.04 & 0.023 & 0.014 & 0.527 & 1.41 \\
\hline SB4VS2-05 & 2 & 5 & A06LM12 & 4.88 & 0.021 & 0.693 & 0.022 & 0.038 & 0.023 & 0.012 & 0.553 & 1.61 \\
\hline SB4VS2-06 & 2 & 6 & A03LM12 & 5.26 & 0.022 & 0.736 & 0.021 & 0.041 & 0.023 & 0.013 & 0.627 & 1.7 \\
\hline SB4VS2-01 & 2 & 7 & A07LM12 & 4.53 & 0.015 & 0.64 & 0.015 & 0.043 & 0.023 & 0.012 & 0.574 & 1.39 \\
\hline SB4VS2-04 & 2 & 8 & A02LM22 & 4.34 & 0.024 & 0.619 & 0.013 & 0.041 & 0.021 & 0.014 & 0.541 & 1.38 \\
\hline SB4VS2-02 & 2 & 9 & A09LM22 & 5.03 & 0.024 & 0.706 & 0.015 & 0.037 & 0.023 & 0.014 & 0.613 & 1.6 \\
\hline SB4VS2-03 & 2 & 10 & A05LM12 & 5.34 & 0.024 & 0.766 & 0.023 & 0.044 & 0.024 & 0.014 & 0.66 & 1.68 \\
\hline SB4VS2-09 & 2 & 11 & A08LM22 & 5.28 & 0.024 & 0.729 & 0.027 & 0.039 & 0.022 & 0.015 & 0.624 & 1.67 \\
\hline Batch 1 & 2 & 12 & BCHLM22 & 2.49 & 0.125 & 0.825 & $<0.010$ & 0.074 & 0.302 & $<0.010$ & 0.821 & 1.23 \\
\hline Ustd & 2 & 13 & UstdLM22 & 2.09 & $<0.010$ & 0.89 & $<0.010$ & 0.164 & 0.008 & $<0.010$ & 0.694 & 2.07 \\
\hline SB4VS2-01 & 2 & 14 & A07LM22 & 4.56 & 0.015 & 0.65 & 0.015 & 0.042 & 0.023 & 0.012 & 0.563 & 1.52 \\
\hline SB4VS2-09 & 2 & 15 & A08LM12 & 5.17 & 0.022 & 0.744 & 0.022 & 0.038 & 0.021 & 0.014 & 0.609 & 1.7 \\
\hline SB4VS2-07 & 2 & 16 & A04LM12 & 4.29 & 0.012 & 0.634 & 0.017 & 0.038 & 0.022 & 0.014 & 0.511 & 1.41 \\
\hline SB4VS2-04 & 2 & 17 & A02LM12 & 4.43 & 0.023 & 0.629 & 0.013 & 0.041 & 0.021 & 0.013 & 0.536 & 1.45 \\
\hline
\end{tabular}


Table A1. Measured Elemental Concentrations (wt\%) for Samples Prepared Using Lithium Metaborate (part 1)

\begin{tabular}{|c|c|c|c|c|c|c|c|c|c|c|c|c|}
\hline Glass ID & Blk & Seq. & Lab ID & $\mathrm{Al}(\mathrm{wt} \%)$ & Ва (wt\%) & Ca (wt\%) & Ce (wt\%) & Cr (wt\%) & $\mathrm{Cu}(\mathrm{wt} \%)$ & $\mathrm{La}(\mathrm{wt} \%)$ & $\mathrm{Mg}(\mathrm{wt} \%)$ & Mn (wt\%) \\
\hline SB4VS2-02 & 2 & 18 & A09LM12 & 5.32 & 0.027 & 1.1 & 0.019 & 0.036 & 0.024 & 0.015 & 0.649 & 1.6 \\
\hline SB4VS2-06 & 2 & 19 & A03LM22 & 5.2 & 0.022 & 0.745 & 0.02 & 0.041 & 0.024 & 0.012 & 0.626 & 1.76 \\
\hline SB4VS2-08 & 2 & 20 & A01LM22 & 4.74 & 0.024 & 0.66 & 0.023 & 0.038 & 0.022 & 0.013 & 0.58 & 1.6 \\
\hline SB4VS2-08 & 2 & 21 & A01LM12 & 4.68 & 0.024 & 0.663 & 0.022 & 0.037 & 0.022 & 0.013 & 0.572 & 1.56 \\
\hline SB4VS2-05 & 2 & 22 & A06LM22 & 4.83 & 0.023 & 0.687 & 0.022 & 0.04 & 0.022 & 0.013 & 0.59 & 1.65 \\
\hline Batch 1 & 2 & 23 & BCHLM23 & 2.5 & 0.127 & 0.829 & $<0.010$ & 0.075 & 0.303 & $<0.010$ & 0.827 & 1.27 \\
\hline Ustd & 2 & 24 & UstdLM23 & 2.05 & $<0.010$ & 0.901 & $<0.010$ & 0.164 & 0.008 & $<0.010$ & 0.697 & 2.02 \\
\hline Batch 1 & 3 & . & Batch glass & 2.55 & 0.121 & 0.821 & $<0.010$ & 0.072 & 0.298 & $<0.010$ & 0.784 & 1.32 \\
\hline Ustd & 3 & . & U glass & 2.12 & $<0.010$ & 0.881 & $<0.010$ & 0.163 & $<0.010$ & $<0.010$ & 0.682 & 2.07 \\
\hline SB4VS2-09 & 3 & . & A08 11 & 5.33 & 0.023 & 0.729 & 0.02 & 0.037 & 0.021 & 0.014 & 0.587 & 1.8 \\
\hline SB4VS2-09 & 3 & . & A08 12 & 5.36 & 0.023 & 0.737 & 0.02 & 0.037 & 0.021 & 0.014 & 0.594 & 1.81 \\
\hline SB4VS2-09 & 3 & . & A08 21 & 5.28 & 0.024 & 0.715 & 0.025 & 0.039 & 0.021 & 0.015 & 0.615 & 1.83 \\
\hline SB4VS2-09 & 3 & . & A08 22 & 5.32 & 0.025 & 0.715 & 0.025 & 0.039 & 0.021 & 0.015 & 0.623 & 1.84 \\
\hline SB4VS2-02 & 3 & . & A09 11 & 5.37 & 0.027 & 1.07 & 0.017 & 0.035 & 0.023 & 0.015 & 0.625 & 1.74 \\
\hline SB4VS2-02 & 3 & . & A09 12 & 5.29 & 0.027 & 1.07 & 0.017 & 0.035 & 0.023 & 0.014 & 0.627 & 1.76 \\
\hline SB4VS2-02 & 3 & . & A09 21 & 5.2 & 0.025 & 0.705 & 0.013 & 0.037 & 0.023 & 0.014 & 0.604 & 1.83 \\
\hline SB4VS2-02 & 3 & . & A09 22 & 5.19 & 0.025 & 0.693 & 0.013 & 0.037 & 0.023 & 0.014 & 0.594 & 1.78 \\
\hline Batch 1 & 3 & . & Batch glass & 2.58 & 0.118 & 0.799 & $<0.010$ & 0.07 & 0.29 & $<0.010$ & 0.773 & 1.37 \\
\hline Ustd & 3 & . & U glass & 2.13 & $<0.010$ & 0.886 & $<0.010$ & 0.162 & $<0.010$ & $<0.010$ & 0.684 & 2.3 \\
\hline
\end{tabular}


Table A1. Measured Elemental Concentrations (wt\%) for Samples Prepared Using Lithium Metaborate (part 2)

\begin{tabular}{|c|c|c|c|c|c|c|c|c|c|c|c|}
\hline Glass ID & Blk & Seq. & Lab ID & $\mathrm{Na}(\mathrm{wt} \%)$ & $\mathrm{Ni}(\mathrm{wt} \%)$ & $\mathrm{Pb}(\mathrm{wt} \%)$ & S (wt\%) & Si (wt\%) & $\mathrm{Ti}(\mathrm{wt} \%)$ & $\mathrm{U}(\mathrm{wt} \%)$ & $\mathrm{Zr}(\mathrm{wt} \%)$ \\
\hline Batch 1 & 1 & 1 & BCHLM11 & 7.06 & 0.54 & $<0.010$ & 0.012 & 23.3 & 0.381 & $<0.100$ & 0.062 \\
\hline Ustd & 1 & 2 & UstdLM11 & 8.86 & 0.762 & $<0.010$ & 0.012 & 21.2 & 0.542 & 1.91 & $<0.010$ \\
\hline SB4VS2-04 & 1 & 3 & A02LM11 & 7.97 & 0.393 & 0.019 & 0.069 & 23 & 0.251 & 2.39 & 0.021 \\
\hline SB4VS2-01 & 1 & 4 & A07LM21 & 7.63 & 0.387 & 0.021 & 0.062 & 22.9 & 0.017 & 2.44 & 0.017 \\
\hline SB4VS2-09 & 1 & 5 & A08LM21 & 8.93 & 0.465 & 0.023 & 0.09 & 21.1 & 0.275 & 2.77 & 0.023 \\
\hline SB4VS2-01 & 1 & 6 & A07LM11 & 7.55 & 0.401 & 0.022 & 0.064 & 22.7 & 0.017 & 2.4 & 0.018 \\
\hline SB4VS2-04 & 1 & 7 & A02LM21 & 7.92 & 0.4 & 0.019 & 0.074 & 22.3 & 0.258 & 2.34 & 0.022 \\
\hline SB4VS2-07 & 1 & 8 & A04LM11 & 8.32 & 0.358 & 0.018 & 0.068 & 22.6 & 0.241 & 2.28 & 0.021 \\
\hline SB4VS2-03 & 1 & 9 & A05LM21 & 7.85 & 0.406 & 0.02 & 0.074 & 20.7 & 0.018 & 2.85 & 0.022 \\
\hline SB4VS2-06 & 1 & 10 & A03LM11 & 8.3 & 0.455 & 0.029 & 0.09 & 21 & 0.296 & 2.8 & 0.021 \\
\hline SB4VS2-08 & 1 & 11 & A01LM11 & 8.6 & 0.418 & 0.023 & 0.086 & 22 & 0.276 & 2.53 & 0.019 \\
\hline Batch 1 & 1 & 12 & BCHLM12 & 6.85 & 0.563 & $<0.010$ & 0.016 & 23.3 & 0.391 & $<0.100$ & 0.063 \\
\hline Ustd & 1 & 13 & UstdLM12 & 8.87 & 0.805 & $<0.010$ & 0.012 & 21 & 0.561 & 1.89 & $<0.010$ \\
\hline SB4VS2-05 & 1 & 14 & A06LM21 & 8.09 & 0.448 & 0.022 & 0.081 & 21.8 & 0.281 & 2.53 & 0.02 \\
\hline SB4VS2-03 & 1 & 15 & A05LM11 & 7.97 & 0.468 & 0.024 & 0.078 & 20.7 & 0.02 & 2.85 & 0.024 \\
\hline SB4VS2-07 & 1 & 16 & A04LM21 & 8.35 & 0.42 & 0.023 & 0.08 & 22.5 & 0.271 & 2.26 & 0.022 \\
\hline SB4VS2-05 & 1 & 17 & A06LM11 & 8.2 & 0.434 & 0.02 & 0.081 & 21.8 & 0.275 & 2.57 & 0.02 \\
\hline SB4VS2-02 & 1 & 18 & A09LM21 & 7.95 & 0.457 & 0.022 & 0.075 & 22.4 & 0.018 & 2.73 & 0.023 \\
\hline SB4VS2-09 & 1 & 19 & A08LM11 & 8.92 & 0.469 & 0.023 & 0.091 & 21.2 & 0.281 & 2.76 & 0.026 \\
\hline SB4VS2-06 & 1 & 20 & A03LM21 & 8.26 & 0.468 & 0.03 & 0.091 & 21 & 0.303 & 2.83 & 0.021 \\
\hline SB4VS2-08 & 1 & 21 & A01LM21 & 8.79 & 0.449 & 0.024 & 0.089 & 21.7 & 0.287 & 2.47 & 0.019 \\
\hline SB4VS2-02 & 1 & 22 & A09LM11 & 8.02 & 0.441 & 0.021 & 0.073 & 21.7 & 0.018 & 2.65 & 0.023 \\
\hline Batch 1 & 1 & 23 & BCHLM13 & 6.72 & 0.584 & $<0.010$ & 0.016 & 23.6 & 0.404 & $<0.100$ & 0.065 \\
\hline Ustd & 1 & 24 & UstdLM13 & 8.85 & 0.815 & $<0.010$ & 0.014 & 21.5 & 0.576 & 1.93 & $<0.010$ \\
\hline Batch 1 & 2 & 1 & BCHLM21 & 6.97 & 0.545 & $<0.010$ & 0.012 & 23.1 & 0.381 & $<0.100$ & 0.061 \\
\hline Ustd & 2 & 2 & UstdLM21 & 8.76 & 0.773 & $<0.010$ & 0.015 & 21.1 & 0.548 & 1.92 & $<0.010$ \\
\hline SB4VS2-03 & 2 & 3 & A05LM22 & 7.85 & 0.41 & 0.02 & 0.069 & 20.5 & 0.018 & 2.85 & 0.021 \\
\hline SB4VS2-07 & 2 & 4 & A04LM22 & 8.32 & 0.396 & 0.019 & 0.073 & 22.2 & 0.26 & 2.24 & 0.021 \\
\hline SB4VS2-05 & 2 & 5 & A06LM12 & 8.07 & 0.409 & 0.017 & 0.079 & 21.6 & 0.264 & 2.52 & 0.019 \\
\hline SB4VS2-06 & 2 & 6 & A03LM12 & 8.27 & 0.464 & 0.03 & 0.089 & 20.8 & 0.301 & 2.76 & 0.021 \\
\hline SB4VS2-01 & 2 & 7 & A07LM12 & 7.5 & 0.408 & 0.022 & 0.064 & 22.1 & 0.017 & 2.35 & 0.018 \\
\hline SB4VS2-04 & 2 & 8 & A02LM22 & 7.87 & 0.409 & 0.019 & 0.075 & 22 & 0.261 & 2.29 & 0.022 \\
\hline SB4VS2-02 & 2 & 9 & A09LM22 & 7.76 & 0.439 & 0.02 & 0.071 & 21.5 & 0.017 & 2.6 & 0.022 \\
\hline SB4VS2-03 & 2 & 10 & A05LM12 & 7.79 & 0.445 & 0.021 & 0.075 & 20.3 & 0.019 & 2.79 & 0.023 \\
\hline SB4VS2-09 & 2 & 11 & A08LM22 & 8.83 & 0.474 & 0.023 & 0.094 & 20.7 & 0.279 & 2.71 & 0.023 \\
\hline Batch 1 & 2 & 12 & BCHLM22 & 6.57 & 0.55 & $<0.010$ & 0.012 & 23 & 0.384 & $<0.100$ & 0.062 \\
\hline Ustd & 2 & 13 & UstdLM22 & 8.76 & 0.782 & $<0.010$ & 0.013 & 21 & 0.559 & 1.9 & $<0.010$ \\
\hline SB4VS2-01 & 2 & 14 & A07LM22 & 7.52 & 0.402 & 0.021 & 0.066 & 22.7 & 0.017 & 2.4 & 0.018 \\
\hline SB4VS2-09 & 2 & 15 & A08LM12 & 8.78 & 0.447 & 0.021 & 0.087 & 20.8 & 0.271 & 2.7 & 0.024 \\
\hline SB4VS2-07 & 2 & 16 & A04LM12 & 8.17 & 0.367 & 0.017 & 0.073 & 22.5 & 0.246 & 2.24 & 0.021 \\
\hline SB4VS2-04 & 2 & 17 & A02LM12 & 7.85 & 0.401 & 0.018 & 0.071 & 22.5 & 0.258 & 2.33 & 0.022 \\
\hline
\end{tabular}


Table A1. Measured Elemental Concentrations (wt\%) for Samples Prepared Using Lithium Metaborate (part 2)

\begin{tabular}{||c|c|c|c|c|c|c|c|c|c|c|c||}
\hline \hline Glass ID & Blk & Seq. & Lab ID & $\mathrm{Na}(\mathrm{wt} \%)$ & $\mathrm{Ni}(\mathrm{wt} \%)$ & $\mathrm{Pb}(\mathrm{wt} \%)$ & $\mathrm{S}(\mathrm{wt} \%)$ & $\mathrm{Si}(\mathrm{wt} \%)$ & $\mathrm{Ti}(\mathrm{wt} \%)$ & $\mathrm{U}(\mathrm{wt} \%)$ & $\mathrm{Zr}(\mathrm{wt} \%)$ \\
\hline SB4VS2-02 & 2 & 18 & A09LM12 & 7.96 & 0.419 & 0.019 & 0.067 & 21.4 & 0.018 & 2.6 & 0.022 \\
\hline SB4VS2-06 & 2 & 19 & A03LM22 & 8.1 & 0.454 & 0.028 & 0.088 & 20.7 & 0.295 & 2.75 & 0.02 \\
\hline SB4VS2-08 & 2 & 20 & A01LM22 & 8.64 & 0.427 & 0.022 & 0.086 & 21.6 & 0.274 & 2.47 & 0.018 \\
\hline SB4VS2-08 & 2 & 21 & A01LM12 & 8.46 & 0.421 & 0.021 & 0.08 & 21.5 & 0.273 & 2.47 & 0.018 \\
\hline SB4VS2-05 & 2 & 22 & A06LM22 & 8.05 & 0.442 & 0.02 & 0.081 & 21.8 & 0.275 & 2.55 & 0.02 \\
\hline Batch 1 & 2 & 23 & BCHLM23 & 6.73 & 0.556 & $<0.010$ & 0.013 & 23.1 & 0.389 & $<0.100$ & 0.063 \\
\hline Ustd & 2 & 24 & UstdLM23 & 8.66 & 0.785 & $<0.010$ & 0.012 & 20.6 & 0.558 & 1.85 & $<0.010$ \\
\hline Batch 1 & 3 &. & Batch glass & 6.64 & 0.525 & $<0.010$ & 0.014 & 22.4 & 0.37 & $<0.100$ & 0.059 \\
\hline Ustd & 3 &. & U glass & 8.81 & 0.782 & $<0.010$ & 0.015 & 20.9 & 0.549 & 1.91 & $<0.010$ \\
\hline SB4VS2-09 & 3 &. & A08 11 & 8.94 & 0.441 & 0.021 & 0.088 & 21.4 & 0.262 & 2.79 & 0.023 \\
\hline SB4VS2-09 & 3 &. & A08 12 & 8.98 & 0.445 & 0.021 & 0.085 & 21.2 & 0.265 & 2.81 & 0.023 \\
\hline SB4VS2-09 & 3 &. & A08 21 & 9.02 & 0.469 & 0.023 & 0.091 & 21.5 & 0.273 & 2.83 & 0.022 \\
\hline SB4VS2-09 & 3 &. & A08 22 & 8.95 & 0.472 & 0.024 & 0.094 & 20.8 & 0.274 & 2.81 & 0.022 \\
\hline SB4VS2-02 & 3 &. & A0911 & 8 & 0.412 & 0.018 & 0.069 & 22.7 & 0.017 & 2.71 & 0.021 \\
\hline SB4VS2-02 & 3 &. & A09 12 & 8.04 & 0.411 & 0.018 & 0.068 & 22.8 & 0.017 & 2.72 & 0.02 \\
\hline SB4VS2-02 & 3 &. & A09 21 & 7.97 & 0.436 & 0.02 & 0.074 & 22.5 & 0.017 & 2.8 & 0.02 \\
\hline SB4VS2-02 & 3 &. & A09 22 & 8.07 & 0.429 & 0.019 & 0.074 & 22.8 & 0.017 & 2.8 & 0.02 \\
\hline Batch 1 & 3 &. & Batch glass & 6.79 & 0.516 & $<0.010$ & 0.018 & 23.1 & 0.362 & $<0.100$ & 0.059 \\
\hline Ustd & 3 &. & U glass & 8.93 & 0.773 & $<0.010$ & 0.013 & 21.2 & 0.547 & 2 & $<0.010$ \\
\hline
\end{tabular}




\section{Table A2. Measured Elemental Concentrations (wt\%)} for Samples Prepared Using Peroxide Fusion

\begin{tabular}{|c|c|c|c|c|c|c|}
\hline Glass ID & Block & Sequence & Lab ID & B (wt\%) & Fe (wt\%) & Li (wt\%) \\
\hline Batch 1 & 1 & 1 & BCHPF11 & 2.44 & 9.16 & 2.00 \\
\hline Ustd & 1 & 2 & UstdPF11 & 2.83 & 7.32 & 2.40 \\
\hline SB4VS2-02 & 1 & 3 & A09PF11 & 2.80 & 8.79 & 1.43 \\
\hline SB4VS2-04 & 1 & 4 & A02PF21 & 2.88 & 6.47 & 2.48 \\
\hline SB4VS2-05 & 1 & 5 & A06PF21 & 2.81 & 7.21 & 2.39 \\
\hline SB4VS2-04 & 1 & 6 & A02PF11 & 2.91 & 6.68 & 2.46 \\
\hline SB4VS2-05 & 1 & 7 & A06PF11 & 2.79 & 7.63 & 2.38 \\
\hline SB4VS2-01 & 1 & 8 & A07PF11 & 2.93 & 7.40 & 2.49 \\
\hline SB4VS2-01 & 1 & 9 & A07PF21 & 2.90 & 6.74 & 2.50 \\
\hline SB4VS2-07 & 1 & 10 & A04PF11 & 2.86 & 6.56 & 2.43 \\
\hline SB4VS2-06 & 1 & 11 & A03PF11 & 2.61 & 7.65 & 2.20 \\
\hline Batch 1 & 1 & 12 & BCHPF12 & 2.32 & 8.92 & 2.02 \\
\hline Ustd & 1 & 13 & UstdPF12 & 2.69 & 7.07 & 2.38 \\
\hline SB4VS2-09 & 1 & 14 & A08PF11 & 2.57 & 7.03 & 2.23 \\
\hline SB4VS2-06 & 1 & 15 & A03PF21 & 2.63 & 7.65 & 2.26 \\
\hline SB4VS2-08 & 1 & 16 & A01PF21 & 2.72 & 6.67 & 2.36 \\
\hline SB4VS2-08 & 1 & 17 & A01PF11 & 2.72 & 6.80 & 2.33 \\
\hline SB4VS2-09 & 1 & 18 & A08PF21 & 2.61 & 7.14 & 2.29 \\
\hline SB4VS2-02 & 1 & 19 & A09PF21 & 2.68 & 6.90 & 2.33 \\
\hline SB4VS2-03 & 1 & 20 & A05PF11 & 2.67 & 8.04 & 2.26 \\
\hline SB4VS2-03 & 1 & 21 & A05PF21 & 2.63 & 8.02 & 2.24 \\
\hline SB4VS2-07 & 1 & 22 & A04PF21 & 2.91 & 6.33 & 2.51 \\
\hline Batch 1 & 1 & 23 & BCHPF13 & 2.33 & 8.97 & 2.00 \\
\hline Ustd & 1 & 24 & UstdPF13 & 2.68 & 6.98 & 2.37 \\
\hline Batch 1 & 2 & 1 & BCHPF21 & 2.42 & 8.90 & 1.97 \\
\hline Ustd & 2 & 2 & UstdPF21 & 2.80 & 7.18 & 2.39 \\
\hline SB4VS2-01 & 2 & 3 & A07PF22 & 2.91 & 6.55 & 2.46 \\
\hline SB4VS2-06 & 2 & 4 & A03PF22 & 2.64 & 7.53 & 2.22 \\
\hline SB4VS2-07 & 2 & 5 & A04PF22 & 2.93 & 6.40 & 2.48 \\
\hline SB4VS2-04 & 2 & 6 & A02PF22 & 2.88 & 6.35 & 2.45 \\
\hline SB4VS2-09 & 2 & 7 & A08PF12 & 2.62 & 7.19 & 2.24 \\
\hline SB4VS2-02 & 2 & 8 & A09PF12 & 2.72 & 8.30 & 1.40 \\
\hline SB4VS2-07 & 2 & 9 & A04PF12 & 2.86 & 6.48 & 2.38 \\
\hline SB4VS2-05 & 2 & 10 & A06PF12 & 2.84 & 7.81 & 2.41 \\
\hline SB4VS2-09 & 2 & 11 & A08PF22 & 2.64 & 7.24 & 2.28 \\
\hline Batch 1 & 2 & 12 & BCHPF22 & 2.32 & 8.78 & 1.98 \\
\hline Ustd & 2 & 13 & UstdPF22 & 2.72 & 7.06 & 2.37 \\
\hline SB4VS2-06 & 2 & 14 & A03PF12 & 2.63 & 7.63 & 2.16 \\
\hline SB4VS2-03 & 2 & 15 & A05PF22 & 2.67 & 8.04 & 2.22 \\
\hline SB4VS2-08 & 2 & 16 & A01PF22 & 2.74 & 6.69 & 2.33 \\
\hline SB4VS2-08 & 2 & 17 & A01PF12 & 2.76 & 6.86 & 2.31 \\
\hline SB4VS2-01 & 2 & 18 & A07PF12 & 2.90 & 7.22 & 2.47 \\
\hline SB4VS2-03 & 2 & 19 & A05PF12 & 2.61 & 7.73 & 2.24 \\
\hline SB4VS2-04 & 2 & 20 & A02PF12 & 2.82 & 6.43 & 2.46 \\
\hline SB4VS2-02 & 2 & 21 & A09PF22 & 2.65 & 6.82 & 2.31 \\
\hline SB4VS2-05 & 2 & 22 & A06PF22 & 2.77 & 6.98 & 2.37 \\
\hline Batch 1 & 2 & 23 & BCHPF23 & 2.32 & 8.98 & 1.98 \\
\hline Ustd & 2 & 24 & UstdPF23 & 2.70 & 7.03 & 2.37 \\
\hline Batch 1 & 3 & 1 & Batch 1 & 2.47 & 8.70 & 2.01 \\
\hline Ustd & 3 & 2 & Ustd & 2.83 & 8.65 & 1.38 \\
\hline SB4VS2-09 & 3 & 3 & A08 11 & 2.68 & 7.35 & 2.31 \\
\hline SB4VS2-09 & 3 & 4 & A08 12 & 2.72 & 7.41 & 2.33 \\
\hline SB4VS2-09 & 3 & 5 & A08 21 & 2.72 & 7.47 & 2.31 \\
\hline SB4VS2-09 & 3 & 6 & A08 22 & 2.65 & 7.55 & 2.28 \\
\hline SB4VS2-02 & 3 & 7 & A09 11 & 2.89 & 7.36 & 2.48 \\
\hline SB4VS2-02 & 3 & 8 & A09 12 & 2.84 & 7.40 & 2.45 \\
\hline SB4VS2-02 & 3 & 9 & A09 21 & 2.84 & 7.67 & 2.41 \\
\hline SB4VS2-02 & 3 & 10 & A09 22 & 2.88 & 7.45 & 2.43 \\
\hline Batch 1 & 3 & 11 & Batch 1 & 2.41 & 8.98 & 2.03 \\
\hline Ustd & 3 & 12 & Ustd & 2.85 & 9.30 & 1.39 \\
\hline
\end{tabular}


Table A3: LM and PF Reruns

\section{(Measurements are in wt\% elementals.)}

\begin{tabular}{|c|c|c|c|c|c|c|c|c|c|c|c|c|c|c|c|c|c|c|c|c|}
\hline Solution ID & Al & B & Ba & $\mathbf{C a}$ & Ce & $\mathrm{Cr}$ & $\mathbf{C u}$ & $\mathbf{F e}$ & La & $\mathbf{L i}$ & Mg & Mn & $\mathrm{Na}$ & $\mathbf{N i}$ & $\mathbf{P b}$ & $\mathrm{S}$ & $\mathrm{Si}$ & $\mathbf{T i}$ & $\mathbf{U}$ & $\mathbf{Z r}$ \\
\hline Batch glass & 2.55 & 2.47 & 0.121 & 0.821 & $<0.010$ & 0.072 & 0.298 & 8.70 & $<0.010$ & 2.01 & 0.784 & 1.32 & 6.64 & 0.525 & $<0.010$ & 0.014 & 22.4 & 0.370 & $<0.100$ & 0.059 \\
\hline U glass & 2.12 & 2.83 & $<0.010$ & 0.881 & $<0.010$ & 0.163 & $<0.010$ & 8.65 & $<0.010$ & 1.38 & 0.682 & 2.07 & 8.81 & 0.782 & $<0.010$ & 0.015 & 20.9 & 0.549 & 1.91 & $<0.010$ \\
\hline A08 11 & 5.33 & 2.68 & 0.023 & 0.729 & 0.020 & 0.037 & 0.021 & 7.35 & 0.014 & 2.31 & 0.587 & 1.80 & 8.94 & 0.441 & 0.021 & 0.088 & 21.4 & 0.262 & 2.79 & 0.023 \\
\hline A08 12 & 5.36 & 2.72 & 0.023 & 0.737 & 0.020 & 0.037 & 0.021 & 7.41 & 0.014 & 2.33 & 0.594 & 1.81 & 8.98 & 0.445 & 0.021 & 0.085 & 21.2 & 0.265 & 2.81 & 0.023 \\
\hline A08 21 & 5.28 & 2.72 & 0.024 & 0.715 & 0.025 & 0.039 & 0.021 & 7.47 & 0.015 & 2.31 & 0.615 & 1.83 & 9.02 & 0.469 & 0.023 & 0.091 & 21.5 & 0.273 & 2.83 & 0.022 \\
\hline A08 22 & 5.32 & 2.65 & 0.025 & 0.715 & 0.025 & 0.039 & 0.021 & 7.55 & 0.015 & 2.28 & 0.623 & 1.84 & 8.95 & 0.472 & 0.024 & 0.094 & 20.8 & 0.274 & 2.81 & 0.022 \\
\hline A09 11 & 5.37 & 2.89 & 0.027 & 1.07 & 0.017 & 0.035 & 0.023 & 7.36 & 0.015 & 2.48 & 0.625 & 1.74 & 8.00 & 0.412 & 0.018 & 0.069 & 22.7 & 0.017 & 2.71 & 0.021 \\
\hline A09 12 & 5.29 & 2.84 & 0.027 & 1.07 & 0.017 & 0.035 & 0.023 & 7.40 & 0.014 & 2.45 & 0.627 & 1.76 & 8.04 & 0.411 & 0.018 & 0.068 & 22.8 & 0.017 & 2.72 & 0.020 \\
\hline A09 21 & 5.20 & 2.84 & 0.025 & 0.705 & 0.013 & 0.037 & 0.023 & 7.67 & 0.014 & 2.41 & 0.604 & 1.83 & 7.97 & 0.436 & 0.020 & 0.074 & 22.5 & 0.017 & 2.80 & 0.020 \\
\hline A09 22 & 5.19 & 2.88 & 0.025 & 0.693 & 0.013 & 0.037 & 0.023 & 7.45 & 0.014 & 2.43 & 0.594 & 1.78 & 8.07 & 0.429 & 0.019 & 0.074 & 22.8 & 0.017 & 2.80 & 0.020 \\
\hline Batch glass & 2.58 & 2.41 & 0.118 & 0.799 & $<0.010$ & 0.070 & 0.290 & 8.98 & $<0.010$ & 2.03 & 0.773 & 1.37 & 6.79 & 0.516 & $<0.010$ & 0.018 & 23.1 & 0.362 & $<0.100$ & 0.059 \\
\hline U glass & 2.13 & 2.85 & $<0.010$ & 0.886 & $<0.010$ & 0.162 & $<0.010$ & 9.30 & $<0.010$ & 1.39 & 0.684 & 2.30 & 8.93 & 0.773 & $<0.010$ & 0.013 & 21.2 & 0.547 & 2.00 & $<0.010$ \\
\hline
\end{tabular}


WSRC-STI-2008-00315

Revision 0

Table A4. Average Measured and Bias-Corrected Chemical Compositions Versus Targeted Compositions by Oxide by SB4-Decant Variability Study Glass

\begin{tabular}{|c|c|c|c|c|c|c|c|c|}
\hline & & & Measured & & & & & \\
\hline Glass & & Measured & Bias-Corrected & Targeted & Diff of & Diff of & \% Diff of & $\%$ Diff of \\
\hline ID & Oxide & $(\mathrm{wt} \%)$ & $(\mathrm{wt} \%)$ & (wt\%) & Measured & Meas BC & Measured & Meas BC \\
\hline SB4VS2-01 & $\mathrm{Al}_{2} \mathrm{O}_{3}$ & 8.6019 & 8.8575 & 8.6332 & -0.0313 & 0.2243 & $-0.4 \%$ & $2.6 \%$ \\
\hline SB4VS2-01 & $\mathrm{B}_{2} \mathrm{O}_{3}$ & 9.3699 & 9.5962 & 9.5200 & -0.1501 & 0.0762 & $-1.6 \%$ & $0.8 \%$ \\
\hline SB4VS2-01 & $\mathrm{BaO}$ & 0.0170 & 0.0181 & 0.0249 & -0.0079 & -0.0068 & $-31.6 \%$ & $-27.4 \%$ \\
\hline SB4VS2-01 & $\mathrm{CaO}$ & 0.9123 & 0.9574 & 0.9527 & -0.0404 & 0.0047 & $-4.2 \%$ & $0.5 \%$ \\
\hline SB4VS2-01 & $\mathrm{Ce}_{2} \mathrm{O}_{3}$ & 0.0170 & 0.0170 & 0.0224 & -0.0054 & -0.0054 & $-24.0 \%$ & $-24.0 \%$ \\
\hline SB4VS2-01 & $\mathrm{Cr}_{2} \mathrm{O}_{3}$ & 0.0621 & 0.0604 & 0.0558 & 0.0063 & 0.0046 & $11.3 \%$ & $8.2 \%$ \\
\hline SB4VS2-01 & $\mathrm{CuO}$ & 0.0275 & 0.0290 & 0.0199 & 0.0076 & 0.0091 & $38.4 \%$ & $45.6 \%$ \\
\hline SB4VS2-01 & $\mathrm{Fe}_{2} \mathrm{O}_{3}$ & 9.9757 & 10.0071 & 9.8673 & 0.1084 & 0.1398 & $1.1 \%$ & $1.4 \%$ \\
\hline SB4VS2-01 & $\mathrm{La}_{2} \mathrm{O}_{3}$ & 0.0144 & 0.0144 & 0.0187 & -0.0043 & -0.0043 & $-23.2 \%$ & $-23.2 \%$ \\
\hline SB4VS2-01 & $\mathrm{Li}_{2} \mathrm{O}$ & 5.3392 & 5.5150 & 5.4400 & -0.1008 & 0.0750 & $-1.9 \%$ & $1.4 \%$ \\
\hline SB4VS2-01 & $\mathrm{MgO}$ & 0.9324 & 0.9620 & 0.9339 & -0.0015 & 0.0281 & $-0.2 \%$ & $3.0 \%$ \\
\hline SB4VS2-01 & $\mathrm{MnO}$ & 1.9368 & 2.0276 & 1.9803 & -0.0435 & 0.0473 & $-2.2 \%$ & $2.4 \%$ \\
\hline SB4VS2-01 & $\mathrm{Na}_{2} \mathrm{O}$ & 10.1774 & 9.9718 & 10.1966 & -0.0192 & -0.2248 & $-0.2 \%$ & $-2.2 \%$ \\
\hline SB4VS2-01 & $\mathrm{NiO}$ & 0.5084 & 0.5394 & 0.5507 & -0.0423 & -0.0113 & $-7.7 \%$ & $-2.0 \%$ \\
\hline SB4VS2-01 & $\mathrm{PbO}$ & 0.0232 & 0.0232 & 0.0206 & 0.0026 & 0.0026 & $12.4 \%$ & $12.4 \%$ \\
\hline SB4VS2-01 & $\mathrm{SiO}_{2}$ & 48.3482 & 48.8504 & 48.5258 & -0.1776 & 0.3246 & $-0.4 \%$ & $0.7 \%$ \\
\hline SB4VS2-01 & $\mathrm{SO}_{4}$ & 0.1917 & 0.1917 & 0.1863 & 0.0054 & 0.0054 & $2.9 \%$ & $2.9 \%$ \\
\hline SB4VS2-01 & $\mathrm{TiO}_{2}$ & 0.0284 & 0.0296 & 0.0159 & 0.0125 & 0.0137 & $78.3 \%$ & $86.4 \%$ \\
\hline SB4VS2-01 & $\mathrm{U}_{3} \mathrm{O}_{8}$ & 2.8271 & 3.0359 & 3.0093 & -0.1822 & 0.0266 & $-6.1 \%$ & $0.9 \%$ \\
\hline SB4VS2-01 & $\mathrm{ZrO}_{2}$ & 0.0240 & 0.0240 & 0.0258 & -0.0018 & -0.0018 & $-7.1 \%$ & $-7.1 \%$ \\
\hline SB4VS2-01 & Sum & 99.3346 & 100.7277 & 100.0001 & -0.6655 & 0.7276 & $-0.7 \%$ & $0.7 \%$ \\
\hline SB4VS2-02 & $\mathrm{Al}_{2} \mathrm{O}_{3}$ & 9.8774 & 10.0543 & 9.4426 & 0.4348 & 0.6117 & $4.6 \%$ & $6.5 \%$ \\
\hline SB4VS2-02 & $\mathrm{B}_{2} \mathrm{O}_{3}$ & 9.0050 & 9.0118 & 9.1000 & -0.0950 & -0.0882 & $-1.0 \%$ & $-1.0 \%$ \\
\hline SB4VS2-02 & $\mathrm{BaO}$ & 0.0294 & 0.0323 & 0.0272 & 0.0022 & 0.0051 & $8.3 \%$ & $18.7 \%$ \\
\hline SB4VS2-02 & $\mathrm{CaO}$ & 0.9861 & 1.0481 & 1.0421 & -0.0560 & 0.0060 & $-5.4 \%$ & $0.6 \%$ \\
\hline SB4VS2-02 & $\mathrm{Ce}_{2} \mathrm{O}_{3}$ & 0.0183 & 0.0183 & 0.0245 & -0.0062 & -0.0062 & $-25.2 \%$ & $-25.2 \%$ \\
\hline SB4VS2-02 & $\mathrm{Cr}_{2} \mathrm{O}_{3}$ & 0.0541 & 0.0541 & 0.0610 & -0.0069 & -0.0069 & $-11.3 \%$ & $-11.3 \%$ \\
\hline SB4VS2-02 & $\mathrm{CuO}$ & 0.0286 & 0.0306 & 0.0218 & 0.0068 & 0.0088 & $31.4 \%$ & $40.3 \%$ \\
\hline SB4VS2-02 & $\mathrm{Fe}_{2} \mathrm{O}_{3}$ & 10.3892 & 10.5126 & 10.7924 & -0.4032 & -0.2798 & $-3.7 \%$ & $-2.6 \%$ \\
\hline SB4VS2-02 & $\mathrm{La}_{2} \mathrm{O}_{3}$ & 0.0172 & 0.0172 & 0.0204 & -0.0032 & -0.0032 & $-15.9 \%$ & $-15.9 \%$ \\
\hline SB4VS2-02 & $\mathrm{Li}_{2} \mathrm{O}$ & 5.1705 & 5.2900 & 5.2000 & -0.0295 & 0.0900 & $-0.6 \%$ & $1.7 \%$ \\
\hline SB4VS2-02 & $\mathrm{MgO}$ & 1.0431 & 1.1103 & 1.0215 & 0.0216 & 0.0888 & $2.1 \%$ & $8.7 \%$ \\
\hline SB4VS2-02 & $\mathrm{MnO}$ & 2.2273 & 2.2706 & 2.1660 & 0.0613 & 0.1046 & $2.8 \%$ & $4.8 \%$ \\
\hline SB4VS2-02 & $\mathrm{Na}_{2} \mathrm{O}$ & 10.7452 & 10.6081 & 10.4025 & 0.3427 & 0.2056 & $3.3 \%$ & $2.0 \%$ \\
\hline SB4VS2-02 & $\mathrm{NiO}$ & 0.5478 & 0.6007 & 0.6023 & -0.0545 & -0.0016 & $-9.0 \%$ & $-0.3 \%$ \\
\hline SB4VS2-02 & $\mathrm{PbO}$ & 0.0211 & 0.0211 & 0.0225 & -0.0014 & -0.0014 & $-6.0 \%$ & $-6.0 \%$ \\
\hline SB4VS2-02 & $\mathrm{SiO}_{2}$ & 47.5459 & 48.5605 & 46.5126 & 1.0333 & 2.0479 & $2.2 \%$ & $4.4 \%$ \\
\hline SB4VS2-02 & $\mathrm{SO}_{4}$ & 0.2138 & 0.2138 & 0.2038 & 0.0101 & 0.0101 & $4.9 \%$ & $4.9 \%$ \\
\hline SB4VS2-02 & $\mathrm{TiO}_{2}$ & 0.0290 & 0.0312 & 0.0174 & 0.0116 & 0.0138 & $66.6 \%$ & $79.3 \%$ \\
\hline SB4VS2-02 & $\mathrm{U}_{3} \mathrm{O}_{8}$ & 3.1853 & 3.3714 & 3.2914 & -0.1061 & 0.0800 & $-3.2 \%$ & $2.4 \%$ \\
\hline SB4VS2-02 & $\mathrm{ZrO}_{2}$ & 0.0289 & 0.0289 & 0.0282 & 0.0007 & 0.0007 & $2.4 \%$ & $2.4 \%$ \\
\hline SB4VS2-02 & Sum & 101.1633 & 102.8858 & 100.0001 & 1.1631 & 2.8857 & $1.2 \%$ & $2.9 \%$ \\
\hline SB4VS2-03 & $\mathrm{Al}_{2} \mathrm{O}_{3}$ & 10.1088 & 10.4092 & 10.2520 & -0.1432 & 0.1572 & $-1.4 \%$ & $1.5 \%$ \\
\hline SB4VS2-03 & $\mathrm{B}_{2} \mathrm{O}_{3}$ & 8.5166 & 8.7223 & 8.6800 & -0.1634 & 0.0423 & $-1.9 \%$ & $0.5 \%$ \\
\hline SB4VS2-03 & $\mathrm{BaO}$ & 0.0271 & 0.0288 & 0.0295 & -0.0024 & -0.0007 & $-8.2 \%$ & $-2.5 \%$ \\
\hline SB4VS2-03 & $\mathrm{CaO}$ & 1.0802 & 1.1336 & 1.1314 & -0.0512 & 0.0022 & $-4.5 \%$ & $0.2 \%$ \\
\hline SB4VS2-03 & $\mathrm{Ce}_{2} \mathrm{O}_{3}$ & 0.0278 & 0.0278 & 0.0266 & 0.0013 & 0.0013 & $4.8 \%$ & $4.8 \%$ \\
\hline SB4VS2-03 & $\mathrm{Cr}_{2} \mathrm{O}_{3}$ & 0.0636 & 0.0618 & 0.0663 & -0.0027 & -0.0045 & $-4.1 \%$ & $-6.8 \%$ \\
\hline SB4VS2-03 & $\mathrm{CuO}$ & 0.0282 & 0.0296 & 0.0236 & 0.0046 & 0.0060 & $19.3 \%$ & $25.5 \%$ \\
\hline SB4VS2-03 & $\mathrm{Fe}_{2} \mathrm{O}_{3}$ & 11.3768 & 11.4130 & 11.7174 & -0.3406 & -0.3044 & $-2.9 \%$ & $-2.6 \%$ \\
\hline SB4VS2-03 & $\mathrm{La}_{2} \mathrm{O}_{3}$ & 0.0164 & 0.0164 & 0.0222 & -0.0058 & -0.0058 & $-26.0 \%$ & $-26.0 \%$ \\
\hline SB4VS2-03 & $\mathrm{Li}_{2} \mathrm{O}$ & 4.8225 & 4.9813 & 4.9600 & -0.1375 & 0.0213 & $-2.8 \%$ & $0.4 \%$ \\
\hline SB4VS2-03 & $\mathrm{MgO}$ & 1.0783 & 1.1123 & 1.1090 & -0.0307 & 0.0033 & $-2.8 \%$ & $0.3 \%$ \\
\hline SB4VS2-03 & $\mathrm{MnO}$ & 2.2531 & 2.3592 & 2.3516 & -0.0985 & 0.0076 & $-4.2 \%$ & $0.3 \%$ \\
\hline SB4VS2-03 & $\mathrm{Na}_{2} \mathrm{O}$ & 10.6020 & 10.3878 & 10.6084 & -0.0064 & -0.2206 & $-0.1 \%$ & $-2.1 \%$ \\
\hline SB4VS2-03 & $\mathrm{NiO}$ & 0.5500 & 0.5835 & 0.6539 & -0.1039 & -0.0704 & $-15.9 \%$ & $-10.8 \%$ \\
\hline SB4VS2-03 & $\mathrm{PbO}$ & 0.0229 & 0.0229 & 0.0244 & -0.0015 & -0.0015 & $-6.2 \%$ & $-6.2 \%$ \\
\hline SB4VS2-03 & $\mathrm{SiO}_{2}$ & 43.9626 & 44.4198 & 44.4993 & -0.5367 & -0.0795 & $-1.2 \%$ & $-0.2 \%$ \\
\hline
\end{tabular}


WSRC-STI-2008-00315

Revision 0

Table A4. Average Measured and Bias-Corrected Chemical Compositions Versus Targeted Compositions by Oxide by SB4-Decant Variability Study Glass

\begin{tabular}{|c|c|c|c|c|c|c|c|c|}
\hline & & & Measured & & & & & \\
\hline Glass & & Measured & Bias-Corrected & Targeted & Diff of & Diff of & \% Diff of & $\%$ Diff of \\
\hline ID & Oxide & $(\mathrm{wt} \%)$ & $(\mathrm{wt} \%)$ & (wt\%) & Measured & Meas BC & Measured & Meas BC \\
\hline SB4VS2-03 & $\mathrm{SO}_{4}$ & 0.2217 & 0.2217 & 0.2212 & 0.0005 & 0.0005 & $0.2 \%$ & $0.2 \%$ \\
\hline SB4VS2-03 & $\mathrm{TiO}_{2}$ & 0.0313 & 0.0327 & 0.0189 & 0.0124 & 0.0138 & $65.5 \%$ & $72.9 \%$ \\
\hline SB4VS2-03 & $\mathrm{U}_{3} \mathrm{O}_{8}$ & 3.3430 & 3.5900 & 3.5735 & -0.2305 & 0.0165 & $-6.4 \%$ & $0.5 \%$ \\
\hline SB4VS2-03 & $\mathrm{ZrO}_{2}$ & 0.0304 & 0.0304 & 0.0306 & -0.0002 & -0.0002 & $-0.7 \%$ & $-0.7 \%$ \\
\hline SB4VS2-03 & Sum & 98.1634 & 99.5842 & 99.9998 & -1.8364 & -0.4156 & $-1.8 \%$ & $-0.4 \%$ \\
\hline SB4VS2-04 & $\mathrm{Al}_{2} \mathrm{O}_{3}$ & 8.2949 & 8.5413 & 8.3054 & -0.0105 & 0.2359 & $-0.1 \%$ & $2.8 \%$ \\
\hline SB4VS2-04 & $\mathrm{B}_{2} \mathrm{O}_{3}$ & 9.2492 & 9.4724 & 9.5200 & -0.2708 & -0.0476 & $-2.8 \%$ & $-0.5 \%$ \\
\hline SB4VS2-04 & $\mathrm{BaO}$ & 0.0265 & 0.0282 & 0.0250 & 0.0015 & 0.0032 & $6.1 \%$ & $12.7 \%$ \\
\hline SB4VS2-04 & $\mathrm{CaO}$ & 0.8797 & 0.9233 & 0.9214 & -0.0417 & 0.0019 & $-4.5 \%$ & $0.2 \%$ \\
\hline SB4VS2-04 & $\mathrm{Ce}_{2} \mathrm{O}_{3}$ & 0.0146 & 0.0146 & 0.0239 & -0.0092 & -0.0092 & $-38.6 \%$ & $-38.6 \%$ \\
\hline SB4VS2-04 & $\mathrm{Cr}_{2} \mathrm{O}_{3}$ & 0.0599 & 0.0583 & 0.0547 & 0.0052 & 0.0036 & $9.6 \%$ & $6.5 \%$ \\
\hline SB4VS2-04 & $\mathrm{CuO}$ & 0.0250 & 0.0263 & 0.0196 & 0.0054 & 0.0067 & $27.7 \%$ & $34.4 \%$ \\
\hline SB4VS2-04 & $\mathrm{Fe}_{2} \mathrm{O}_{3}$ & 9.2680 & 9.2971 & 9.5727 & -0.3047 & -0.2756 & $-3.2 \%$ & $-2.9 \%$ \\
\hline SB4VS2-04 & $\mathrm{La}_{2} \mathrm{O}_{3}$ & 0.0158 & 0.0158 & 0.0188 & -0.0030 & -0.0030 & $-15.8 \%$ & $-15.8 \%$ \\
\hline SB4VS2-04 & $\mathrm{Li}_{2} \mathrm{O}$ & 5.3015 & 5.4762 & 5.4400 & -0.1385 & 0.0362 & $-2.5 \%$ & $0.7 \%$ \\
\hline SB4VS2-04 & $\mathrm{MgO}$ & 0.8893 & 0.9175 & 0.8895 & -0.0002 & 0.0280 & $0.0 \%$ & $3.1 \%$ \\
\hline SB4VS2-04 & $\mathrm{MnO}$ & 1.8948 & 1.9834 & 1.9547 & -0.0599 & 0.0287 & $-3.1 \%$ & $1.5 \%$ \\
\hline SB4VS2-04 & $\mathrm{Na}_{2} \mathrm{O}$ & 10.6526 & 10.4374 & 10.5957 & 0.0569 & -0.1583 & $0.5 \%$ & $-1.5 \%$ \\
\hline SB4VS2-04 & $\mathrm{NiO}$ & 0.5100 & 0.5411 & 0.5450 & -0.0350 & -0.0039 & $-6.4 \%$ & $-0.7 \%$ \\
\hline SB4VS2-04 & $\mathrm{PbO}$ & 0.0202 & 0.0202 & 0.0215 & -0.0013 & -0.0013 & $-6.1 \%$ & $-6.1 \%$ \\
\hline SB4VS2-04 & $\mathrm{SiO}_{2}$ & 48.0273 & 48.5262 & 48.4908 & -0.4635 & 0.0354 & $-1.0 \%$ & $0.1 \%$ \\
\hline SB4VS2-04 & $\mathrm{SO}_{4}$ & 0.2165 & 0.2165 & 0.2228 & -0.0063 & -0.0063 & $-2.8 \%$ & $-2.8 \%$ \\
\hline SB4VS2-04 & $\mathrm{TiO}_{2}$ & 0.4287 & 0.4481 & 0.4308 & -0.0021 & 0.0173 & $-0.5 \%$ & $4.0 \%$ \\
\hline SB4VS2-04 & $\mathrm{U}_{3} \mathrm{O}_{8}$ & 2.7564 & 2.9599 & 2.9118 & -0.1554 & 0.0481 & $-5.3 \%$ & $1.7 \%$ \\
\hline SB4VS2-04 & $\mathrm{ZrO}_{2}$ & 0.0294 & 0.0294 & 0.0277 & 0.0017 & 0.0017 & $6.1 \%$ & $6.1 \%$ \\
\hline SB4VS2-04 & Sum & 98.5603 & 99.9332 & 99.9917 & -1.4314 & -0.0585 & $-1.4 \%$ & $-0.1 \%$ \\
\hline SB4VS2-05 & $\mathrm{Al}_{2} \mathrm{O}_{3}$ & 9.1594 & 9.4316 & 9.0841 & 0.0753 & 0.3475 & $0.8 \%$ & $3.8 \%$ \\
\hline SB4VS2-05 & $\mathrm{B}_{2} \mathrm{O}_{3}$ & 9.0238 & 9.2418 & 9.1000 & -0.0762 & 0.1418 & $-0.8 \%$ & $1.6 \%$ \\
\hline SB4VS2-05 & $\mathrm{BaO}$ & 0.0257 & 0.0273 & 0.0273 & -0.0016 & 0.0000 & $-5.9 \%$ & $-0.1 \%$ \\
\hline SB4VS2-05 & $\mathrm{CaO}$ & 0.9724 & 1.0206 & 1.0078 & -0.0354 & 0.0128 & $-3.5 \%$ & $1.3 \%$ \\
\hline SB4VS2-05 & $\mathrm{Ce}_{2} \mathrm{O}_{3}$ & 0.0252 & 0.0252 & 0.0261 & -0.0009 & -0.0009 & $-3.5 \%$ & $-3.5 \%$ \\
\hline SB4VS2-05 & $\mathrm{Cr}_{2} \mathrm{O}_{3}$ & 0.0588 & 0.0572 & 0.0598 & -0.0010 & -0.0026 & $-1.6 \%$ & $-4.4 \%$ \\
\hline SB4VS2-05 & $\mathrm{CuO}$ & 0.0269 & 0.0283 & 0.0215 & 0.0054 & 0.0068 & $25.2 \%$ & $31.7 \%$ \\
\hline SB4VS2-05 & $\mathrm{Fe}_{2} \mathrm{O}_{3}$ & 10.5905 & 10.6247 & 10.4702 & 0.1203 & 0.1545 & $1.1 \%$ & $1.5 \%$ \\
\hline SB4VS2-05 & $\mathrm{La}_{2} \mathrm{O}_{3}$ & 0.0152 & 0.0152 & 0.0206 & -0.0054 & -0.0054 & $-26.0 \%$ & $-26.0 \%$ \\
\hline SB4VS2-05 & $\mathrm{Li}_{2} \mathrm{O}$ & 5.1400 & 5.3096 & 5.2000 & -0.0600 & 0.1096 & $-1.2 \%$ & $2.1 \%$ \\
\hline SB4VS2-05 & $\mathrm{MgO}$ & 0.9668 & 0.9972 & 0.9729 & -0.0061 & 0.0243 & $-0.6 \%$ & $2.5 \%$ \\
\hline SB4VS2-05 & $\mathrm{MnO}$ & 2.1208 & 2.2212 & 2.1380 & -0.0172 & 0.0832 & $-0.8 \%$ & $3.9 \%$ \\
\hline SB4VS2-05 & $\mathrm{Na}_{2} \mathrm{O}$ & 10.9222 & 10.7016 & 10.8390 & 0.0832 & -0.1374 & $0.8 \%$ & $-1.3 \%$ \\
\hline SB4VS2-05 & $\mathrm{NiO}$ & 0.5513 & 0.5848 & 0.5961 & -0.0448 & -0.0113 & $-7.5 \%$ & $-1.9 \%$ \\
\hline SB4VS2-05 & $\mathrm{PbO}$ & 0.0213 & 0.0213 & 0.0235 & -0.0022 & -0.0022 & $-9.5 \%$ & $-9.5 \%$ \\
\hline SB4VS2-05 & $\mathrm{SiO}_{2}$ & 46.5298 & 47.0153 & 46.4743 & 0.0555 & 0.5410 & $0.1 \%$ & $1.2 \%$ \\
\hline SB4VS2-05 & $\mathrm{SO}_{4}$ & 0.2412 & 0.2412 & 0.2436 & -0.0025 & -0.0025 & $-1.0 \%$ & $-1.0 \%$ \\
\hline SB4VS2-05 & $\mathrm{TiO}_{2}$ & 0.4566 & 0.4772 & 0.4711 & -0.0145 & 0.0061 & $-3.1 \%$ & $1.3 \%$ \\
\hline SB4VS2-05 & $\mathrm{U}_{3} \mathrm{O}_{8}$ & 2.9981 & 3.2196 & 3.1848 & -0.1867 & 0.0348 & $-5.9 \%$ & $1.1 \%$ \\
\hline SB4VS2-05 & $\mathrm{ZrO}_{2}$ & 0.0267 & 0.0267 & 0.0303 & -0.0036 & -0.0036 & $-12.0 \%$ & $-12.0 \%$ \\
\hline SB4VS2-05 & Sum & 99.8727 & 101.2875 & 99.9910 & -0.1184 & 1.2964 & $-0.1 \%$ & $1.3 \%$ \\
\hline SB4VS2-06 & $\mathrm{Al}_{2} \mathrm{O}_{3}$ & 9.8915 & 10.1854 & 9.8627 & 0.0288 & 0.3227 & $0.3 \%$ & $3.3 \%$ \\
\hline SB4VS2-06 & $\mathrm{B}_{2} \mathrm{O}_{3}$ & 8.4603 & 8.6647 & 8.6800 & -0.2197 & -0.0153 & $-2.5 \%$ & $-0.2 \%$ \\
\hline SB4VS2-06 & $\mathrm{BaO}$ & 0.0251 & 0.0267 & 0.0297 & -0.0046 & -0.0030 & $-15.4 \%$ & $-10.2 \%$ \\
\hline SB4VS2-06 & $\mathrm{CaO}$ & 1.0414 & 1.0929 & 1.0942 & -0.0528 & -0.0013 & $-4.8 \%$ & $-0.1 \%$ \\
\hline SB4VS2-06 & $\mathrm{Ce}_{2} \mathrm{O}_{3}$ & 0.0231 & 0.0231 & 0.0283 & -0.0052 & -0.0052 & $-18.3 \%$ & $-18.3 \%$ \\
\hline SB4VS2-06 & $\mathrm{Cr}_{2} \mathrm{O}_{3}$ & 0.0607 & 0.0589 & 0.0650 & -0.0043 & -0.0061 & $-6.7 \%$ & $-9.3 \%$ \\
\hline SB4VS2-06 & $\mathrm{CuO}$ & 0.0282 & 0.0296 & 0.0233 & 0.0049 & 0.0063 & $20.9 \%$ & $27.2 \%$ \\
\hline SB4VS2-06 & $\mathrm{Fe}_{2} \mathrm{O}_{3}$ & 10.8872 & 10.9221 & 11.3676 & -0.4804 & -0.4455 & $-4.2 \%$ & $-3.9 \%$ \\
\hline SB4VS2-06 & $\mathrm{La}_{2} \mathrm{O}_{3}$ & 0.0150 & 0.0150 & 0.0223 & -0.0073 & -0.0073 & $-32.9 \%$ & $-32.9 \%$ \\
\hline SB4VS2-06 & $\mathrm{Li}_{2} \mathrm{O}$ & 4.7579 & 4.9145 & 4.9600 & -0.2021 & -0.0455 & $-4.1 \%$ & $-0.9 \%$ \\
\hline SB4VS2-06 & $\mathrm{MgO}$ & 1.0410 & 1.0739 & 1.0563 & -0.0153 & 0.0176 & $-1.4 \%$ & $1.7 \%$ \\
\hline
\end{tabular}


WSRC-STI-2008-00315

Revision 0

Table A4. Average Measured and Bias-Corrected Chemical Compositions Versus Targeted Compositions by Oxide by SB4-Decant Variability Study Glass

\begin{tabular}{|c|c|c|c|c|c|c|c|c|}
\hline & & & Measured & & & & & \\
\hline Glass & & Measured & Bias-Corrected & Targeted & Diff of & Diff of & \% Diff of & $\%$ Diff of \\
\hline ID & Oxide & (wt\%) & $(\mathrm{wt} \%)$ & (wt\%) & Measured & Meas BC & Measured & Meas BC \\
\hline SB4VS2-06 & $\mathrm{MnO}$ & 2.2887 & 2.3962 & 2.3212 & -0.0325 & 0.0750 & $-1.4 \%$ & $3.2 \%$ \\
\hline SB4VS2-06 & $\mathrm{Na}_{2} \mathrm{O}$ & 11.0974 & 10.8732 & 11.0824 & 0.0150 & -0.2092 & $0.1 \%$ & $-1.9 \%$ \\
\hline SB4VS2-06 & $\mathrm{NiO}$ & 0.5857 & 0.6213 & 0.6472 & -0.0615 & -0.0259 & $-9.5 \%$ & $-4.0 \%$ \\
\hline SB4VS2-06 & $\mathrm{PbO}$ & 0.0315 & 0.0315 & 0.0255 & 0.0060 & 0.0060 & $23.6 \%$ & $23.6 \%$ \\
\hline SB4VS2-06 & $\mathrm{SiO}_{2}$ & 44.6579 & 45.1227 & 44.4579 & 0.2000 & 0.6648 & $0.4 \%$ & $1.5 \%$ \\
\hline SB4VS2-06 & $\mathrm{SO}_{4}$ & 0.2681 & 0.2681 & 0.2645 & 0.0036 & 0.0036 & $1.4 \%$ & $1.4 \%$ \\
\hline SB4VS2-06 & $\mathrm{TiO}_{2}$ & 0.4983 & 0.5209 & 0.5115 & -0.0132 & 0.0094 & $-2.6 \%$ & $1.8 \%$ \\
\hline SB4VS2-06 & $\mathrm{U}_{3} \mathrm{O}_{8}$ & 3.2841 & 3.5266 & 3.4577 & -0.1736 & 0.0689 & $-5.0 \%$ & $2.0 \%$ \\
\hline SB4VS2-06 & $\mathrm{ZrO}_{2}$ & 0.0280 & 0.0280 & 0.0329 & -0.0049 & -0.0049 & $-14.8 \%$ & $-14.8 \%$ \\
\hline SB4VS2-06 & Sum & 98.9710 & 100.3955 & 99.9903 & -1.0193 & 0.4052 & $-1.0 \%$ & $0.4 \%$ \\
\hline SB4VS2-07 & $\mathrm{Al}_{2} \mathrm{O}_{3}$ & 8.1201 & 8.3613 & 8.1090 & 0.0111 & 0.2523 & $0.1 \%$ & $3.1 \%$ \\
\hline SB4VS2-07 & $\mathrm{B}_{2} \mathrm{O}_{3}$ & 9.3055 & 9.5303 & 9.5200 & -0.2145 & 0.0103 & $-2.3 \%$ & $0.1 \%$ \\
\hline SB4VS2-07 & $\mathrm{BaO}$ & 0.0148 & 0.0157 & 0.0244 & -0.0096 & -0.0087 & $-39.4 \%$ & $-35.6 \%$ \\
\hline SB4VS2-07 & $\mathrm{CaO}$ & 0.8766 & 0.9200 & 0.8996 & -0.0230 & 0.0204 & $-2.6 \%$ & $2.3 \%$ \\
\hline SB4VS2-07 & $\mathrm{Ce}_{2} \mathrm{O}_{3}$ & 0.0196 & 0.0196 & 0.0233 & -0.0037 & -0.0037 & $-15.8 \%$ & $-15.8 \%$ \\
\hline SB4VS2-07 & $\mathrm{Cr}_{2} \mathrm{O}_{3}$ & 0.0581 & 0.0565 & 0.0534 & 0.0047 & 0.0031 & $8.8 \%$ & $5.7 \%$ \\
\hline SB4VS2-07 & $\mathrm{CuO}$ & 0.0269 & 0.0283 & 0.0192 & 0.0077 & 0.0091 & $40.2 \%$ & $47.5 \%$ \\
\hline SB4VS2-07 & $\mathrm{Fe}_{2} \mathrm{O}_{3}$ & 9.2108 & 9.2407 & 9.3463 & -0.1355 & -0.1056 & $-1.4 \%$ & $-1.1 \%$ \\
\hline SB4VS2-07 & $\mathrm{La}_{2} \mathrm{O}_{3}$ & 0.0167 & 0.0167 & 0.0184 & -0.0017 & -0.0017 & $-9.2 \%$ & $-9.2 \%$ \\
\hline SB4VS2-07 & $\mathrm{Li}_{2} \mathrm{O}$ & 5.2746 & 5.4482 & 5.4400 & -0.1654 & 0.0082 & $-3.0 \%$ & $0.2 \%$ \\
\hline SB4VS2-07 & $\mathrm{MgO}$ & 0.8719 & 0.8994 & 0.8684 & 0.0035 & 0.0310 & $0.4 \%$ & $3.6 \%$ \\
\hline SB4VS2-07 & $\mathrm{MnO}$ & 1.8593 & 1.9468 & 1.9085 & -0.0492 & 0.0383 & $-2.6 \%$ & $2.0 \%$ \\
\hline SB4VS2-07 & $\mathrm{Na}_{2} \mathrm{O}$ & 11.1749 & 10.9492 & 11.2357 & -0.0608 & -0.2865 & $-0.5 \%$ & $-2.5 \%$ \\
\hline SB4VS2-07 & $\mathrm{NiO}$ & 0.4902 & 0.5201 & 0.5322 & -0.0420 & -0.0121 & $-7.9 \%$ & $-2.3 \%$ \\
\hline SB4VS2-07 & $\mathrm{PbO}$ & 0.0207 & 0.0207 & 0.0210 & -0.0003 & -0.0003 & $-1.3 \%$ & $-1.3 \%$ \\
\hline SB4VS2-07 & $\mathrm{SiO}_{2}$ & 48.0273 & 48.5277 & 48.4698 & -0.4425 & 0.0579 & $-0.9 \%$ & $0.1 \%$ \\
\hline SB4VS2-07 & $\mathrm{SO}_{4}$ & 0.2202 & 0.2202 & 0.2175 & 0.0027 & 0.0027 & $1.2 \%$ & $1.2 \%$ \\
\hline SB4VS2-07 & $\mathrm{TiO}_{2}$ & 0.4245 & 0.4437 & 0.4206 & 0.0039 & 0.0231 & $0.9 \%$ & $5.5 \%$ \\
\hline SB4VS2-07 & $\mathrm{U}_{3} \mathrm{O}_{8}$ & 2.6591 & 2.8555 & 2.8429 & -0.1838 & 0.0126 & $-6.5 \%$ & $0.4 \%$ \\
\hline SB4VS2-07 & $\mathrm{ZrO}_{2}$ & 0.0287 & 0.0287 & 0.0270 & 0.0017 & 0.0017 & $6.3 \%$ & $6.3 \%$ \\
\hline SB4VS2-07 & Sum & 98.7007 & 100.0494 & 99.9972 & -1.2965 & 0.0522 & $-1.3 \%$ & $0.1 \%$ \\
\hline SB4VS2-08 & $\mathrm{Al}_{2} \mathrm{O}_{3}$ & 8.9184 & 9.1834 & 8.8692 & 0.0492 & 0.3142 & $0.6 \%$ & $3.5 \%$ \\
\hline SB4VS2-08 & $\mathrm{B}_{2} \mathrm{O}_{3}$ & 8.8064 & 9.0193 & 9.1000 & -0.2936 & -0.0807 & $-3.2 \%$ & $-0.9 \%$ \\
\hline SB4VS2-08 & $\mathrm{BaO}$ & 0.0276 & 0.0293 & 0.0267 & 0.0009 & 0.0026 & $3.5 \%$ & $9.9 \%$ \\
\hline SB4VS2-08 & $\mathrm{CaO}$ & 0.9347 & 0.9809 & 0.9839 & -0.0492 & -0.0030 & $-5.0 \%$ & $-0.3 \%$ \\
\hline SB4VS2-08 & $\mathrm{Ce}_{2} \mathrm{O}_{3}$ & 0.0258 & 0.0258 & 0.0255 & 0.0003 & 0.0003 & $1.2 \%$ & $1.2 \%$ \\
\hline SB4VS2-08 & $\mathrm{Cr}_{2} \mathrm{O}_{3}$ & 0.0563 & 0.0547 & 0.0584 & -0.0021 & -0.0037 & $-3.6 \%$ & $-6.4 \%$ \\
\hline SB4VS2-08 & $\mathrm{CuO}$ & 0.0266 & 0.0280 & 0.0210 & 0.0056 & 0.0070 & $26.7 \%$ & $33.2 \%$ \\
\hline SB4VS2-08 & $\mathrm{Fe}_{2} \mathrm{O}_{3}$ & 9.6576 & 9.6891 & 10.2225 & -0.5649 & -0.5334 & $-5.5 \%$ & $-5.2 \%$ \\
\hline SB4VS2-08 & $\mathrm{La}_{2} \mathrm{O}_{3}$ & 0.0155 & 0.0155 & 0.0201 & -0.0046 & -0.0046 & $-22.7 \%$ & $-22.7 \%$ \\
\hline SB4VS2-08 & $\mathrm{Li}_{2} \mathrm{O}$ & 5.0216 & 5.1870 & 5.2000 & -0.1784 & -0.0130 & $-3.4 \%$ & $-0.2 \%$ \\
\hline SB4VS2-08 & $\mathrm{MgO}$ & 0.9626 & 0.9931 & 0.9499 & 0.0127 & 0.0432 & $1.3 \%$ & $4.5 \%$ \\
\hline SB4VS2-08 & $\mathrm{MnO}$ & 2.0756 & 2.1734 & 2.0874 & -0.0118 & 0.0860 & $-0.6 \%$ & $4.1 \%$ \\
\hline SB4VS2-08 & $\mathrm{Na}_{2} \mathrm{O}$ & 11.6231 & 11.3881 & 11.5390 & 0.0841 & -0.1509 & $0.7 \%$ & $-1.3 \%$ \\
\hline SB4VS2-08 & $\mathrm{NiO}$ & 0.5456 & 0.5788 & 0.5820 & -0.0364 & -0.0032 & $-6.3 \%$ & $-0.6 \%$ \\
\hline SB4VS2-08 & $\mathrm{PbO}$ & 0.0242 & 0.0242 & 0.0229 & 0.0013 & 0.0013 & $5.8 \%$ & $5.8 \%$ \\
\hline SB4VS2-08 & $\mathrm{SiO}_{2}$ & 46.4228 & 46.9057 & 46.4513 & -0.0285 & 0.4544 & $-0.1 \%$ & $1.0 \%$ \\
\hline SB4VS2-08 & $\mathrm{SO}_{4}$ & 0.2554 & 0.2554 & 0.2379 & 0.0175 & 0.0175 & $7.4 \%$ & $7.4 \%$ \\
\hline SB4VS2-08 & $\mathrm{TiO}_{2}$ & 0.4629 & 0.4838 & 0.4600 & 0.0029 & 0.0238 & $0.6 \%$ & $5.2 \%$ \\
\hline SB4VS2-08 & $\mathrm{U}_{3} \mathrm{O}_{8}$ & 2.9303 & 3.1468 & 3.1094 & -0.1791 & 0.0374 & $-5.8 \%$ & $1.2 \%$ \\
\hline SB4VS2-08 & $\mathrm{ZrO}_{2}$ & 0.0250 & 0.0250 & 0.0296 & -0.0046 & -0.0046 & $-15.6 \%$ & $-15.6 \%$ \\
\hline SB4VS2-08 & Sum & 98.8182 & 100.1872 & 99.9967 & -1.1785 & 0.1905 & $-1.2 \%$ & $0.2 \%$ \\
\hline SB4VS2-09 & $\mathrm{Al}_{2} \mathrm{O}_{3}$ & 9.9648 & 10.1430 & 9.6294 & 0.3354 & 0.5136 & $3.5 \%$ & $5.3 \%$ \\
\hline SB4VS2-09 & $\mathrm{B}_{2} \mathrm{O}_{3}$ & 8.5368 & 8.5944 & 8.6800 & -0.1432 & -0.0856 & $-1.7 \%$ & $-1.0 \%$ \\
\hline SB4VS2-09 & $\mathrm{BaO}$ & 0.0264 & 0.0289 & 0.0290 & -0.0026 & -0.0001 & $-9.0 \%$ & $-0.2 \%$ \\
\hline SB4VS2-09 & $\mathrm{CaO}$ & 1.0230 & 1.0873 & 1.0683 & -0.0453 & 0.0190 & $-4.2 \%$ & $1.8 \%$ \\
\hline SB4VS2-09 & $\mathrm{Ce}_{2} \mathrm{O}_{3}$ & 0.0269 & 0.0269 & 0.0277 & -0.0007 & -0.0007 & $-2.6 \%$ & $-2.6 \%$ \\
\hline SB4VS2-09 & $\mathrm{Cr}_{2} \mathrm{O}_{3}$ & 0.0565 & 0.0565 & 0.0634 & -0.0069 & -0.0069 & $-11.0 \%$ & $-10.9 \%$ \\
\hline
\end{tabular}


WSRC-STI-2008-00315

Revision 0

Table A4. Average Measured and Bias-Corrected Chemical Compositions Versus Targeted Compositions by Oxide by SB4-Decant Variability Study Glass

\begin{tabular}{|c|c|c|c|c|c|c|c|c|}
\hline & & & Measured & & & & & \\
\hline Glass & & Measured & Bias-Corrected & Targeted & Diff of & Diff of & \% Diff of & $\%$ Diff of \\
\hline ID & Oxide & $(\mathrm{wt} \%)$ & $(\mathrm{wt} \%)$ & $(\mathrm{wt} \%)$ & Measured & Meas BC & Measured & Meas BC \\
\hline SB4VS2-09 & $\mathrm{CuO}$ & 0.0260 & 0.0277 & 0.0228 & 0.0032 & 0.0049 & $13.9 \%$ & $21.7 \%$ \\
\hline SB4VS2-09 & $\mathrm{Fe}_{2} \mathrm{O}_{3}$ & 10.4332 & 10.5346 & 11.0988 & -0.6656 & -0.5642 & $-6.0 \%$ & $-5.1 \%$ \\
\hline SB4VS2-09 & $\mathrm{La}_{2} \mathrm{O}_{3}$ & 0.0172 & 0.0172 & 0.0218 & -0.0046 & -0.0046 & $-21.3 \%$ & $-21.3 \%$ \\
\hline SB4VS2-09 & $\mathrm{Li}_{2} \mathrm{O}$ & 4.9167 & 5.0427 & 4.9600 & -0.0433 & 0.0827 & $-0.9 \%$ & $1.7 \%$ \\
\hline SB4VS2-09 & $\mathrm{MgO}$ & 1.0163 & 1.0823 & 1.0313 & -0.0150 & 0.0510 & $-1.5 \%$ & $4.9 \%$ \\
\hline SB4VS2-09 & $\mathrm{MnO}$ & 2.3032 & 2.3487 & 2.2663 & 0.0369 & 0.0824 & $1.6 \%$ & $3.6 \%$ \\
\hline SB4VS2-09 & $\mathrm{Na}_{2} \mathrm{O}$ & 12.0225 & 11.8691 & 11.8424 & 0.1801 & 0.0267 & $1.5 \%$ & $0.2 \%$ \\
\hline SB4VS2-09 & $\mathrm{NiO}$ & 0.5857 & 0.6425 & 0.6319 & -0.0462 & 0.0106 & $-7.3 \%$ & $1.7 \%$ \\
\hline SB4VS2-09 & $\mathrm{PbO}$ & 0.0241 & 0.0241 & 0.0249 & -0.0008 & -0.0008 & $-3.2 \%$ & $-3.2 \%$ \\
\hline SB4VS2-09 & $\mathrm{SiO}_{2}$ & 45.1125 & 46.0686 & 44.4328 & 0.6797 & 1.6358 & $1.5 \%$ & $3.7 \%$ \\
\hline SB4VS2-09 & $\mathrm{SO}_{4}$ & 0.2696 & 0.2696 & 0.2583 & 0.0114 & 0.0114 & $4.4 \%$ & $4.4 \%$ \\
\hline SB4VS2-09 & $\mathrm{TiO}_{2}$ & 0.4545 & 0.4894 & 0.4994 & -0.0449 & -0.0100 & $-9.0 \%$ & $-2.0 \%$ \\
\hline SB4VS2-09 & $\mathrm{U}_{3} \mathrm{O}_{8}$ & 3.2693 & 3.4608 & 3.3759 & -0.1066 & 0.0849 & $-3.2 \%$ & $2.5 \%$ \\
\hline SB4VS2-09 & $\mathrm{ZrO}_{2}$ & 0.0314 & 0.0314 & 0.0321 & -0.0007 & -0.0007 & $-2.2 \%$ & $-2.2 \%$ \\
\hline SB4VS2-09 & Sum & 100.1165 & 101.8458 & 99.9964 & 0.1200 & 1.8494 & $0.1 \%$ & $1.8 \%$ \\
\hline Batch 1 & $\mathrm{Al}_{2} \mathrm{O}_{3}$ & 4.7639 & 4.8770 & 4.8770 & -0.1131 & 0.0000 & $-2.3 \%$ & $0.0 \%$ \\
\hline Batch 1 & $\mathrm{~B}_{2} \mathrm{O}_{3}$ & 7.6593 & 7.7770 & 7.7770 & -0.1177 & 0.0000 & $-1.5 \%$ & $0.0 \%$ \\
\hline Batch 1 & $\mathrm{BaO}$ & 0.1400 & 0.1510 & 0.1510 & -0.0110 & 0.0000 & $-7.3 \%$ & $0.0 \%$ \\
\hline Batch 1 & $\mathrm{CaO}$ & 1.1552 & 1.2200 & 1.2200 & -0.0648 & 0.0000 & $-5.3 \%$ & $0.0 \%$ \\
\hline Batch 1 & $\mathrm{Ce}_{2} \mathrm{O}_{3}$ & 0.0059 & 0.0059 & 0.0000 & 0.0059 & 0.0059 & & \\
\hline Batch 1 & $\mathrm{Cr}_{2} \mathrm{O}_{3}$ & 0.1085 & 0.1070 & 0.1070 & 0.0015 & 0.0000 & $1.4 \%$ & $0.0 \%$ \\
\hline Batch 1 & $\mathrm{CuO}$ & 0.3766 & 0.3990 & 0.3990 & -0.0224 & 0.0000 & $-5.6 \%$ & $0.0 \%$ \\
\hline Batch 1 & $\mathrm{Fe}_{2} \mathrm{O}_{3}$ & 12.7583 & 12.8390 & 12.8390 & -0.0807 & 0.0000 & $-0.6 \%$ & $0.0 \%$ \\
\hline Batch 1 & $\mathrm{La}_{2} \mathrm{O}_{3}$ & 0.0059 & 0.0059 & 0.0000 & 0.0059 & 0.0059 & & \\
\hline Batch 1 & $\mathrm{Li}_{2} \mathrm{O}$ & 4.3031 & 4.4290 & 4.4290 & -0.1259 & 0.0000 & $-2.8 \%$ & $0.0 \%$ \\
\hline Batch 1 & $\mathrm{MgO}$ & 1.3544 & 1.4190 & 1.4190 & -0.0646 & 0.0000 & $-4.6 \%$ & $0.0 \%$ \\
\hline Batch 1 & $\mathrm{MnO}$ & 1.6705 & 1.7260 & 1.7260 & -0.0555 & 0.0000 & $-3.2 \%$ & $0.0 \%$ \\
\hline Batch 1 & $\mathrm{Na}_{2} \mathrm{O}$ & 9.1546 & 9.0030 & 9.0030 & 0.1516 & 0.0000 & $1.7 \%$ & $0.0 \%$ \\
\hline Batch 1 & $\mathrm{NiO}$ & 0.6965 & 0.7510 & 0.7510 & -0.0545 & 0.0000 & $-7.3 \%$ & $0.0 \%$ \\
\hline Batch 1 & $\mathrm{PbO}$ & 0.0054 & 0.0054 & 0.0000 & 0.0054 & 0.0054 & & \\
\hline Batch 1 & $\mathrm{SiO}_{2}$ & 49.4446 & 50.2200 & 50.2200 & -0.7754 & 0.0000 & $-1.5 \%$ & $0.0 \%$ \\
\hline Batch 1 & $\mathrm{SO}_{4}$ & 0.0423 & 0.0423 & 0.0000 & 0.0423 & 0.0423 & & \\
\hline Batch 1 & $\mathrm{TiO}_{2}$ & 0.6384 & 0.6770 & 0.6770 & -0.0386 & 0.0000 & $-5.7 \%$ & $0.0 \%$ \\
\hline Batch 1 & $\mathrm{U}_{3} \mathrm{O}_{8}$ & 0.0590 & 0.0629 & 0.0000 & 0.0590 & 0.0629 & & \\
\hline Batch 1 & $\mathrm{ZrO}_{2}$ & 0.0834 & 0.0834 & 0.0980 & -0.0146 & -0.0146 & $-14.9 \%$ & $-14.9 \%$ \\
\hline Batch 1 & Sum & 94.4258 & 95.8007 & 95.6930 & -1.2672 & 0.1077 & $-1.3 \%$ & $0.1 \%$ \\
\hline Ustd & $\mathrm{Al}_{2} \mathrm{O}_{3}$ & 3.9727 & 4.0672 & 4.1000 & -0.1273 & -0.0328 & $-3.1 \%$ & $-0.8 \%$ \\
\hline Ustd & $\mathrm{B}_{2} \mathrm{O}_{3}$ & 9.1445 & 9.0519 & 9.2090 & -0.0645 & -0.1571 & $-0.7 \%$ & $-1.7 \%$ \\
\hline Ustd & $\mathrm{BaO}$ & 0.0056 & 0.0060 & 0.0000 & 0.0056 & 0.0060 & & \\
\hline Ustd & $\mathrm{CaO}$ & 1.2574 & 1.3279 & 1.3010 & -0.0436 & 0.0269 & $-3.4 \%$ & $2.1 \%$ \\
\hline Ustd & $\mathrm{Ce}_{2} \mathrm{O}_{3}$ & 0.0059 & 0.0059 & 0.0000 & 0.0059 & 0.0059 & & \\
\hline Ustd & $\mathrm{Cr}_{2} \mathrm{O}_{3}$ & 0.2413 & 0.2381 & 0.0000 & 0.2413 & 0.2381 & & \\
\hline Ustd & $\mathrm{CuO}$ & 0.0077 & 0.0081 & 0.0000 & 0.0077 & 0.0081 & & \\
\hline Ustd & $\mathrm{Fe}_{2} \mathrm{O}_{3}$ & 12.8316 & 13.0351 & 13.1960 & -0.3644 & -0.1609 & $-2.8 \%$ & $-1.2 \%$ \\
\hline Ustd & $\mathrm{La}_{2} \mathrm{O}_{3}$ & 0.0059 & 0.0059 & 0.0000 & 0.0059 & 0.0059 & & \\
\hline Ustd & $\mathrm{Li}_{2} \mathrm{O}$ & 2.9818 & 3.0367 & 3.0570 & -0.0752 & -0.0203 & $-2.5 \%$ & $-0.7 \%$ \\
\hline Ustd & $\mathrm{MgO}$ & 1.1533 & 1.2089 & 1.2100 & -0.0567 & -0.0011 & $-4.7 \%$ & $-0.1 \%$ \\
\hline Ustd & $\mathrm{MnO}$ & 2.7390 & 2.8305 & 2.8920 & -0.1530 & -0.0615 & $-5.3 \%$ & $-2.1 \%$ \\
\hline Ustd & $\mathrm{Na}_{2} \mathrm{O}$ & 11.8793 & 11.6834 & 11.7950 & 0.0843 & -0.1116 & $0.7 \%$ & $-0.9 \%$ \\
\hline Ustd & $\mathrm{NiO}$ & 0.9984 & 1.0773 & 1.1200 & -0.1216 & -0.0427 & $-10.9 \%$ & $-3.8 \%$ \\
\hline Ustd & $\mathrm{PbO}$ & 0.0054 & 0.0054 & 0.0000 & 0.0054 & 0.0054 & & \\
\hline Ustd & $\mathrm{SiO}_{2}$ & 45.0590 & 45.7691 & 45.3530 & -0.2940 & 0.4161 & $-0.6 \%$ & $0.9 \%$ \\
\hline Ustd & $\mathrm{SO}_{4}$ & 0.0397 & 0.0397 & 0.0000 & 0.0397 & 0.0397 & & \\
\hline Ustd & $\mathrm{TiO}_{2}$ & 0.9257 & 0.9822 & 1.0490 & -0.1233 & -0.0668 & $-11.8 \%$ & $-6.4 \%$ \\
\hline Ustd & $\mathrm{U}_{3} \mathrm{O}_{8}$ & 2.2567 & 2.4060 & 2.4060 & -0.1493 & 0.0000 & $-6.2 \%$ & $0.0 \%$ \\
\hline Ustd & $\mathrm{ZrO}_{2}$ & 0.0068 & 0.0068 & 0.0000 & 0.0068 & 0.0068 & & \\
\hline Ustd & Sum & 95.5175 & 96.7919 & 96.6880 & -1.1705 & 0.1039 & $-1.2 \%$ & $0.1 \%$ \\
\hline
\end{tabular}




\section{Figure A1. Oxide Measurements in Analytical Sequence for Samples Prepared Using the LM Method}

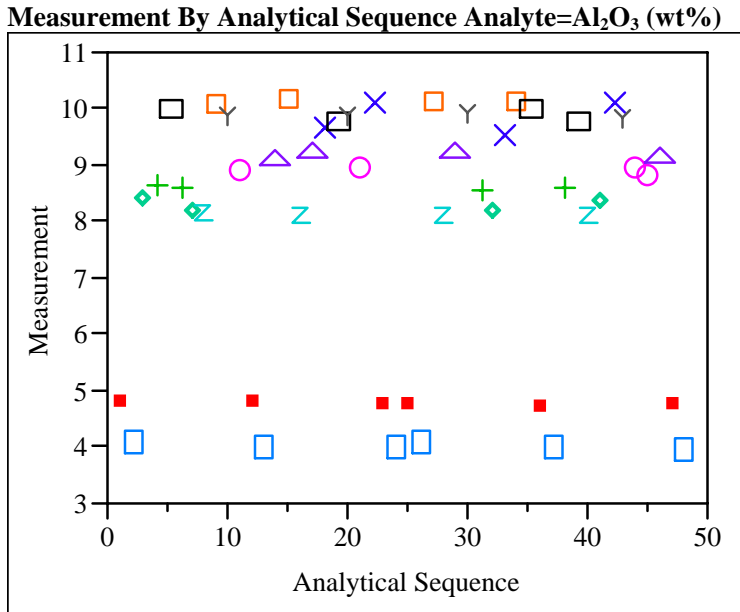

Measurement By Analytical Sequence Analyte=BaO (wt\%)

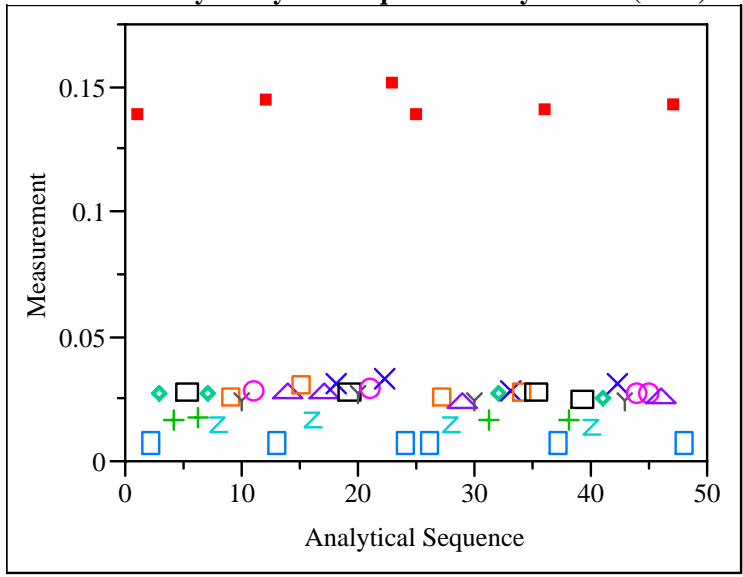

Measurement By Analytical Sequence Analyte $=\mathrm{CaO}$ (wt \%)

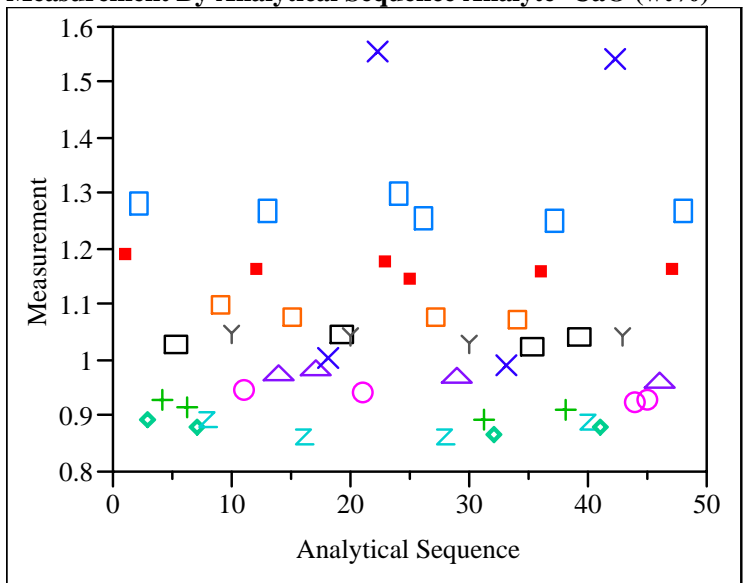

Measurement By Analytical Sequence Analyte $=\mathrm{Ce}_{2} \mathrm{O}_{3}(\mathrm{wt} \%)$

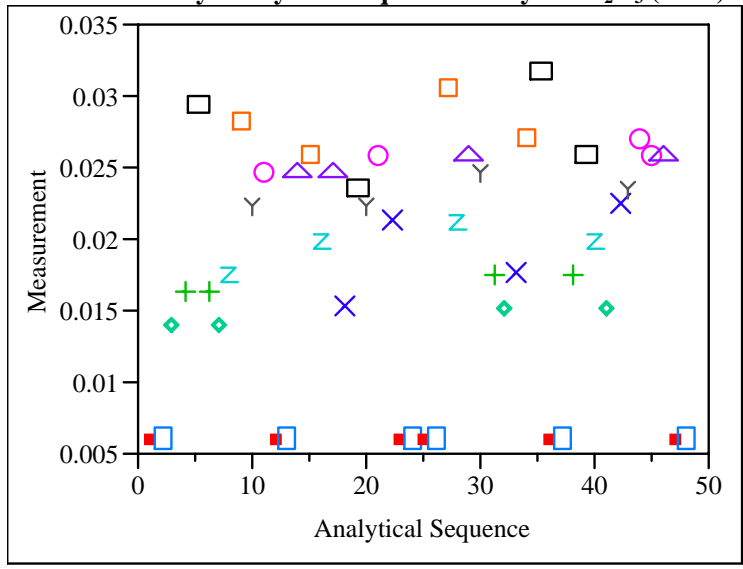

Measurement By Analytical Sequence Analyte $=\mathrm{Cr}_{2} \mathrm{O}_{3}(\mathrm{wt} \%)$

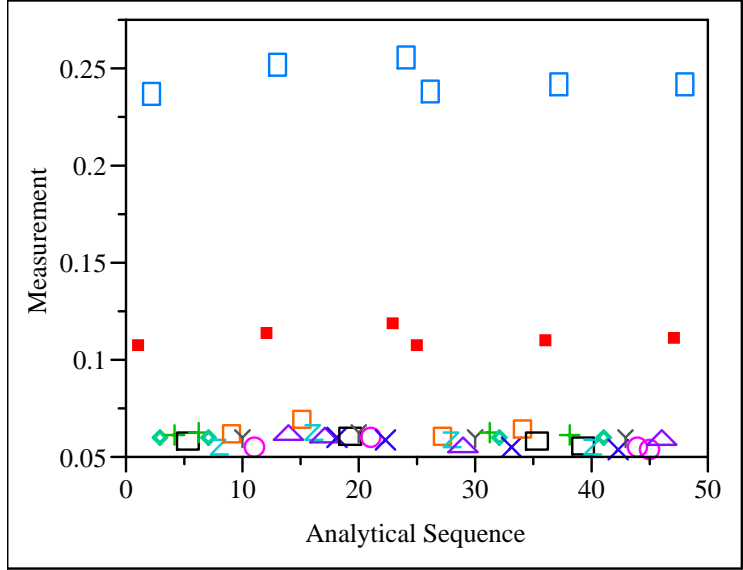

Measurement By Analytical Sequence Analyte $=\mathrm{CuO}(\mathrm{wt} \%)$

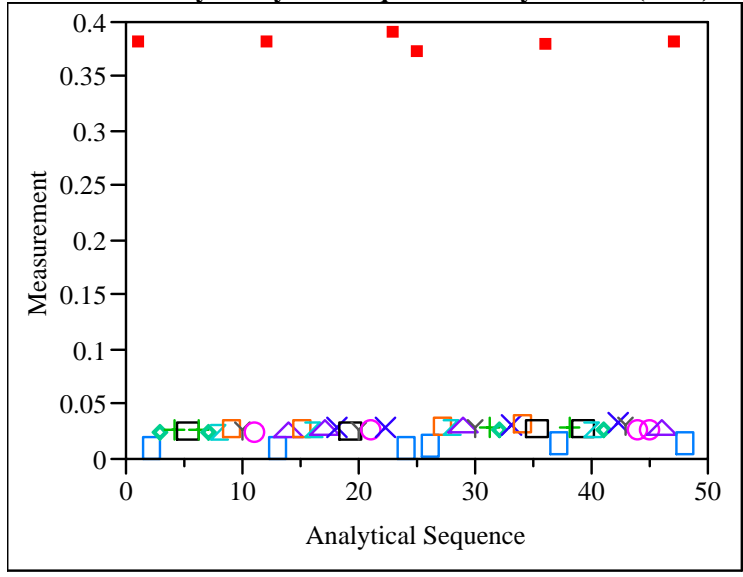




\section{Figure A1. Oxide Measurements in Analytical Sequence for Samples Prepared Using the LM Method}
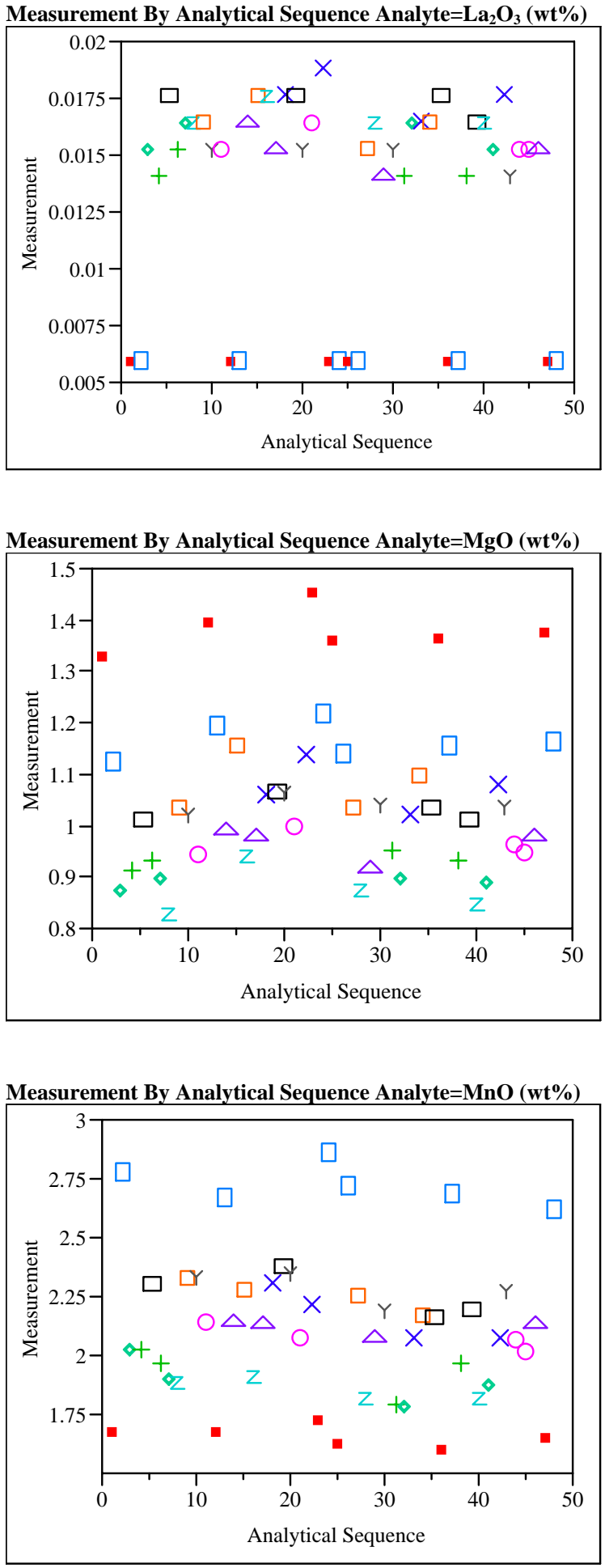

Measurement By Analytical Sequence Analyte $=\mathrm{Na}_{2} \mathrm{O}$ (wt\%)

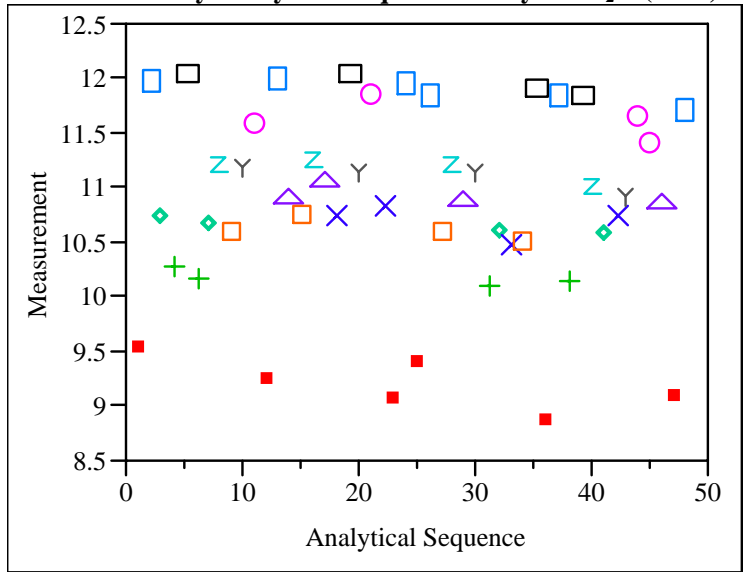

Measurement By Analytical Sequence Analyte= $\mathrm{NiO}$ (wt\%)
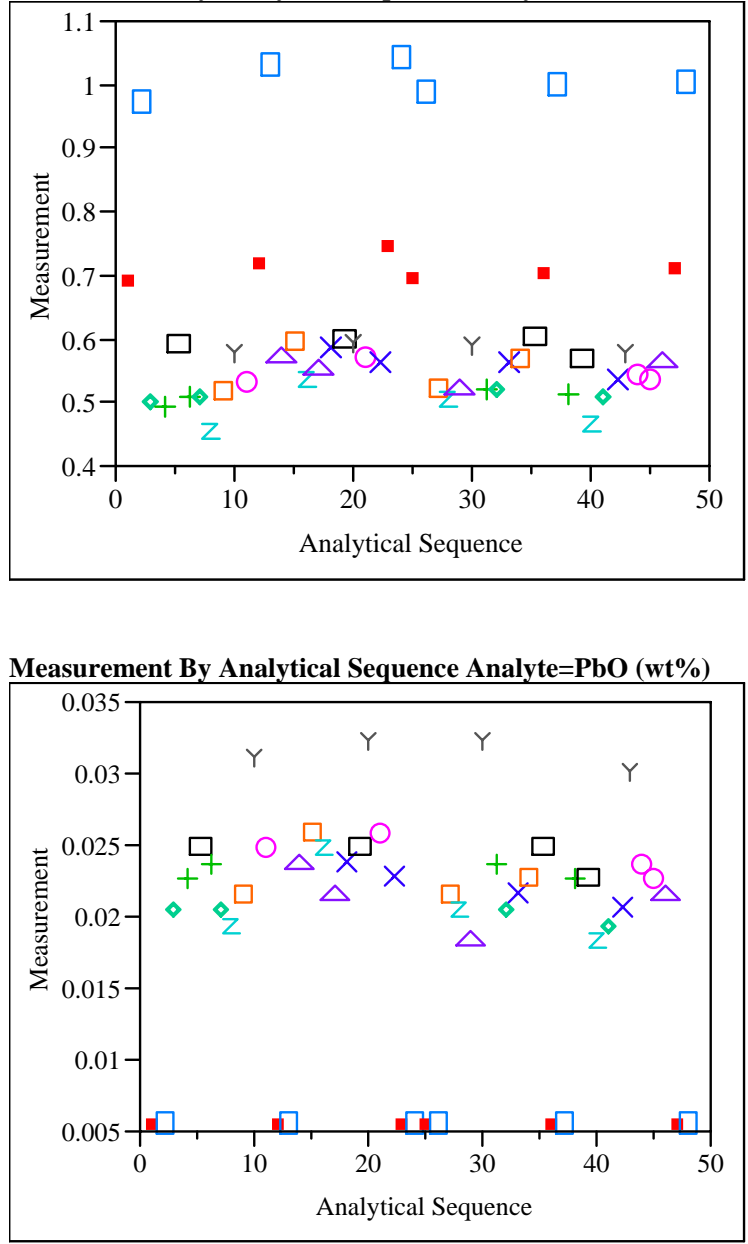


\section{Figure A1. Oxide Measurements in Analytical Sequence for Samples Prepared Using the LM Method}
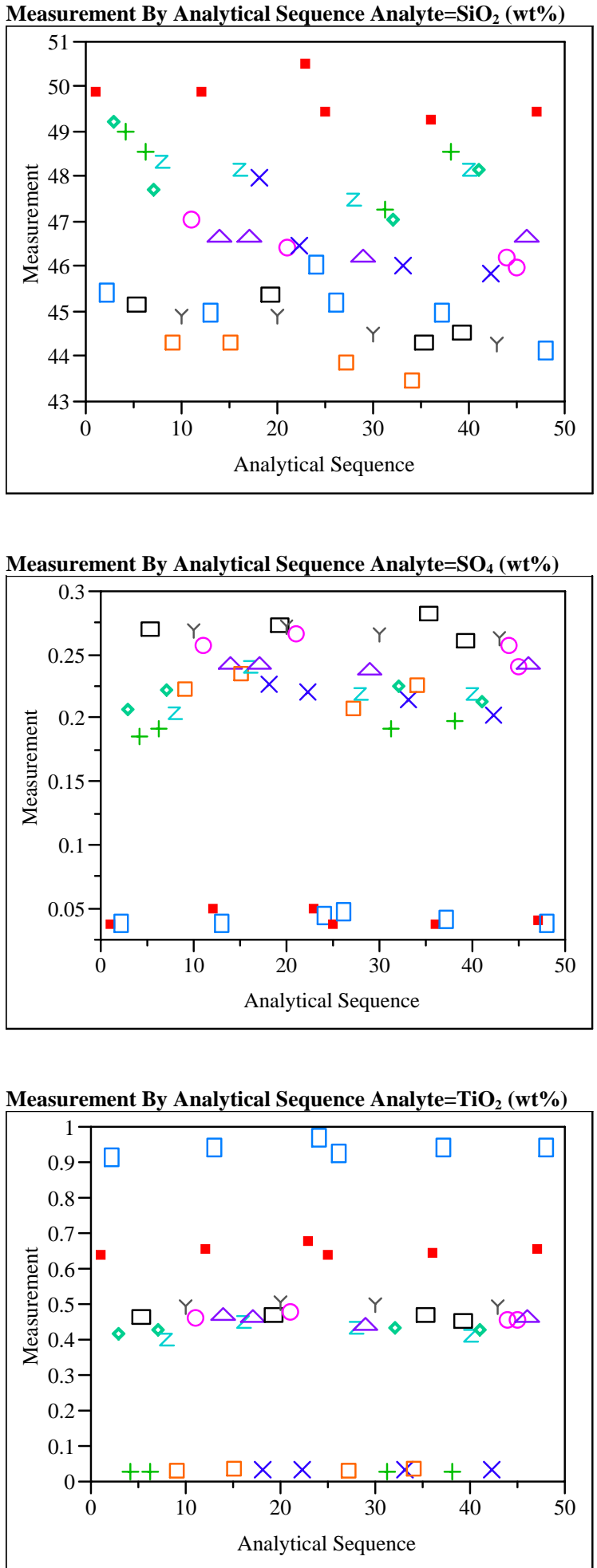

Measurement By Analytical Sequence Analyte $=\mathrm{U}_{3} \mathrm{O}_{8}(\mathrm{wt} \%)$
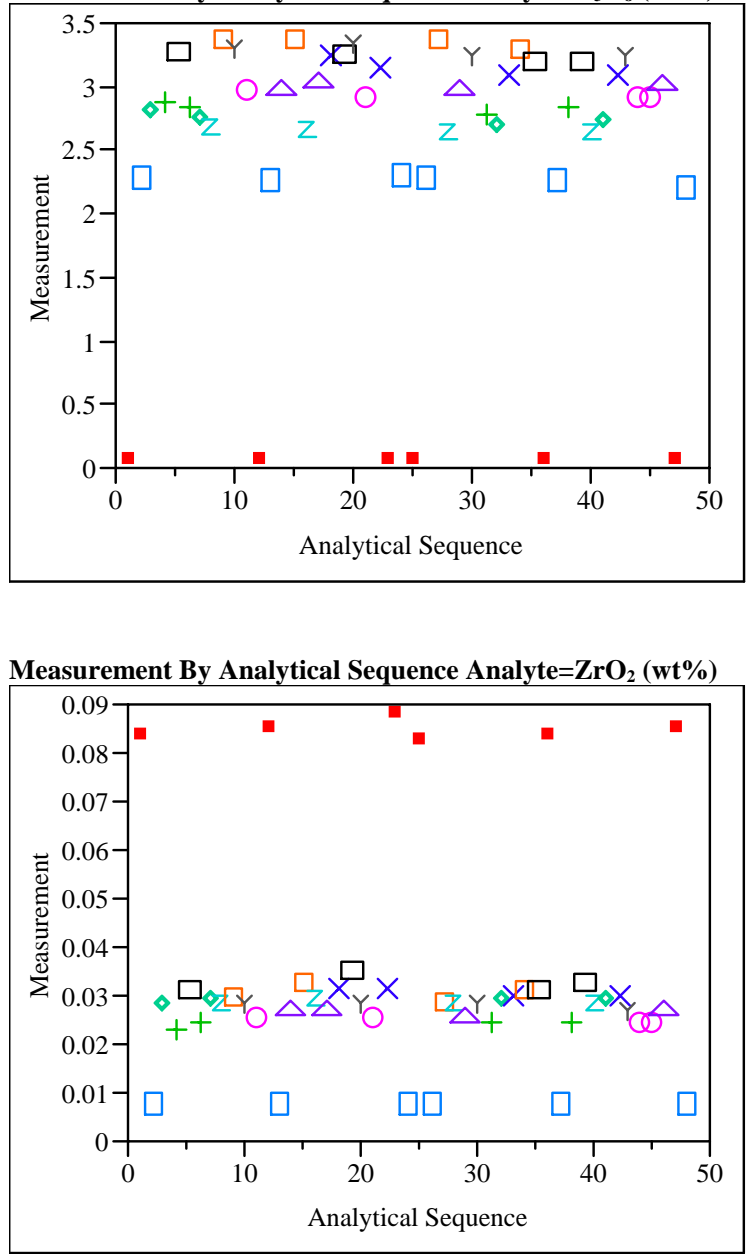


\section{Figure A2. Oxide Measurements in Analytical Sequence for Samples Prepared} Using the PF Method

\section{$\mathrm{Li}_{2} \mathrm{O}$ (wt\%) By Analytical Sequence}

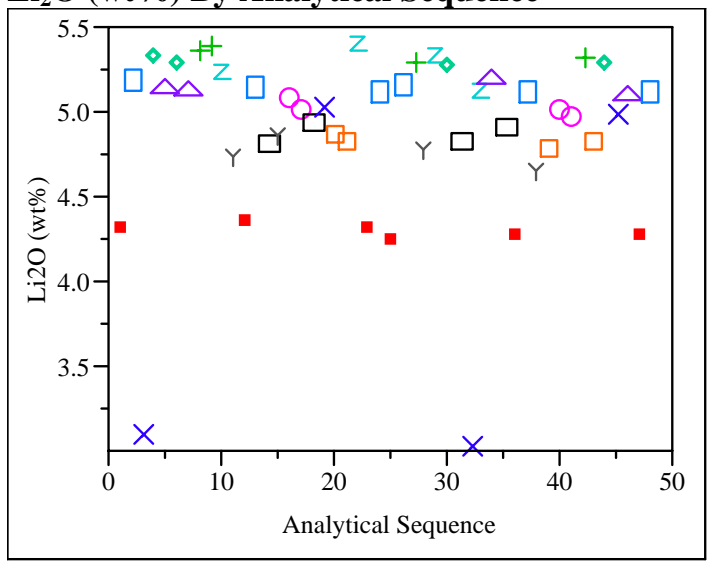

$\mathrm{Fe}_{2} \mathrm{O}_{3}$ (wt\%) By Analytical Sequence

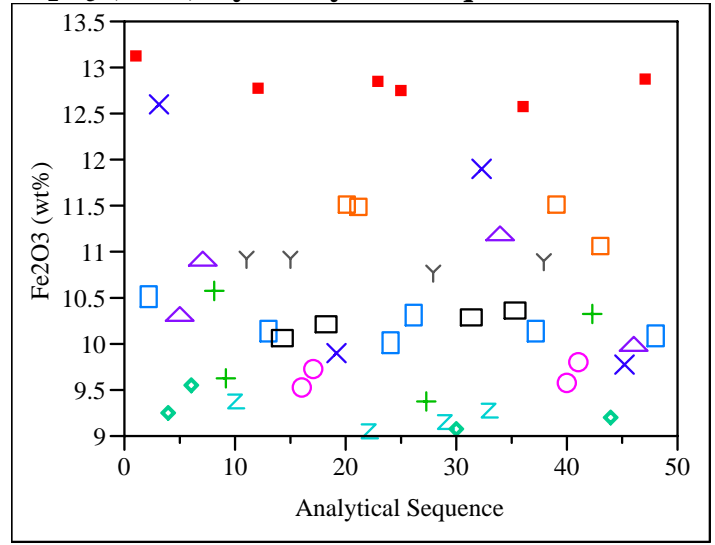

\section{$\mathrm{B}_{\mathbf{2}} \mathbf{O}_{\mathbf{3}}$ (wt\%) By Analytical Sequence}

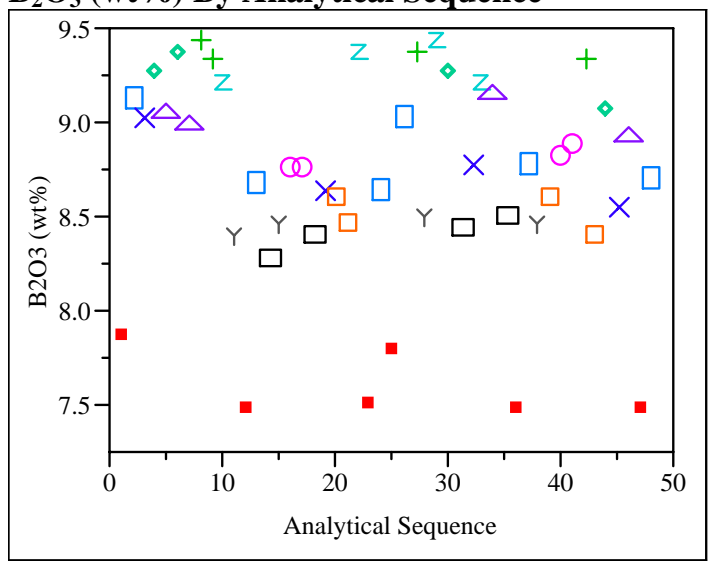


Figure A3. Oxide Measurements by Lab ID within Glass ID Including Auxiliary Measurements

for Samples Prepared Using the LM Method

\section{Variability Chart for $\mathrm{Al}_{2} \mathrm{O}_{3}$ (wt \%)}

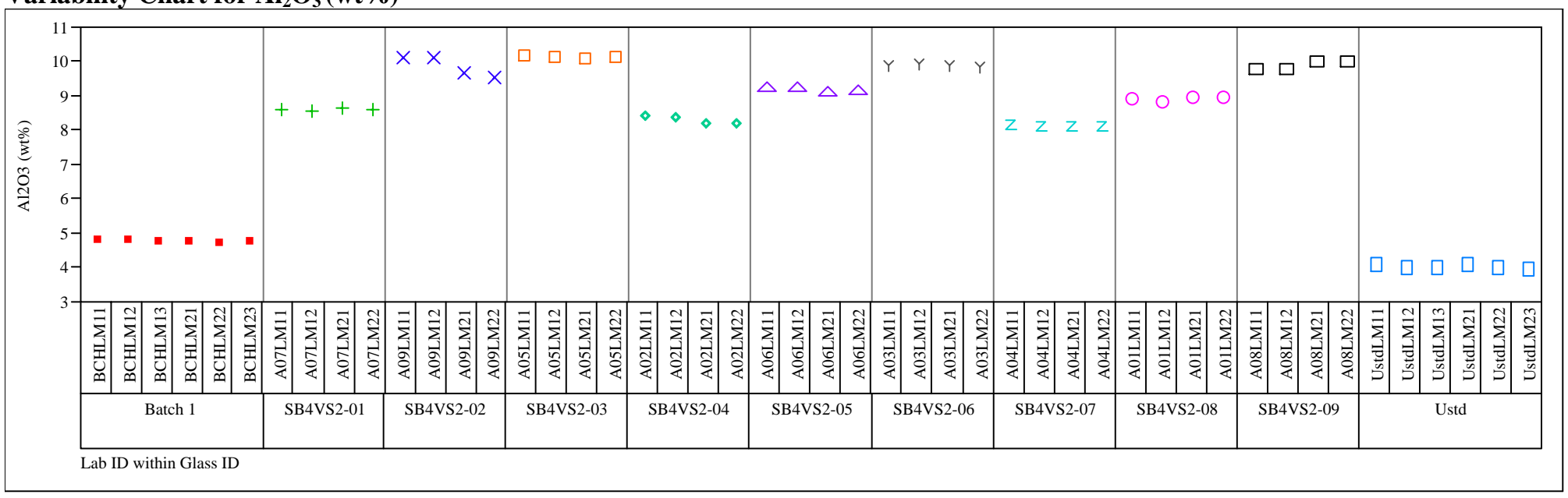

\section{Variability Chart for BaO (wt\%)}

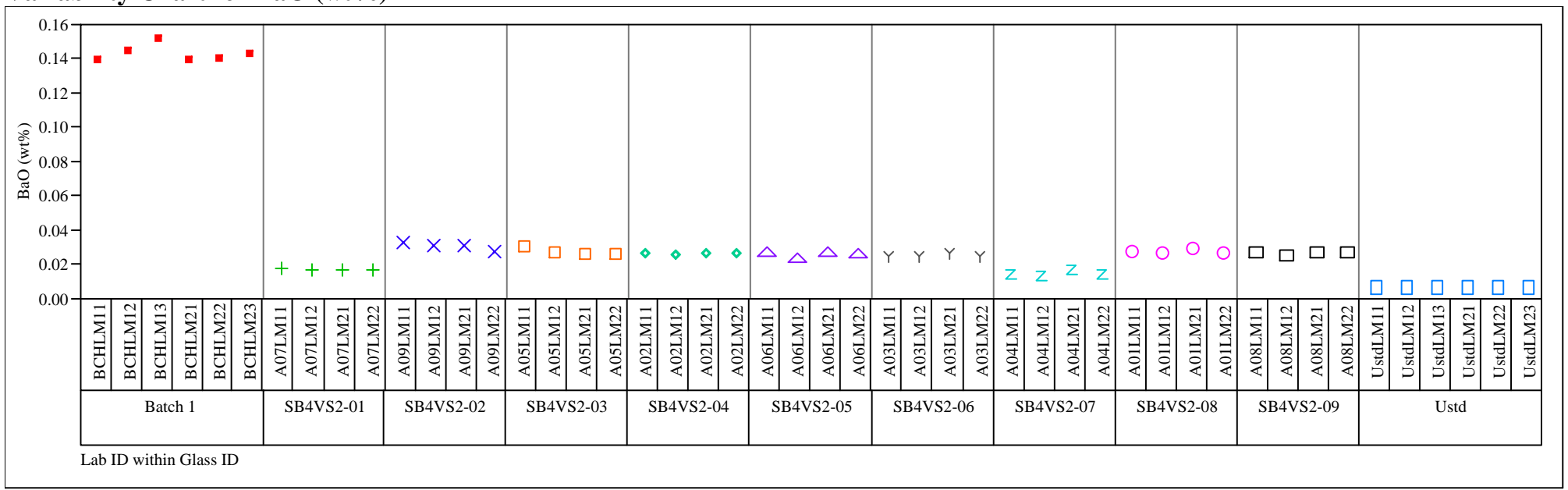


Figure A3. Oxide Measurements by Lab ID within Glass ID Including Auxiliary Measurements for Samples Prepared Using the LM Method

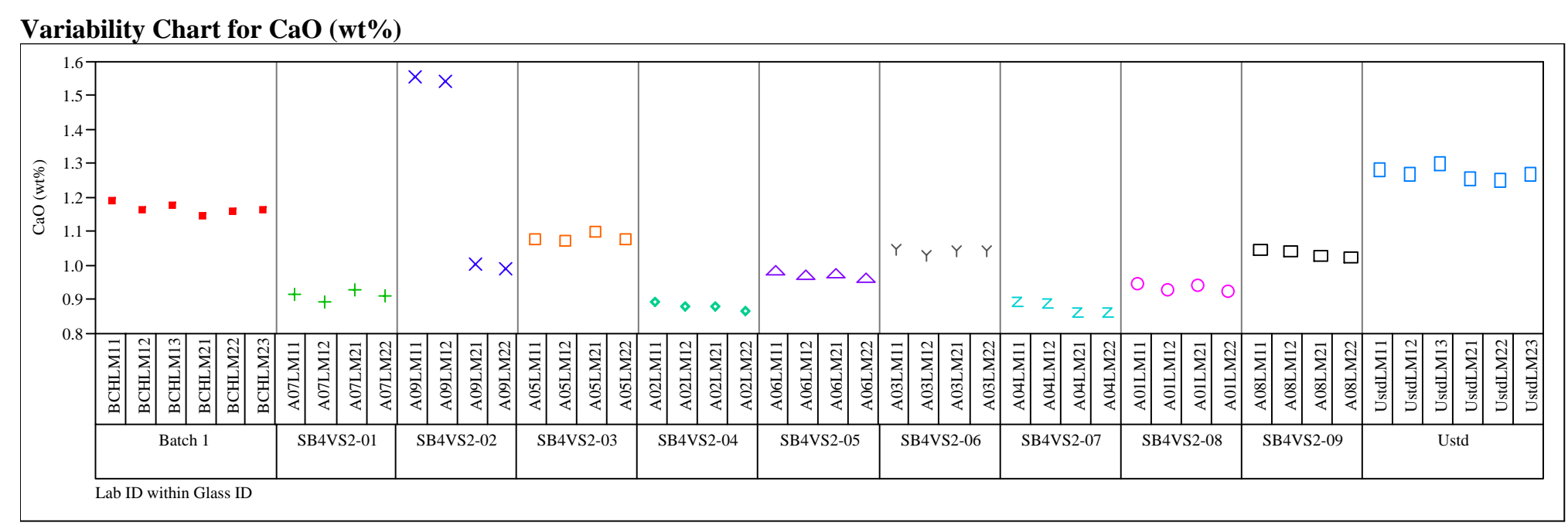

\section{Variability Chart for $\mathrm{Ce}_{2} \mathrm{O}_{3}$ (wt \%)}

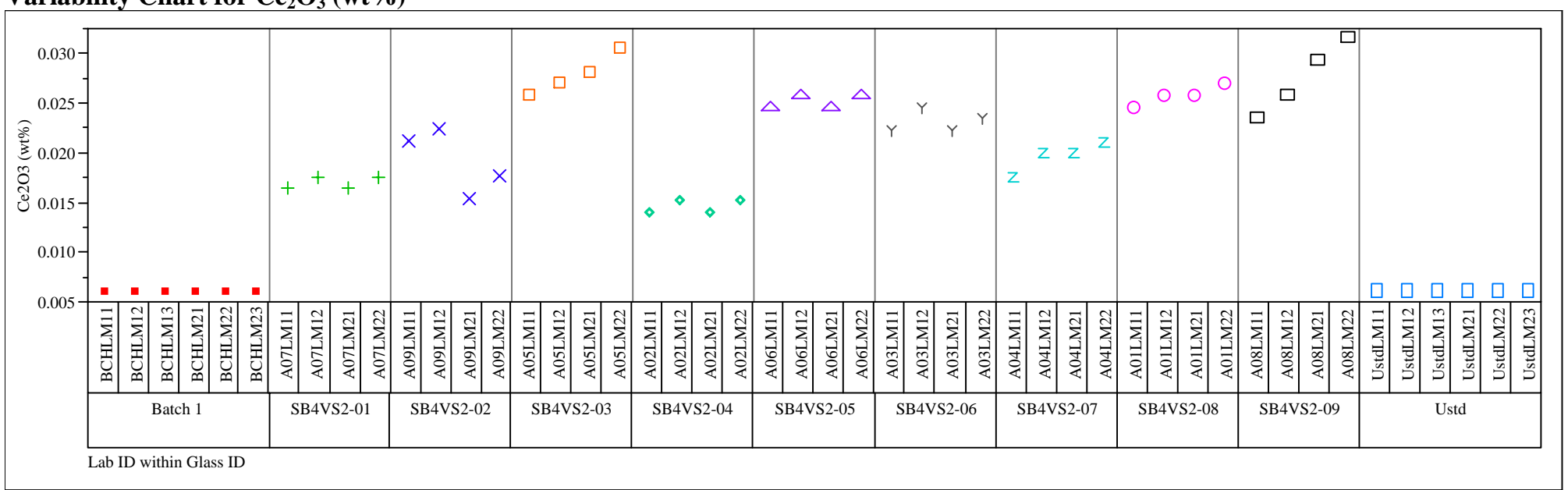


Figure A3. Oxide Measurements by Lab ID within Glass ID Including Auxiliary Measurements

for Samples Prepared Using the LM Method

\section{Variability Chart for $\mathrm{Cr}_{2} \mathrm{O}_{3}$ (wt \%)}

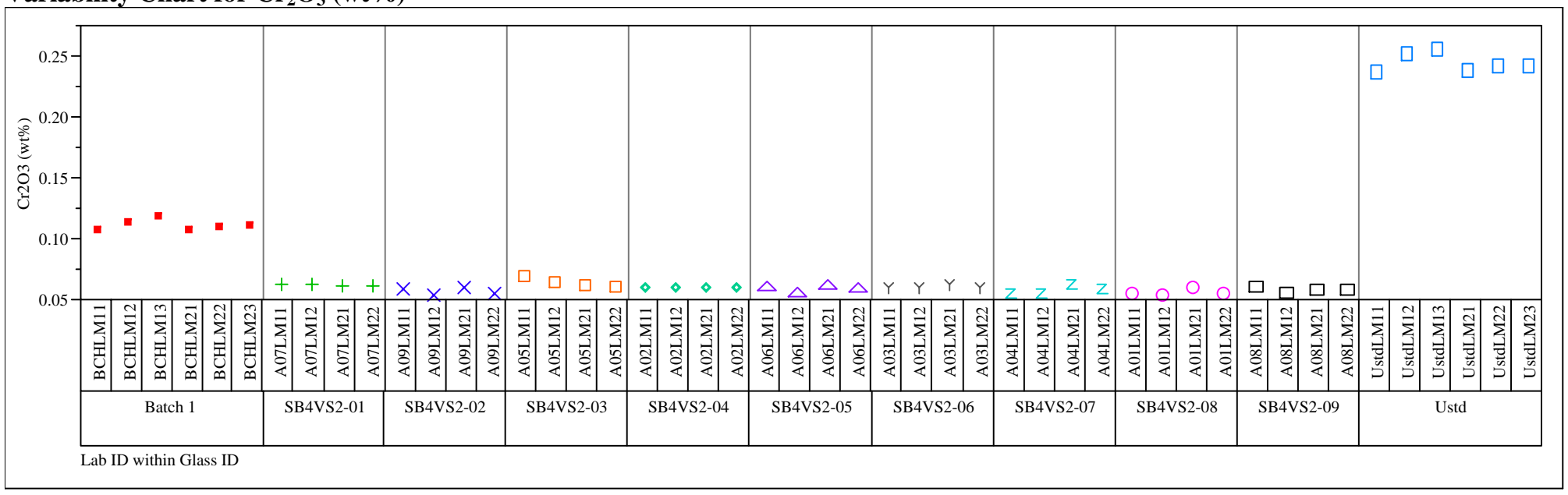

\section{Variability Chart for $\mathrm{CuO}$ (wt\%)}

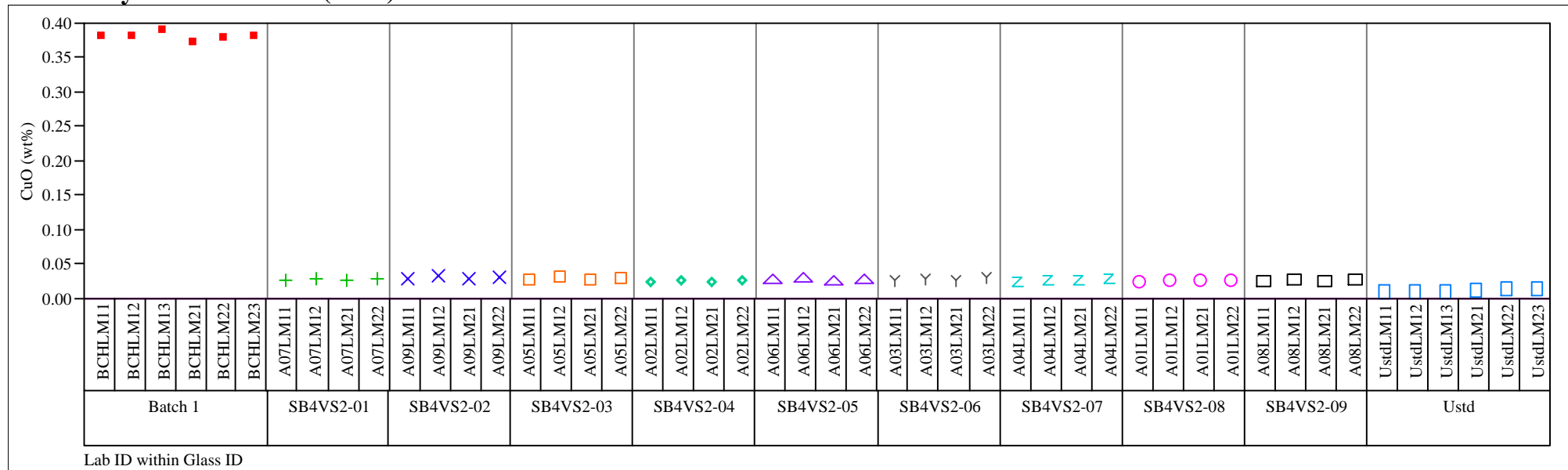


Figure A3. Oxide Measurements by Lab ID within Glass ID Including Auxiliary Measurements for Samples Prepared Using the LM Method

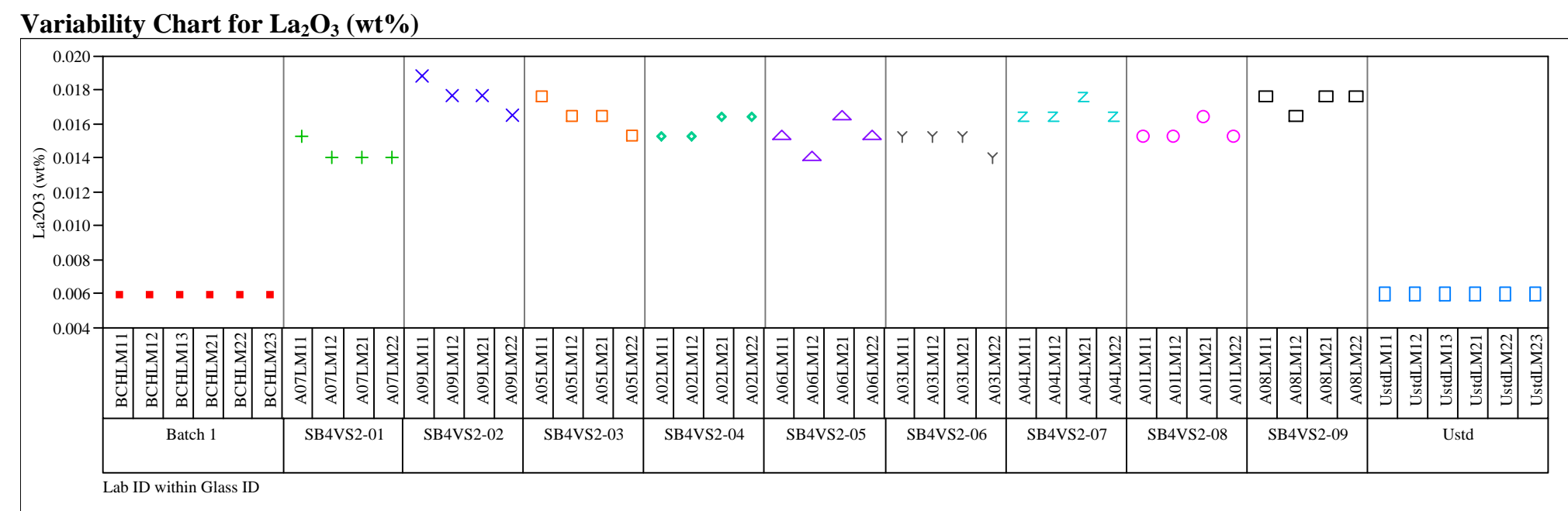

\section{Variability Chart for MgO (wt\%)}

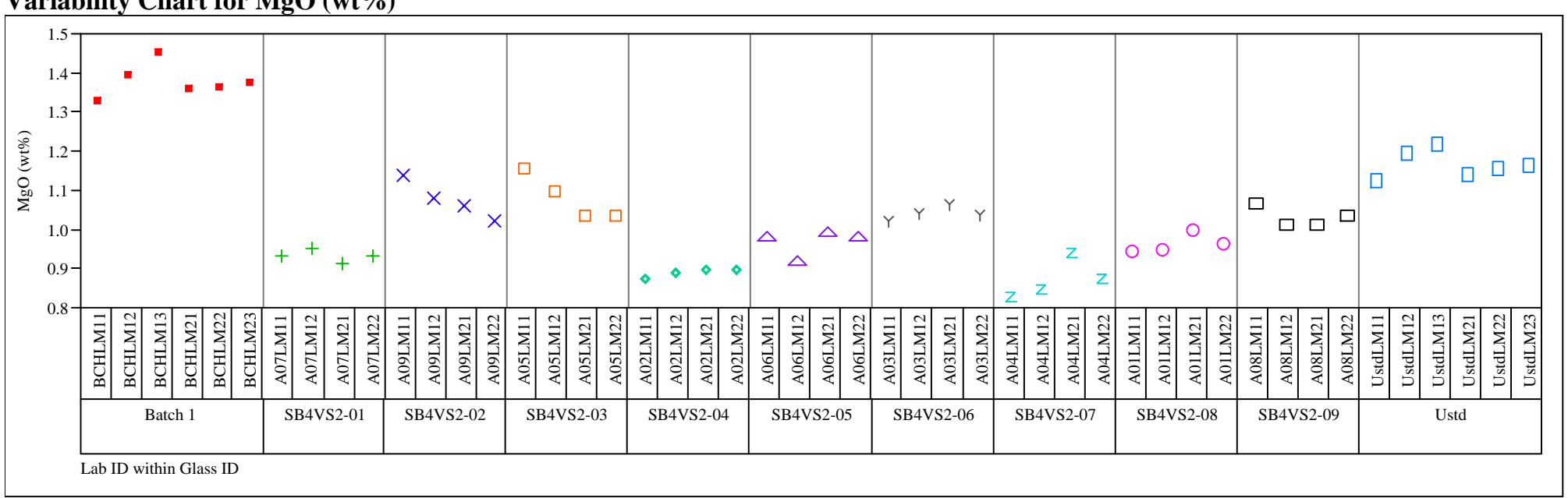


Figure A3. Oxide Measurements by Lab ID within Glass ID Including Auxiliary Measurements for Samples Prepared Using the LM Method

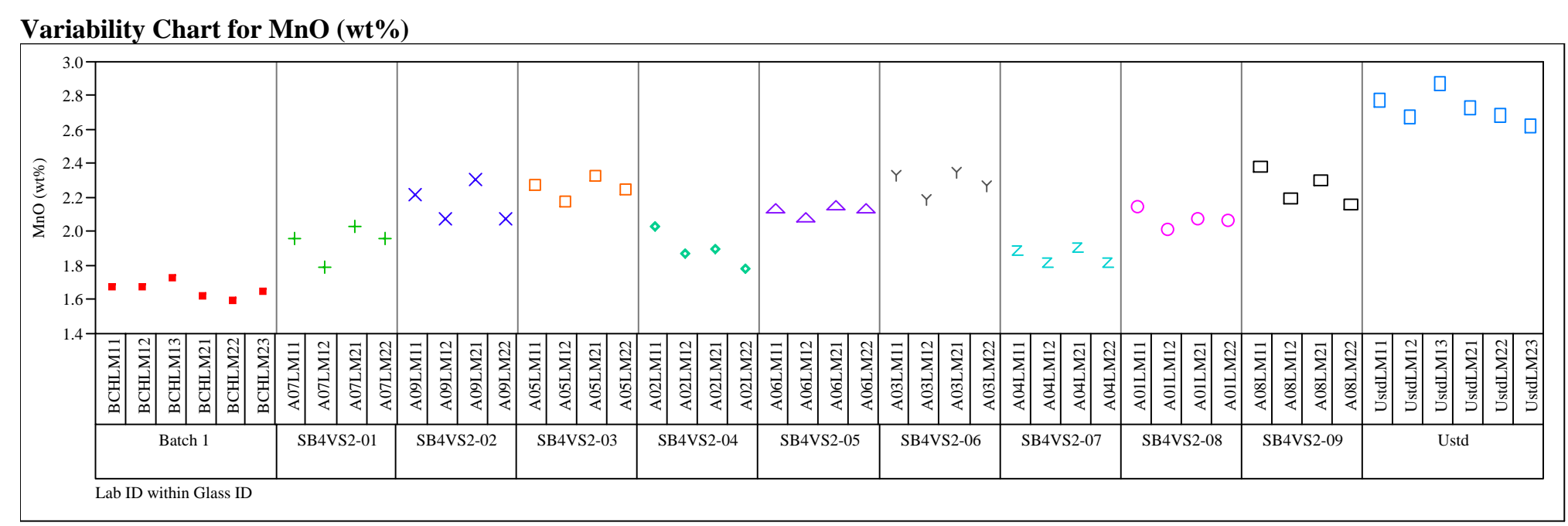

\section{Variability Chart for $\mathrm{Na}_{2} \mathrm{O}$ (wt\%)}

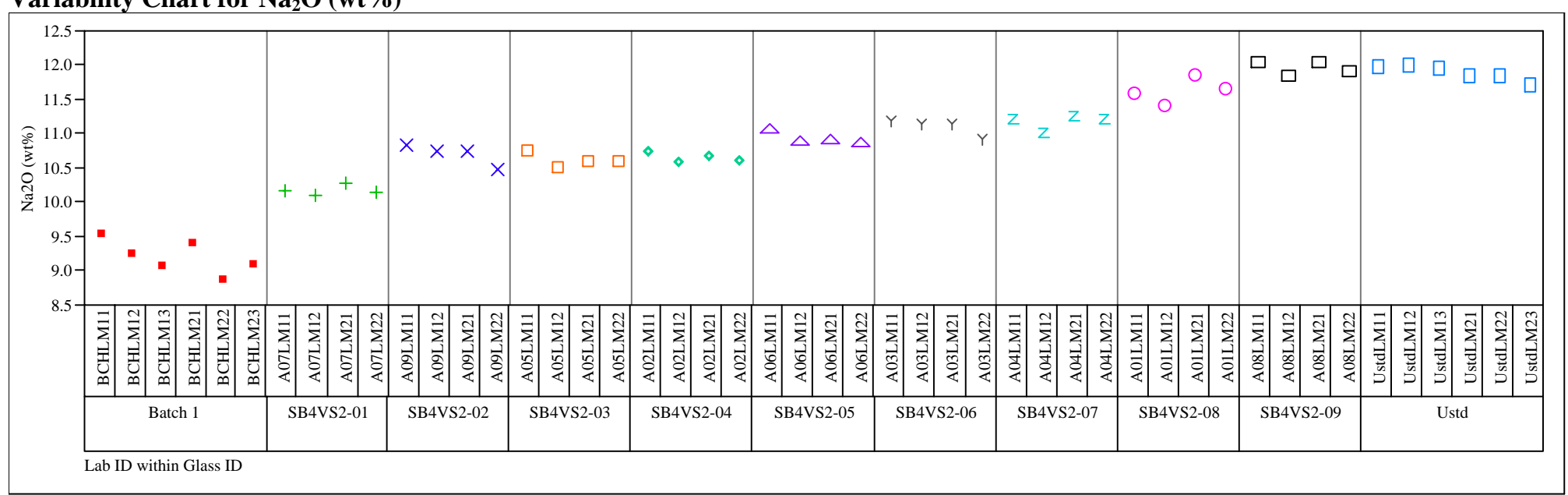


Figure A3. Oxide Measurements by Lab ID within Glass ID Including Auxiliary Measurements for Samples Prepared Using the LM Method

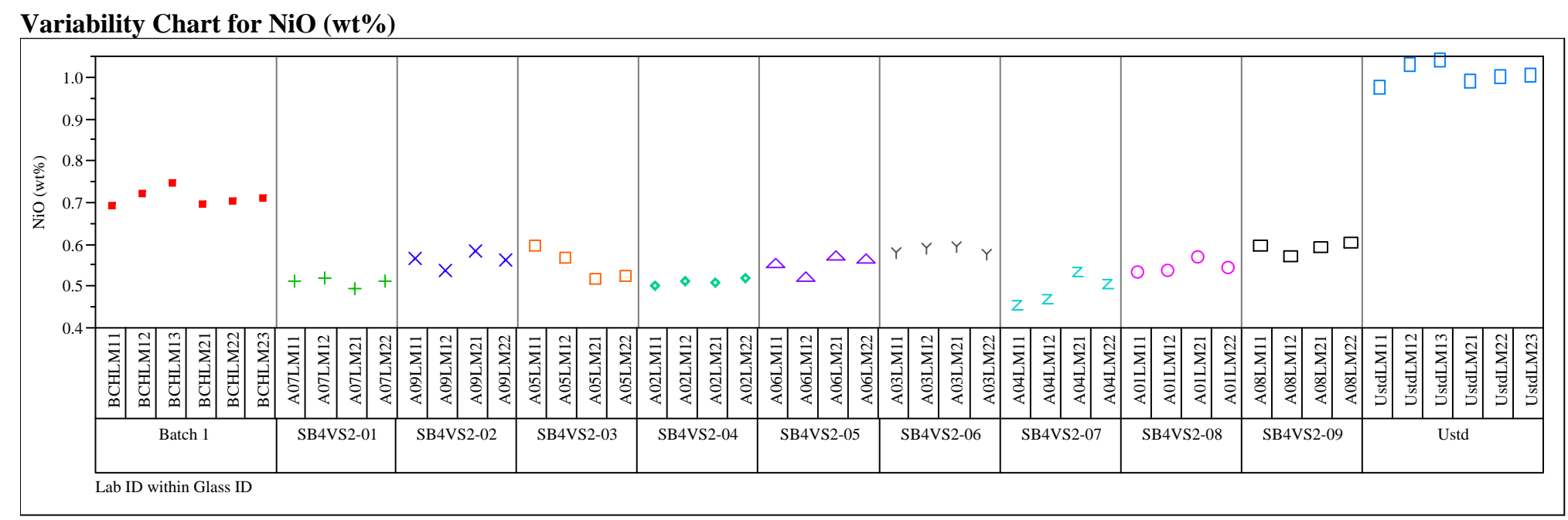

\section{Variability Chart for PbO (wt\%)}

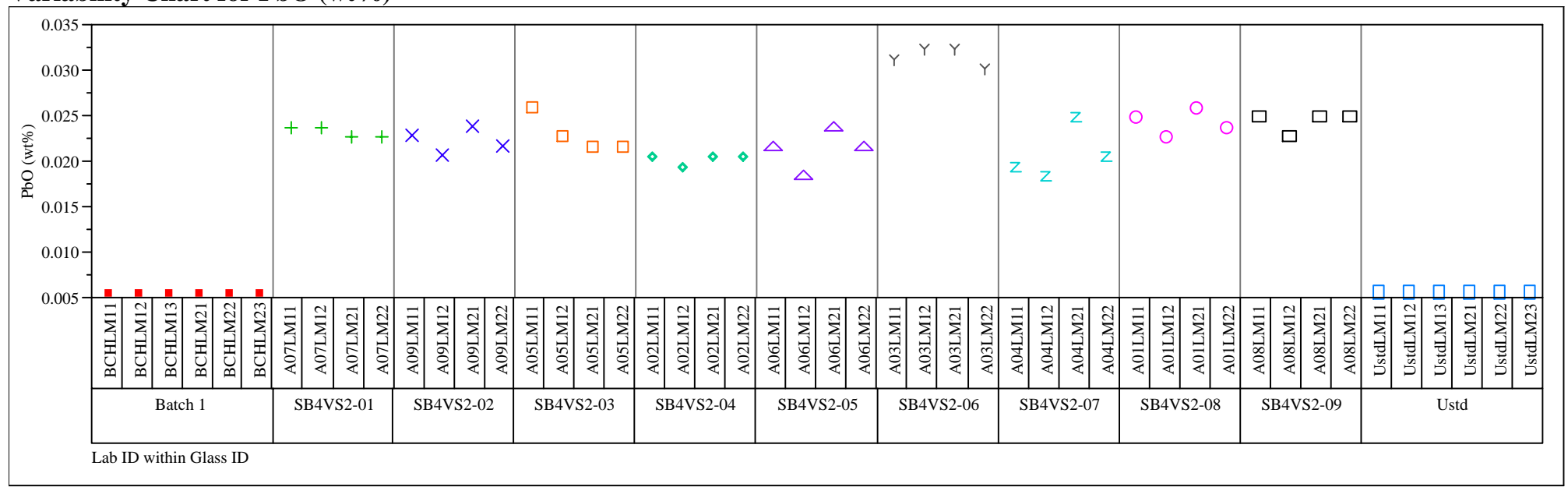


Figure A3. Oxide Measurements by Lab ID within Glass ID Including Auxiliary Measurements for Samples Prepared Using the LM Method

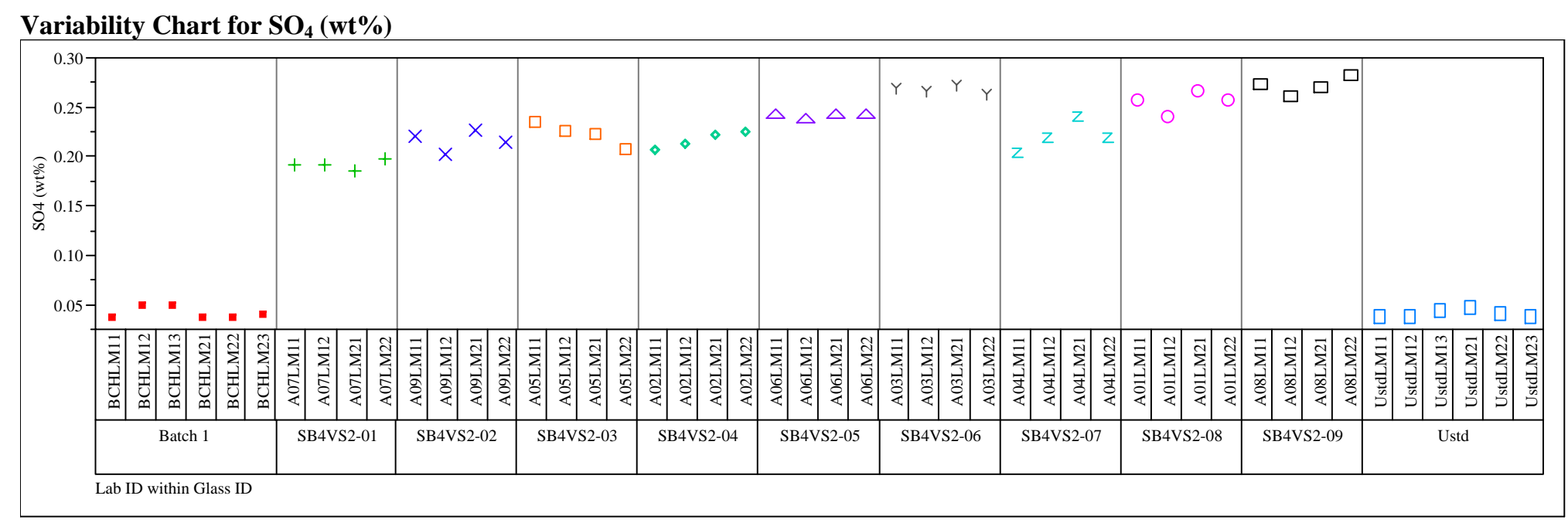

\section{Variability Chart for $\mathrm{SiO}_{2}$ (wt\%)}

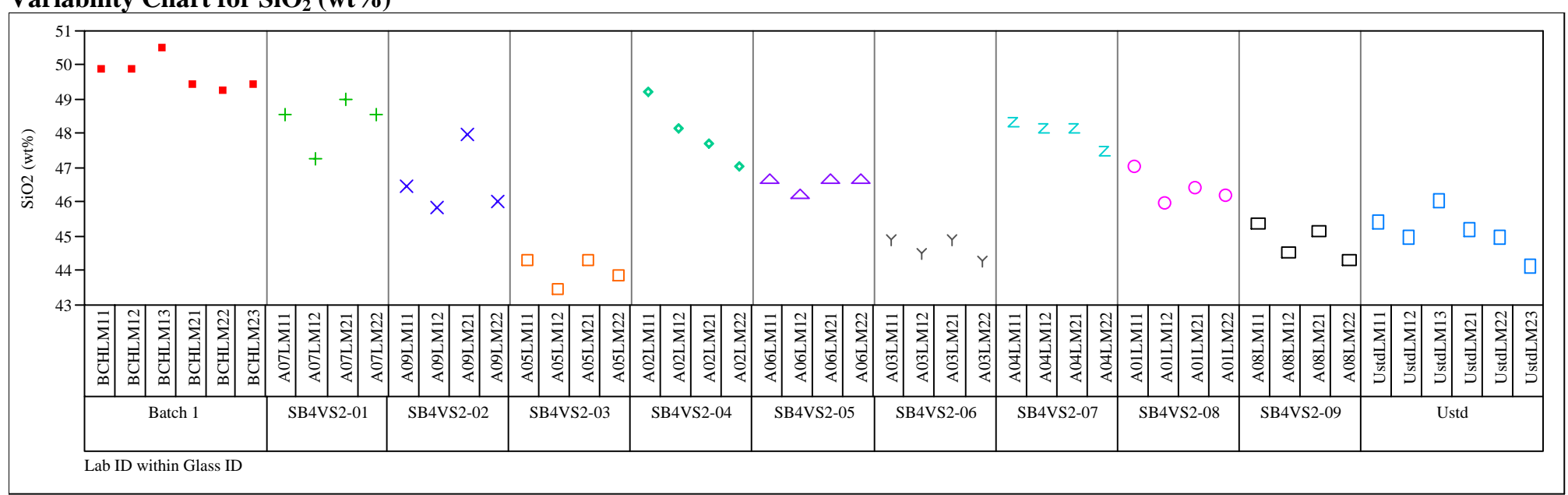


Figure A3. Oxide Measurements by Lab ID within Glass ID Including Auxiliary Measurements for Samples Prepared Using the LM Method

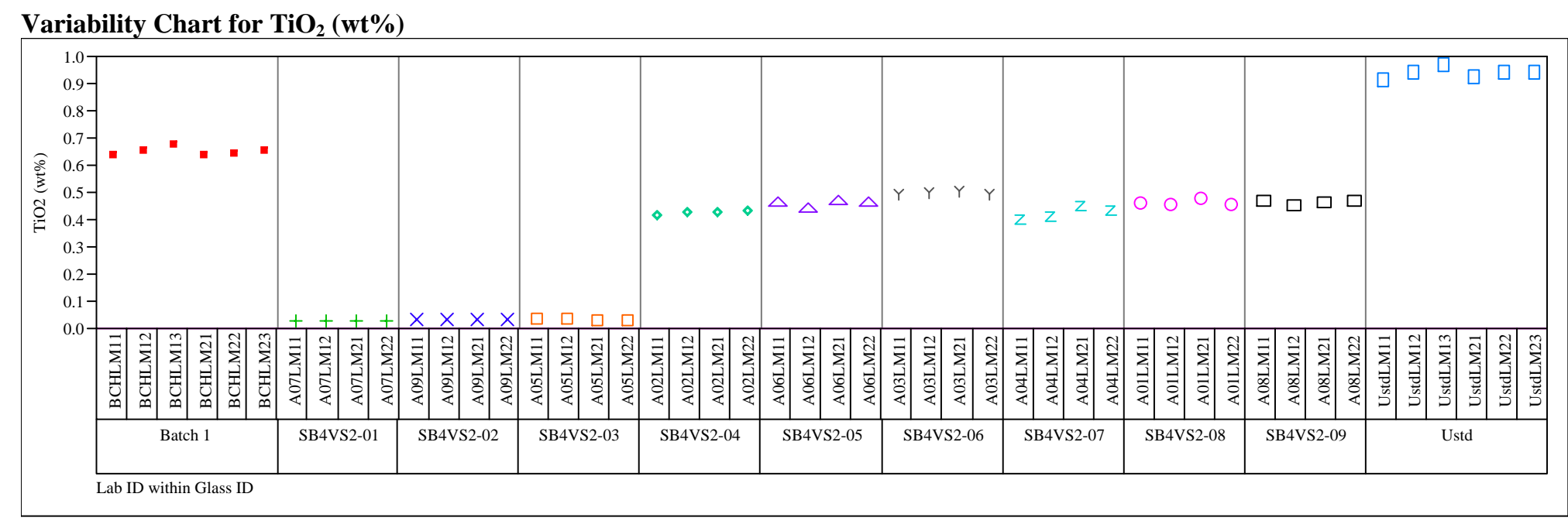

\section{Variability Chart for $\mathrm{U}_{3} \mathrm{O}_{8}$ (wt\%)}

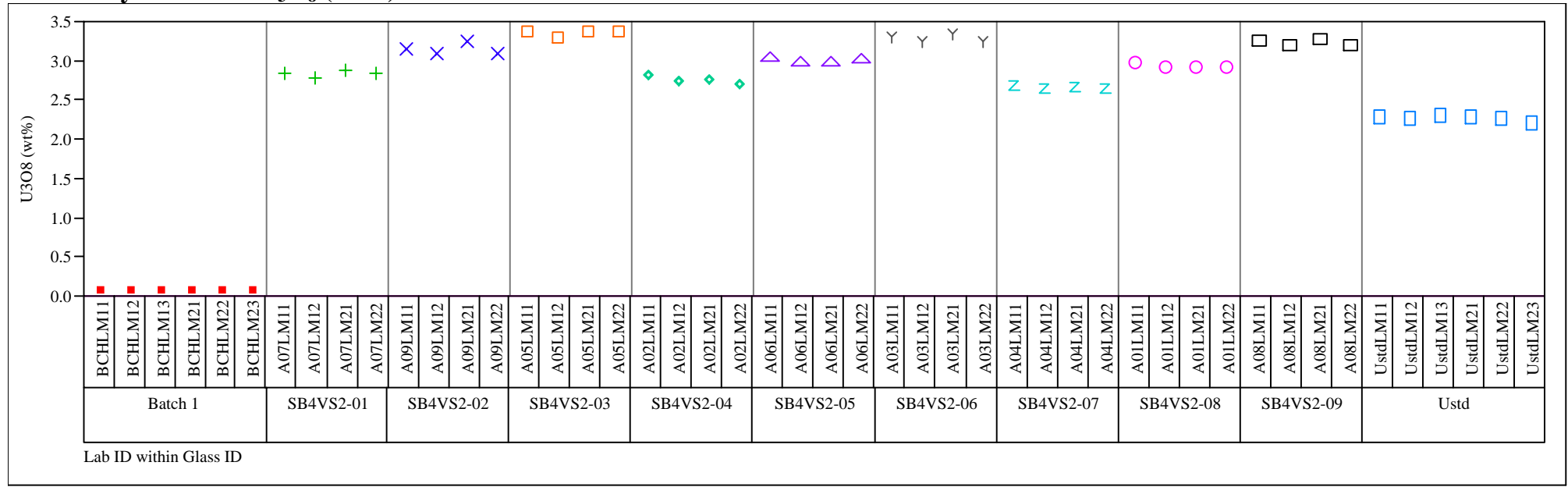




\section{Figure A3. Oxide Measurements by Lab ID within Glass ID Including Auxiliary Measurements} for Samples Prepared Using the LM Method

Variability Chart for $\mathrm{ZrO}_{2}$ (wt \%)

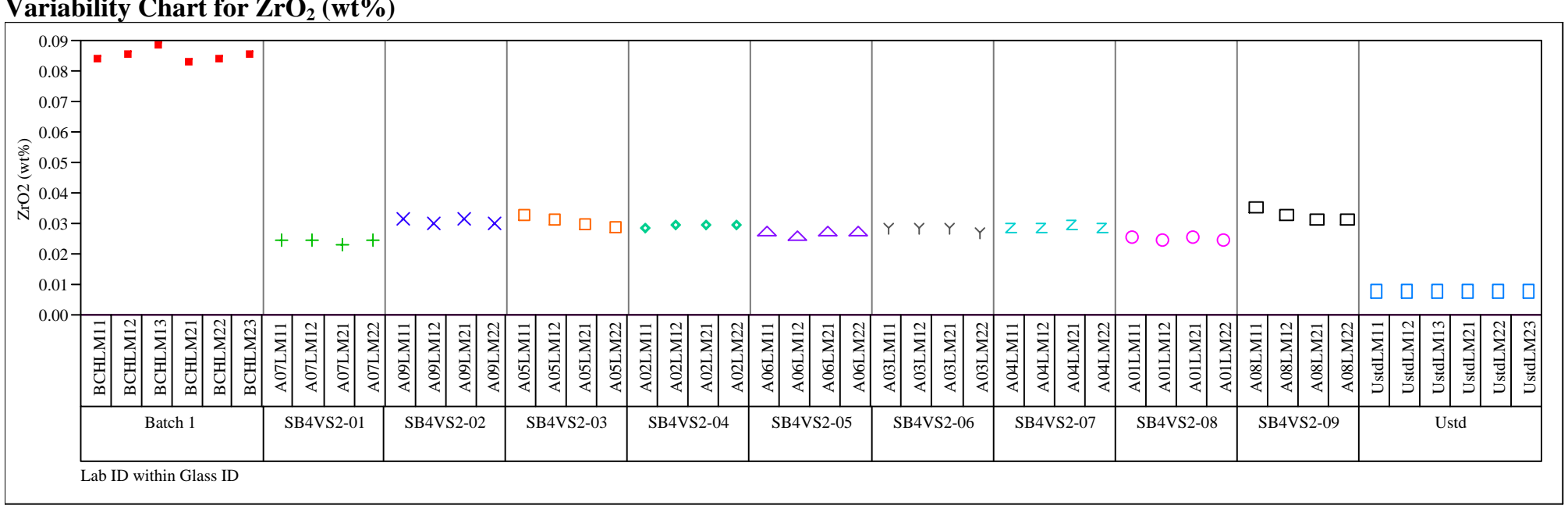


Figure A4. Oxide Measurements by Lab ID within Glass ID Including Auxiliary Measurements for Samples Prepared Using the PF Method

Variability Chart for $\mathrm{B}_{2} \mathrm{O}_{3}$ (wt\%)

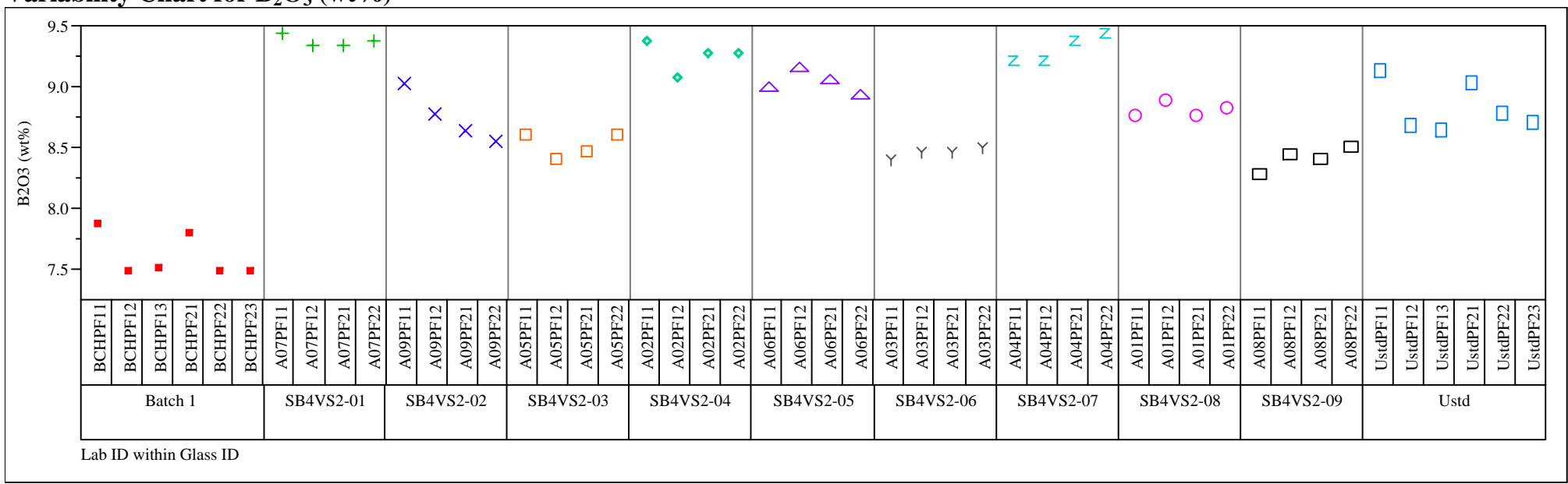

\section{Variability Chart for $\mathrm{Fe}_{2} \mathrm{O}_{3}$ (wt\%)}

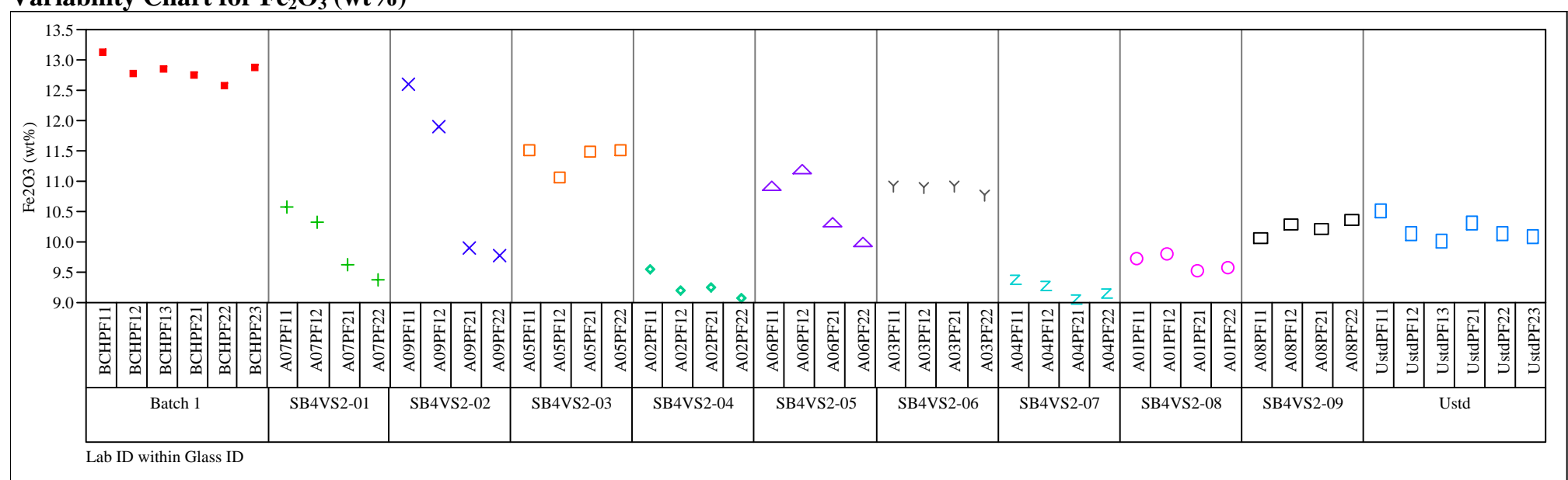


Figure A4. Oxide Measurements by Lab ID within Glass ID Including Auxiliary Measurements for Samples Prepared Using the PF Method

Variability Chart for $\mathrm{Li}_{2} \mathrm{O}$ (wt \%)

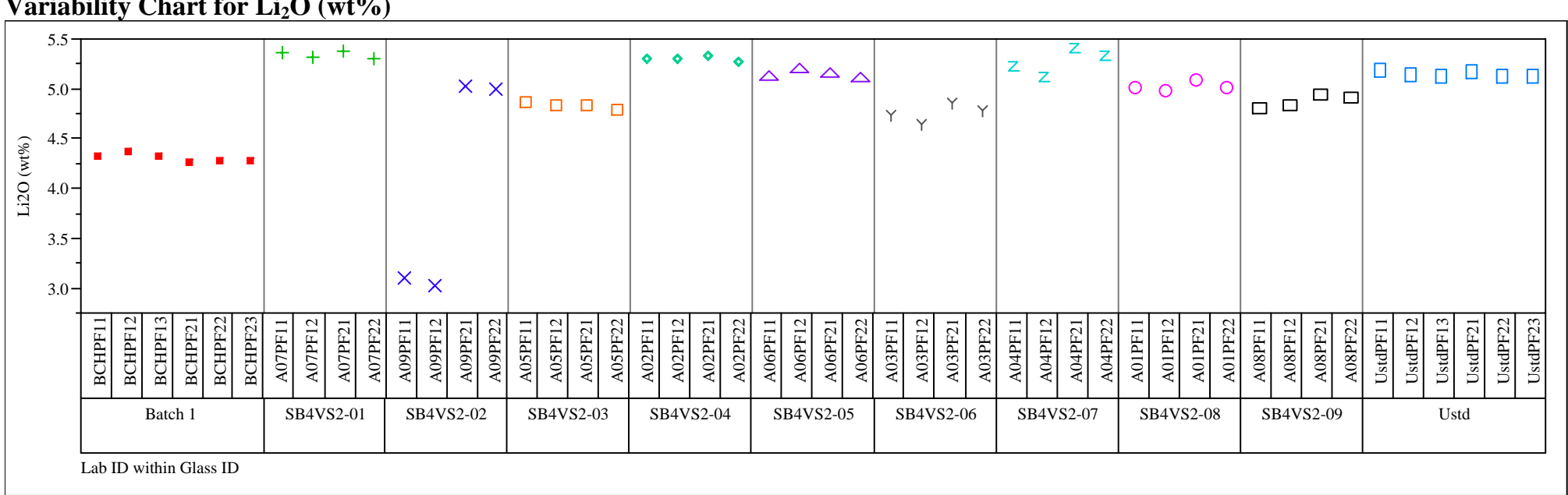




\section{Figure A5. PSAL Measurements by Analytical Block for Samples of the Standard Glasses Prepared Using the LM Method}

Oneway Analysis of Measurement By Block Glass ID=Batch 1, Oxide $=\mathrm{Al}_{2} \mathrm{O}_{3}(\mathrm{wt} \%)$

Reference Value $=4.877 \mathrm{wt} \%$

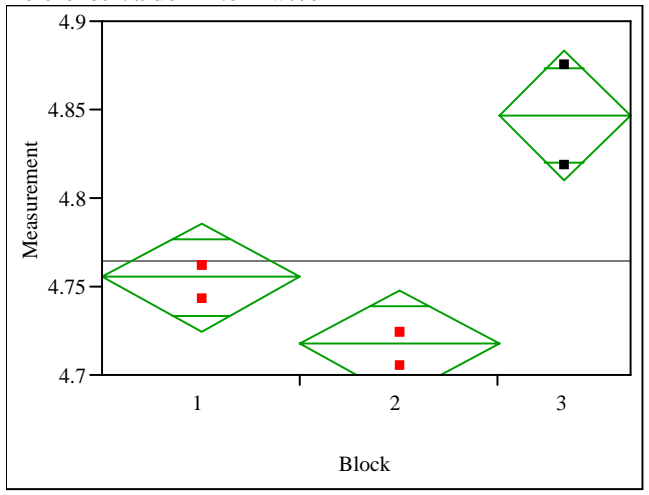

Oneway Anova

Summary of Fit

Adj Rsquare

0.907223

0.870113

0.020409

Mean of Response 4.763902
8

Analysis of Variance

Source DF Sum of Squares Mean Square F Ratio Prob $>$ F

$\begin{array}{lllllll}\text { Block } & 2 & 0.02036507 & 0.010183 & 24.4464 & 0.0026\end{array}$

$\begin{array}{llll}\text { Error } & 5 & 0.00208262 & 0.000417\end{array}$

C. Total $7 \quad 0.02244770$

Means for Oneway Anova

Level Number Mean Std Error Lower 95\% Upper 95\%

$\begin{array}{lllllr}1 & 3 & 4.75524 & 0.01178 & 4.7250 & 4.7855\end{array}$

$\begin{array}{llllll}2 & 3 & 4.71745 & 0.01178 & 4.6872 & 4.7477 \\ 3 & 2 & 4.84657 & 0.01443 & 4.8095 & 4.8837\end{array}$

Std Error uses a pooled estimate of error variance
Oneway Analysis of Measurement By Block Glass ID=Batch 1, Oxide $=\mathrm{BaO}(\mathrm{wt} \%)$

Reference Value $=0.151 \mathrm{wt} \%$

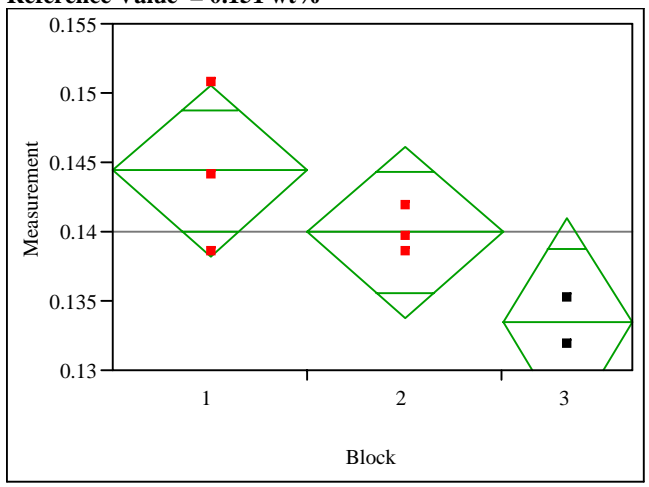

Oneway Anova

Summary of Fit

Rsquare

Adj Rsquare

Root Mean Square

Mean of Response

Observations (or Sum Wgts)

0.624299

0.474019

0.139981

Analysis of Variance

Source DF Sum of Squares Mean Square F Ratio Prob > F

$\begin{array}{llllll}\text { Block } & 2 & 0.00014465 & 0.000072 & 4.1542 & 0.0865\end{array}$

Error $5-0.00008705$

$\begin{array}{lll}0.000072 & 4.1542 & 0.0865 \\ 0.000017 & \end{array}$

C. Total 7

Means for Oneway Anova

Level Number Mean Std Error Lower 95\% Upper 95\%

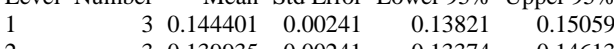

$\begin{array}{llllll}1 & 3 & 0.139935 & 0.00241 & 0.13374 & 0.14613 \\ 3 & 2 & 0.13422 & 0.0025 & 0.12584 & 0.14101\end{array}$

Std Error uses a pooled estimate of error variance
Oneway Analysis of Measurement By Block Glass ID=Batch 1, Oxide $=\mathrm{CaO}(\mathrm{wt} \%)$

Reference Value $=1.220$ wt $\%$

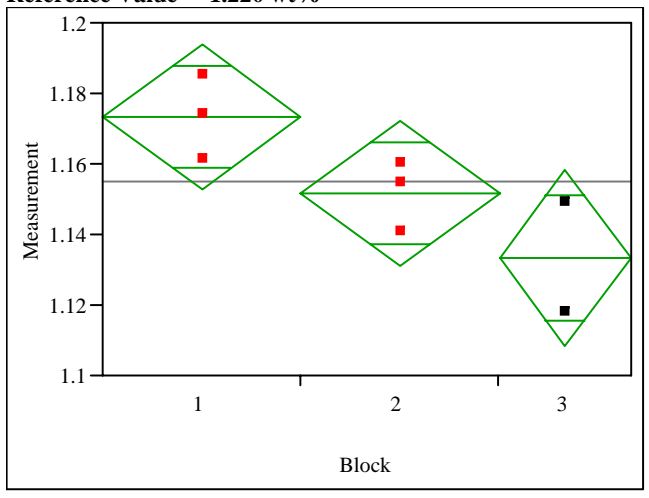

Oneway Anova

Summary of Fit

Adj Rsquare $\quad 0.54504$

Root Mean Square Error $\quad 0.013861$

Mean of Response

1.155215

Analysis of Variance

Source DF Sum of Squares Mean Square F Ratio Prob > F $\begin{array}{llllll}\text { Block } & 2 & 0.00199537 & 0.000998 & 5.1930 & 0.0602\end{array}$ $\begin{array}{llll}\text { Error } & 5 & 0.00096061 & 0.000192\end{array}$

C. Total $7 \quad 0.00295597$

Means for Oneway Anova

Level Number Mean Std Error Lower 95\% Upper 95\% $1 \quad 3 \quad 1.17346 \quad 0.00800 \quad 1.1529 \quad 1.1940$ $\begin{array}{llllll}2 & 3 & 1.15154 & 0.00800 & 1.1310 & 1.1721 \\ 3 & 2 & 1.13335 & 0.00980 & 1.1082 & 1.1585\end{array}$

Std Error uses a pooled estimate of error variance 


\section{Figure A5. PSAL Measurements by Analytical Block for Samples of the Standard Glasses Prepared Using the LM Method}

Oneway Analysis of Measurement By Block Glass ID=Batch 1, $\mathrm{Oxide}=\mathrm{Ce}_{2} \mathrm{O}_{3}(\mathrm{wt} \%)$

Reference Value $=0.0 \mathrm{wt} \%$

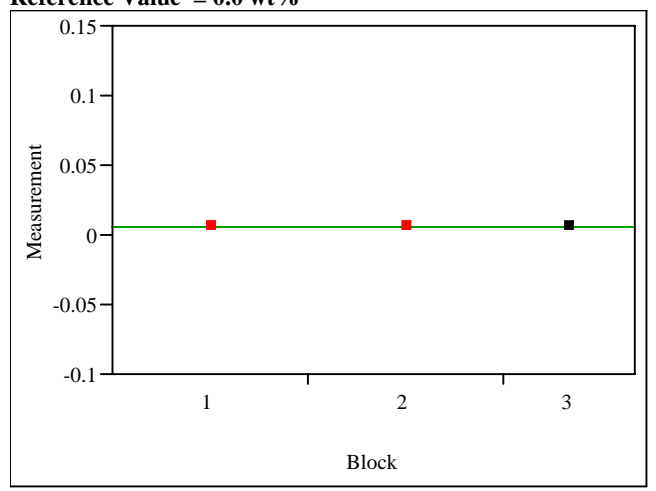

Oneway Anova

Summary of Fit

Rsquare

Adj Rsquare

Root Mean Square Emor

$\begin{array}{lr}\text { Mean of Response } & 0.005857 \\ \text { Observations (or Sum Wgts) } & 8\end{array}$

Analysis of Variance

Source DF Sum of Squares Mean Square F Ratio Prob > F

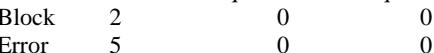

C. Total 7

\section{0}

Means for Oneway Anova

Level Number Mean Std Error Lower 95\% Upper 95\%

$\begin{array}{llrrrr}1 & 3 & 0.005857 & 0 & 0.00586 & 0.00586\end{array}$

$\begin{array}{llllll}2 & 3 & 0.005857 & 0 & 0.00586 & 0.00586\end{array}$

Std Error uses a pooled estimate of error variance
Oneway Analysis of Measurement By Block Glass ID=Batch 1, Oxide $=\mathrm{Cr}_{2} \mathrm{O}_{3}$ (wt \%)

Reference Value $=0.107$ wt $\%$

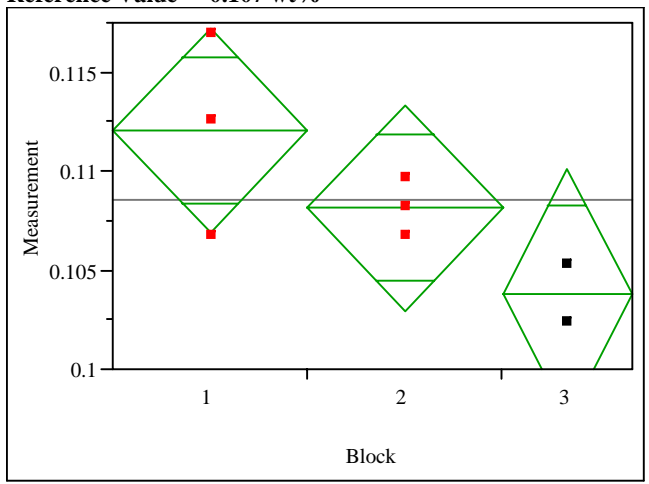

Oneway Anova

Summary of Fit

Rsquare

Adj Rsquare

Root Mean Squa

Mean of Response

Observations (or Sum Wgts) $\quad 8$

0.575309

0.405432

0.0035

Analysis of Variance

Source DF Sum of Squares Mean Square F Ratio Prob $>$ F

$\begin{array}{llllll}\text { Block } & 2 & 0.00008296 & 0.000041 & 3.3866 & 0.1175\end{array}$

Error $5-0.00006124$

$\begin{array}{lll}0.000041 & 3.3866 & 0.1175 \\ 0.000012 & & \end{array}$

C. Total $7 \quad 0.00014420$

Means for Oneway Anova

Level Number Mean Std Error Lower 95\% Upper 95\%

$\begin{array}{llllll}1 & 3 & 0.112056 & 0.00202 & 0.10686 & 0.11725\end{array}$

$\begin{array}{llllll}1 & 3 & 0.108158 & 0.00202 & 0.10296 & 0.11335 \\ 3 & 2 & 0.103774 & 0.00247 & 0.09741 & 0.11013\end{array}$

Std Error uses a pooled estimate of error variance
Oneway Analysis of Measurement By Block Glass ID=Batch 1, Oxide $=\mathrm{CuO}(\mathrm{wt} \%)$

Reference Value $=0.399$ wt $\%$

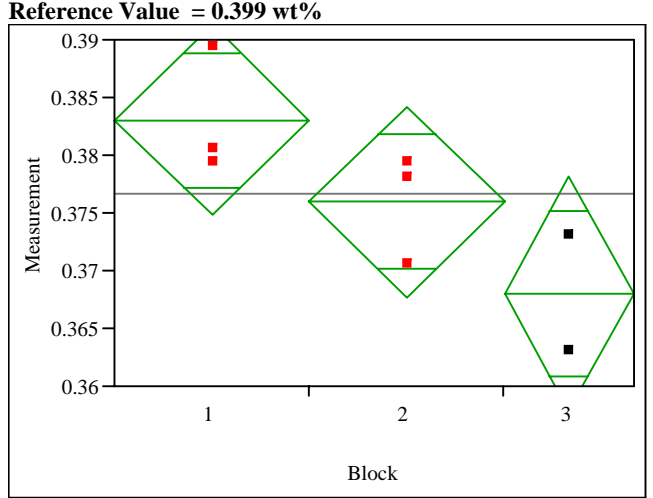

Oneway Anova

Summary of Fit

$\begin{array}{lr}\text { Rsquare } & 0.638418 \\ \text { Adj Rsquare } & 0.493785 \\ \text { Root Mean Square Error } & 0.005561 \\ \text { Mean of Response } & 0.376635 \\ \text { Observations (or Sum Wgts) } & 8\end{array}$

Analysis of Variance

Source DF Sum of Squares Mean Square F Ratio Prob $>$ F

$\begin{array}{lllllll}\text { Block } & 2 & 0.00027299 & 0.000136 & 4.4141 & 0.0786\end{array}$

$\begin{array}{llll}\text { Error } & 5 & 0.00015461 & 0.000031\end{array}$

C. Total $7 \quad 0.00042760$

Means for Oneway Anova

Level Number Mean Std Error Lower 95\% Upper 95\%

\begin{tabular}{llllll}
1 & 3 & 0.383051 & 0.00321 & 0.37480 & 0.39130 \\
\hline & 3 & 0.375957 & 0.0321 & 0.36770 & 0.38481
\end{tabular}

$\begin{array}{llllll}1 & 3 & 0.375957 & 0.00321 & 0.36770 & 0.38421 \\ 3 & 2 & 0.368029 & 0.00393 & 0.35792 & 0.37814\end{array}$

Std Error uses a pooled estimate of error variance 


\section{Figure A5. PSAL Measurements by Analytical Block for Samples of the Standard Glasses Prepared Using the LM Method}

Oneway Analysis of Measurement By Block Glass ID=Batch 1, Oxide $=\mathrm{La}_{2} \mathrm{O}_{3}(\mathrm{wt} \%)$

Reference Value $=0.0$ wt $\%$

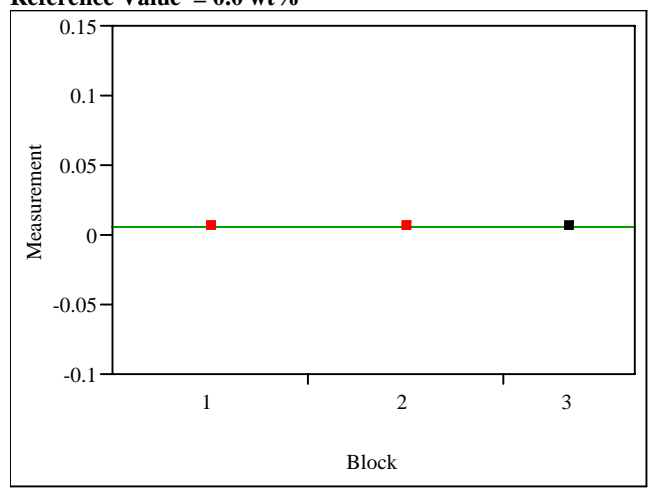

Oneway Anova

Summary of Fit

Rsquare

Adj Rsquare

Mean of Response

Observations (or Sum Wgts) 0.005864

Analysis of Variance

Source DF Sum of Squares Mean Square F Ratio Prob > F

Error

Sum of Squares Mean Square
0
0

C. Total 7

Means for Oneway Anova

Level Number Mean Std Error Lower 95\% Upper 95\%

$\begin{array}{llllll}1 & 3 & 0.005864 & 0 & 0.00586 & 0.00586\end{array}$

$\begin{array}{llllll}2 & 3 & 0.005864 & 0 & 0.00586 & 0.00586\end{array}$

Std Error uses a pooled estimate of error variance
Oneway Analysis of Measurement By Block Glass ID=Batch 1, Oxide $=\mathrm{MgO}(\mathrm{wt} \%)$

Reference Value $=1.419 \mathrm{wt} \%$

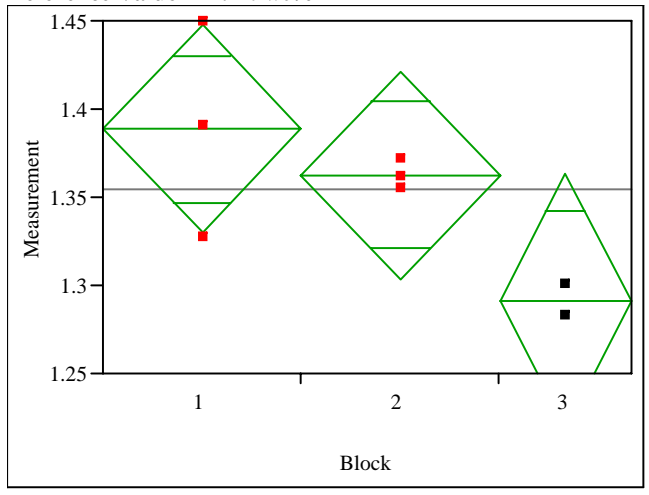

Oneway Anova

Summary of Fit

Rsquare

Adj Rsquare

0.4396

Reon of Response

Observations (or Sum Wgts)

0.03959

1.354417

Analysis of Variance

Source DF Sum of Squares Mean Square F Ratio Prob > F $\begin{array}{llllll}\text { Block } & 2 & 0.01174141 & 0.005871 & 3.7455 & 0.1014\end{array}$ \begin{tabular}{lll} 
Error & 5 & 0.00783692 \\
\hline
\end{tabular}

0.005871

C. Total $7 \quad 0.0195783$

Means for Oneway Anova

Level Number Mean Std Error Lower 95\% Upper 95\%

$\begin{array}{llllll}1 & 3 & 1.38855 & 0.02286 & 1.3298 & 1.4473 \\ 2 & 3 & 1.36257 & 0.02286 & 1.3038 & 1.4213\end{array}$

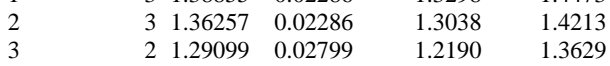

Std Error uses a pooled estimate of error variance
Oneway Analysis of Measurement By Block Glass ID=Batch 1, Oxide $=\mathrm{MnO}(\mathrm{wt} \%)$

Reference Value $=1.726$ wt $\%$

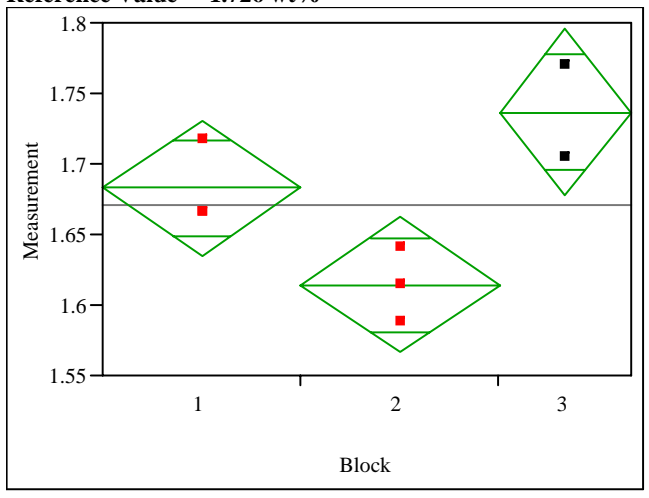

Oneway Anova

Summary of Fit

Rsquare

Rsquare

Adj Rsquare $\quad 0.783377$

$\begin{array}{ll}\text { Adj Rsquare } & 0.696727 \\ \text { Root Mean Square Error } \quad 0.032237\end{array}$

Mean of Respons

Observations (or Sum Wgts)

Analysis of Variance

Source DF Sum of Squares Mean Square F Ratio Prob $>$ F $\begin{array}{llllll}\text { Block } & 2 & 0.01879070 & 0.009395 & 9.0408 & 0.0218\end{array}$ $\begin{array}{llll}\text { Error } & 5 & 0.00519610 & 0.001039\end{array}$

$\begin{array}{lll}\text { C. Total } & 7 & 0.02398680\end{array}$

Means for Oneway Anova

Level Number Mean Std Error Lower 95\% Upper 95\%

$\begin{array}{llllll}1 & 3 & 1.68286 & 0.01861 & 1.6350 & 1.7307\end{array}$

$\begin{array}{llllll}2 & 3 & 1.61400 & 0.01861 & 1.5662 & 1.6618 \\ 3 & 2 & 1.73666 & 0.02279 & 1.6781 & 1.7953\end{array}$

Std Error uses a pooled estimate of error variance 


\section{Figure A5. PSAL Measurements by Analytical Block for Samples of the Standard Glasses Prepared Using the LM Method}

Oneway Analysis of Measurement By Block Glass ID=Batch 1, Oxide $=\mathrm{Na}_{2} \mathrm{O}(\mathrm{wt} \%)$

Reference Value $=9.003 \mathrm{wt} \%$

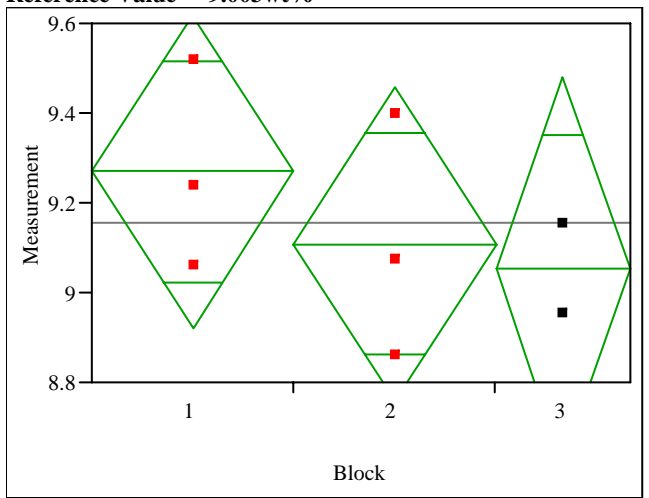

Oneway Anova
Summary of Fit

$\begin{array}{lr}\text { Rsquare } & 0.197061 \\ \text { Adj Rsquare } & -0.12411 \\ \text { Root Mean Square Error } & 0.2344 \\ \text { Mean of Response } & 9.154605 \\ \text { Observations (or Sum Wgts) } & 8\end{array}$

Source DF Sum of Squares Mean Square F Ratio Prob > F $\begin{array}{lllllll}\text { Block } & 2 & 0.06742213 & 0.033711 & 0.6136 & 0.5777\end{array}$ $\begin{array}{llll}\text { Error } & 5 & 0.27471584 & 0.054943\end{array}$

$\begin{array}{lll}\text { C. Total } & 7 & 0.34213797\end{array}$

Means for Oneway Anova

Level Number Mean Std Error Lower 95\% Upper 95\% $\begin{array}{llllll}1 & 3 & 9.26975 & 0.13533 & 8.9219 & 9.6176\end{array}$

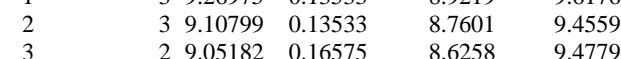

Std Error uses a pooled estimate of error variance
Oneway Analysis of Measurement By Block Glass ID=Batch 1, Oxide=NiO (wt\%)

Reference Value $=0.751 \mathrm{wt} \%$

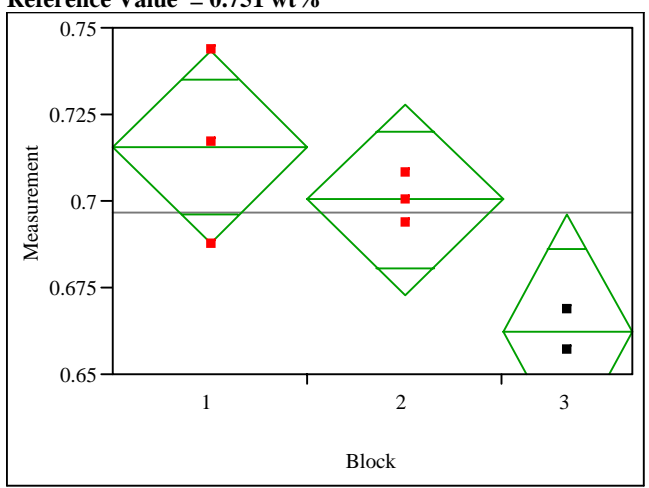

Oneway Anova

Summary of Fit

Rsquare

Adj Rsquare

Root Mean Square

Mean of Response

Observations (or Sum Wgts)

0.666913

0.533678

0.018614

Analysis of Variance

Source DF Sum of Squares Mean Square F Ratio Prob $>$ F $\begin{array}{llllll}\text { Block } & 2 & 0.00346851 & 0.001734 & 5.0055 & 0.0640\end{array}$

Error 50.00173233

C. Total 7

Means for Oneway Anova

Level Number Mean Std Error Lower 95\% Upper 95\%

\begin{tabular}{llllll}
1 & 3 & 0.715569 & 0.01075 & 0.68794 & 0.74319 \\
\hline & 3 & 0.700299 & 0.01075 & 0.67267 & 0.72792
\end{tabular}

$\begin{array}{llllll}3 & 0.700299 & 0.01075 & 0.67267 & 0.72792\end{array}$

Std Error uses a pooled estimate of error variance
Oneway Analysis of Measurement By Block Glass ID=Batch 1, Oxide $=\mathbf{P b O}$ (wt $\%)$

Reference $(w a)=0.0$ t $\%$

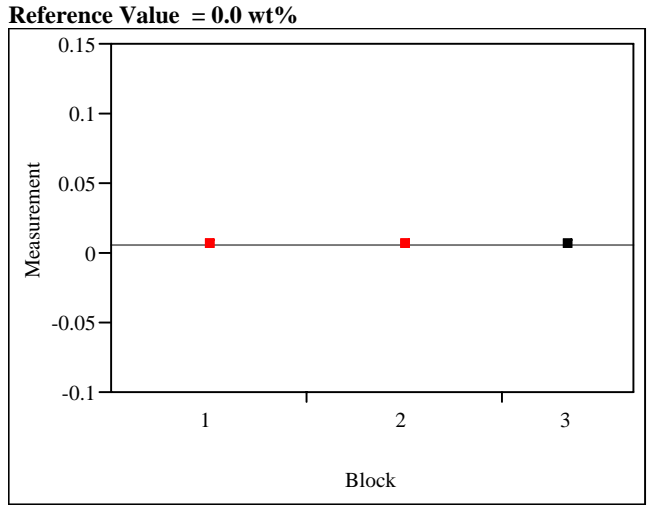

Oneway Anova

Summary of Fit

Rsquare

Adj Rsquare

A

0.005386

Analysis of Variance

Source DF Sum of Squares Mean Square F Ratio Prob > F $\begin{array}{llllll}\text { Block } 2 & 4.5139 \mathrm{e}-36 & 2.257 \mathrm{e}-36 & -2.5000 & 0.0000\end{array}$ $\begin{array}{lll}4 & -4.514 \mathrm{e}-36 & -9.03 \mathrm{e}-37\end{array}$

Means for Oneway Anova

Level Number Mean Std Error Lower 95\% Upper 95\% $1 \quad 3 \quad 0.005386$

$\begin{array}{lll}2 & 3 & 0.005386 \\ 3 & 2 & 0.005386\end{array}$

Std Error uses a pooled estimate of error variance 


\section{Figure A5. PSAL Measurements by Analytical Block for Samples of the Standard Glasses Prepared Using the LM Method}

Oneway Analysis of Measurement By Block Glass ID=Batch 1 Oxide $=\mathrm{SiO}_{2}(\mathrm{wt} \%)$

Reference Value $=\mathbf{5 0 . 2 2} \mathrm{wt} \%$

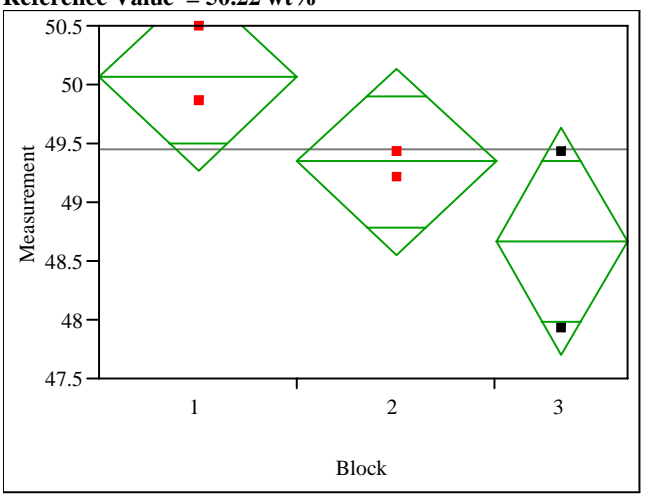

Oneway Anova

Summary of Fit

Adj Rsquare

0.623932

0.473504

Root Mean Square Error $\quad 0.534111$

Mean of Respons 49.44457
8

Analysis of Variance

Source DF Sum of Squares Mean Square F Ratio Prob $>$ F $\begin{array}{llllll}\text { Block } & 2 & 2.3664859 & 1.18324 & 4.1477 & 0.0867\end{array}$ $\begin{array}{lll}\text { Error } & 5 & 1.4263751\end{array}$ 0.28528

C. Total $7 \quad 3.792861$

Means for Oneway Anova

Level Number Mean Std Error Lower 95\% Upper 95\%

$\begin{array}{llllll}1 & 3 & 50.0596 & 0.30837 & 49.267 & 50.852\end{array}$

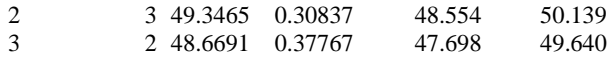

Std Error uses a pooled estimate of error variance
Oneway Analysis of Measurement By Block Glass ID=Batch 1, Oxide $=\mathrm{SO}_{4}(\mathrm{wt} \%)$

Reference Value $=0.0 \mathrm{wt} \%$

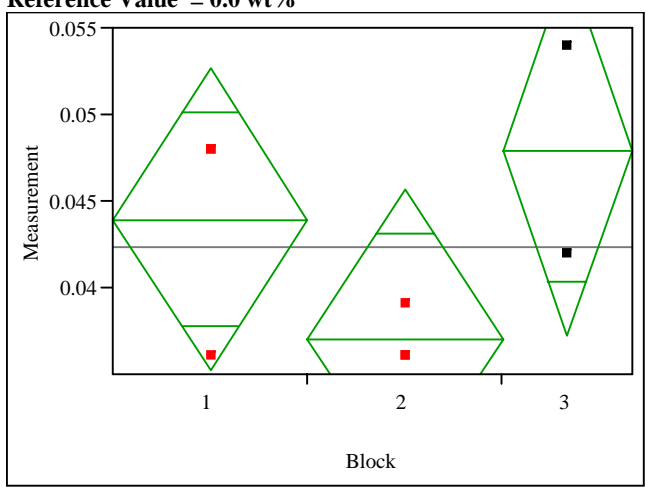

Oneway Anova

Summary of Fit

Rsquare

Adj Rsquare

Root Mean Square

Mean of Response

Observations (or Sum Wgts)

0.475706

0.265989

0.005891

0.042317

Analysis of Variance

Source DF Sum of Squares Mean Square F Ratio Prob > F

$\begin{array}{llllll}\text { Block } & 2 & 0.00015744 & 0.000079 & 2.2683 & 0.1990\end{array}$

$\begin{array}{llll}\text { Block } & 2 & 0.00015744 & 0.000079 \\ \text { Error } & 5 & 0.00017352 & 0.000035\end{array}$

$\begin{array}{lll}\text { C. Total } & 7 & 0.00033097\end{array}$

Means for Oneway Anova

Level Number Mean Std Error Lower 95\% Upper 95\%

$\begin{array}{llllll}1 & 3 & 0.043940 & 0.00340 & 0.03520 & 0.05268\end{array}$

$\begin{array}{llllll}2 & 3 & 0.036949 & 0.00340 & 0.02821 & 0.04569\end{array}$

Std Error uses a pooled estimate of error variance
Oneway Analysis of Measurement By Block Glass ID=Batch 1, Oxide $=\mathrm{TiO}_{2}(\mathrm{wt} \%)$

Reference Value $=0.677 \mathrm{wt} \%$

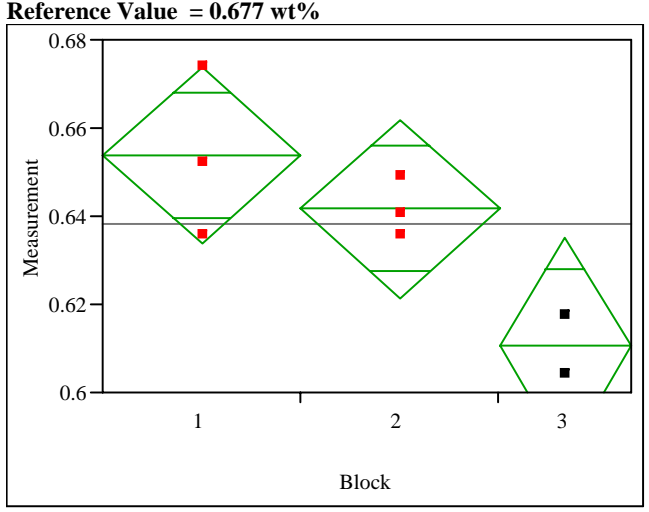

Oneway Anova

Summary of Fit

Rsquare

Adj Rsquare $\quad 0.71482$

$\begin{array}{ll}\text { Adj Rsquare } & 0.600747 \\ \text { Root Mean Square Error } & 0.013565\end{array}$

Mean of Response

Observations (or Sum Wgts)

Analysis of Variance

Source DF Sum of Squares Mean Square F Ratio Prob > F $\begin{array}{lllllll}\text { Block } & 2 & 0.00230600 & 0.001153 & 6.2664 & 0.0434\end{array}$ $\begin{array}{lll}\text { Error } & 5 & 0.00091999 \\ \text { C. Total } & 7 & 0.0032259\end{array}$ 0.000184

Means for Oneway Anova

Level Number Mean Std Error Lower 95\% Upper 95\% $\begin{array}{llllll}1 & 3 & 0.653856 & 0.00783 & 0.63372 & 0.67399\end{array}$ $\begin{array}{llllll}2 & 3 & 0.641624 & 0.00783 & 0.62149 & 0.66176\end{array}$ Std Error uses a pooled estimate of error variance 


\section{Figure A5. PSAL Measurements by Analytical Block for Samples of the Standard Glasses Prepared Using the LM Method}

Oneway Analysis of Measurement By Block Glass ID=Batch 1, Oxide $=\mathrm{U}_{3} \mathrm{O}_{8}(\mathrm{wt} \%)$

Reference Value $=0.0$ wt $\%$

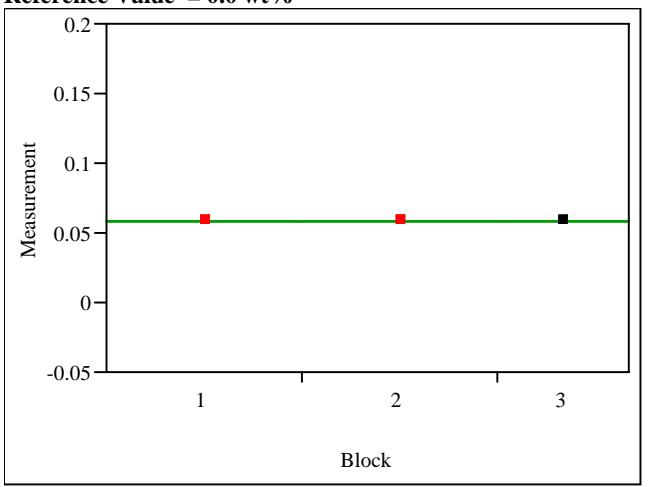

Oneway Anova

Summary of Fit

Rsquare

Adj Rsquare

Mean of Response

0
0.05896

Observations (or Sum Wgts) 8

Analysis of Variance

Source DF Sum of Squares Mean Square F Ratio Prob > F

Error

C Mean Square F Ratio Prob > F

C. Total 7

Means for Oneway Anova

Level Number Mean Std Error Lower 95\% Upper 95\%

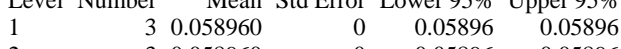

$\begin{array}{llllll}2 & 3 & 0.058960 & 0 & 0.05896 & 0.05896 \\ 3 & 2 & 0.058960 & 0 & 0.05896 & 0.05896\end{array}$

Std Error uses a pooled estimate of error variance
Oneway Analysis of Measurement By Block Glass ID=Batch 1, Oxide $=\mathrm{ZrO}_{2}$ ( $w$ t $\%$ )

Reference Value $=0.098$ wt $\%$

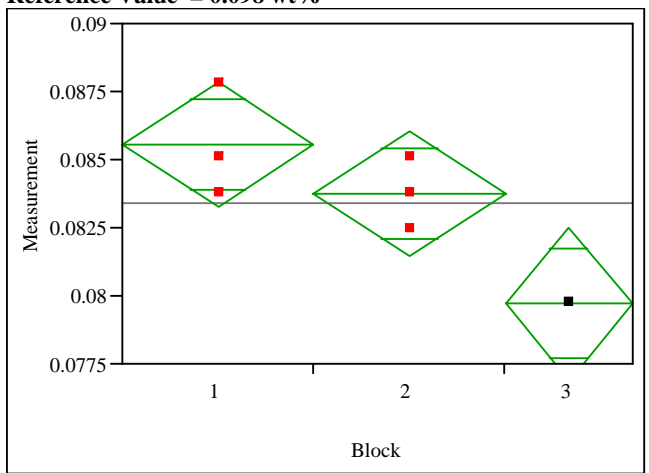

Oneway Anova

Oummary of Fit

$\begin{array}{lr}\text { Rsquare } & 0.774011 \\ \text { Adj Rsquare } & 0.683616 \\ \text { Root Mean Square Error } & 0.00156 \\ \text { Mean of Response } & 0.083412 \\ \text { Observations (or Sum Wgts) } & 8\end{array}$

Analysis of Variance

Source DF Sum of Squares Mean Square F Ratio Prob > F $\begin{array}{llllll}\text { Block } & 2 & 0.00004166 & 0.000021 & 8.5625 & 0.0243\end{array}$ $\begin{array}{llll}\text { Error } & 5 & 0.00001216 & 2.433 \mathrm{e}-6\end{array}$

C. Total $7 \quad 0.00005383$

Means for Oneway Anova

Level Number Mean Std Error Lower 95\% Upper 95\% $\begin{array}{llllll}1 & 3 & 0.085551 & 0.00090 & 0.08324 & 0.08787\end{array}$ $\begin{array}{llllll}1 & 3 & 0.083750 & 0.00090 & 0.08143 & 0.08606 \\ 3 & 2 & 0.079697 & 0.00110 & 0.07686 & 0.08253\end{array}$

Std Error uses a pooled estimate of error variance
Oneway Analysis of Measurement By Block Glass ID=Ustd Oxide $=\mathrm{Al}_{2} \mathrm{O}_{3}(\mathrm{wt} \%)$

Reference Value $=4.1 \mathrm{wt} \%$

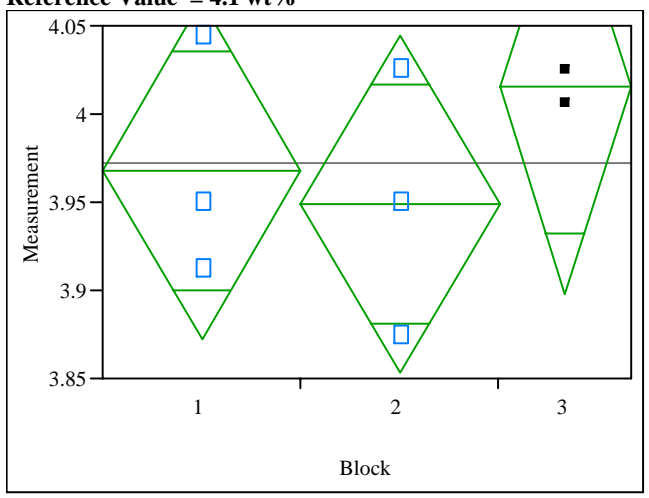

Oneway Anova

Summary of Fit

Adj Rsquare

0.204082

Root Mean Square Error $\quad 0.064631$

Mean of Respons

3.972674

Analysis of Variance

Source DF Sum of Squares Mean Square F Ratio Prob > F $\begin{array}{llllll}\text { Block } & 2 & 0.00535532 & 0.002678 & 0.6410 & 0.5652\end{array}$ $\begin{array}{llll}\text { Error } & 5 & 0.02088573 & 0.00417\end{array}$

C. Total $7 \quad 0.0262410$

Means for Oneway Anova

Level Number Mean Std Error Lower 95\% Upper 95\% $\begin{array}{llrrrr}1 & 3 & 3.96795 & 0.03731 & 3.8720 & 4.0639\end{array}$ $\begin{array}{llllll}2 & 3 & 3.94906 & 0.03731 & 3.8531 & 4.0450 \\ 3 & 2 & 4.01519 & 0.04570 & 3.8977 & 4.1327\end{array}$

Std Error uses a pooled estimate of error variance 


\section{Figure A5. PSAL Measurements by Analytical Block for Samples of the Standard Glasses Prepared Using the LM Method}

Oneway Analysis of Measurement By Block Glass ID=Ustd, Oxide=BaO $(\mathrm{wt} \%)$

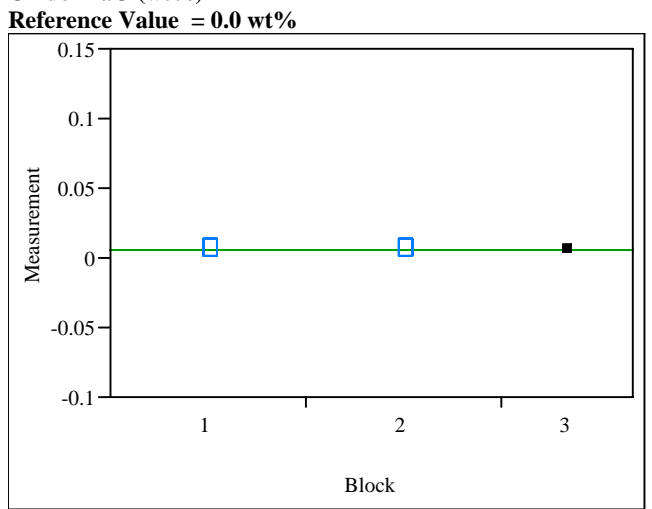

Oneway Anova

Summary of Fit

Rsquare

Adj Rsquare

$\begin{array}{lr}\text { Mean of Response } & 0.005583 \\ \text { Observations (or Sum Wgts) } & 8\end{array}$

Analysis of Variance

Source DF Sum of Squares Mean Square F Ratio Prob > F

Error

C. Total 7

0

Means for Oneway Anova

Level Number Mean Std Error Lower 95\% Upper 95\%

$\begin{array}{llllll}1 & 3 & 0.005583 & 0 & 0.00558 & 0.00558\end{array}$

$\begin{array}{llllll}2 & 3 & 0.005583 & 0 & 0.00558 & 0.00558\end{array}$

Std Error uses a pooled estimate of error variance
Oneway Analysis of Measurement By Block Glass ID=Ustd, Oxide $=\mathbf{C a O}(\mathrm{wt} \%)$

Reference Value $=1.301$ wt $\%$

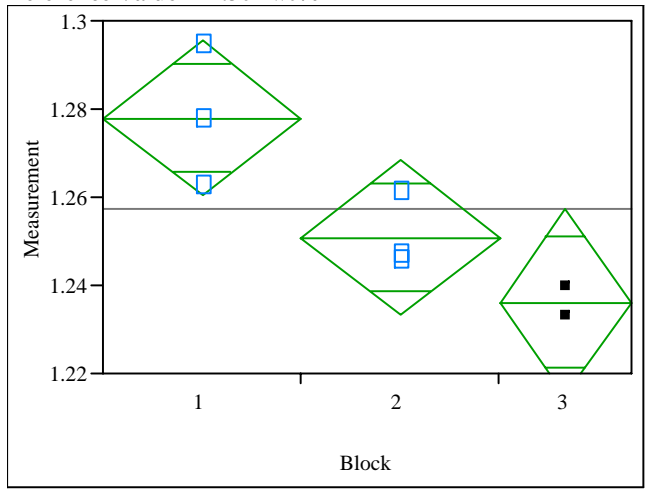

Oneway Anova

Summary of Fit

Rsquare

Rsquare

Root Mean Square Error $\quad 0.011726$

Mean of Response

1.257356

Analysis of Variance

Source DF Sum of Squares Mean Square F Ratio Prob > F $\begin{array}{llllll}\text { Block } & 2 & 0.00229197 & 0.001146 & 8.3344 & 0.0256\end{array}$ $\begin{array}{lll}\text { Error } & 5 & 0.00068750\end{array}$ 0.000138

Means for Oneway Anova

Level Number Mean Std Error Lower 95\% Upper 95\%

$\begin{array}{llllll}1 & 3 & 1.27794 & 0.00677 & 1.2605 & 1.2953\end{array}$

$\begin{array}{llllll}2 & 3 & 1.25088 & 0.00677 & 1.2335 & 1.2683 \\ 3 & 2 & & \end{array}$

Std Error uses a pooled estimate of error variance
Oneway Analysis of Measurement By Block Glass ID=Ustd, Oxide $=\mathrm{Ce}_{2} \mathrm{O}_{3}$ (wt $\%$ )

Reference Value $=0.0 \mathrm{wt} \%$

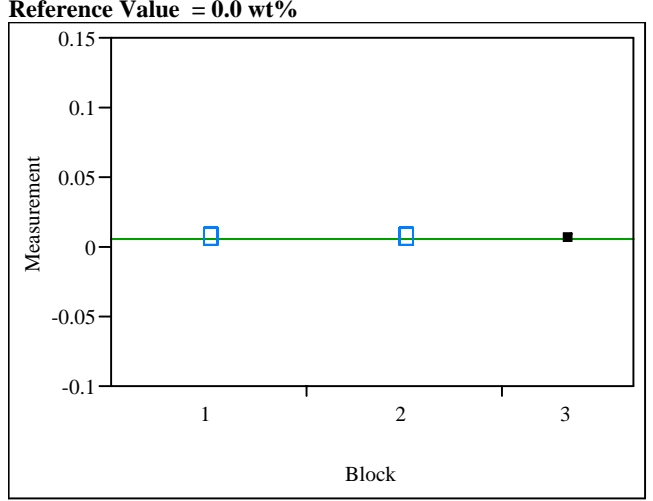

Oneway Anova

Summary of Fit

Rsquare

Adj Rsquare

Root Mean Square Error

$\begin{array}{lr}\text { Mean of Response } & 0.005857 \\ \text { Observations (or Sum Wgts) } & 8\end{array}$

Analysis of Variance

Source DF Sum of Squares Mean Square F Ratio Prob > F

$\begin{array}{llll}\text { Block } & 2 & 0 & 0 \\ \text { Error } & 5 & 0 & 0\end{array}$

$\begin{array}{lll}\text { Crror } & 5 & 0 \\ \text { Cotal } & 7 & 0\end{array}$

Means for Oneway Anova

Level Number Mean Std Error Lower 95\% Upper 95\% $\begin{array}{llllll}1 & 3 & 0.005857 & 0 & 0.00586 & 0.00586\end{array}$

$\begin{array}{llllll}1 & 3 & 0.005857 & 0 & 0.00586 & 0.00586 \\ 3 & 2 & 0.005857 & 0 & 0.00586 & 0.00586\end{array}$

Std Error uses a pooled estimate of error variance 


\section{Figure A5. PSAL Measurements by Analytical Block for Samples of the Standard Glasses Prepared Using the LM Method}

Oneway Analysis of Measurement By Block Glass ID=Ustd, Oxide $=\mathrm{Cr}_{2} \mathrm{O}_{3}(\mathrm{wt} \%)$

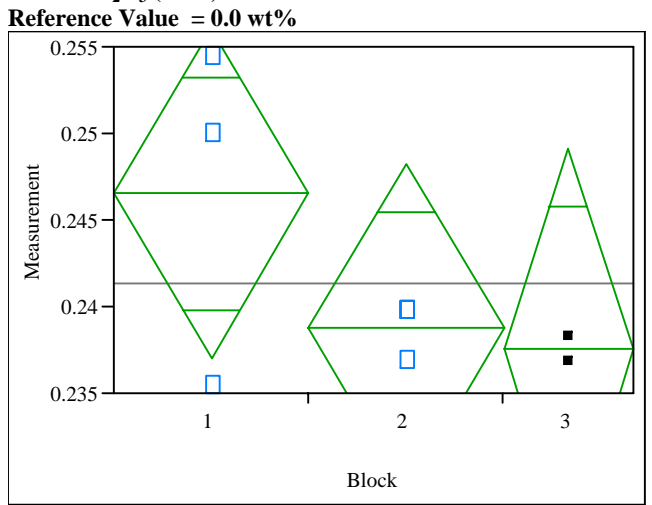

Oneway Anova

Summary of Fit

$\begin{array}{lr}\text { Rsquare } & 0.38911 \\ \text { Adj Rsquare } & 0.144754 \\ \text { Root Mean Square Error } & 0.006399 \\ \text { Mean of Response } & 0.241347 \\ \text { Observations (or Sum Wgts) } & 8\end{array}$

Analysis of Variance

Source DF Sum of Squares Mean Square F Ratio Prob $>$ F $\begin{array}{llllll}\text { Block } & 2 & 0.00013040 & 0.000065 & 1.5924 & 0.2917\end{array}$ $\begin{array}{llll}\text { Block } & 2 & 0.00013040 & 0.000065 \\ \text { Error } & 5 & 0.00020473 & 0.000041\end{array}$

$\begin{array}{lll}\text { C. Total } & 7 & 0.00033513\end{array}$

Means for Oneway Anova

Level Number Mean Std Error Lower 95\% Upper 95\%

$\begin{array}{llllll}1 & 3 & 0.246523 & 0.00369 & 0.23703 & 0.25602\end{array}$

$\begin{array}{lllll}3 & 0.238728 & 0.00369 & 0.22923 & 0.24822\end{array}$

Std Error uses a pooled estimate of error variance
Oneway Analysis of Measurement By Block Glass ID=Ustd, Oxide $=\mathrm{CuO}(\mathrm{wt} \%)$

Reference Value $=0.0 \mathrm{wt} \%$

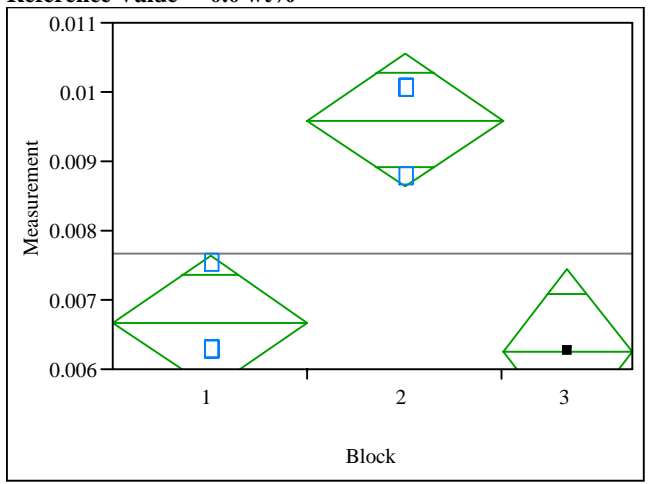

Oneway Anova

Summary of Fit

Rsquare

Adj Rsquare

Root Mean Square

Mean of Response

Observations (or Sum Wgs)

0.89644

0.855016

0.000646

Analysis of Variance

Source DF Sum of Squares Mean Square F Ratio Prob > F

$\begin{array}{lllllll}\text { Block } & 2 & 0.00001809 & 9.0429 \mathrm{e}-6 & 21.6406 & 0.0035\end{array}$

Error $5-0.00000209$

C. Total $7-0.00002018$

Means for Oneway Anova

Level Number Mean Std Error Lower 95\% Upper 95\%

$\begin{array}{llllll}1 & 3 & 0.006676 & 0.00037 & 0.00572 & 0.00764\end{array}$

$\begin{array}{llllll}2 & 3 & 0.009597 & 0.00037 & 0.00864 & 0.01056\end{array}$

Std Error uses a pooled estimate of error variance
Oneway Analysis of Measurement By Block Glass ID=Ustd, Oxide $=\mathrm{La}_{2} \mathrm{O}_{3}$ (wt \%)

Reference Value $=0.0$ wto

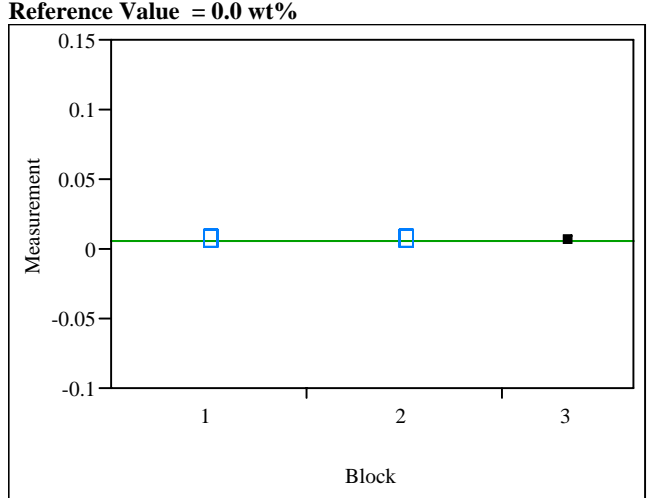

Oneway Anova

Summary of Fit

Rsquare

Adj Rsquare

Mean Square Error

0.005864

Observations (or Sum Wgts) 8

Analysis of Variance

Source DF Sum of Squares Mean Square F Ratio Prob > F

$\begin{array}{llll}\text { Error } & 5 & 0 & 0 \\ \text { C. To } & 7 & 0 & 0\end{array}$

7

Means for Oneway Anova

Level Number Mean Std Error Lower 95\% Upper 95\% $\begin{array}{rrrrrr}1 & 3 & 0.005864 & 0 & 0.00586 & 0.00586\end{array}$

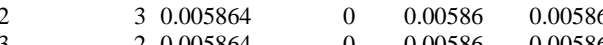

Std Error uses a pooled estimate of error variance 


\section{Figure A5. PSAL Measurements by Analytical Block for Samples of the Standard Glasses Prepared Using the LM Method}

Oneway Analysis of Measurement By Block Glass ID=Ustd, Oxide=MgO (wt \%)

Reference Value $=1.21$ wt\%

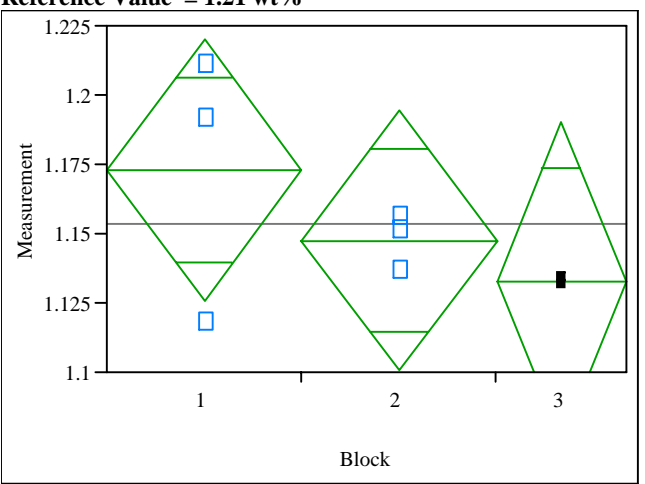

Oneway Anova

Summary of Fit

$\begin{array}{lr}\text { Rsquare } & 0.297269 \\ \text { Adj Rsquare } & 0.016177 \\ \text { Root Mean Square Error } & 0.031627 \\ \text { Mean of Response } & 1.153348 \\ \text { Observations (or Sum Wgts) } & 8\end{array}$

Analysis of Variance

Source DF Sum of Squares Mean Square F Ratio Prob > F

$\begin{array}{llllll}\text { Block } & 2 & 0.00211564 & 0.001058 & 1.0576 & 0.4140\end{array}$

$\begin{array}{llll}\text { Block } & 2 & 0.00211564 & 0.00105 \\ \text { Error } & 5 & 0.00500126 & 0.001000\end{array}$

$\begin{array}{lll}\text { C. Total } & 7 & 0.00711689\end{array}$

Means for Oneway Anova

Level Number Mean Std Error Lower 95\% Upper 95\%

$\begin{array}{lllllr}1 & 3 & 1.17297 & 0.01826 & 1.1260 & 1.2199\end{array}$

$\begin{array}{llllll}2 & 3 & 1.14754 & 0.01826 & 1.1006 & 1.1945\end{array}$

Std Error uses a pooled estimate of error variance
Oneway Analysis of Measurement By Block Glass ID=Ustd, Oxide $=\mathrm{MnO}(\mathrm{wt} \%)$

Reference Value = $2.892 \mathrm{wt} \%$

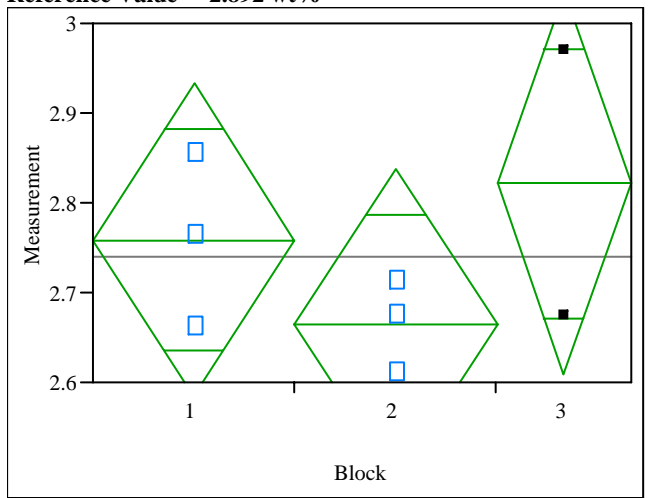

Oneway Anova

Summary of Fit

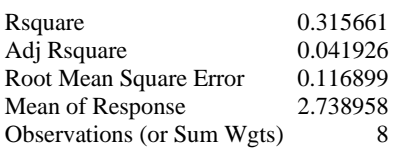

Observations (or Sum Wgts)

Analysis of Variance

Source DF Sum of Squares Mean Square F Ratio Prob > F $\begin{array}{llllll}\text { Block } & 2 & 0.03151698 & 0.015758 & 1.1532 & 0.3874\end{array}$ $\begin{array}{lll}\text { Error } & 5 & 0.06832731\end{array}$ 0.013665

C. Total 70.09984429

Means for Oneway Anova $\begin{array}{lllll}3 & 2.75886 & 0.06749 & 2.5854 & 2.9324\end{array}$ $\begin{array}{llllll}1 & 3 & 2.75618 & 0.06749 & 2.4907 & 2.8377\end{array}$

Std Error uses a pooled estimate of error variance
Oneway Analysis of Measurement By Block Glass ID=Ustd, Oxide $=\mathrm{Na}_{2} \mathrm{O}(\mathrm{wt} \%)$

Reference Value $=11.795 \mathrm{wt} \%$

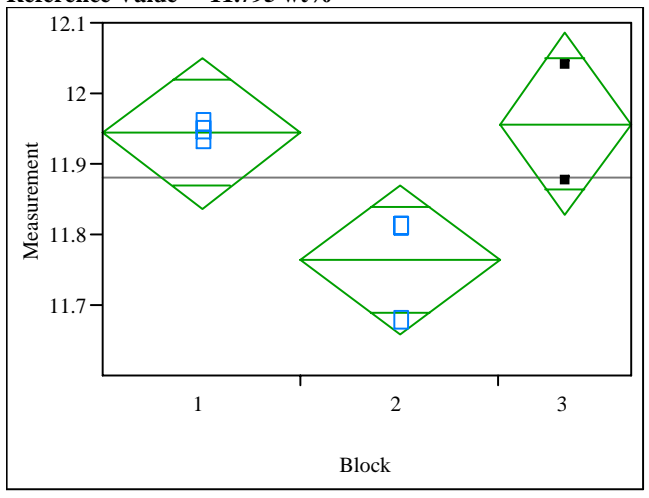

Oneway Anova

Summary of Fit

Rsquare

Adj Rsquare $\quad 0.716112$

Root Mean Square Enor $\quad 0.602556$

Roor Mean Syare Error 0.071499

Mean of Response

11.87925

Analysis of Variance

Source DF Sum of Squares Mean Square F Ratio Prob > F $\begin{array}{lllrlr}\text { Block } & 2 & 0.06447691 & 0.032238 & 6.3063 & 0.0429\end{array}$ $\begin{array}{lll}\text { Error } & 5 & 0.02556060\end{array}$

$\begin{array}{lll}\text { C. Total } & 7 & 0.0900375\end{array}$

$\begin{array}{lll}0.032238 & 6.3063 & 0.0429 \\ 0.005112 & & \end{array}$

Means for Oneway Anova

Level Number Mean Std Error Lower 95\% Upper 95\% $\begin{array}{llllrr}1 & 3 & 11.9433 & 0.04128 & 11.837 & 12.049\end{array}$ $\begin{array}{llllll}2 & 3 & 11.7635 & 0.04128 & 11.657 & 11.870 \\ 3 & 2 & 11.9568 & 0.05056 & 11.827 & 12.087\end{array}$

Std Error uses a pooled estimate of error variance 


\section{Figure A5. PSAL Measurements by Analytical Block for Samples of the Standard Glasses Prepared Using the LM Method}

Oneway Analysis of Measurement By Block Glass ID=Ustd, Oxide=NiO (wt\%)

Reference Value $=1.12$ wt \%

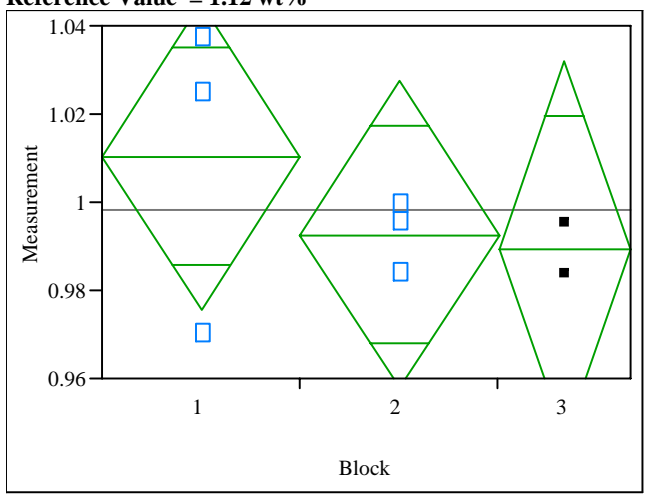

Oneway Anova

Summary of Fit

Rsquare

Adj Rsquare

Root Mean Square Error $\quad 0.023495$

Mean of Respons

0.998435

Analysis of Variance

Source DF Sum of Squares Mean Square F Ratio Prob $>$ F

$\begin{array}{lrrrrr}\text { Solock } & 2 & 0.00069527 & 0.000348 & 0.6298 & 0.5703 \\ \text { Brror } & 5 & 0.00276002 & 0.000552 & & \end{array}$

$\begin{array}{lll}\text { Error } & 5 & 0.00276002\end{array}$

C. Total $7 \quad 0.00345529$

Means for Oneway Anova

Level Number Mean Std Error Lower 95\% Upper 95\%

$\begin{array}{llllll}1 & 3 & 1.01037 & 0.01356 & 0.97550 & 1.0452\end{array}$

$\begin{array}{llllll}2 & 3 & 0.99255 & 0.01356 & 0.95768 & 1.0274 \\ 3 & 2 & 0.98937 & 0.01661 & 0.94666 & 1.0321\end{array}$

Std Error uses a pooled estimate of error variance
Oneway Analysis of Measurement By Block Glass ID=Ustd, Oxide $=\mathbf{P b O}(\mathrm{wt} \%)$

Reference Value $=0.0$ wt $\%$

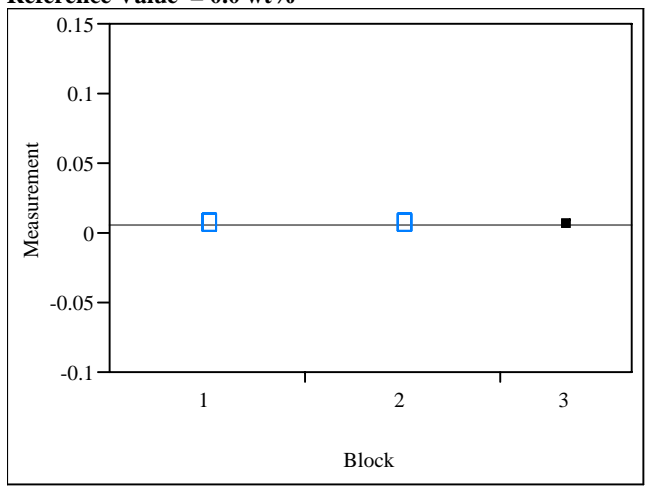

Oneway Anova

Summary of Fit

Rsquare

Adj Rsquare

A Mean Square Error

0.005386

Analysis of Variance

Source DF Sum of Squares Mean Square F Ratio Prob $>$ F

$\begin{array}{lllll}\text { Block } 2 & 4.5139 \mathrm{e}-36 & 2.257 \mathrm{e}-36 & -2.5000 & 0.0000\end{array}$

$\begin{array}{llll}\text { Error } \quad 5 & -4.514 \mathrm{e}-36 & -9.03 \mathrm{e}-37\end{array}$

$-4.514-36$

Means for Oneway Anova

Level Number Mean Std Error Lower 95\% Upper 95\% $1 \quad 3 \quad 0.005386$

$\begin{array}{lll}1 & 3 & 0.005386 \\ 3 & 2 & 0.005386\end{array}$

Std Error uses a pooled estimate of error variance
Oneway Analysis of Measurement By Block Glass ID=Ustd Oxide $=\mathrm{SiO}_{2}(\mathrm{wt} \%)$

Reference Value $=45.353 \mathrm{wt} \%$

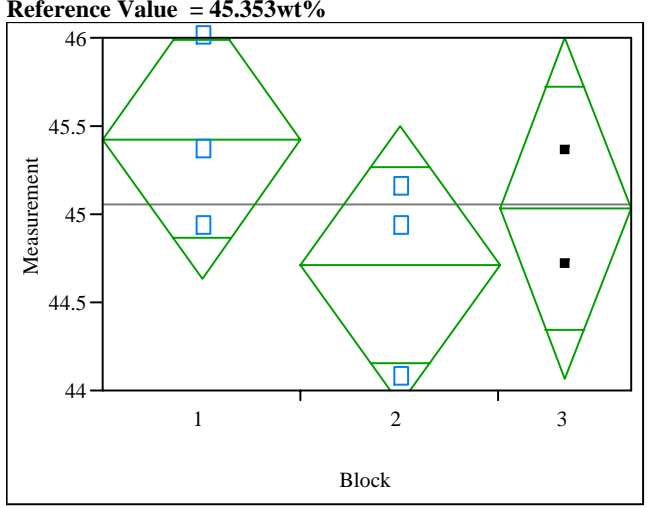

Oneway Anova

Summary of Fit

Rsquare

Adj Rsquare 0.348999

Root Mes Square Enor $\quad 0.088599$

Root Mean Square Er

Observations (or Sum 45.05901

Analysis of Variance

Source DF Sum of Squares Mean Square F Ratio Prob > F $\begin{array}{llllll}\text { Block } & 2 & 0.7646743 & 0.382337 & 1.3402 & 0.3419\end{array}$ $\begin{array}{llll}\text { Error } & 5 & 1.4263751 & 0.285275\end{array}$

C. Total $7 \quad 2.191049$

Means for Oneway Anova

Level Number Mean Std Error Lower 95\% Upper 95\% $\begin{array}{rrrrrr}1 & 3 & 45.4245 & 0.30837 & 44.632 & 46.217\end{array}$ $\begin{array}{llllll}2 & 3 & 44.7114 & 0.30837 & 43.919 & 45.504 \\ 3 & 2 & 45.0323 & 0.37767 & 44.061 & 46.003\end{array}$

Std Error uses a pooled estimate of error variance 


\section{Figure A5. PSAL Measurements by Analytical Block for Samples of the Standard Glasses Prepared Using the LM Method}

Oneway Analysis of Measurement By Block Glass ID=Ustd, Oxide $=\mathrm{SO}_{4}(\mathrm{wt} \%)$

Reference Value $=0.0$ wt $\%$

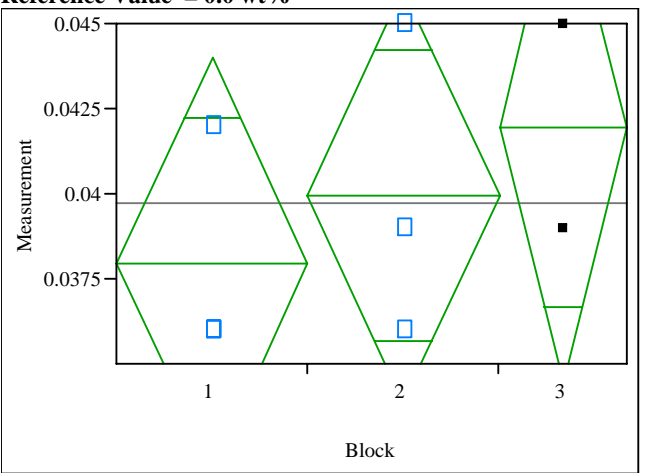

Oneway Anova

Summary of Fit

$\begin{array}{lr}\text { Rsquare } & 0.188406 \\ \text { Adj Rsquare } & -0.13623 \\ \text { Root Mean Square Error } & 0.004093 \\ \text { Mean of Response } & 0.039696 \\ \text { Observations (or Sum Wgts) } & 8\end{array}$

Analysis of Variance

Source DF Sum of Squares Mean Square F Ratio Prob > F $\begin{array}{lllllll}\text { Block } & 2 & 0.00001945 & 9.723 \mathrm{e}-6 & 0.5804 & 0.5934\end{array}$

$\begin{array}{lll}\text { Error } & 5 & 0.00008377\end{array}$ 0.000017

C. Total $7 \quad 0.0001032$

Level Number Mean Std Error Lower 95\% Upper 95\%

$\begin{array}{lllll}3 & 0.037948 & 0.00236 & 0.03187 & 0.04402\end{array}$

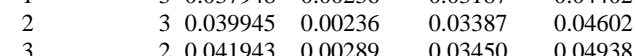

Std Error uses a pooled estimate of error variance
Oneway Analysis of Measurement By Block Glass ID=Ustd, Oxide $=\mathrm{TiO}_{2}(\mathrm{wt} \%)$

Reference Value = $1.049 \mathrm{wt} \%$

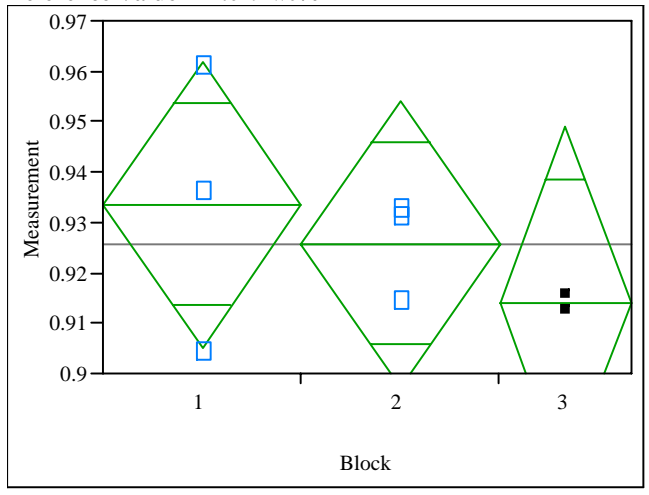

\section{Oneway Anova}

Summary of Fit

Adj Rsquare

Root Mean Square

Mean of Response

0.199187

$-0.12114$ 0.92574

Observations (or Sum Wgts) 8

Analysis of Variance

Source DF Sum of Squares Mean Square F Ratio Prob > F $\begin{array}{llllll}\text { Block } & 2 & 0.00045443 & 0.000227 & 0.6218 & 0.5739\end{array}$ $\begin{array}{llll}\text { Error } & 5 & 0.00182699 & 0.000365\end{array}$

C. Total $\quad 7 \quad 0.0022814$

Means for Oneway Anova

Level Number Mean Std Error Lower 95\% Upper 95\%

$\begin{array}{llllll}1 & 3 & 0.933524 & 0.01104 & 0.90515 & 0.96189\end{array}$

$\begin{array}{llllll}2 & 3 & 0.925740 & 0.01104 & 0.89737 & 0.95411 \\ 3 & 2 & 0.914064 & 0.01352 & 0.87932 & 0.94881\end{array}$

Std Error uses a pooled estimate of error variance
Oneway Analysis of Measurement By Block Glass ID=Ustd, Oxide $=\mathrm{U}_{3} \mathrm{O}_{8}(\mathrm{wt} \%)$

Reference Value $=2.406 \mathrm{wt} \%$
Re

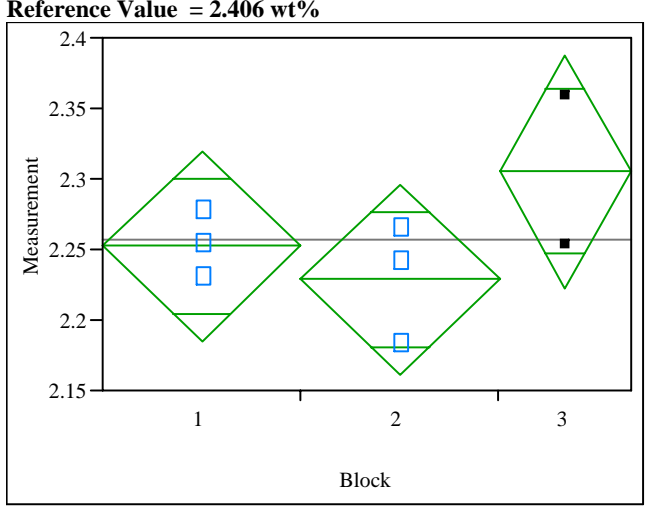

Oneway Anova

Summary of Fit

Rsquare

Adj Rsquare

0.408143

Root Mean Square Error $\quad \begin{array}{r}0.045518 \\ \hline\end{array}$

Mean of Respons

2.256694

Analysis of Variance

Source DF Sum of Squares Mean Square F Ratio Prob > F $\begin{array}{llllll}\text { Block } & 2 & 0.00714376 & 0.003572 & 1.7240 & 0.2695\end{array}$ $\begin{array}{llll}\text { Error } & 5 & 0.01035932 & 0.002072\end{array}$

C. Total $7 \quad 0.0175030$

Means for Oneway Anova

Level Number Mean Std Error Lower 95\% Upper 95\% $\begin{array}{llllll}1 & 3 & 2.25227 & 0.02628 & 2.1847 & 2.3198\end{array}$ $\begin{array}{llllll}2 & 3 & 2.22869 & 0.02628 & 2.1611 & 2.2962 \\ 3 & 2 & 2.30534 & 0.03219 & 2.2226 & 2.3881\end{array}$

Std Error uses a pooled estimate of error variance 


\section{Figure A5. PSAL Measurements by Analytical Block for Samples of the Standard Glasses Prepared Using the LM Method}

Oneway Analysis of Measurement By Block Glass ID=Ustd, Oxide $=\mathrm{ZrO}_{2}(\mathrm{wt} \%)$

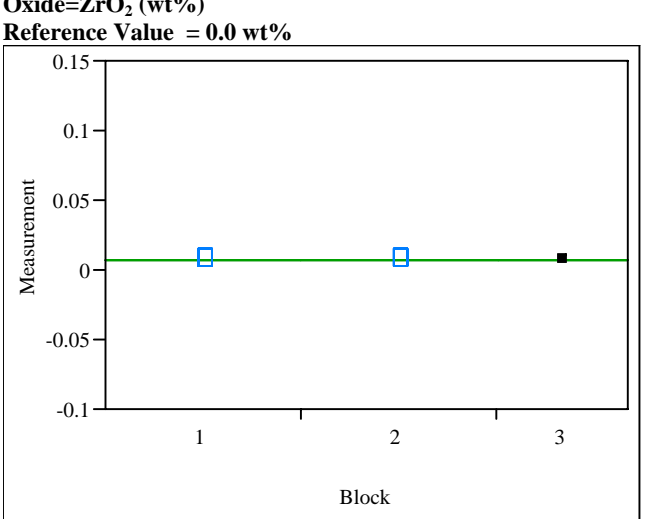

Oneway Anova

Summary of Fit

Rsquare

Adj Rsquare

$\begin{array}{lr}\text { Root Mean Square Error } & 0 \\ \text { Mean of Response } & 0.006754\end{array}$

$\begin{array}{lr}\text { Mean of Response } & 0.006754 \\ \text { Observations (or Sum Wgts) } & 8\end{array}$

Analysis of Variance

Source DF Sum of Squares Mean Square F Ratio Prob > F

Error $\begin{array}{rr}0 & \text { Mean Square } \\ 0 & 0 \\ 0 & 0\end{array}$

C. Total 7

Means for Oneway Anova

Level Number Mean Std Error Lower 95\% Upper 95\%

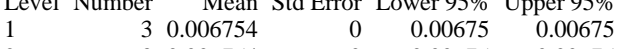

$\begin{array}{lllll}3 & 0.006754 & 0 & 0.00675 & 0.00675\end{array}$

Std Error uses a pooled estimate of error variance 
Figure A6. PSAL Measurements by Analytical Block for Samples of the Standard Glasses Prepared Using the PF Method

Oneway Analysis of Measurement By Block Glass ID=Batch 1, Oxide $=\mathbf{B}_{2} \mathrm{O}_{3}(\mathrm{wt} \%)$

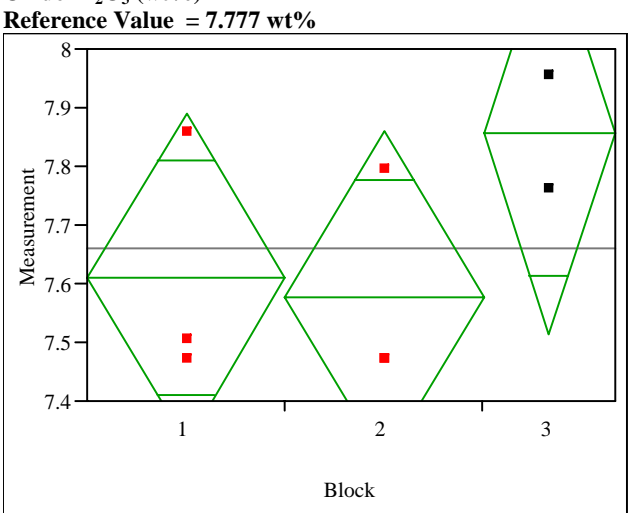

Oneway Anova

Summary of Fit

$\begin{array}{lr}\text { Rsquare } & 0.36941 \\ \text { Adj Rsquare } & 0.117174 \\ \text { Root Mean Square Error } & 0.189583 \\ \text { Mean of Response } & 7.659337 \\ \text { Observations (or Sum Wgts) } & 8\end{array}$

Analysis of Variance

Source DF Sum of Squares Mean Square F Ratio Prob $>$ F $\begin{array}{llllll}\text { Block } & 2 & 0.10527592 & 0.052638 & 1.4645 & 0.3158\end{array}$ $\begin{array}{llll}\text { Error } & 5 & 0.17970777 & 0.035942\end{array}$

C. Total $\quad 7 \quad 0.28498369$

Means for Oneway Anova

Level Number Mean Std Error Lower 95\% Upper 95\% $\begin{array}{llllll}1 & 3 & 7.60970 & 0.10946 & 7.3283 & 7.8911\end{array}$ $\begin{array}{llllll}2 & 3 & 7.57750 & 0.10946 & 7.2961 & 7.8589 \\ 3 & 2 & 7.85656 & 0.13406 & 7.5120 & 8.2012\end{array}$

Std Error uses a pooled estimate of error variance
Oneway Analysis of Measurement By Block Glass ID=Batch 1

Oxide $=\mathrm{Fe}_{2} \mathrm{O}_{3}(w t \%)$
Reference Value $=12.839 \mathrm{wt} \%$

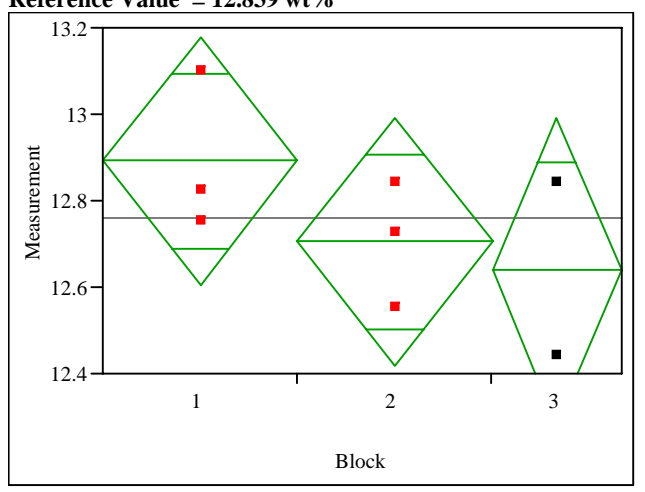

\section{Oneway Anova}

Summary of Fit

$\begin{array}{lr}\text { Rsquare } & 0.324913 \\ \text { Adj Rsquare } & 0.054878 \\ \text { Root Mean Square Error } & 0.193441 \\ \text { Mean of Response } & 12.75829 \\ \text { Observations (or Sum Wgts) } & 8\end{array}$

Analysis of Variance

Source DF Sum of Squares Mean Square F Ratio Prob > F

$\begin{array}{llllll}\text { Block } & 2 & 0.09004857 & 0.045024 & 1.2032 & 0.3745\end{array}$

$\begin{array}{llll}\text { Error } & 5 & 0.18709799 & 0.037420\end{array}$

$\begin{array}{lll}\text { C. Total } & 7 & 0.27714656\end{array}$

Means for Oneway Anova

Level Number Mean Std Error Lower 95\% Upper 95\%

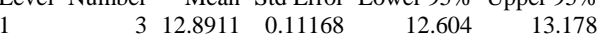

$\begin{array}{llllll}2 & 3 & 12.7053 & 0.11168 & 12.418 & 12.992 \\ 3 & 2 & 12.6385 & 0.13678 & 12.287 & 12.990\end{array}$

Std Error uses a pooled estimate of error variance
Oneway Analysis of Measurement By Block Glass ID=Batch 1, Oxide $=\mathrm{Li}_{2} \mathbf{O}$ (wt\%)

Reference Value $=4.429 \mathrm{wt} \%$

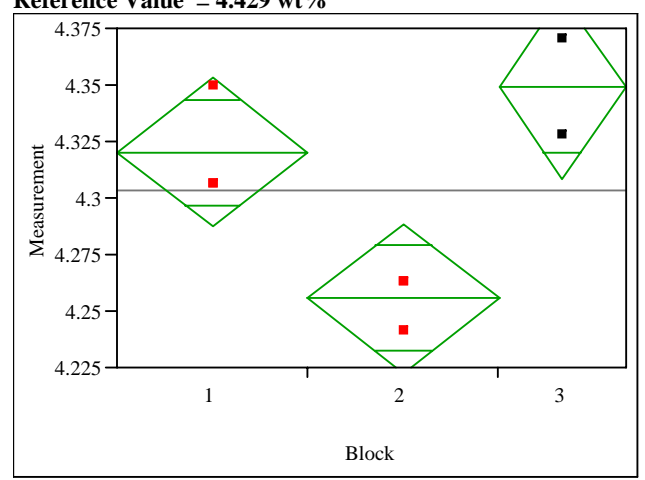

Oneway Anova

Summary of Fit

Rsquare

$\begin{array}{ll} & 0.82726 \\ \text { Adj Rsquare } & 0.758165\end{array}$

Adj Rsquare $\quad 0.758165$

Root Mean Square Enor $\quad 0.022235$

$\begin{array}{lr}\text { Mean of Response } & 4.303109 \\ \text { Observations (or Sum Wgts) } & 8\end{array}$

Analysis of Variance

Source DF Sum of Squares Mean Square F Ratio Prob $>$ F $\begin{array}{lrrrrr}\text { Block } & 2 & 0.01183851 & 0.005919 & 11.9727 & 0.0124\end{array}$ $\begin{array}{llll} & 5 & 0.00247199 & 0.000494\end{array}$

$\begin{array}{ll}5 & 0.01431050\end{array}$

Means for Oneway Anova

Level Number Mean Std Error Lower 95\% Upper 95\%

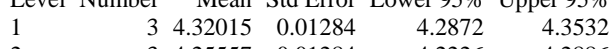

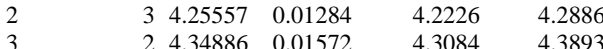

Std Error uses a pooled estimate of error variance 
Figure A6. PSAL Measurements by Analytical Block for Samples of the Standard Glasses Prepared Using the PF Method

Oneway Analysis of Measurement By Block Glass ID=Ustd, Oxide $=\mathbf{B}_{2} \mathrm{O}_{3}(\mathrm{wt} \%)$

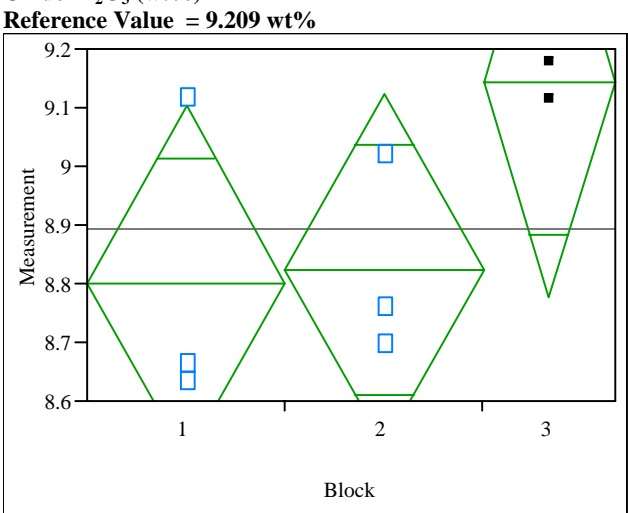

Oneway Anova

Summary of Fit

$\begin{array}{lr}\text { Rsquare } & 0.447381 \\ \text { Adj Rsquare } & 0.226333 \\ \text { Root Mean Square Error } & 0.202964 \\ \text { Mean of Response } & 8.894974 \\ \text { Observations (or Sum Wgts) } & 8\end{array}$

Analysis of Variance

Source DF Sum of Squares Mean Square F Ratio Prob $>$ F $\begin{array}{llllll}\text { Block } & 2 & 0.16674808 & 0.083374 & 2.0239 & 0.2270\end{array}$ $\begin{array}{llll}\text { Error } & 5 & 0.20597275 & 0.041195\end{array}$

C. Total $7 \quad 0.37272083$

Means for Oneway Anova

Level Number Mean Std Error Lower 95\% Upper 95\%

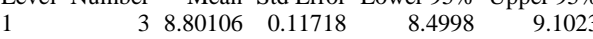

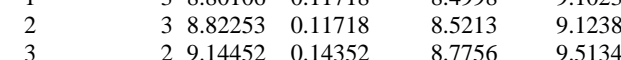

Std Error uses a pooled estimate of error variance
Oneway Analysis of Measurement By Block Glass ID=Ustd Oxide $=\mathrm{Fe}_{2} \mathrm{O}_{3}$ (wt \%)

Reference Value $=13.196 \mathrm{wt} \%$

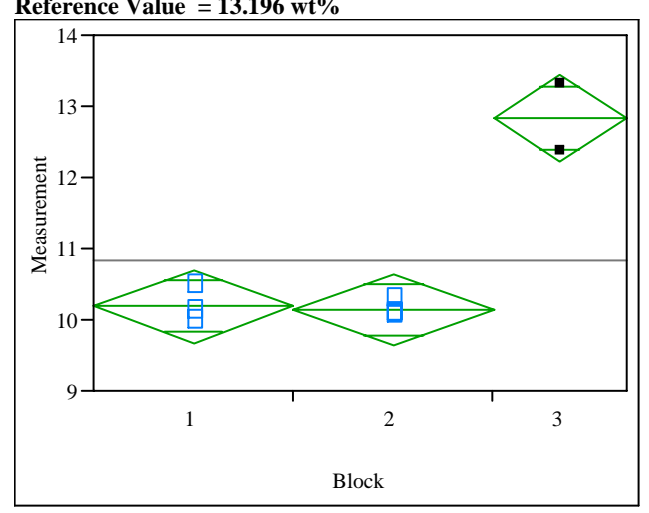

\section{Oneway Anova}

Summary of Fi

Rsquare

Adj Rsquare

Root Mean Square

Observatiosponse

0.948237

0.927532

0.341885

Observations (or Sum Wgts)

8

Analysis of Variance

Source DF Sum of Squares Mean Square F Ratio Prob $>$ F

$\begin{array}{llllll}\text { Block } & 2 & 10.706020 & 5.35301 & 45.7972 & 0.0006\end{array}$

$\begin{array}{llrr}\text { Error } & 5 & 0.584426 & 0.11689\end{array}$

C. Total $7 \quad 11.290445$

Means for Oneway Anova
Level Number Mean Std Error Lower 95\% Upper 95\%

\begin{tabular}{llrrrr}
1 & 3 & 10.1842 & 0.19739 & 9.677 & 10.692 \\
\hline & 3 & 10.1366 & 0.19739 & 9.629 & 1.64
\end{tabular}

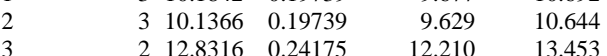

Std Error uses a pooled estimate of error variance
Oneway Analysis of Measurement By Block Glass ID=Ustd, Oxide $=\mathrm{Li}_{2} \mathrm{O}$ (wt\%)

Reference Value $=3.057 \mathrm{wt} \%$

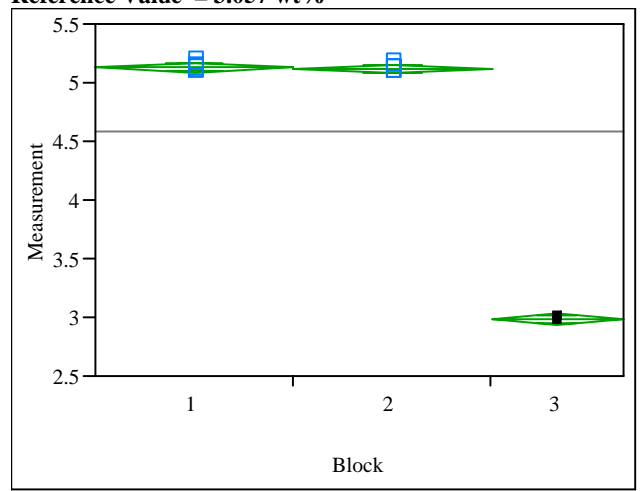

Oneway Anova

Summary of Fit

Rsquare $\quad 0.999473$

Adj Rsquare Root Mean Square Error $\quad 0.999262$

Mean of Response 4.588368

Observations (or Sum Wgts)

Analysis of Variance

Source DF Sum of Squares Mean Square F Ratio Prob $>$ F $\begin{array}{llllll}\text { Block } & 2 & 6.8834257 & 3.44171 & 4739.694 & <.0001\end{array}$

$\begin{array}{llll}\text { Error } & 5 & 0.0036307 & 0.00073\end{array}$

C. Total $7 \quad 6.8870565$

Means for Oneway Anova

Level Number Mean Std Error Lower 95\% Upper 95\% $\begin{array}{llllll}1 & 3 & 5.13108 & 0.01556 & 5.0911 & 5.1711\end{array}$

$\begin{array}{llllll}2 & 3 & 5.11673 & 0.01556 & 5.0767 & 5.1567 \\ 3 & 2 & 2.98177 & 0.01905 & 2.9328 & 3.0307\end{array}$

Std Error uses a pooled estimate of error variance 
Figure A7. Oxide Measurements by Lab ID within Glass ID Including Auxiliary Measurements for LM Preps

Variability Gage $\mathrm{Oxide}=\mathrm{Al}_{2} \mathrm{O}_{3}(\mathrm{wt} \%)$

Variability Chart for Measured

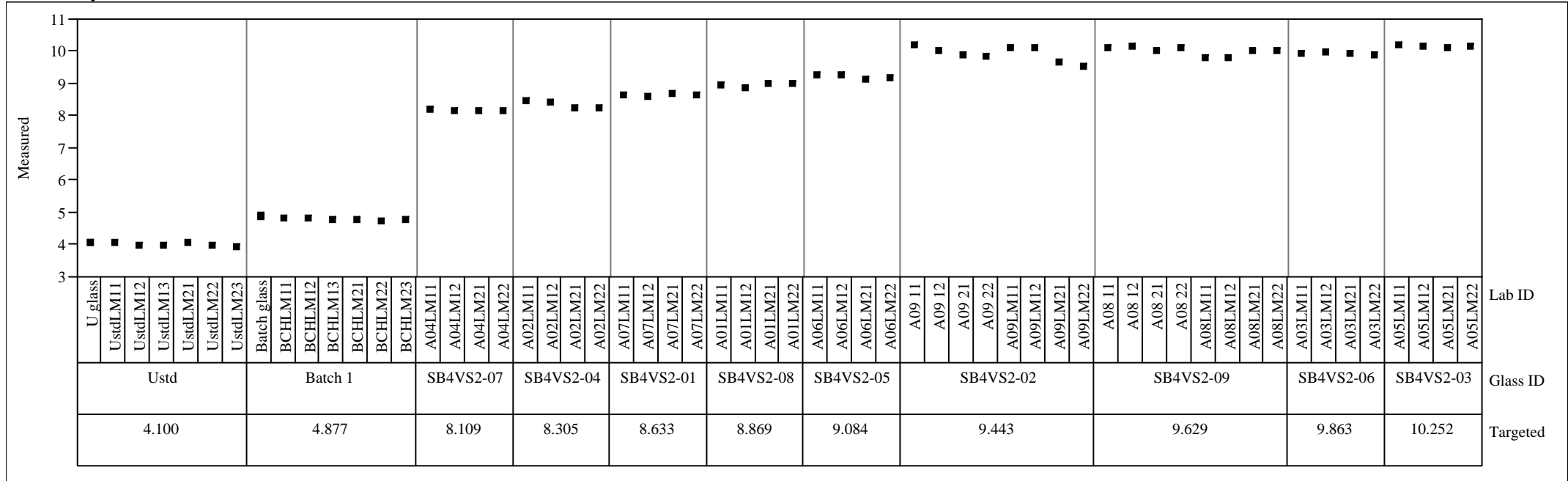

Variability Gage Oxide $=\mathrm{Al}_{2} \mathrm{O}_{3}(\mathrm{wt} \%)$

Variability Chart for Measured bc

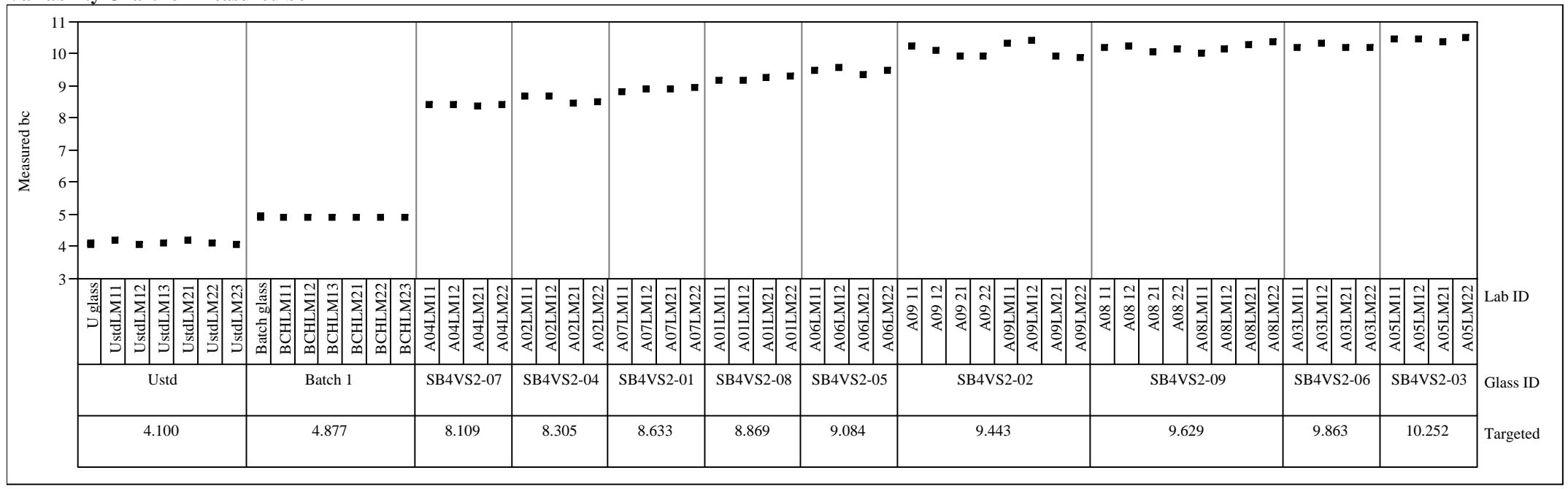


Figure A7. Oxide Measurements by Lab ID within Glass ID Including Auxiliary Measurements for LM Preps

Variability Gage Oxide=BaO (wt\%)

Variability Chart for Measured

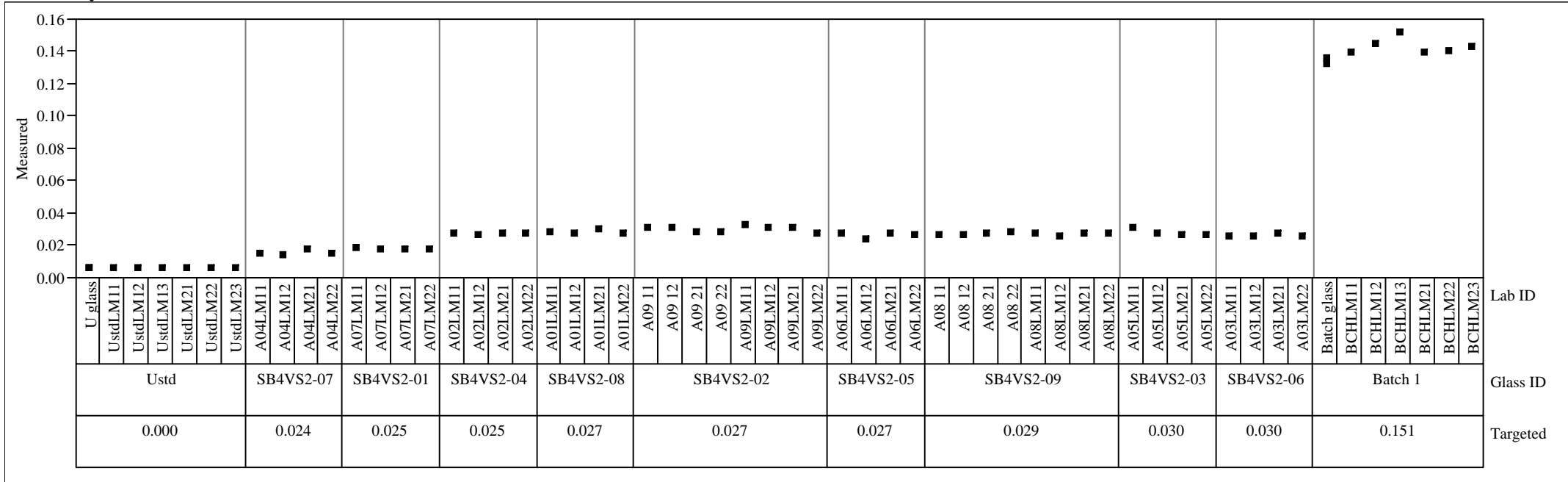

Variability Gage Oxide=BaO (wt \%)

Variability Chart for Measured bc

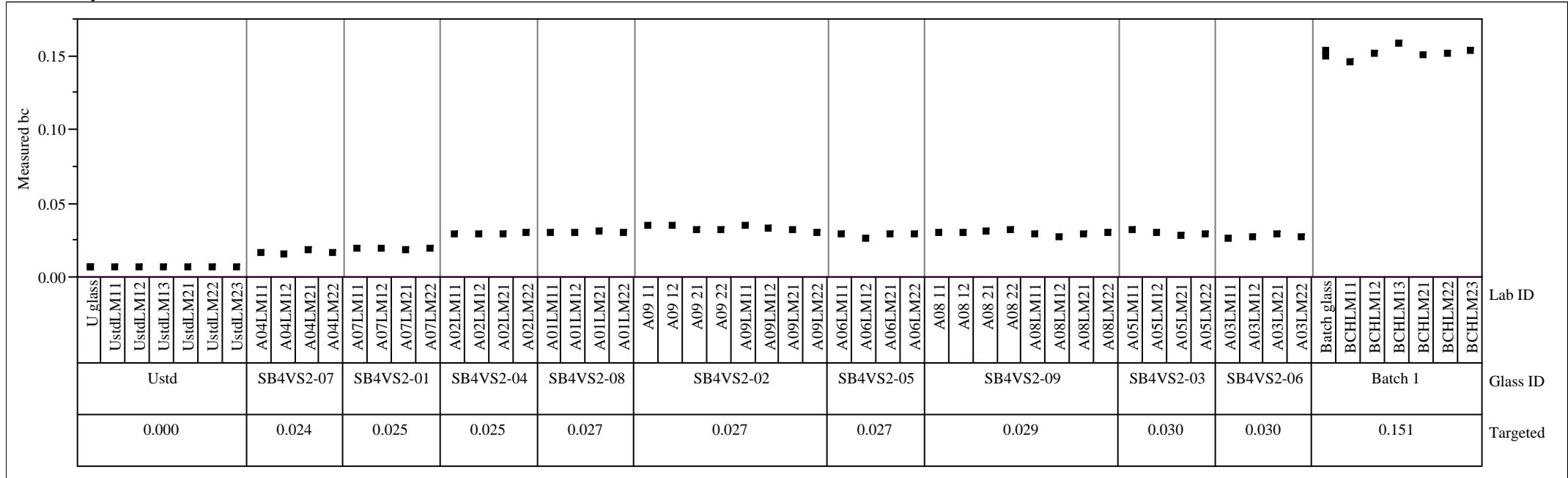




\section{Figure A7. Oxide Measurements by Lab ID within Glass ID Including Auxiliary Measurements for LM Preps}

Variability Gage Oxide $=\mathrm{CaO}($ wt $\%)$

Variability Chart for Measured

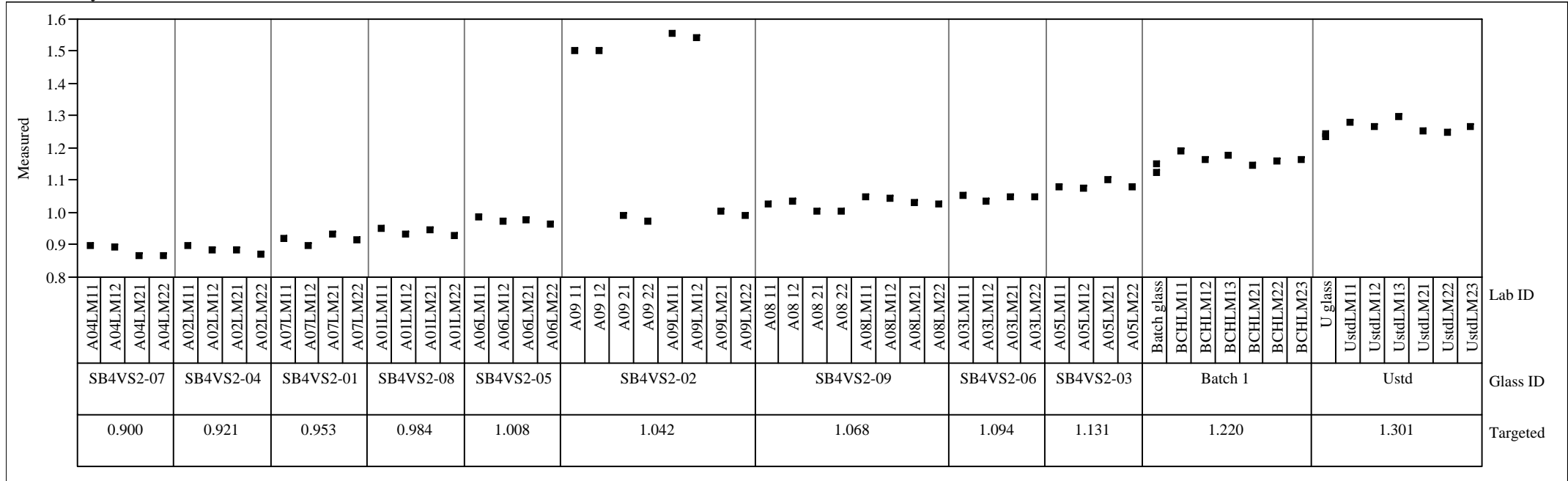

Variability Gage Oxide=CaO (wt \%)

Variability Chart for Measured bc

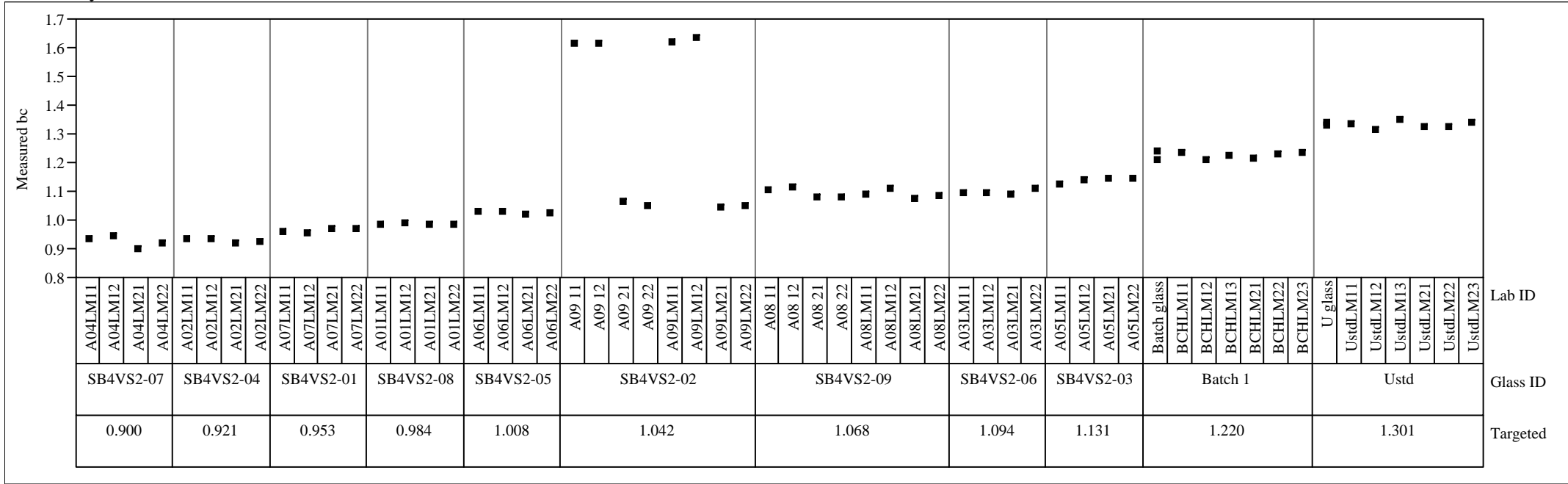


Figure A7. Oxide Measurements by Lab ID within Glass ID Including Auxiliary Measurements for LM Preps

Variability Gage Oxide $=\mathrm{Ce}_{2} \mathrm{O}_{3}(\mathrm{wt} \%)$

Variability Chart for Measured

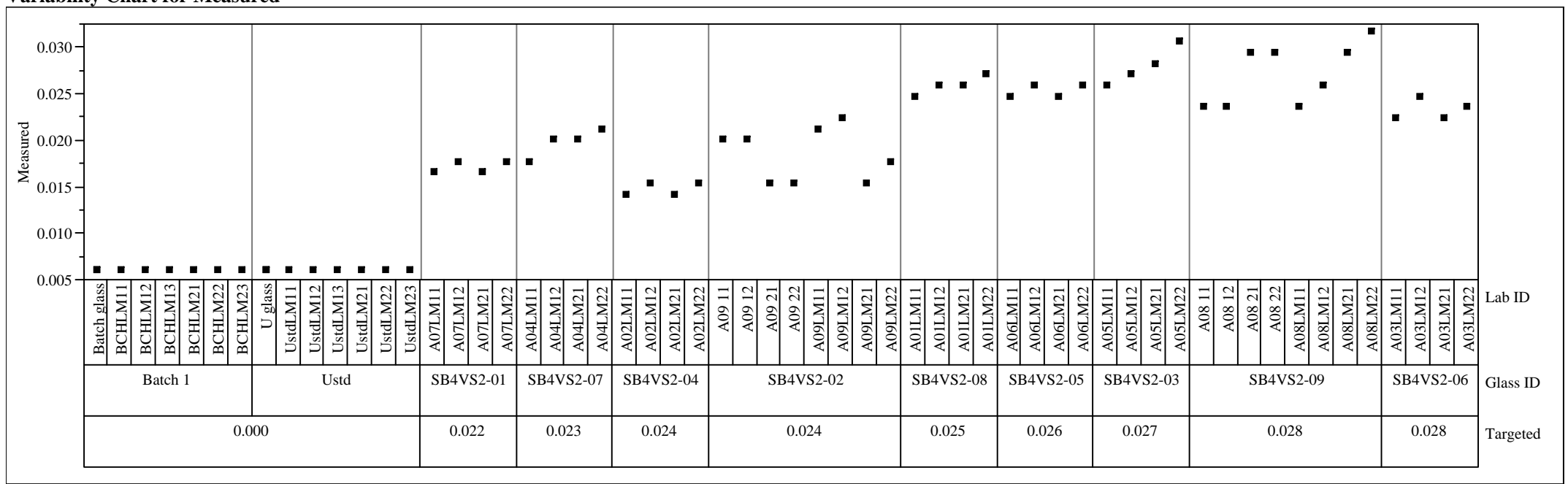

Variability Gage Oxide $=\mathrm{Ce}_{2} \mathrm{O}_{3}$ (wt\%)

Variability Chart for Measured bc

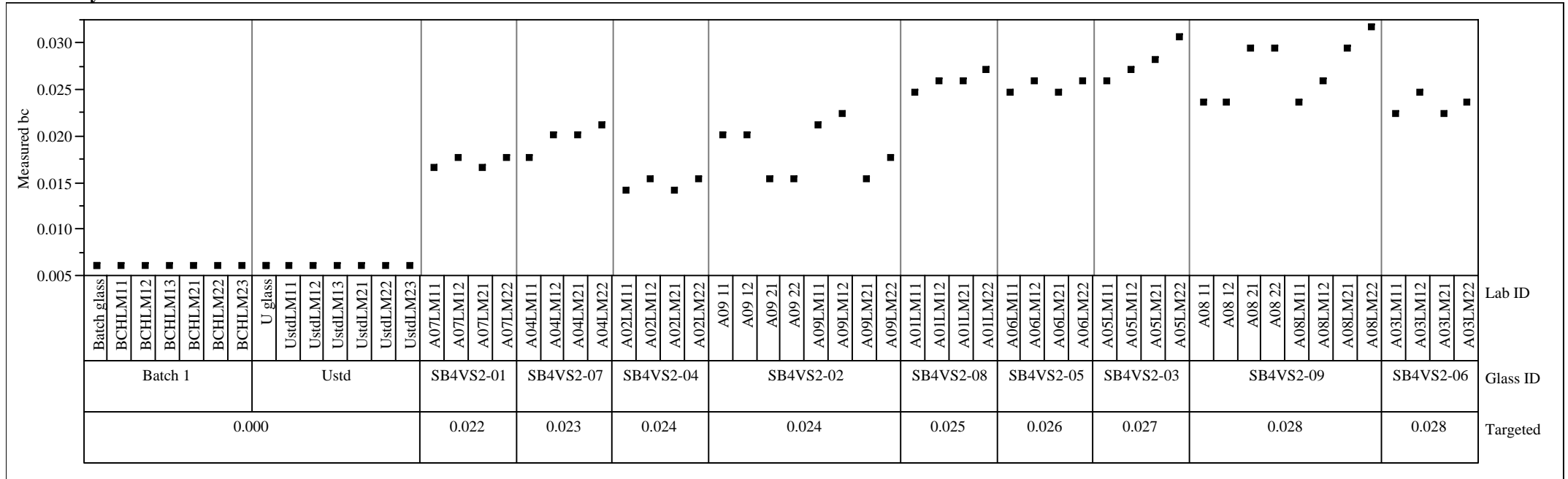


Figure A7. Oxide Measurements by Lab ID within Glass ID Including Auxiliary Measurements for LM Preps

Variability Gage Oxide $=\mathrm{Cr}_{2} \mathrm{O}_{3}(\mathrm{wt} \%)$

Variability Chart for Measured

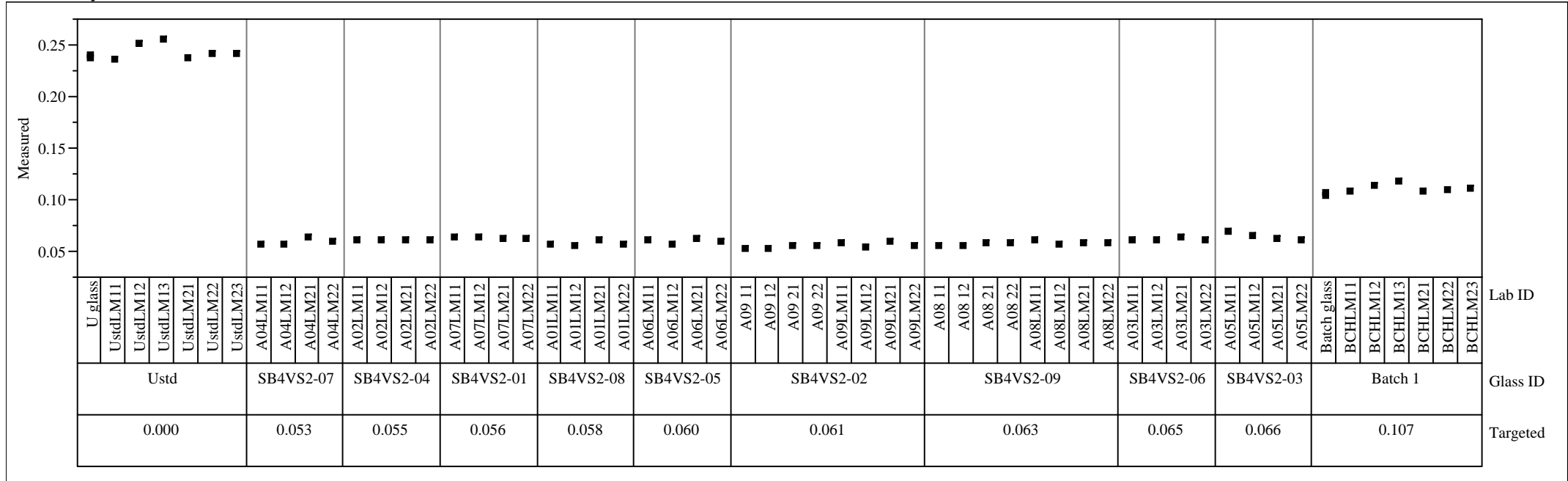

Variability Gage Oxide $=\mathrm{Cr}_{2} \mathrm{O}_{3}(\mathrm{wt} \%)$

Variability Chart for Measured bc

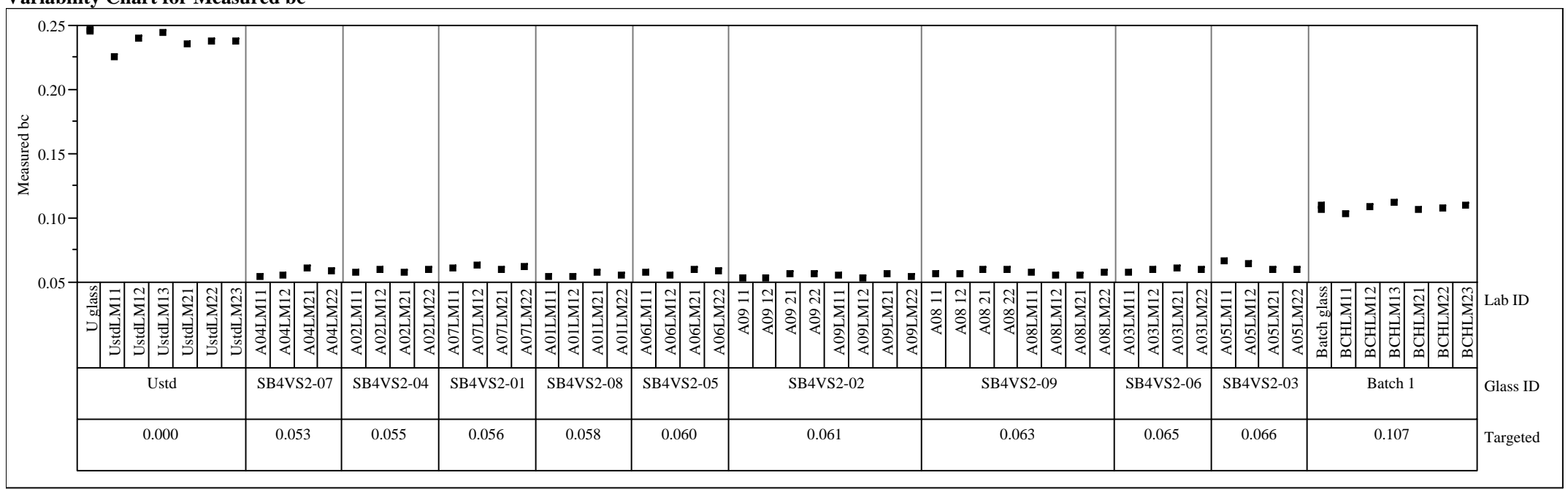


Figure A7. Oxide Measurements by Lab ID within Glass ID Including Auxiliary Measurements for LM Preps

Variability Gage Oxide $=\mathrm{CuO}($ wt $\%)$

Variability Chart for Measured

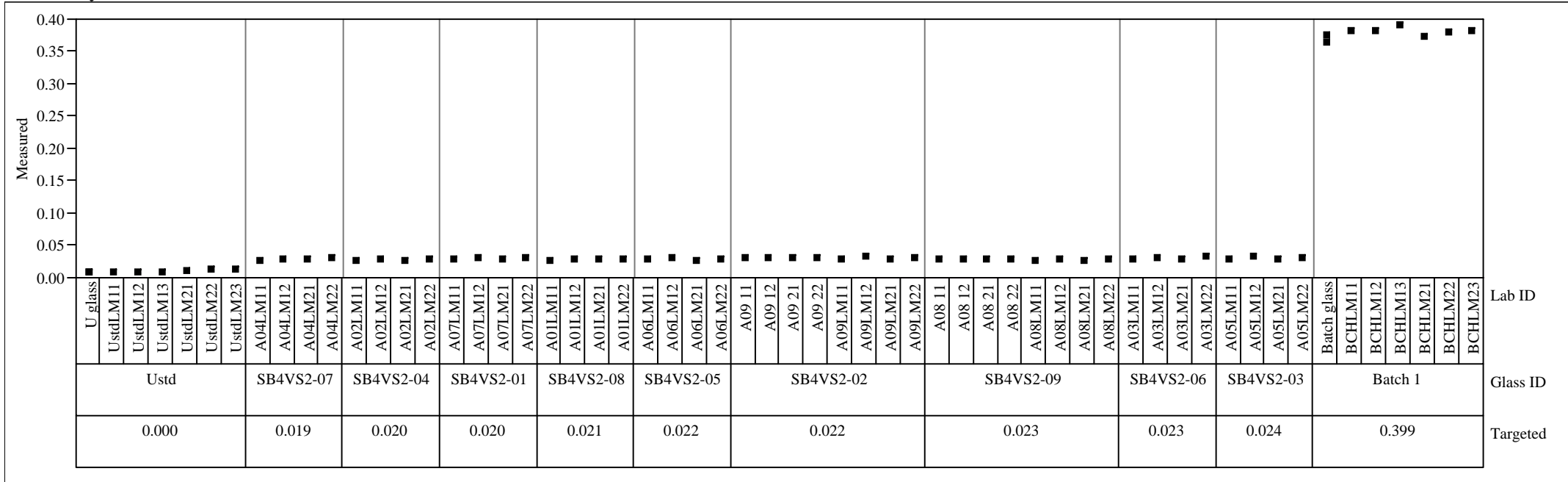

Variability Gage Oxide $=\mathrm{CuO}(\mathrm{wt} \%)$

Variability Chart for Measured bc

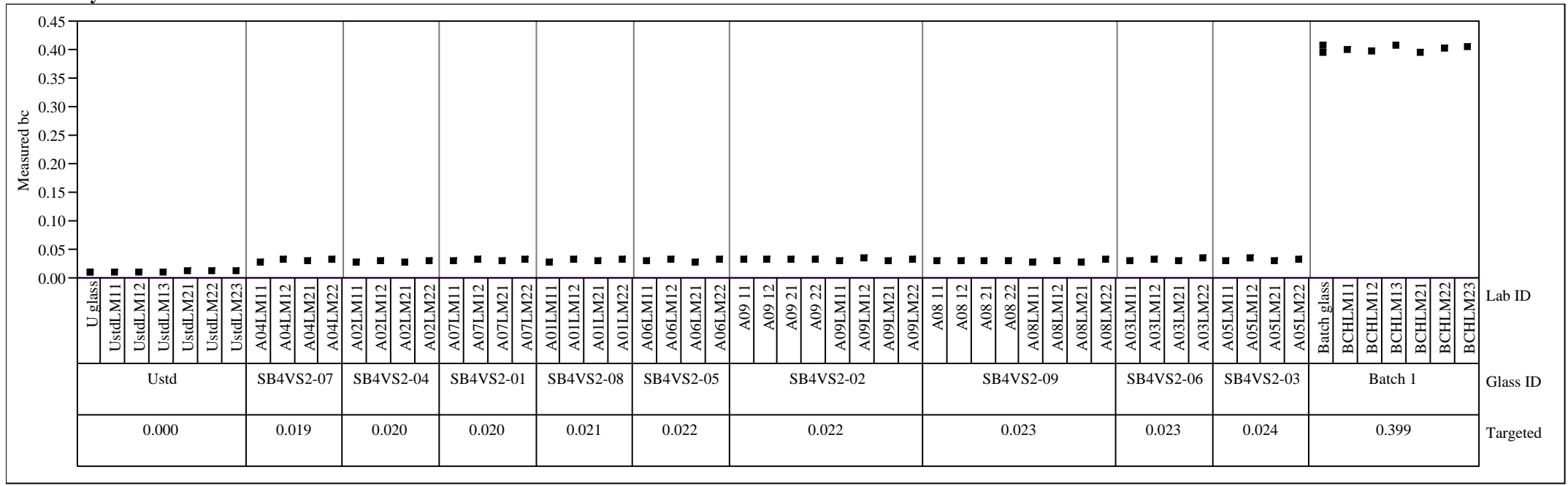




\section{Figure A7. Oxide Measurements by Lab ID within Glass ID Including Auxiliary Measurements for LM Preps}

Variability Gage Oxide $=\mathrm{La}_{2} \mathrm{O}_{3}(w t \%)$

Variability Chart for Measured

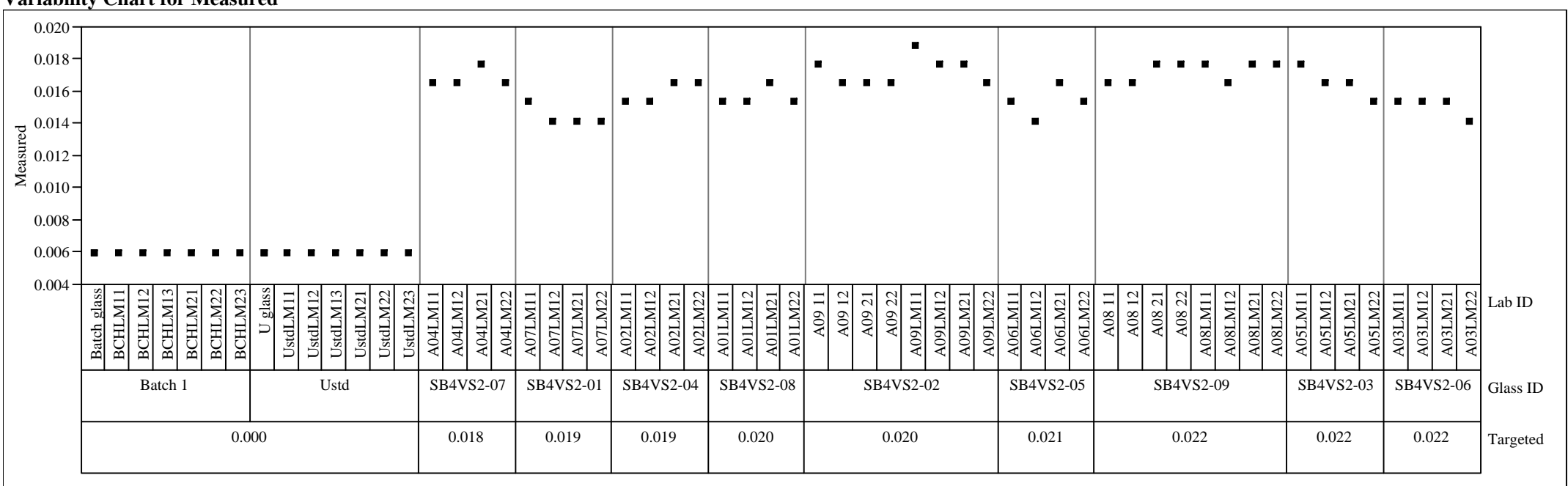

Variability Gage Oxide $=\mathrm{La}_{2} \mathrm{O}_{3}(\mathrm{wt} \%)$

Variability Chart for Measured bc

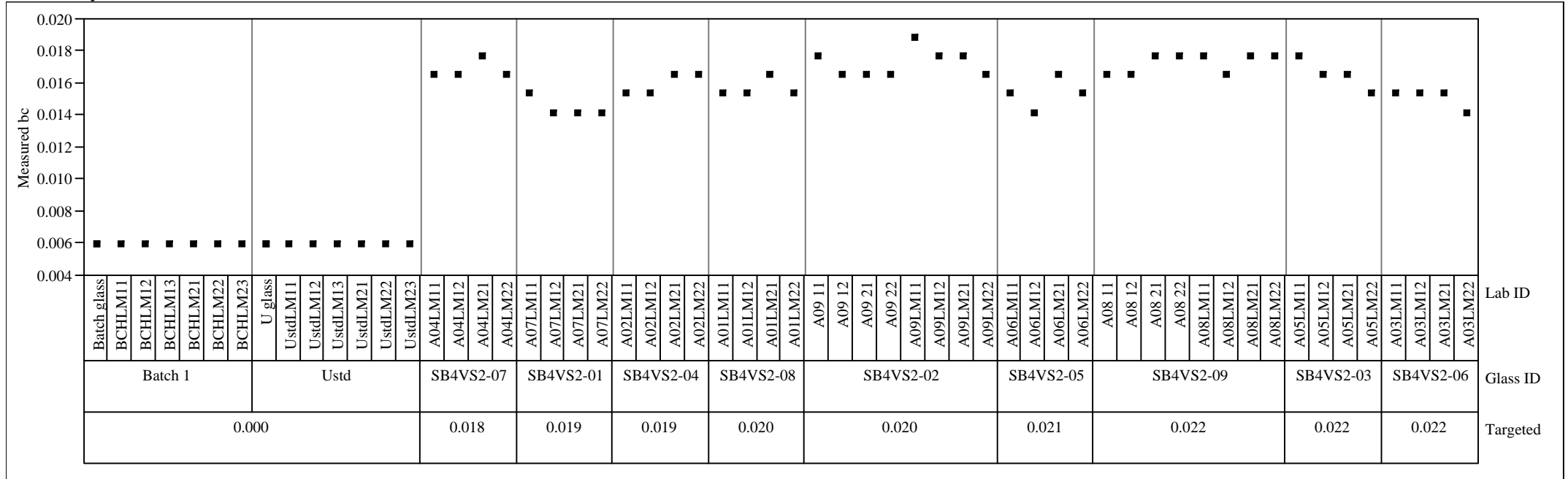




\section{Figure A7. Oxide Measurements by Lab ID within Glass ID Including Auxiliary Measurements for LM Preps}

Variability Gage Oxide=MgO (wt\%)

Variability Chart for Measured

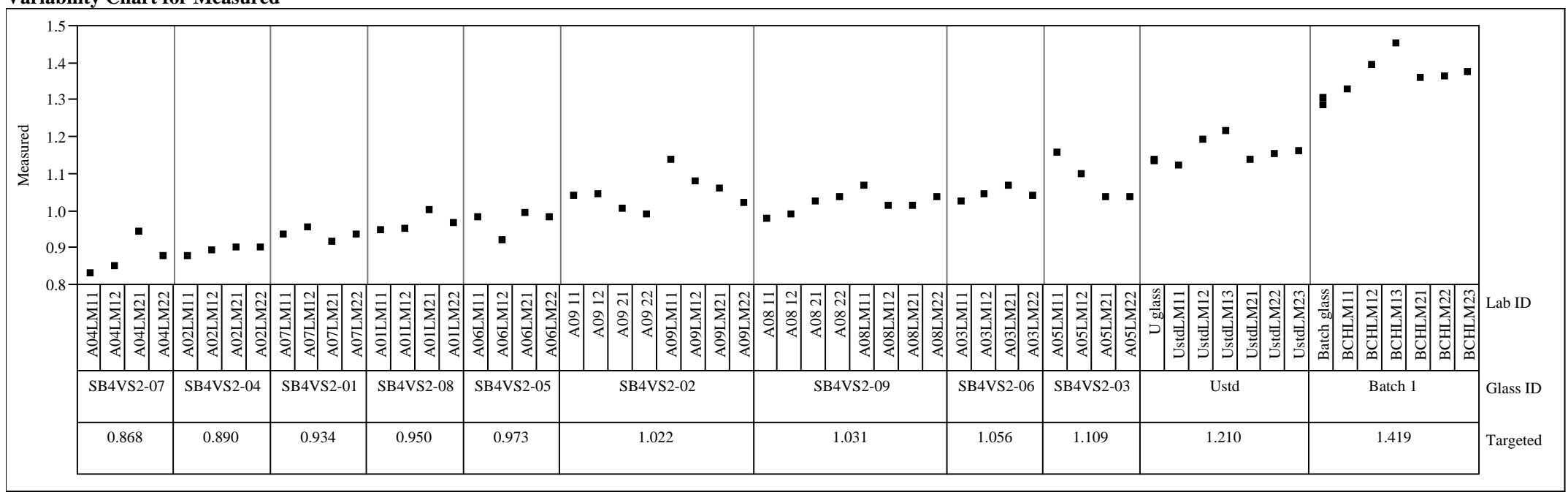

Variability Gage Oxide=MgO (wt \%)

Variability Chart for Measured bc

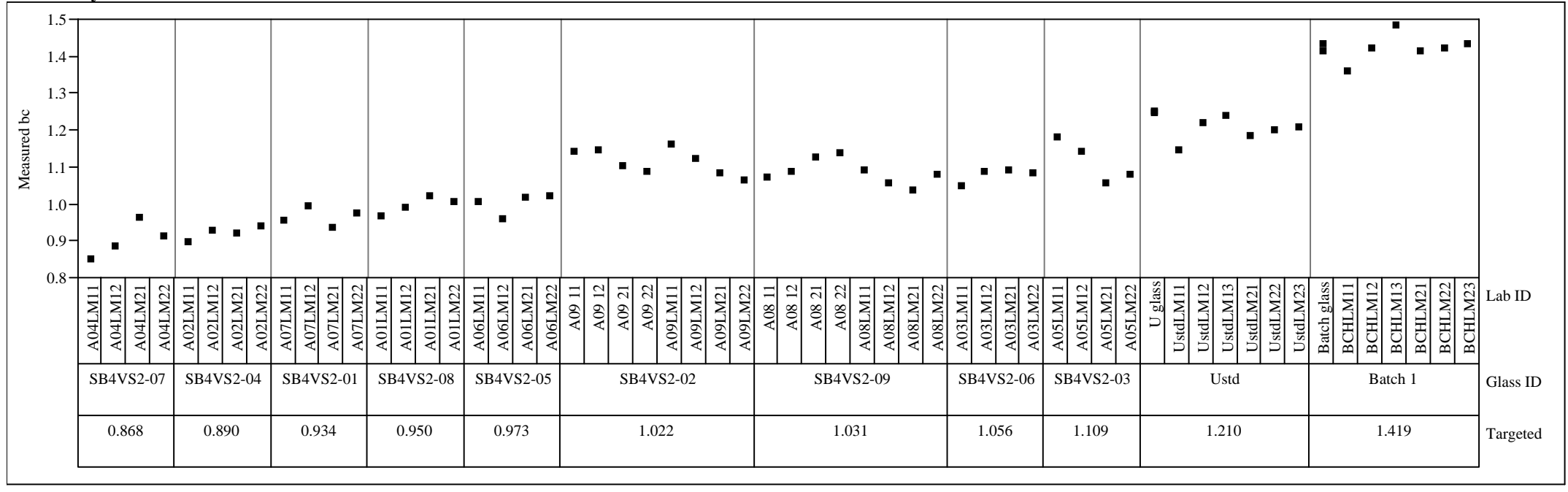




\section{Figure A7. Oxide Measurements by Lab ID within Glass ID Including Auxiliary Measurements for LM Preps}

Variability Gage Oxide=MnO (wt\%)

Variability Chart for Measured

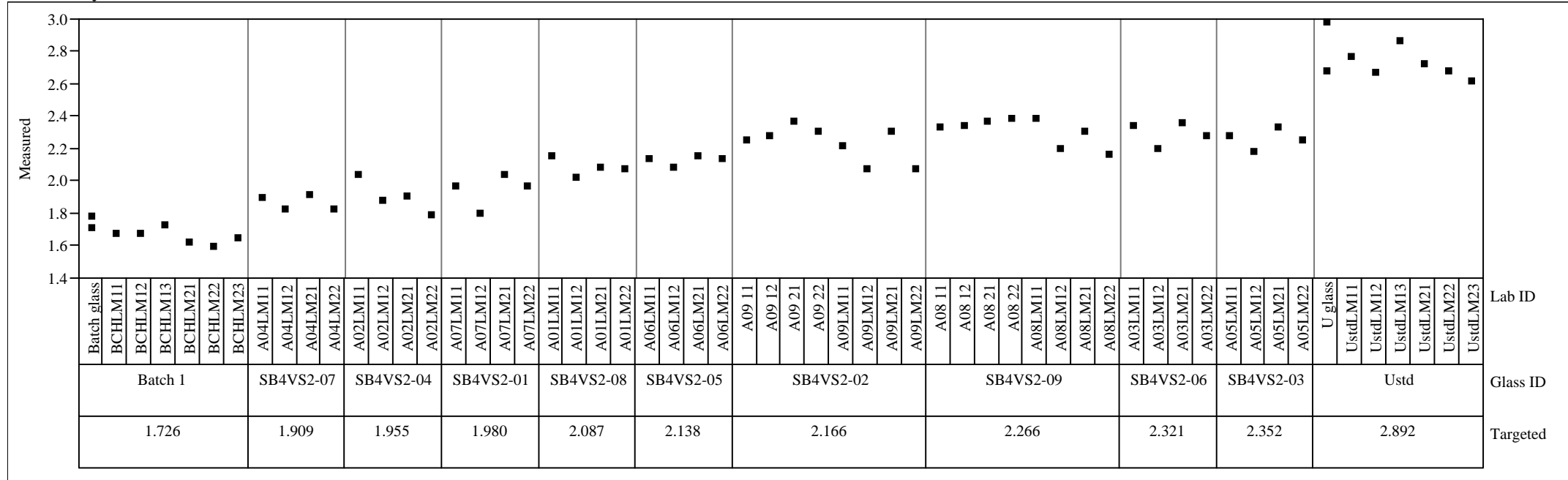

Variability Gage Oxide= $\mathrm{MnO}$ (wt \%)

Variability Chart for Measured bc

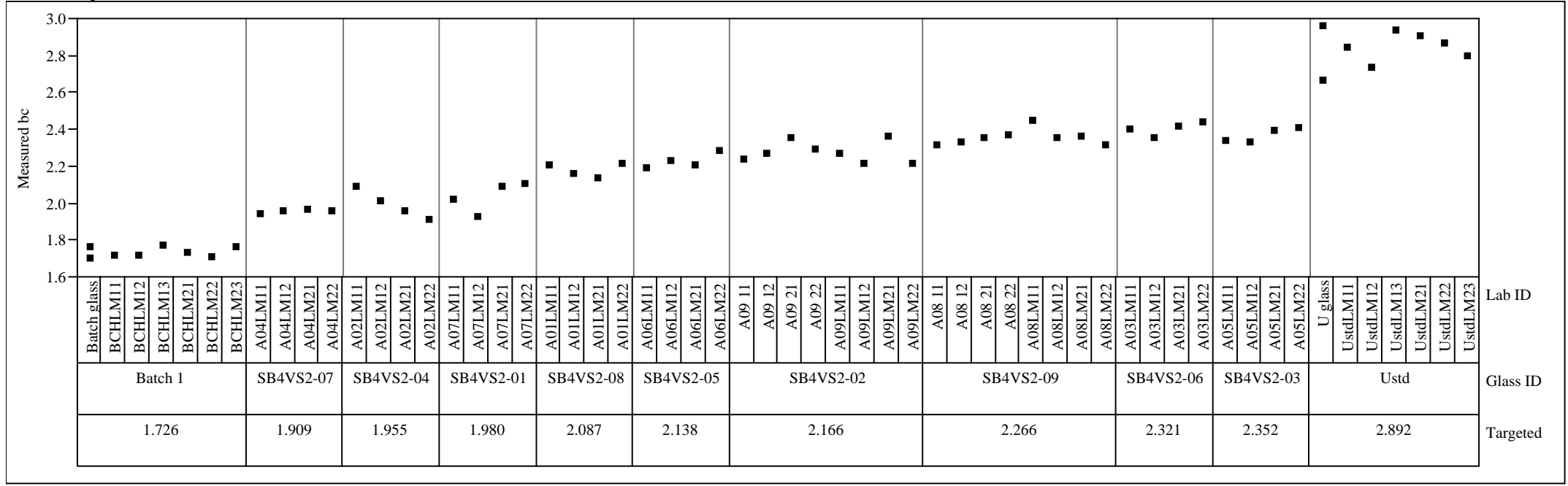


Figure A7. Oxide Measurements by Lab ID within Glass ID Including Auxiliary Measurements for LM Preps

Variability Gage Oxide $=\mathrm{Na}_{2} \mathrm{O}(\mathrm{wt} \%)$

Variability Chart for Measured

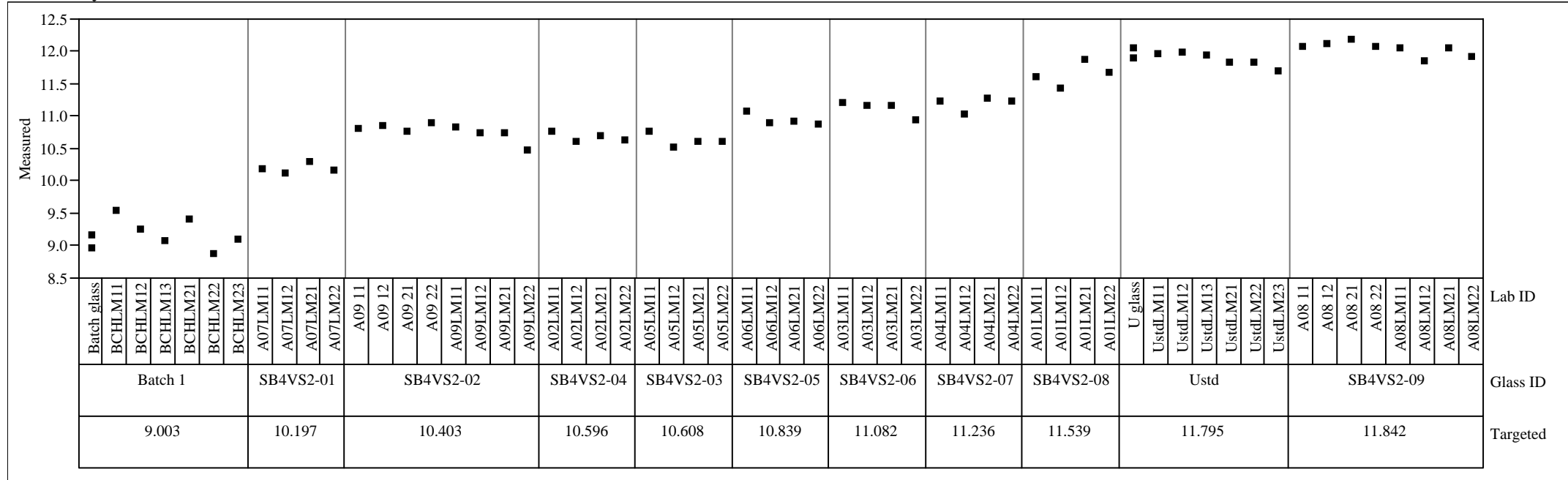

Variability Gage Oxide $=\mathrm{Na}_{2} \mathrm{O}(\mathrm{wt} \%)$

Variability Chart for Measured bc

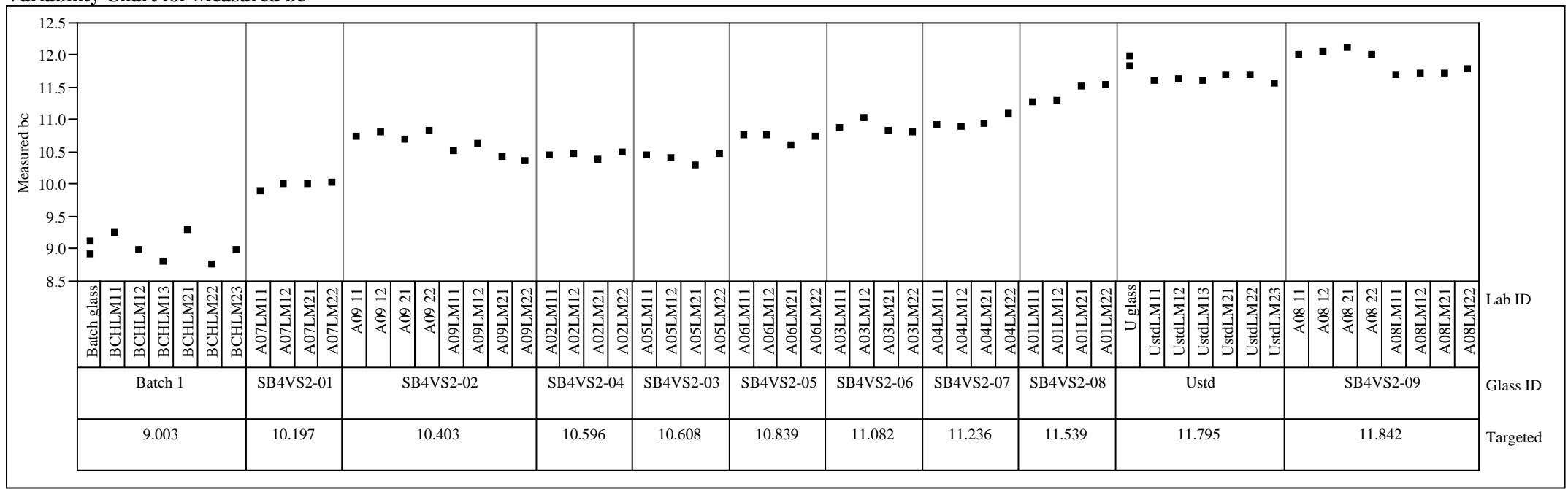




\section{Figure A7. Oxide Measurements by Lab ID within Glass ID Including Auxiliary Measurements for LM Preps}

Variability Gage Oxide=NiO (wt \%)

Variability Chart for Measured

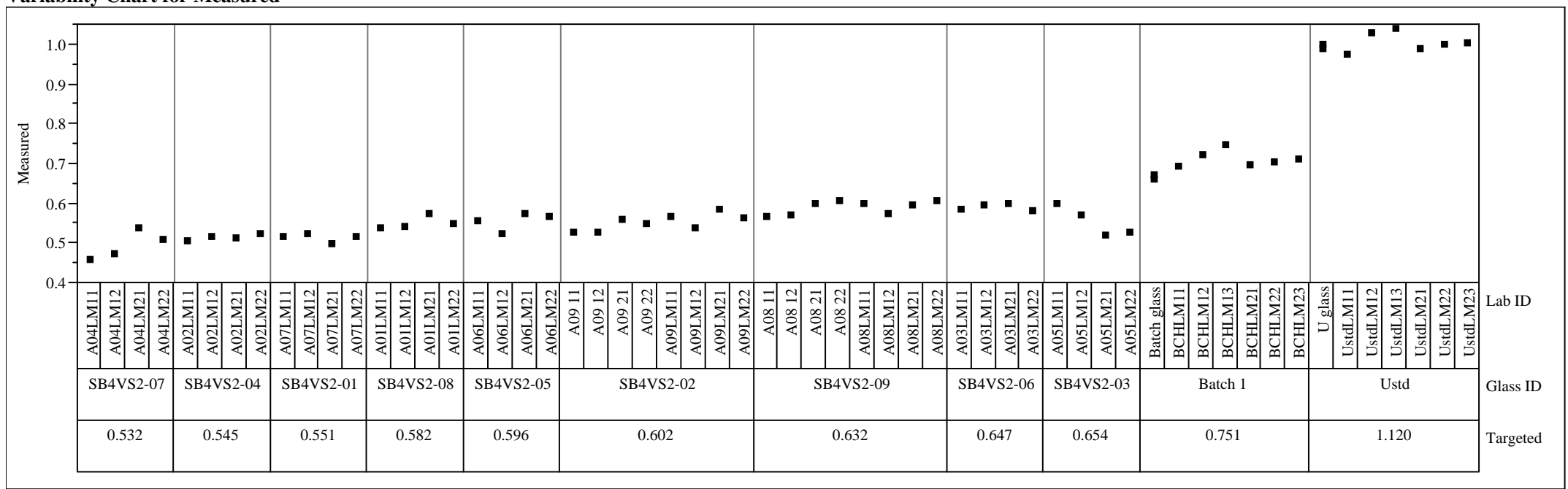

Variability Gage Oxide=NiO (wt\%)

Variability Chart for Measured bc

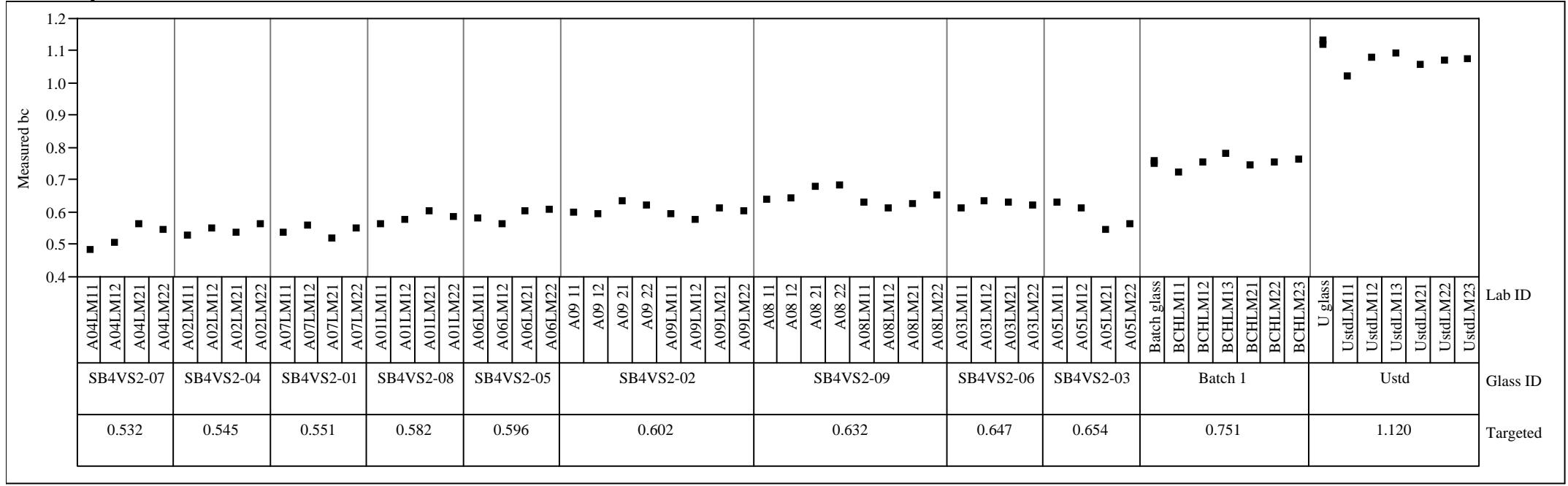




\section{Figure A7. Oxide Measurements by Lab ID within Glass ID Including Auxiliary Measurements for LM Preps}

Variability Gage Oxide=PbO (wt\%)

Variability Chart for Measured

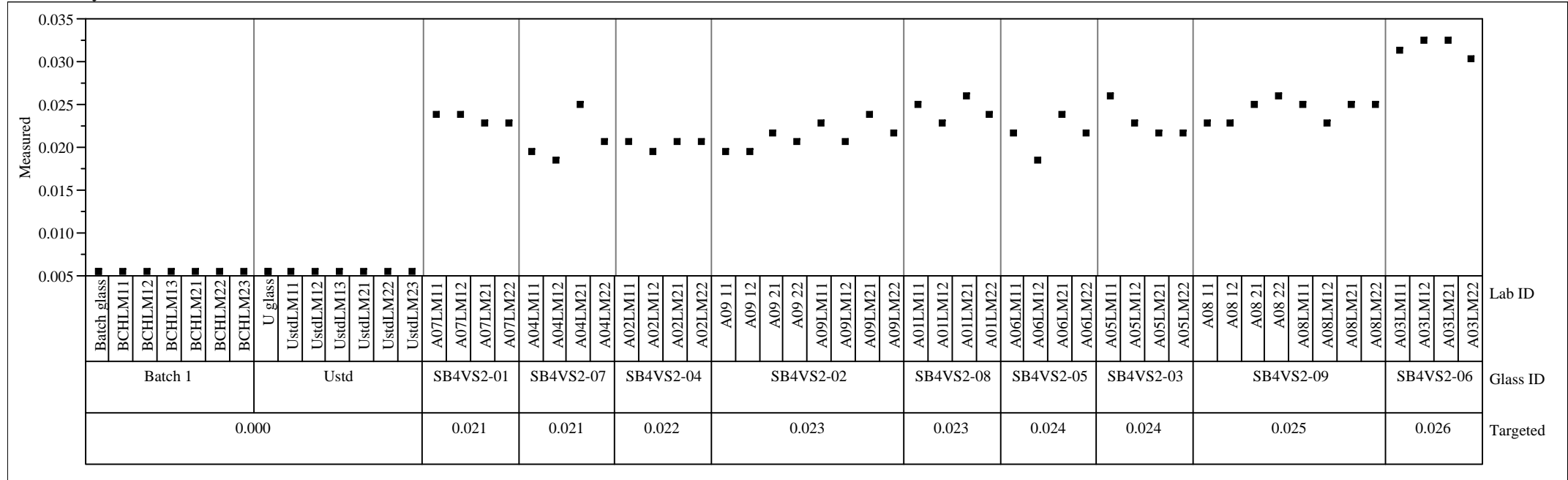

Variability Gage Oxide=PbO (wt\%)

Variability Chart for Measured bc

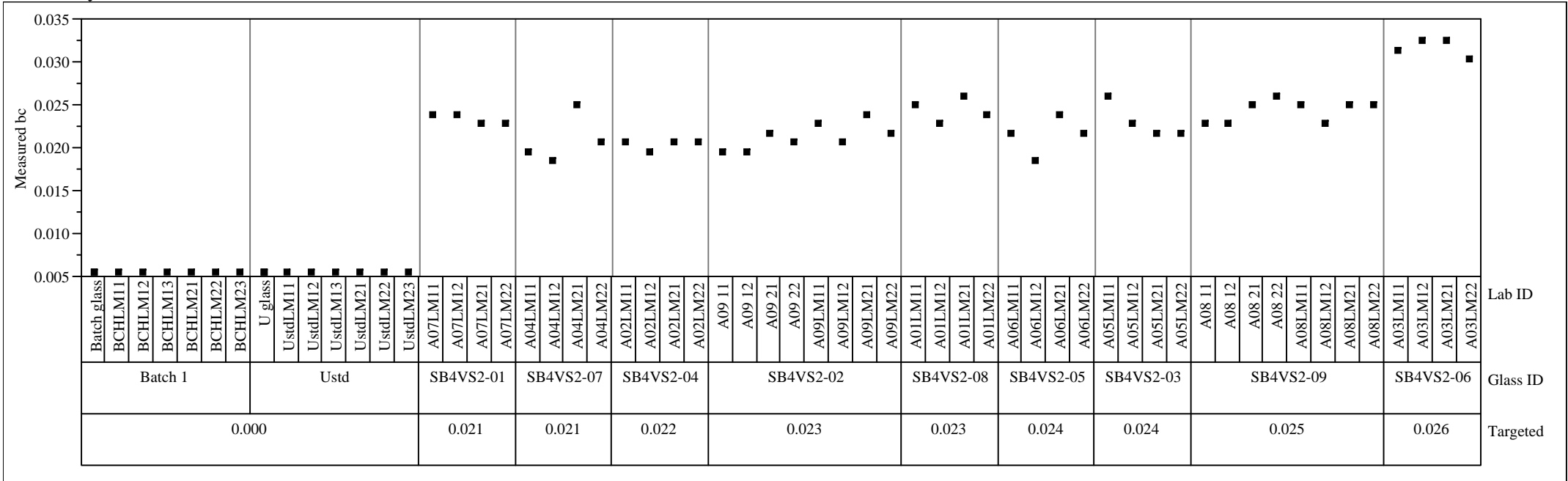


Figure A7. Oxide Measurements by Lab ID within Glass ID Including Auxiliary Measurements for LM Preps

Variability Gage Oxide $=\mathrm{SO}_{4}(\mathrm{wt} \%)$

Variability Chart for Measured

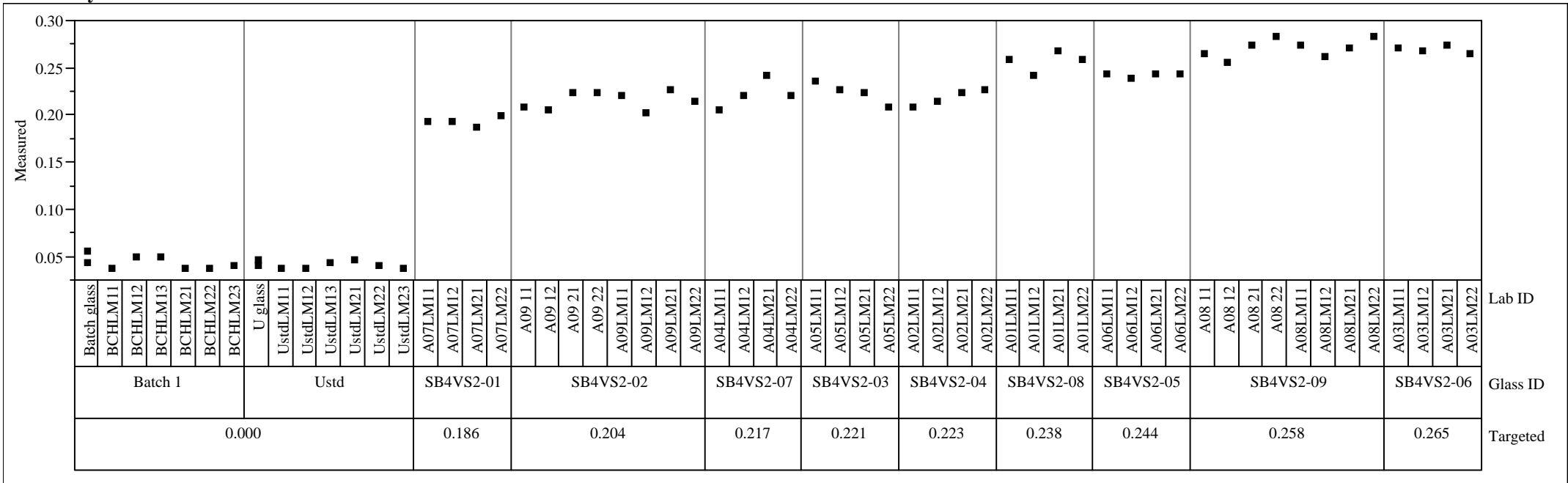

Variability Gage Oxide $=\mathrm{SO}_{4}(\mathrm{wt} \%)$

Variability Chart for Measured bc

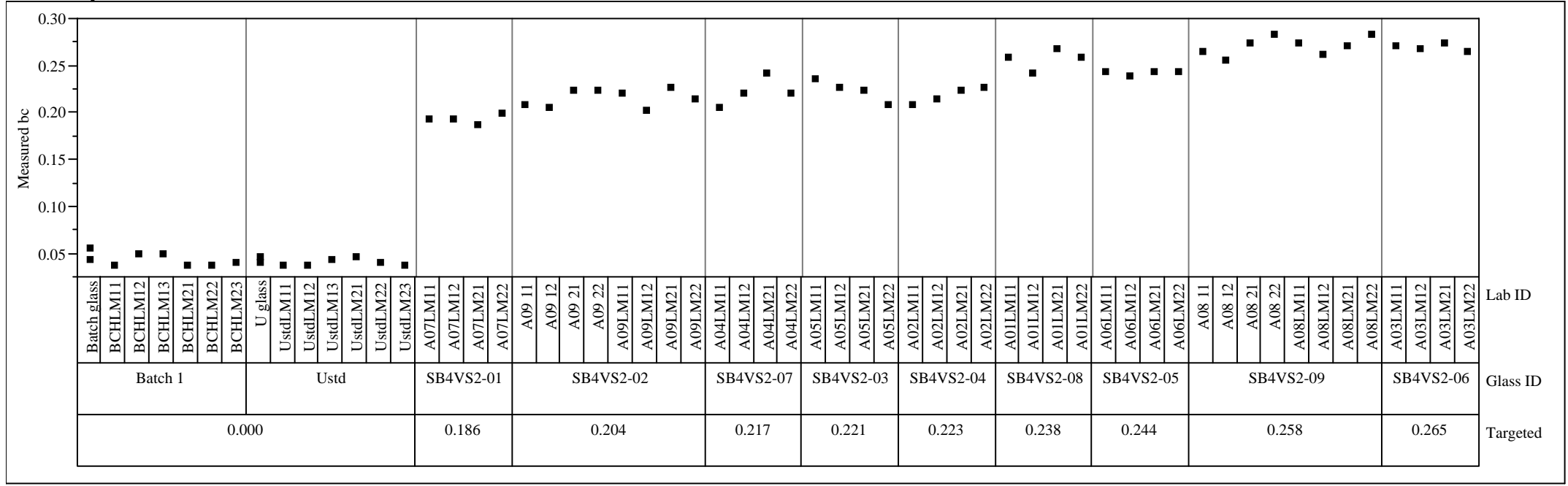




\section{Figure A7. Oxide Measurements by Lab ID within Glass ID Including Auxiliary Measurements for LM Preps}

Variability Gage Oxide $=\mathrm{SiO}_{2}(\mathrm{wt} \%)$

Variability Chart for Measured

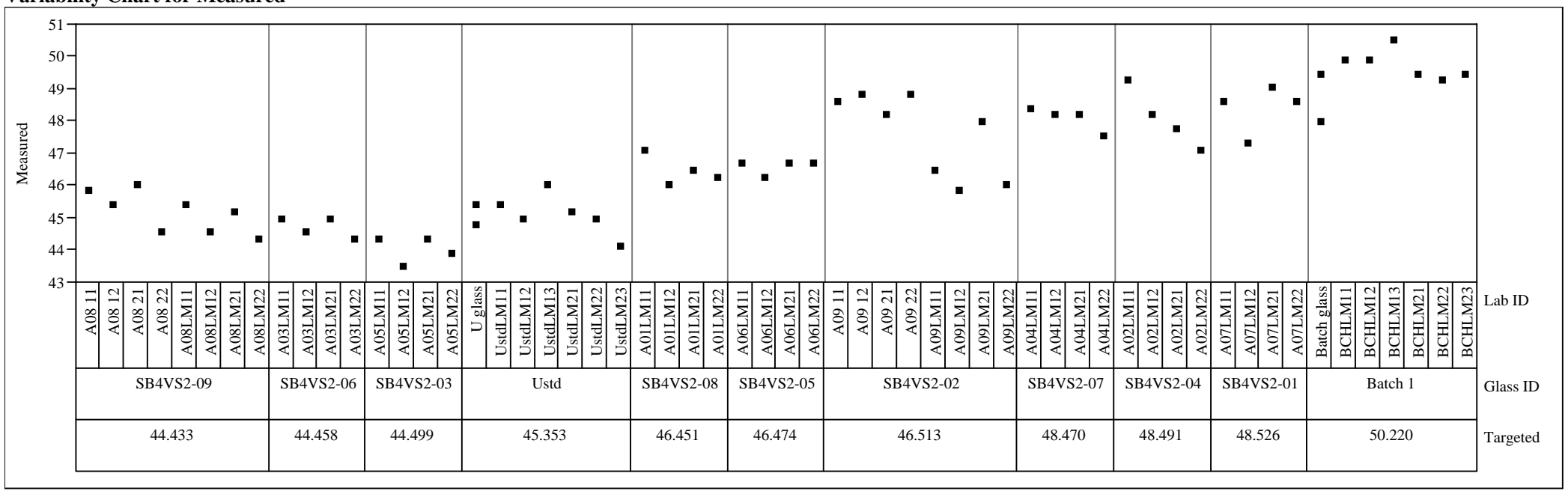

Variability Gage Oxide $=\mathrm{SiO}_{2}(\mathrm{wt} \%)$

Variability Chart for Measured bc

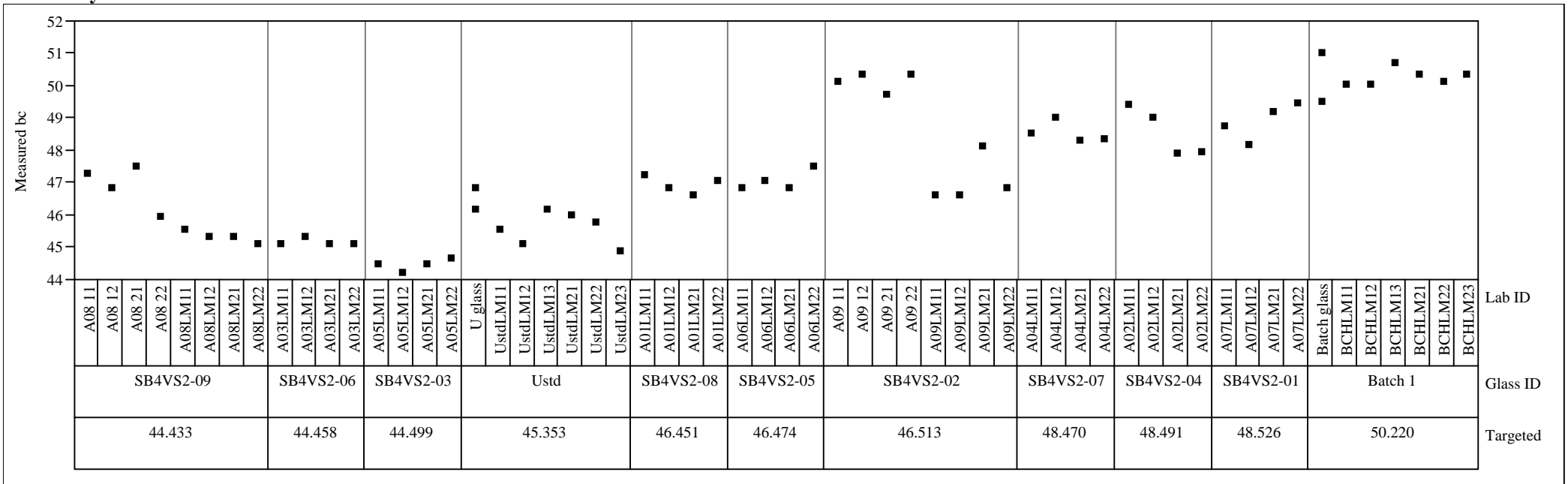




\section{Figure A7. Oxide Measurements by Lab ID within Glass ID Including Auxiliary Measurements for LM Preps}

Variability Gage Oxide $=\mathrm{TiO}_{2}(\mathrm{wt} \%)$

Variability Chart for Measured

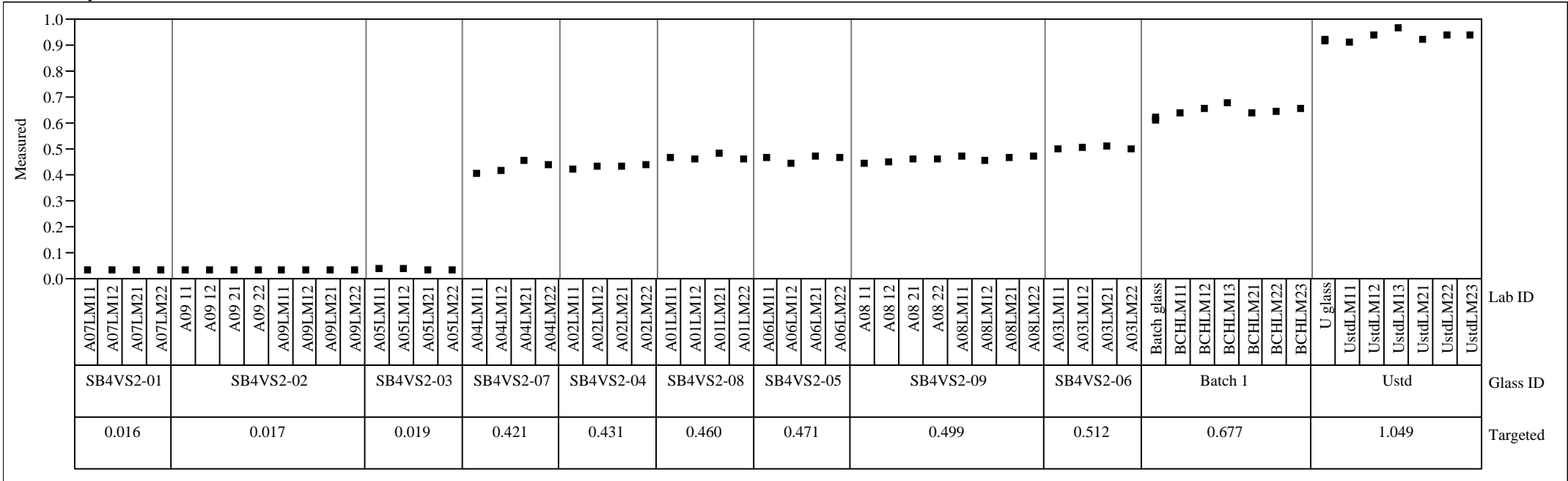

Variability Gage Oxide $=\mathrm{TiO}_{2}(\mathrm{wt} \%)$

Variability Chart for Measured bc

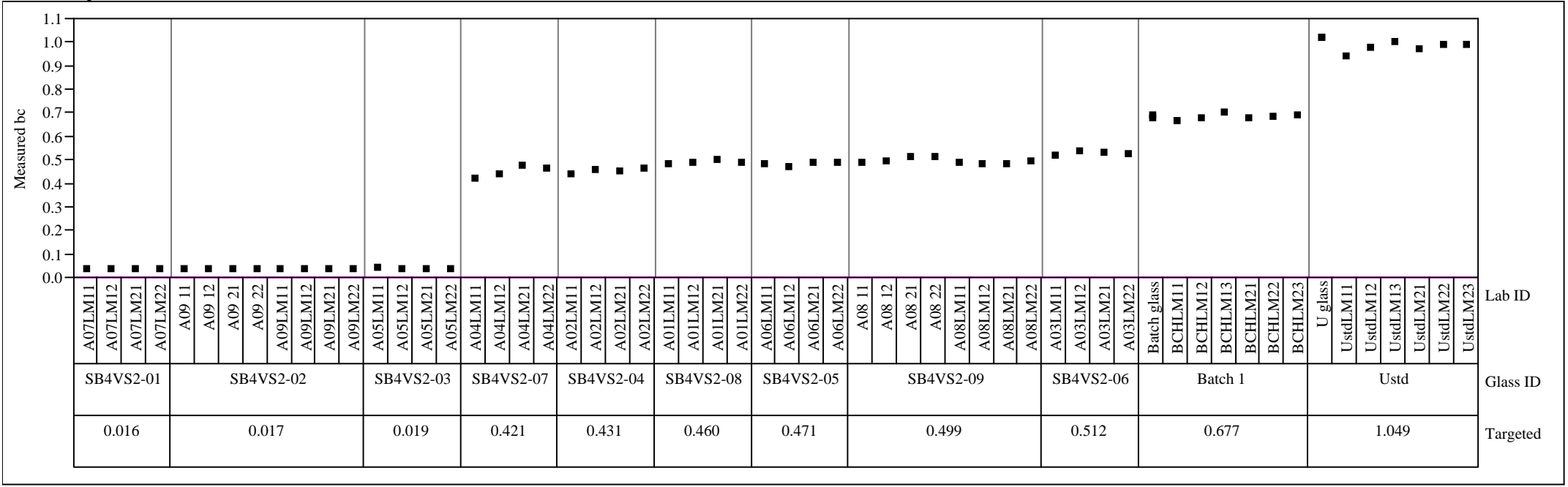


Figure A7. Oxide Measurements by Lab ID within Glass ID Including Auxiliary Measurements for LM Preps

Variability Gage Oxide $=\mathrm{U}_{3} \mathrm{O}_{8}(\mathrm{wt} \%)$

Variability Chart for Measured

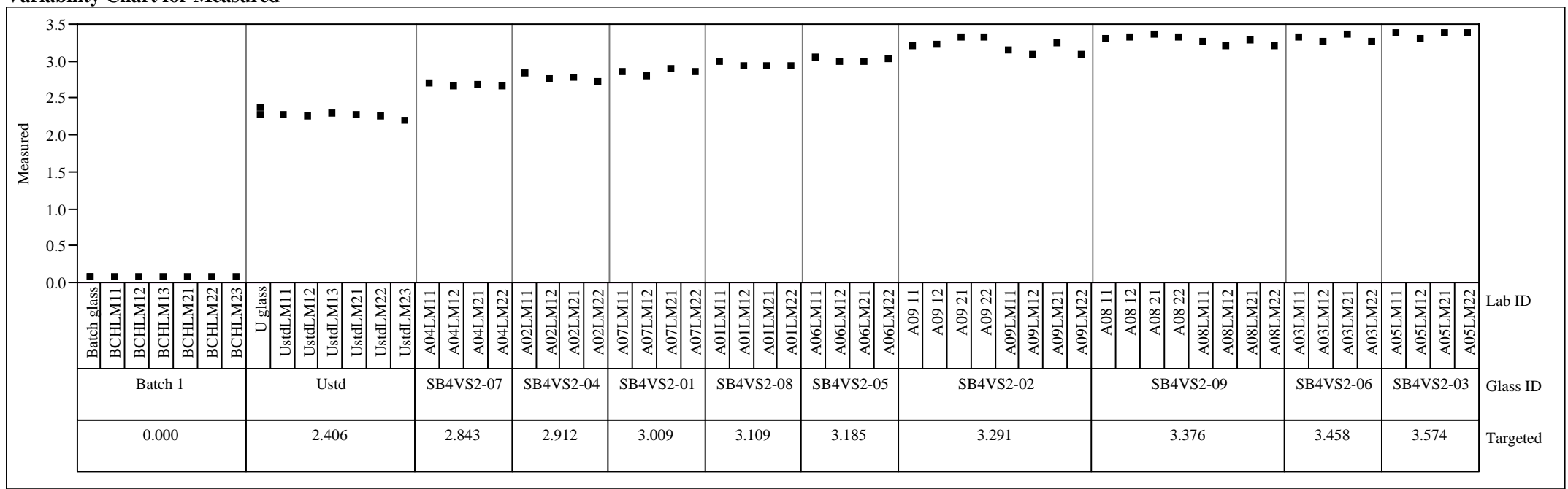

Variability Gage Oxide $=\mathrm{U}_{3} \mathrm{O}_{8}(\mathrm{wt} \%)$

Variability Chart for Measured bc

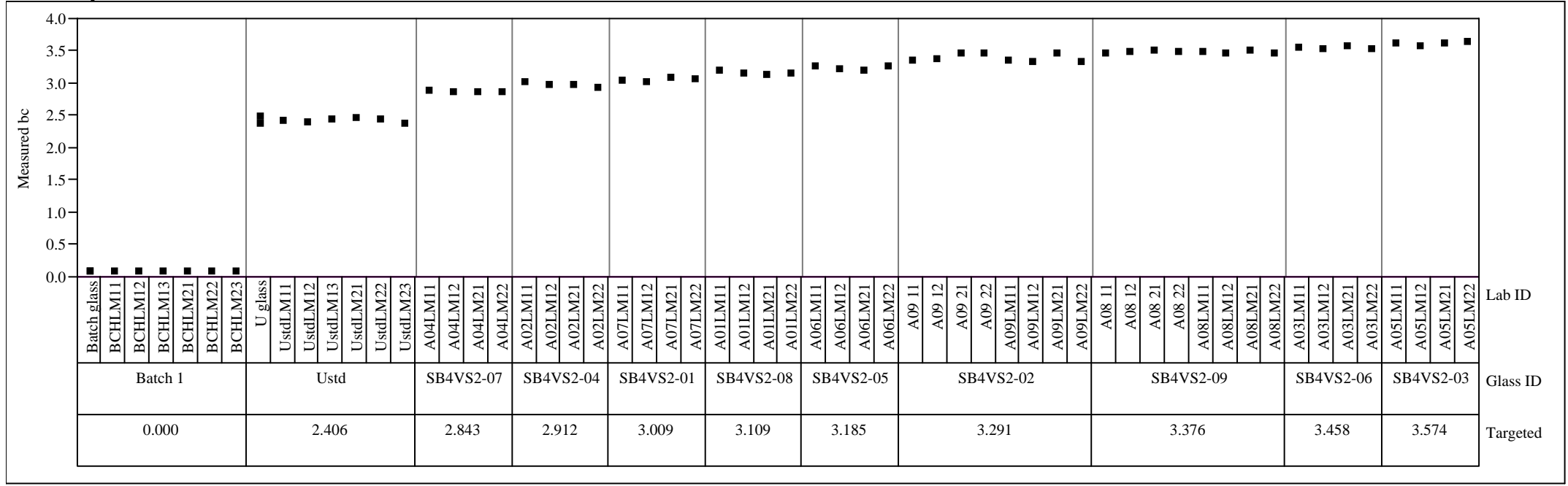


Figure A7. Oxide Measurements by Lab ID within Glass ID Including Auxiliary Measurements for LM Preps

Variability Gage Oxide $=\mathrm{ZrO}_{2}(\mathrm{wt} \%)$

Variability Chart for Measured

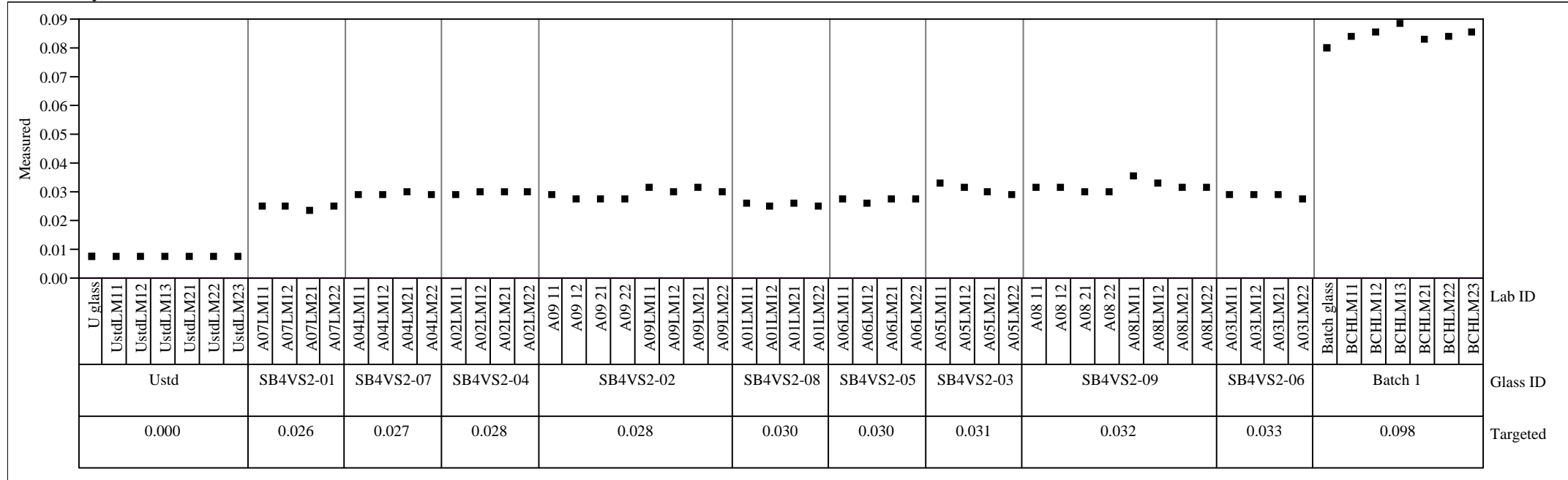

Variability Gage Oxide $=\mathrm{ZrO}_{2}(\mathrm{wt} \%)$

Variability Chart for Measured bc

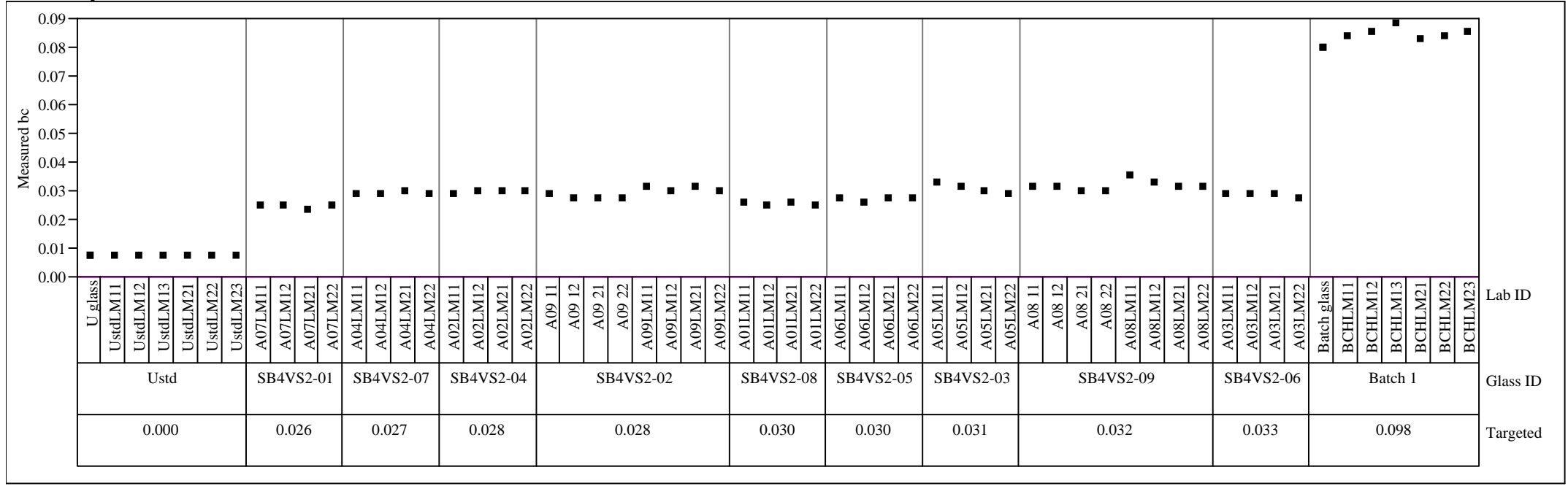


Figure A8. Oxide Measurements by Lab ID within Glass ID Including Auxiliary Measurements for LM Preps

Variability Gage Oxide $=\mathrm{B}_{2} \mathrm{O}_{3}(\mathrm{wt} \%)$

Variability Chart for Measured

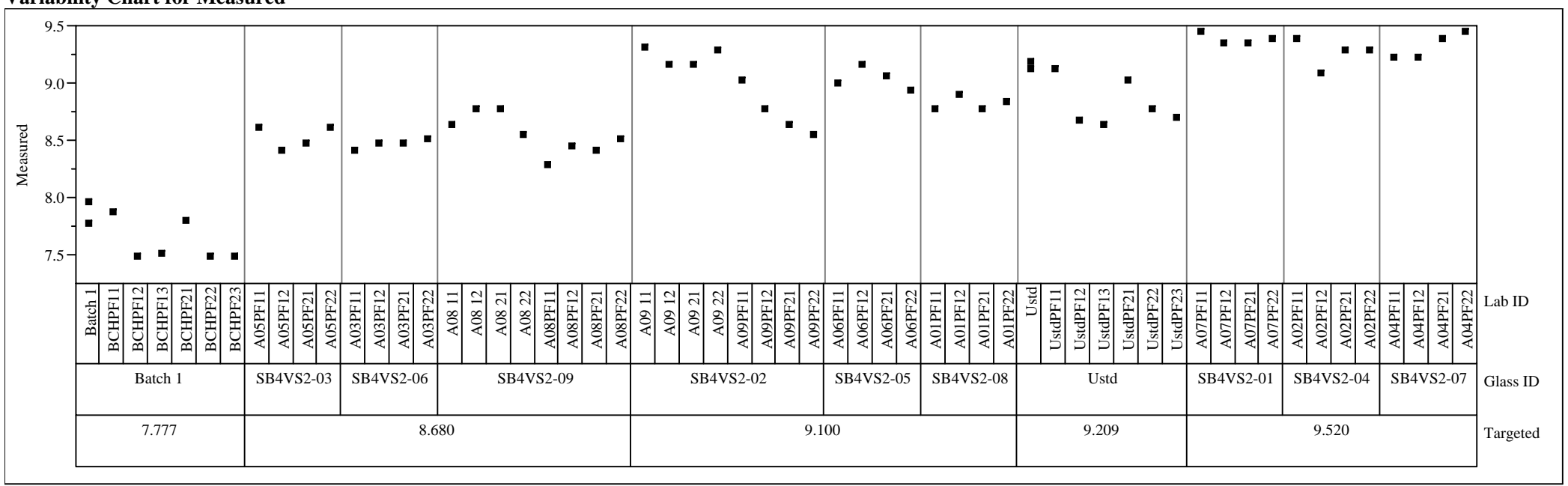

Variability Gage Oxide $=\mathrm{B}_{2} \mathrm{O}_{3}(\mathrm{wt} \%)$

Variability Chart for Measured bc

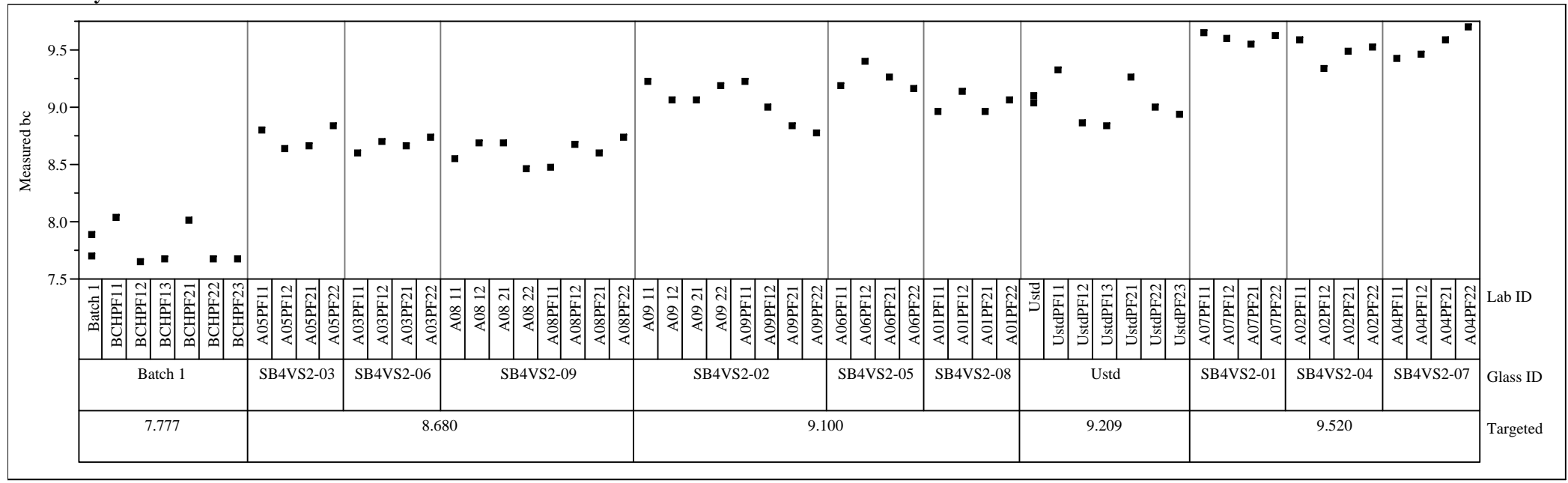




\section{Figure A8. Oxide Measurements by Lab ID within Glass ID Including Auxiliary Measurements for LM Preps}

Variability Gage Oxide $=\mathrm{Fe}_{2} \mathrm{O}_{3}(\mathrm{wt} \%)$

Variability Chart for Measured

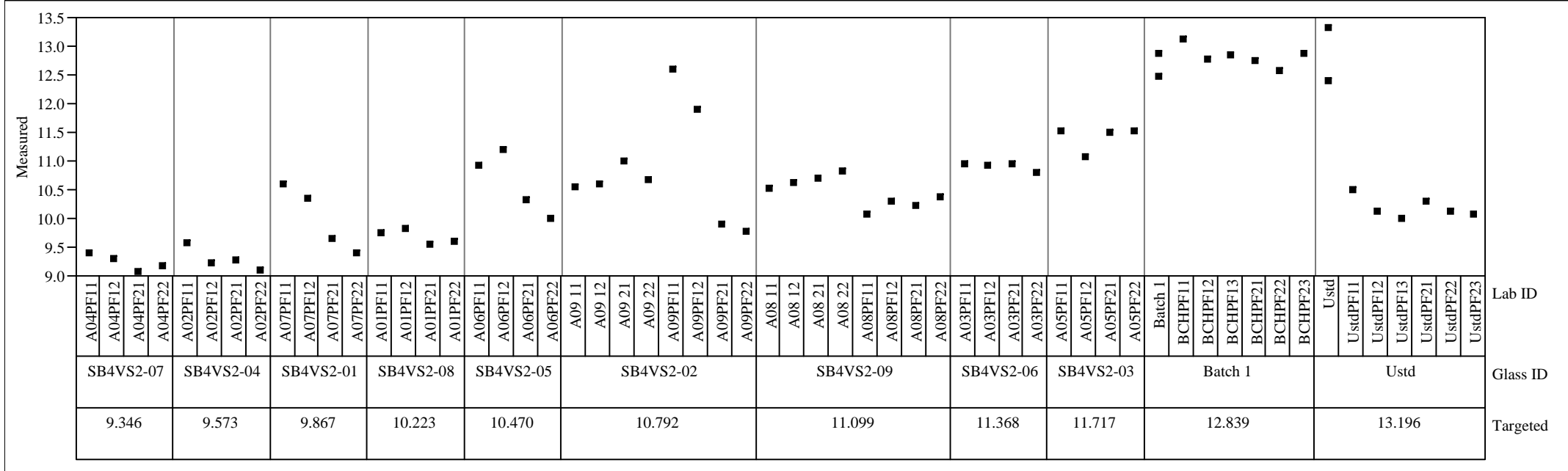

Variability Gage Oxide $=\mathrm{Fe}_{2} \mathrm{O}_{3}(\mathrm{wt} \%)$

Variability Chart for Measured bc

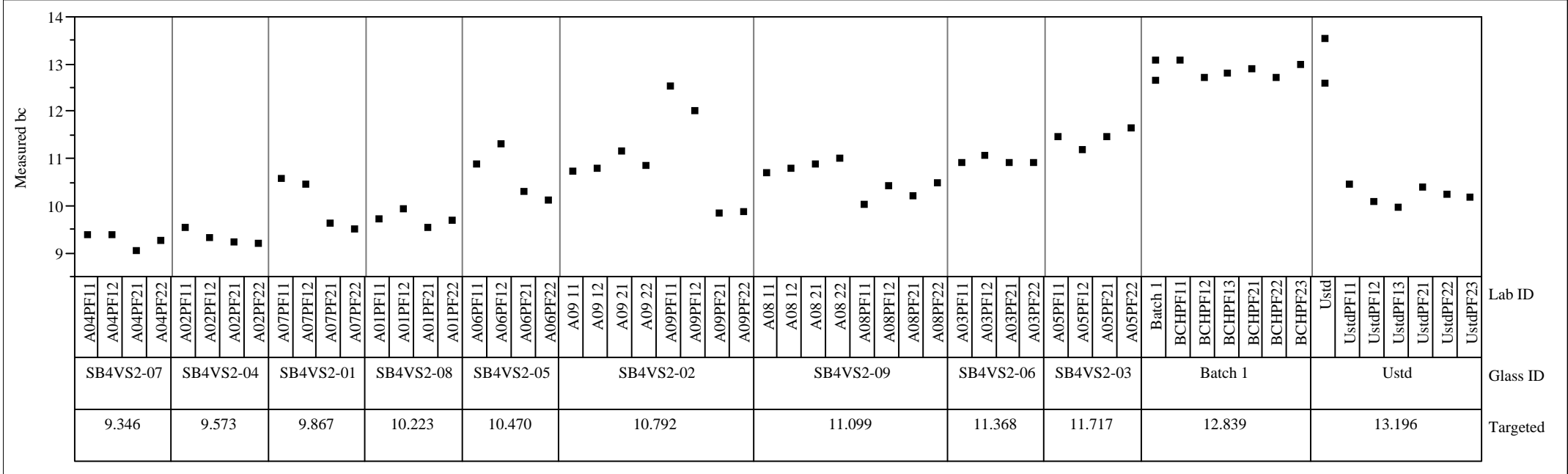


Figure A8. Oxide Measurements by Lab ID within Glass ID Including Auxiliary Measurements for LM Preps

Variability Gage Oxide $=\mathrm{Li}_{2} \mathrm{O}(\mathrm{wt} \%)$

Variability Chart for Measured

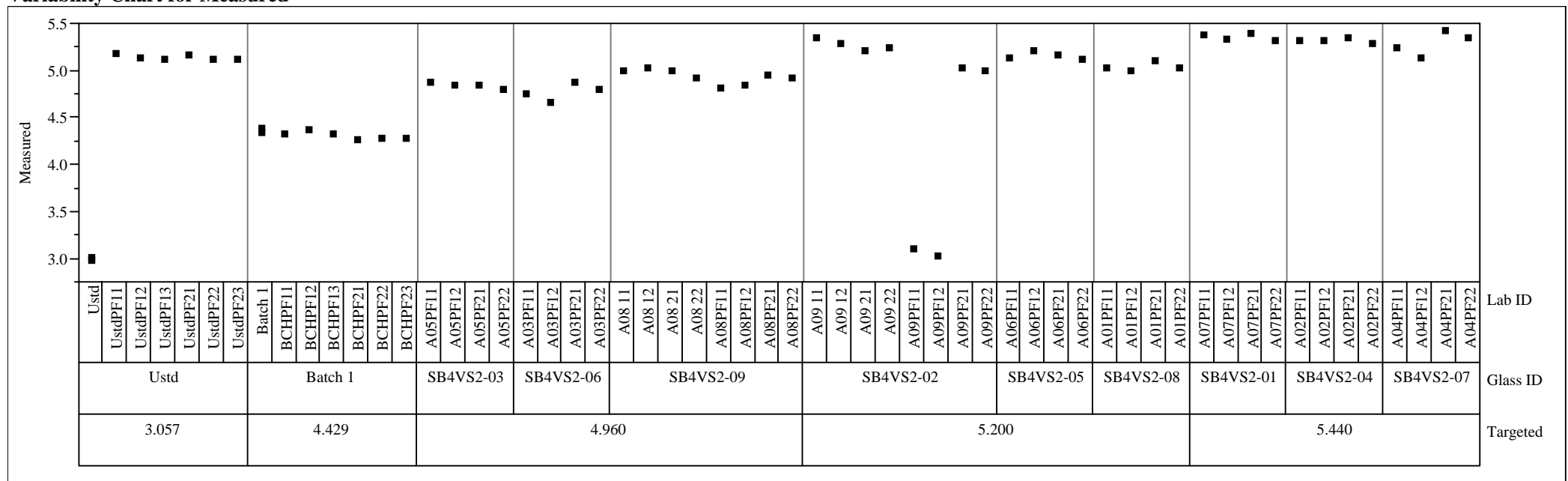

Variability Gage Oxide $=\mathrm{Li}_{2} \mathrm{O}(w t \%)$

Variability Chart for Measured bc

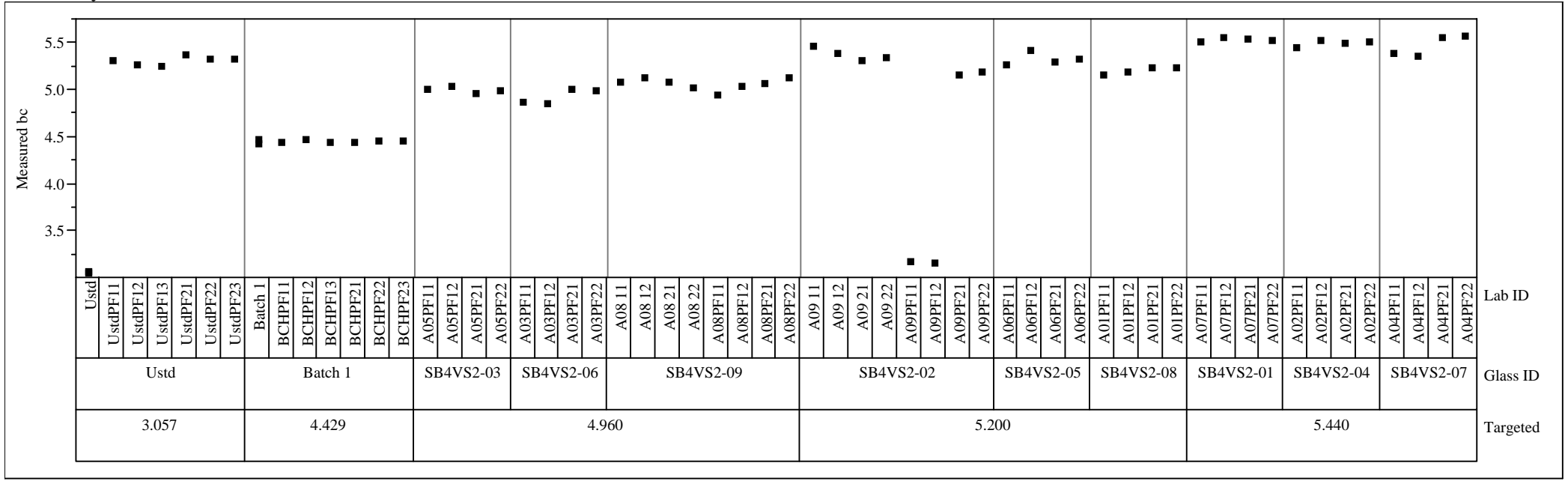


Figure A9. Average Measured and Bias-Corrected (bc) Versus Targeted Compositions by Glass ID by Oxide

Overlay Plot Oxide $=\mathrm{Al}_{2} \mathrm{O}_{3}$ (wt \%)

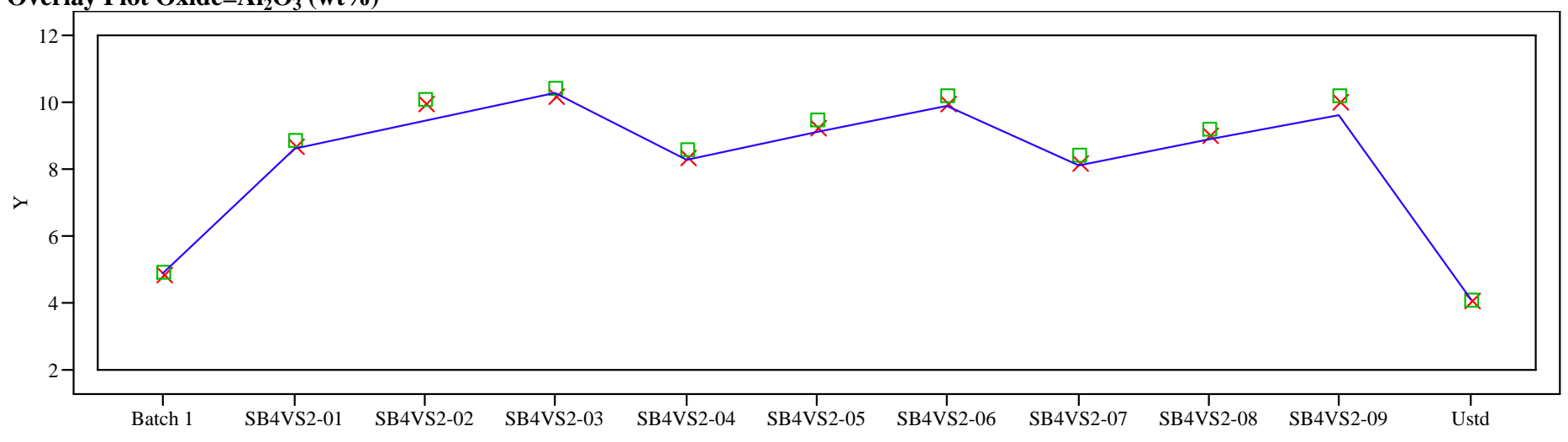

Glass ID

Overlay Plot Oxide $=\mathrm{B}_{2} \mathrm{O}_{3}(\mathrm{wt} \%)$

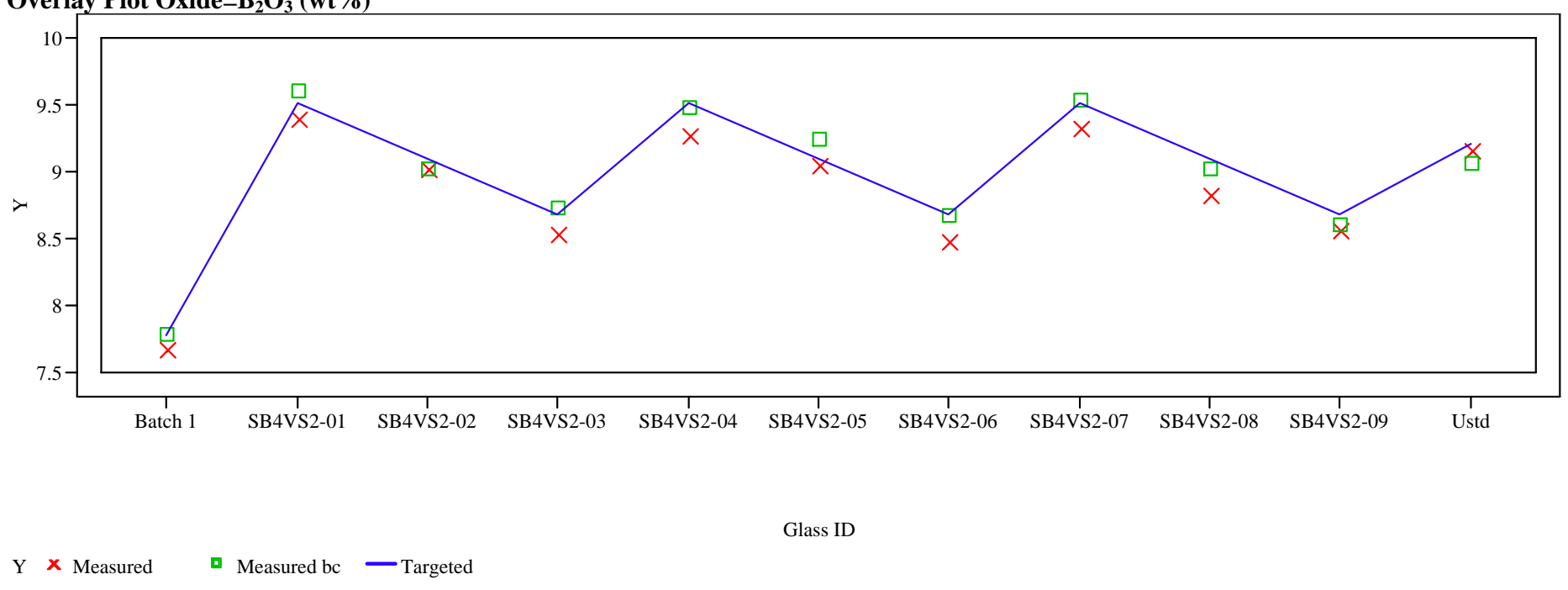


Figure A9. Average Measured and Bias-Corrected (bc) Versus Targeted Compositions by Glass ID by Oxide

Overlay Plot Oxide=BaO (wt \%)

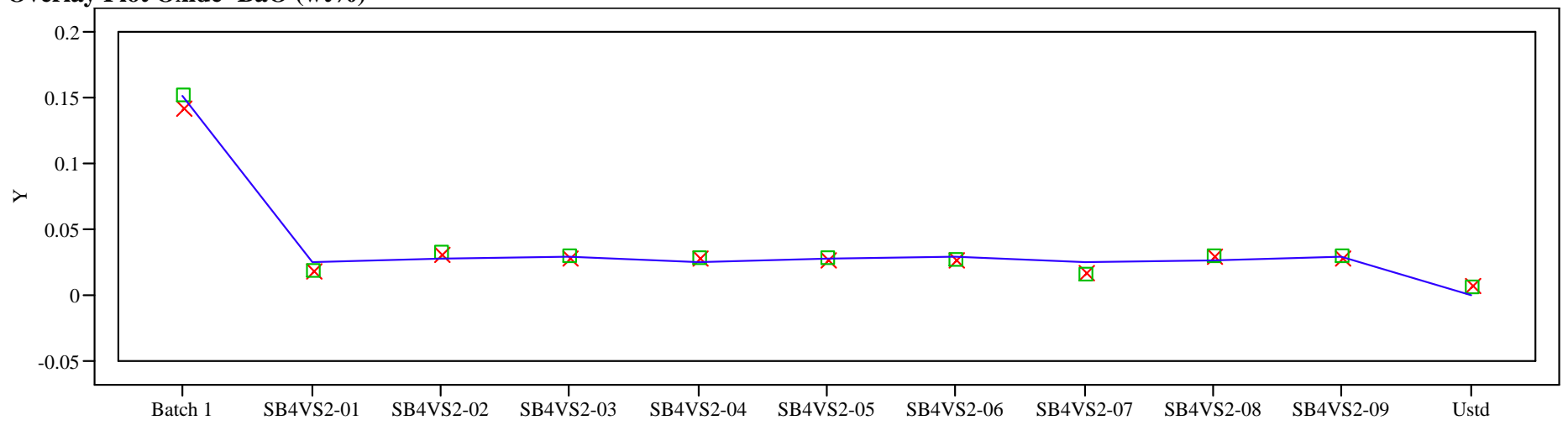

Overlay Plot Oxide $=\mathrm{CaO}(\mathrm{wt} \%)$

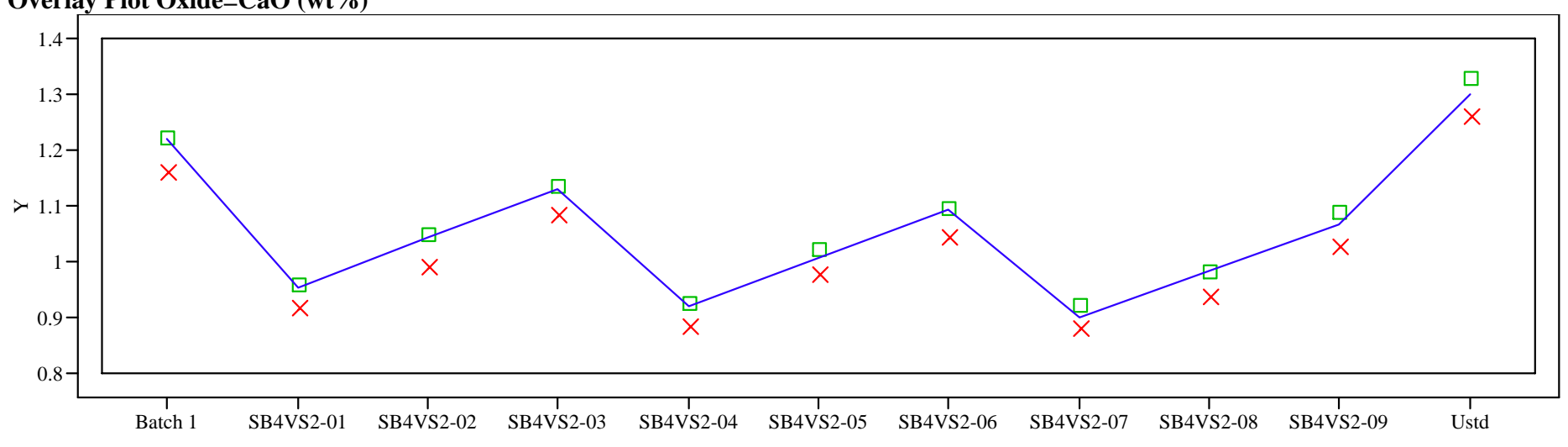

Glass ID

Y $\times$ Measured $\square$ Measured bc — Targeted 
Figure A9. Average Measured and Bias-Corrected (bc) Versus Targeted Compositions by Glass ID by Oxide

Overlay Plot Oxide $=\mathrm{Ce}_{2} \mathrm{O}_{3}$ (wt \%)

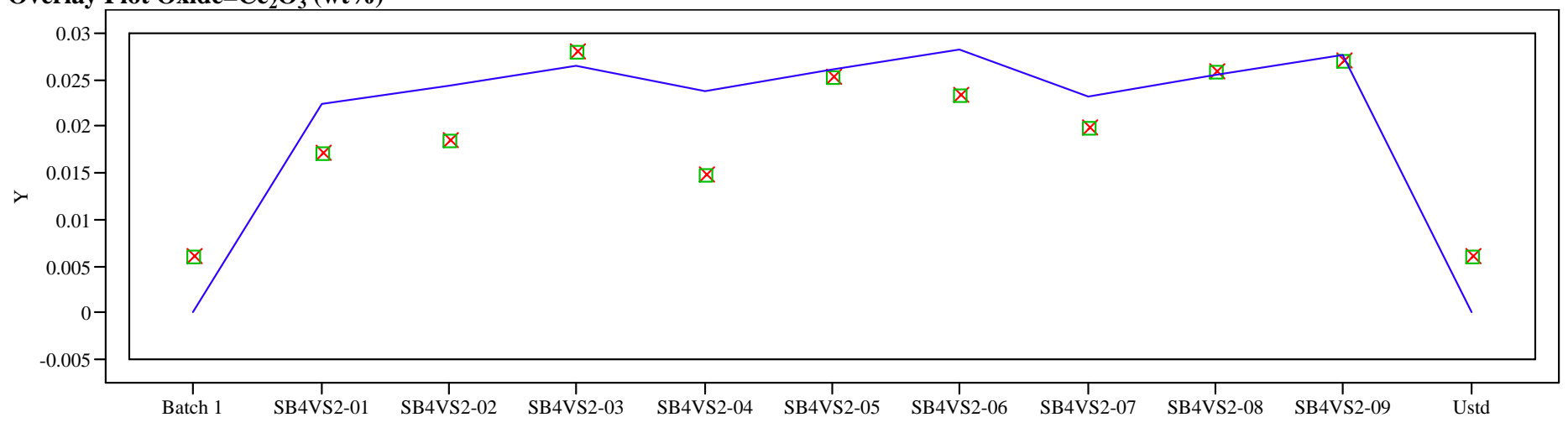

Overlay Plot Oxide $=\mathrm{Cr}_{2} \mathrm{O}_{3}(\mathrm{wt} \%)$

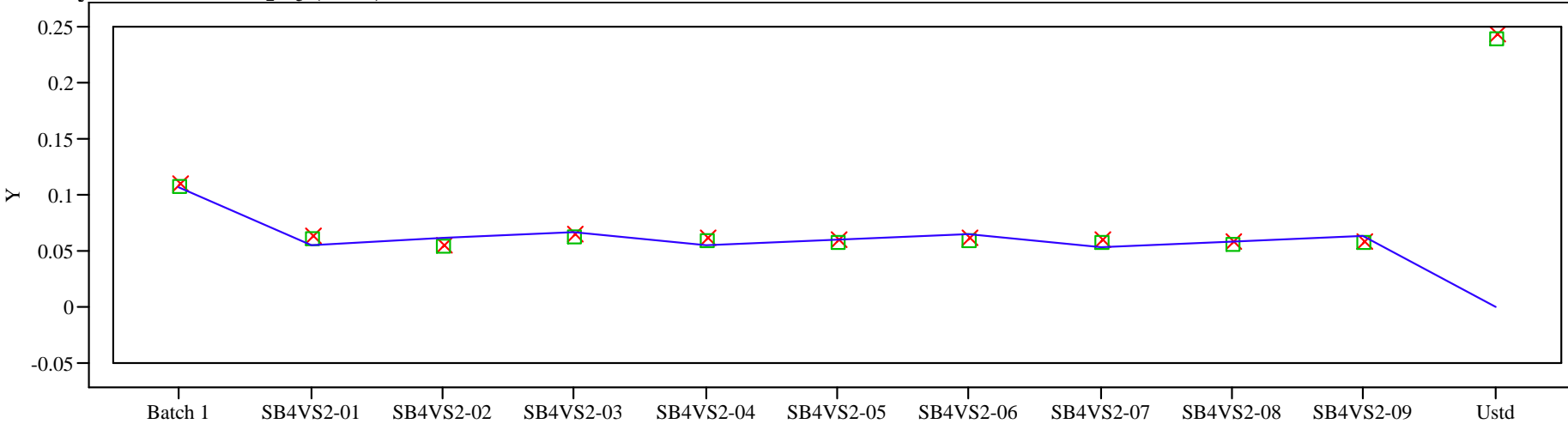
Y $\times$ Measured
- Measured bc — Targeted

Glass ID 
Figure A9. Average Measured and Bias-Corrected (bc) Versus Targeted Compositions by Glass ID by Oxide

Overlay Plot Oxide=CuO (wt\%)
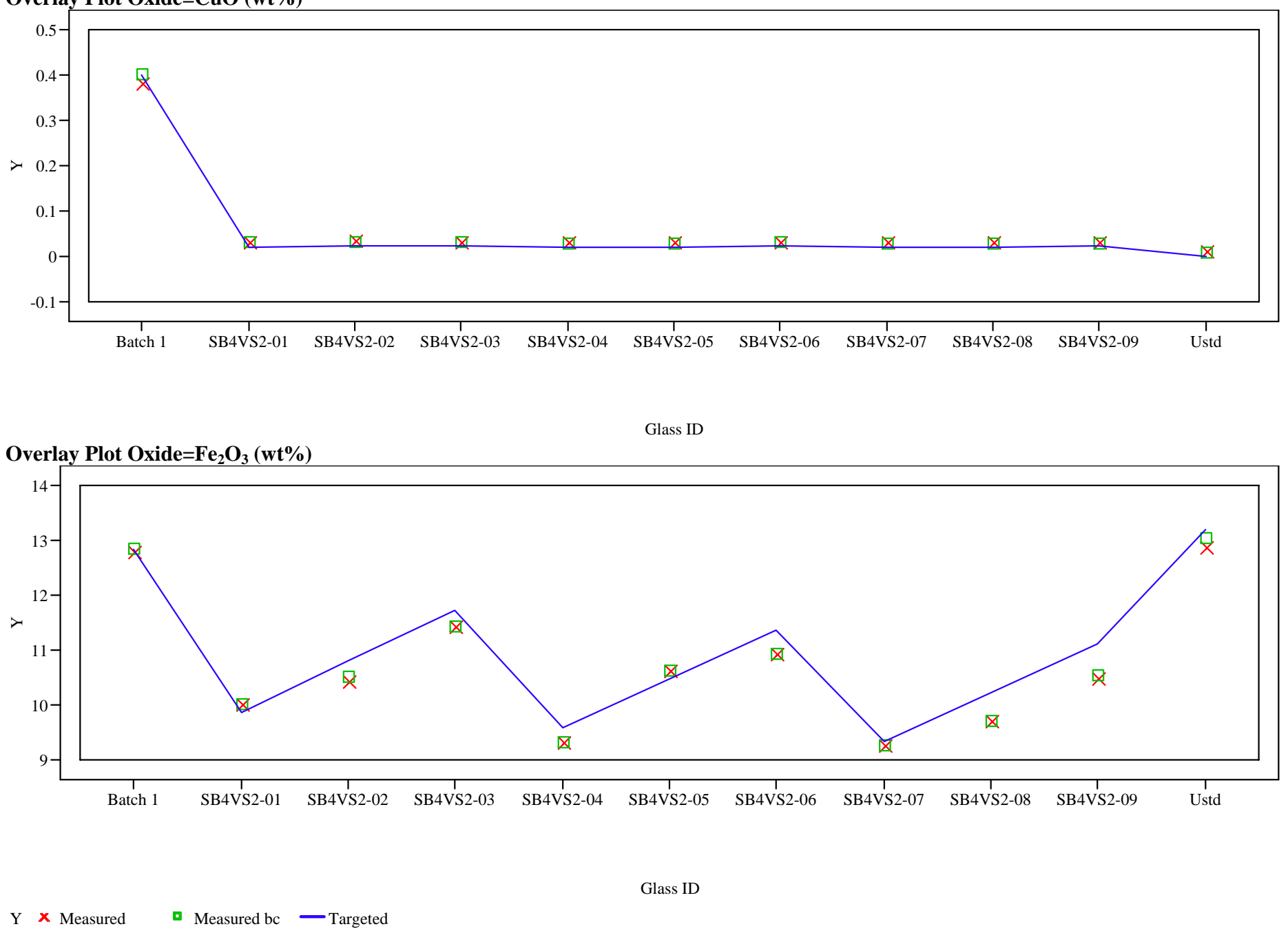
Figure A9. Average Measured and Bias-Corrected (bc) Versus Targeted Compositions by Glass ID by Oxide

Overlay Plot Oxide $=\mathrm{La}_{2} \mathrm{O}_{3}(\mathrm{wt} \%)$

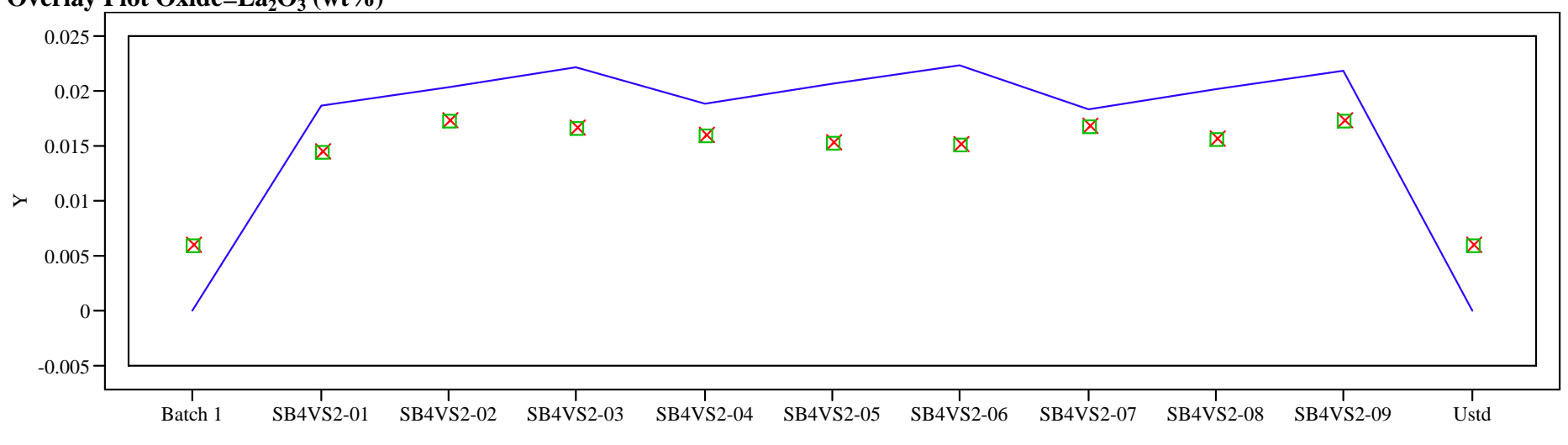

Glass ID

Overlay Plot Oxide $=\mathrm{Li}_{2} \mathrm{O}$ (wt\%)

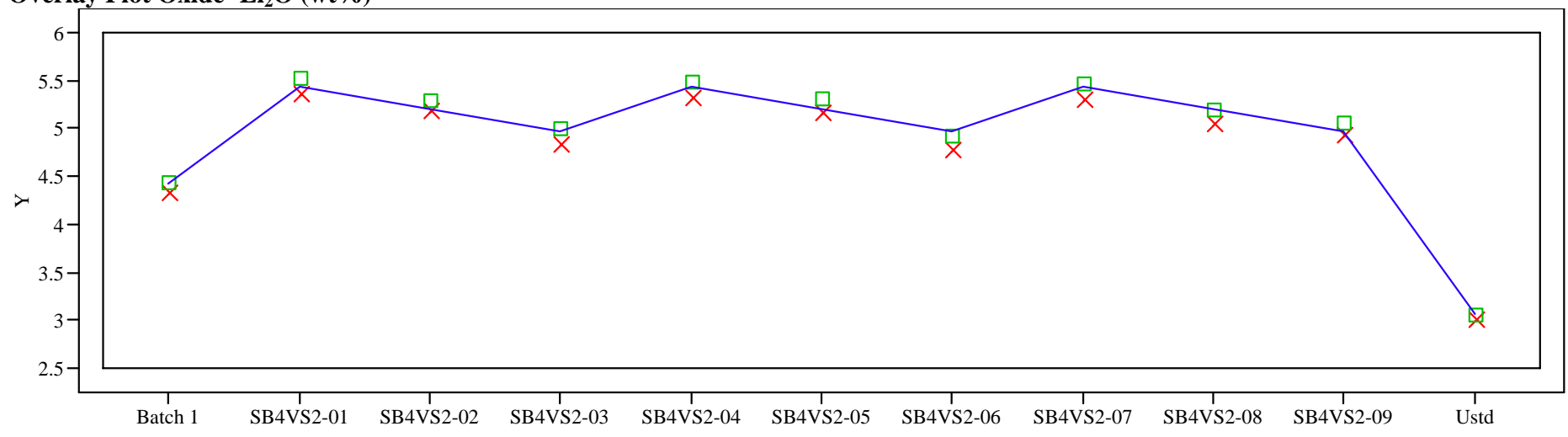

Y $\times$ Measured $\square$ Measured bc — Targeted 
Figure A9. Average Measured and Bias-Corrected (bc) Versus Targeted Compositions by Glass ID by Oxide

Overlay Plot Oxide=MgO (wt \%)

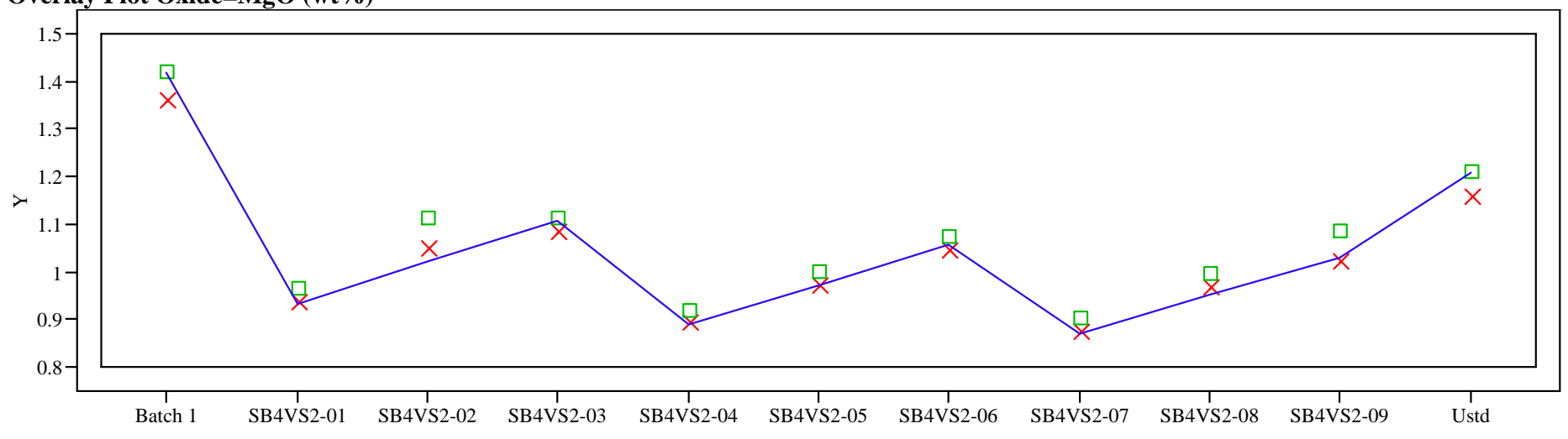

Glass ID

Overlay Plot Oxide $=\mathrm{MnO}($ wt $\%)$

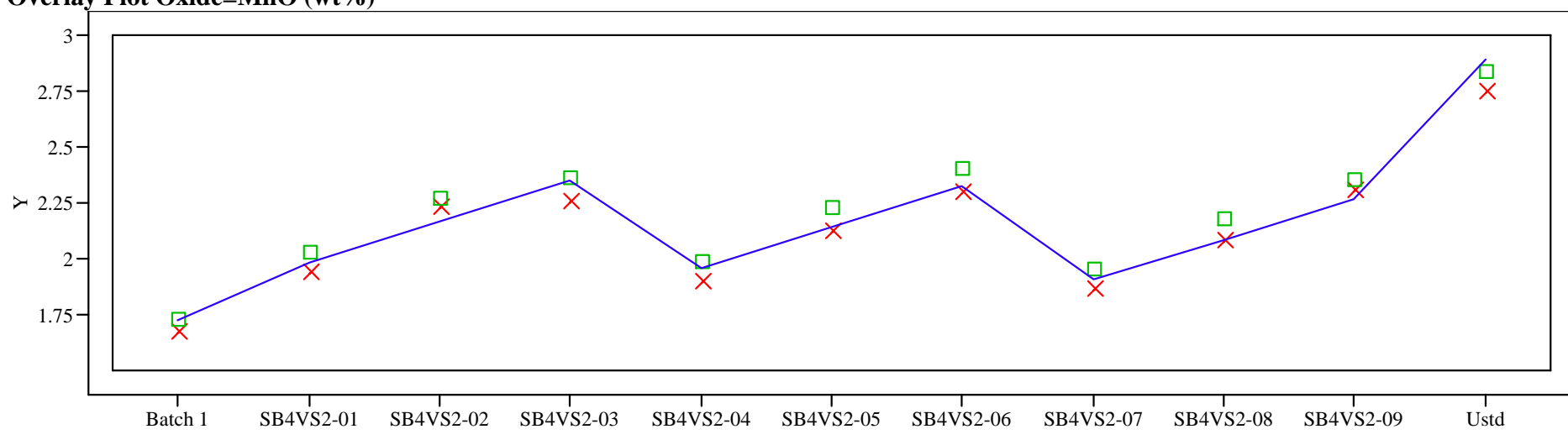

Glass ID

\footnotetext{
Y $\times$ Measured $\square$ Measured bc — Targeted
} 
Figure A9. Average Measured and Bias-Corrected (bc) Versus Targeted Compositions by Glass ID by Oxide

Overlay Plot Oxide $=\mathrm{Na}_{2} \mathrm{O}($ wt $\%)$
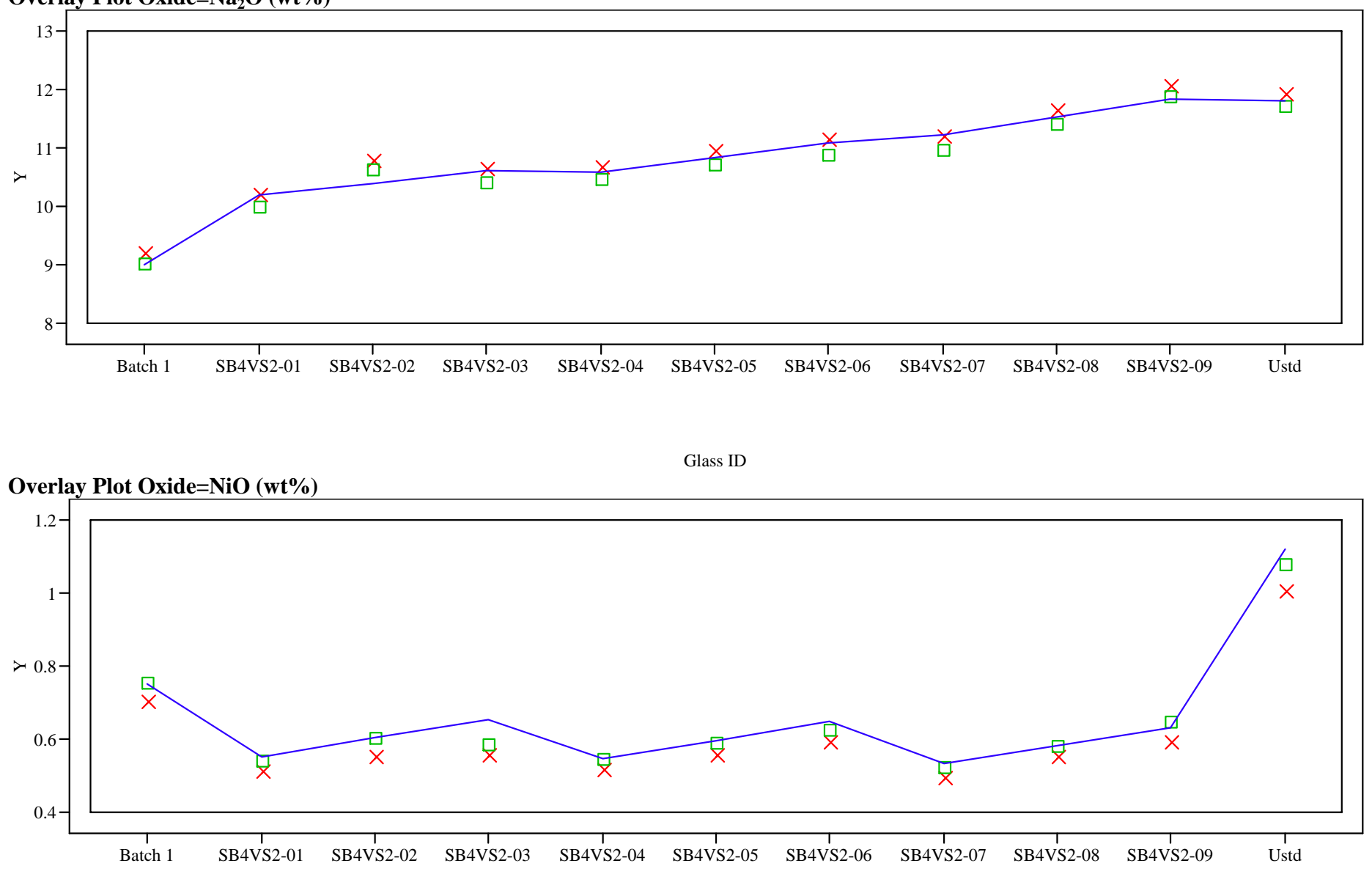

Glass ID

\footnotetext{
Y $\times$ Measured $\square$ Measured bc — Targeted
} 
Figure A9. Average Measured and Bias-Corrected (bc) Versus Targeted Compositions by Glass ID by Oxide

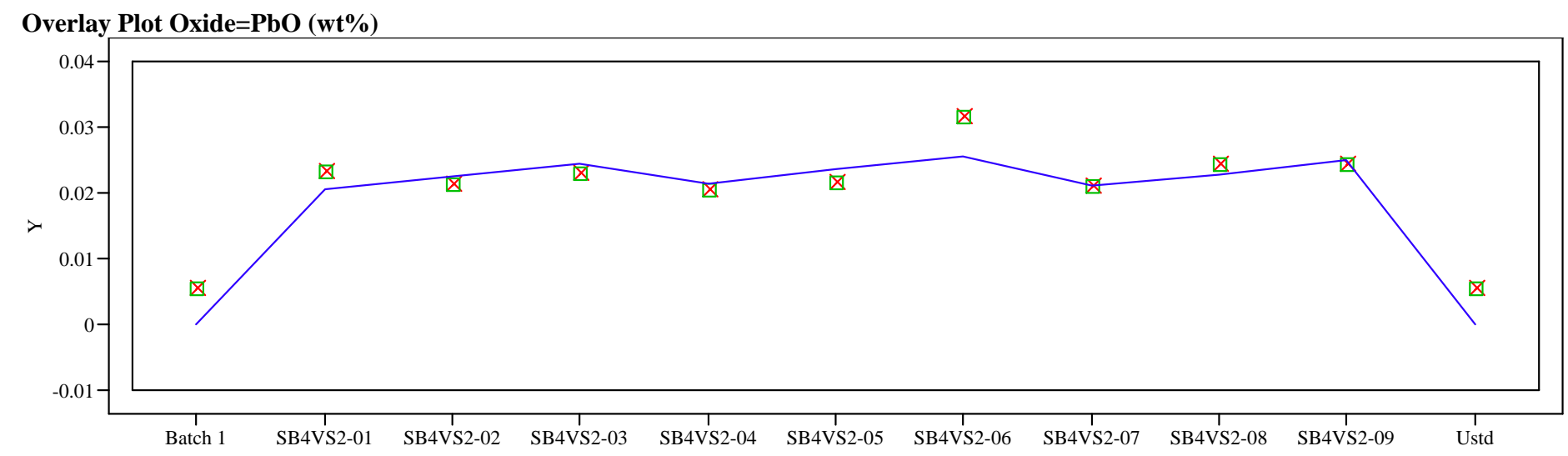

Overlay Plot Oxide $=\mathrm{SiO}_{2}$ (wt \%) Glass ID

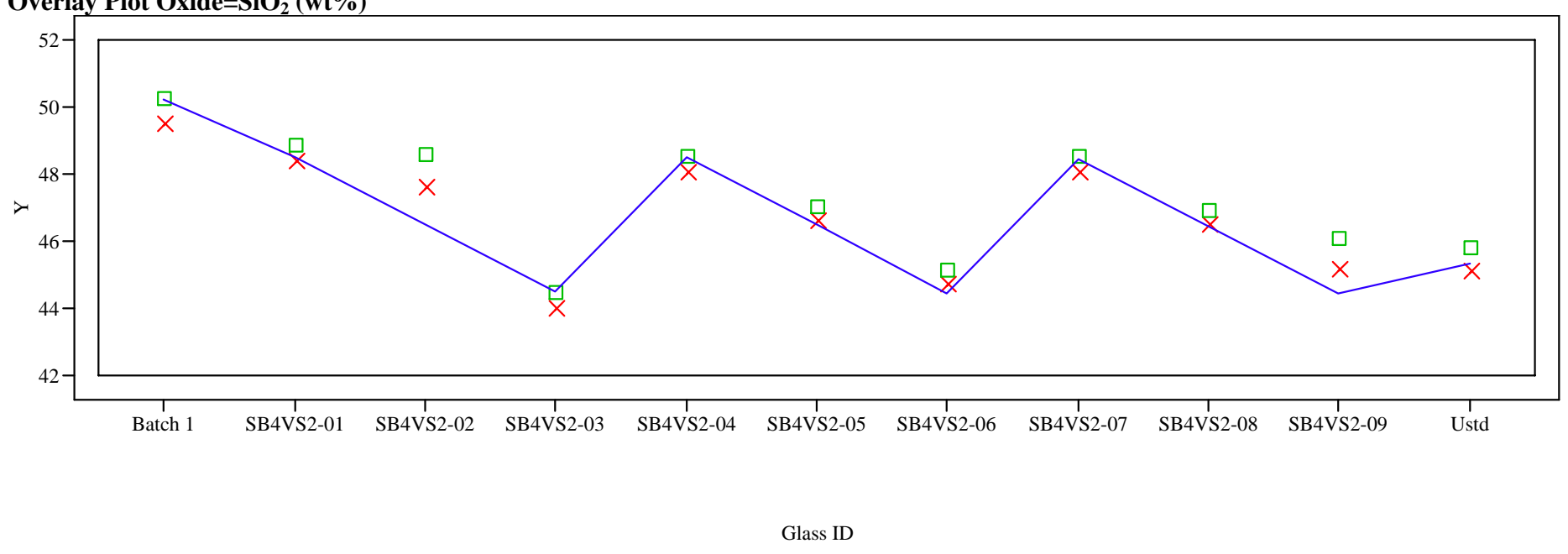

Y $\times$ Measured $\square$ Measured bc -Targeted 
Figure A9. Average Measured and Bias-Corrected (bc) Versus Targeted Compositions by Glass ID by Oxide

Overlay Plot Oxide $=\mathrm{SO}_{4}(\mathrm{wt} \%)$

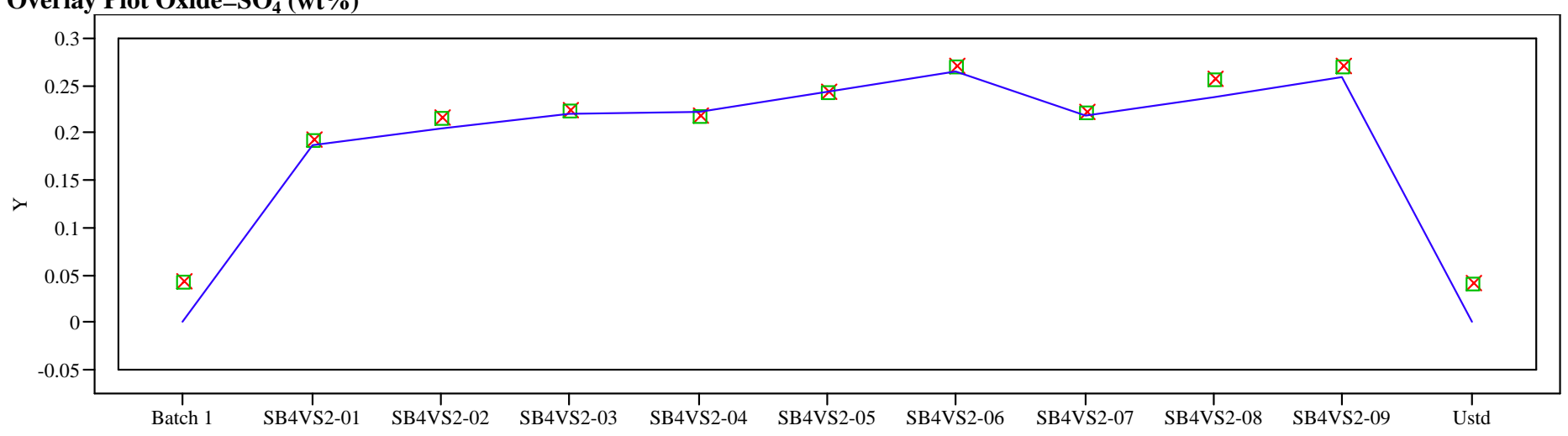

Overlay Plot Oxide $=\mathrm{TiO}_{2}(\mathrm{wt} \%)$

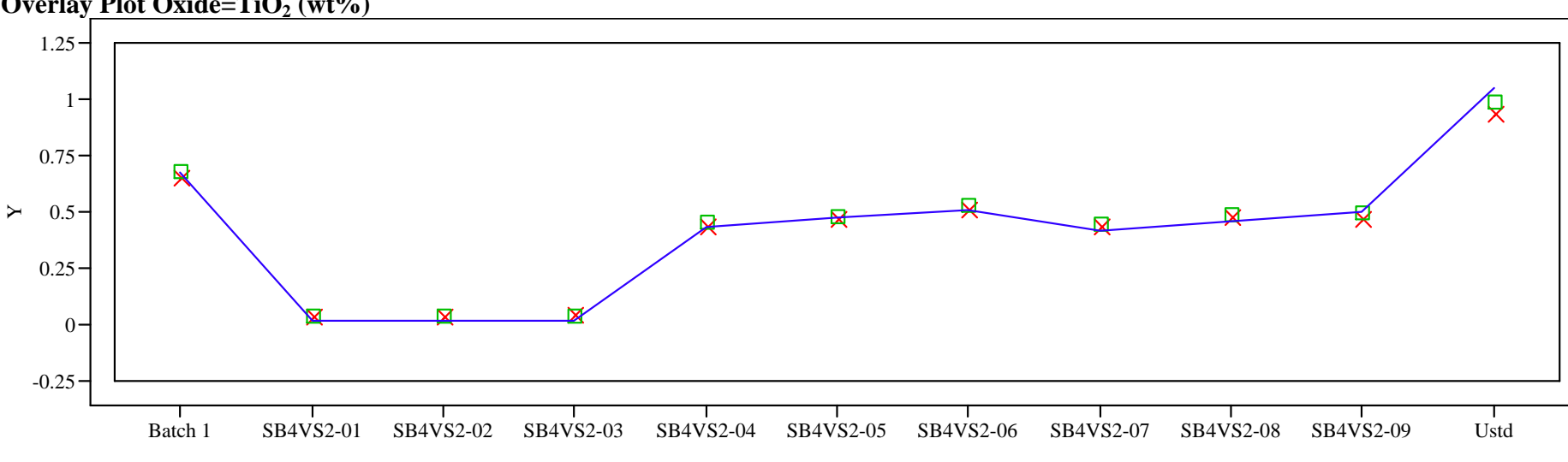

Glass ID

\footnotetext{
Y $\times$ Measured $\square$ Measured bc - Targeted
} 
Figure A9. Average Measured and Bias-Corrected (bc) Versus Targeted Compositions by Glass ID by Oxide

Overlay Plot Oxide $=\mathrm{U}_{3} \mathrm{O}_{8}(\mathrm{wt} \%)$

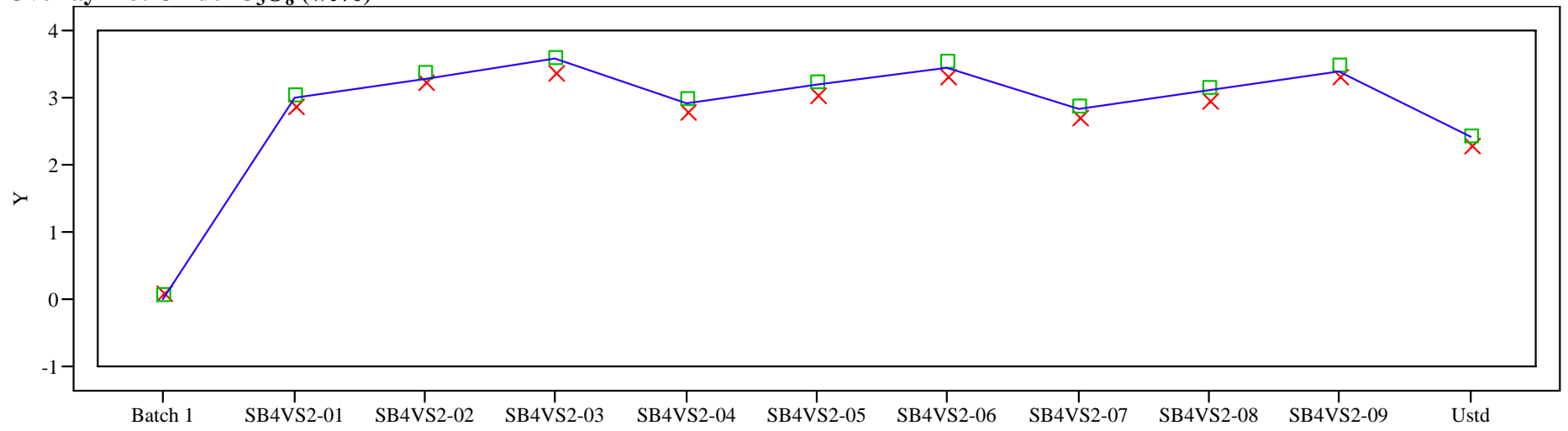

Overlay Plot Oxide $=\mathrm{ZrO}_{2}($ wt $\%) \quad$ Glass ID

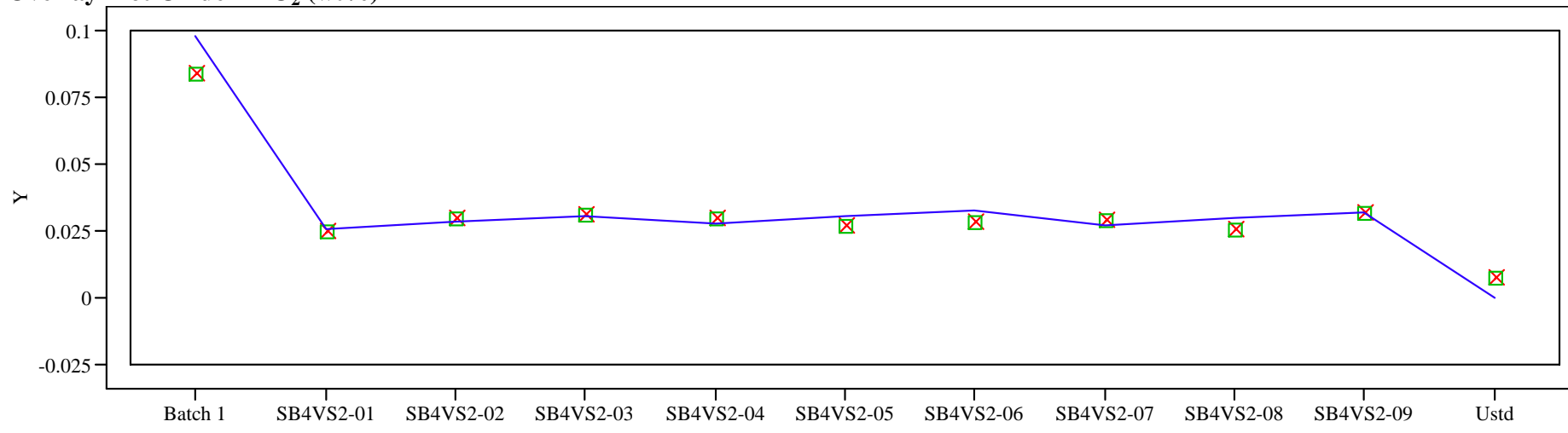

Glass ID

\footnotetext{
$\mathrm{Y} \times$ Measured $\square$ Measured bc - Targeted
} 
Figure A9. Average Measured and Bias-Corrected (bc) Versus Targeted Compositions by Glass ID by Oxide

Overlay Plot Oxide=Sum of Oxides (wt \%)

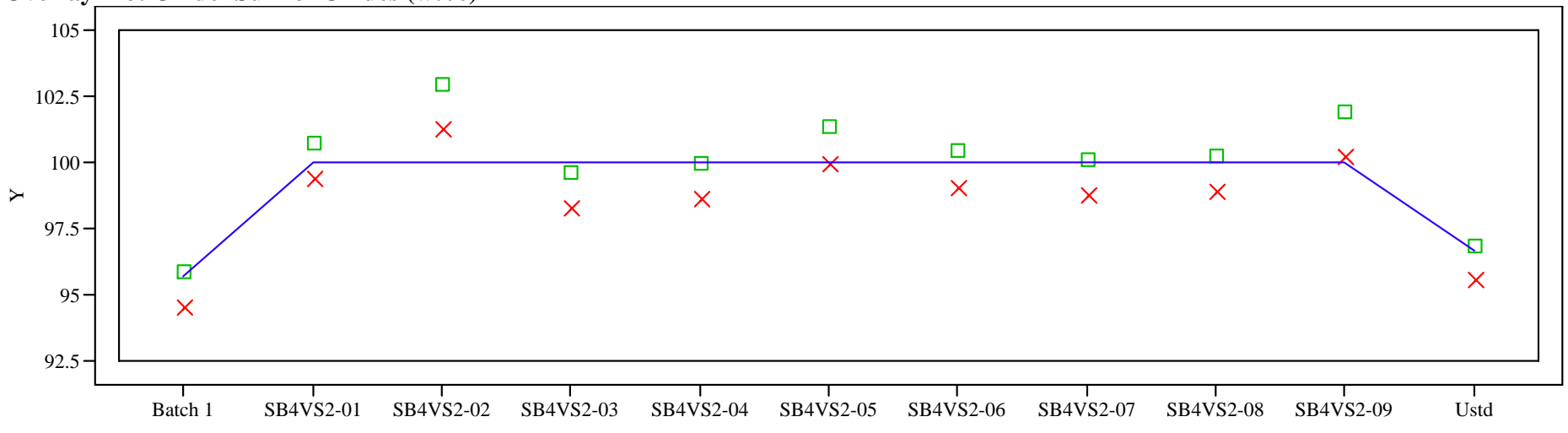

Glass ID

\footnotetext{
$\mathrm{Y} \times$ Measured $\square$ Measured bc - Targeted
} 


\section{Appendix B:}

\section{Tables and Figures Supporting the Analysis of the PCT Results for the SB4 Variability Study Glasses in Support of a Second Decant}


WSRC-STI-2008-00315

Revision 0

This page intentionally left blank. 
Table B1. PSAL Measurements of the PCT Solutions for the Study Glasses AsReceived (ar) and After Appropriate Adjustments (in ppm)

\begin{tabular}{|c|c|c|c|c|c|c|c|c|c|c|c|c|}
\hline Glass ID & $\begin{array}{c}\text { Heat } \\
\text { Treatment }\end{array}$ & Block & Seq & Lab ID & $\mathrm{B}$ ar & $\mathrm{Li}$ ar & $\mathrm{Na}$ ar & Si ar & $\begin{array}{c}\mathrm{B} \\
(\mathrm{ppm})\end{array}$ & $\begin{array}{c}\mathrm{Li} \\
(\mathrm{ppm})\end{array}$ & $\begin{array}{c}\mathrm{Na} \\
(\mathrm{ppm})\end{array}$ & $\begin{array}{c}\mathrm{Si} \\
(\mathrm{ppm})\end{array}$ \\
\hline Soln Std & ref & 1 & 1 & STD-11 & 20.7 & 9.87 & 80.4 & 50.5 & 20.700 & 9.870 & 80.400 & 50.500 \\
\hline SB4VS2-06 & quenched & 1 & 2 & B47 & 12.5 & 11 & 33.9 & 59.6 & 20.834 & 18.334 & 56.501 & 99.335 \\
\hline SB4VS2-04 & CCC & 1 & 3 & B38 & 11.7 & 10.6 & 27 & 61.8 & 19.500 & 17.667 & 45.001 & 103.002 \\
\hline SB4VS2-03 & CCC & 1 & 4 & B33 & 12.5 & 11.3 & 31.7 & 60 & 20.834 & 18.834 & 52.834 & 100.002 \\
\hline SB4VS2-03 & quenched & 1 & 5 & B52 & 12.8 & 11.6 & 32.4 & 62.3 & 21.334 & 19.334 & 54.001 & 103.835 \\
\hline SB4VS2-07 & CCC & 1 & 6 & B34 & 12.5 & 11.4 & 32.2 & 65.4 & 20.834 & 19.000 & 53.668 & 109.002 \\
\hline SB4VS2-09 & $\mathrm{CCC}$ & 1 & 7 & B39 & 11.9 & 10.4 & 37.3 & 59.3 & 19.834 & 17.334 & 62.168 & 98.835 \\
\hline ARM-1 & ref & 1 & 8 & B18 & 11 & 8.72 & 22.9 & 38 & 18.334 & 14.534 & 38.167 & 63.335 \\
\hline SB4VS2-05 & quenched & 1 & 9 & B11 & 11 & 10.3 & 28.2 & 57.3 & 18.334 & 17.167 & 47.001 & 95.502 \\
\hline SB4VS2-04 & quenched & 1 & 10 & B04 & 12 & 11.2 & 27.5 & 63.6 & 20.000 & 18.667 & 45.834 & 106.002 \\
\hline SB4VS2-05 & CCC & 1 & 11 & B42 & 11.8 & 10.7 & 30 & 60.9 & 19.667 & 17.834 & 50.001 & 101.502 \\
\hline SB4VS2-08 & quenched & 1 & 12 & B12 & 12 & 10.9 & 34.6 & 61.6 & 20.000 & 18.167 & 57.668 & 102.669 \\
\hline Soln Std & ref & 1 & 13 & STD-12 & 19.7 & 9.78 & 80.7 & 49.2 & 19.700 & 9.780 & 80.700 & 49.200 \\
\hline SB4VS2-01 & $\overline{\mathrm{CCC}}$ & 1 & 14 & B43 & 12 & 11 & 26.4 & 62.3 & 20.000 & 18.334 & 44.001 & 103.835 \\
\hline EA & ref & 1 & 15 & B17 & 34 & 10.5 & 93 & 50.8 & 566.668 & 175.000 & 1550.003 & 846.668 \\
\hline SB4VS2-02 & CCC & 1 & 16 & B09 & 12.3 & 11.1 & 29.1 & 60.3 & 20.500 & 18.500 & 48.501 & 100.502 \\
\hline SB4VS2-08 & CCC & 1 & 17 & B57 & 11.7 & 10.7 & 34.2 & 61.2 & 19.500 & 17.834 & 57.001 & 102.002 \\
\hline SB4VS2-07 & quenched & 1 & 18 & B05 & 12.6 & 11.7 & 33.3 & 66.2 & 21.000 & 19.500 & 55.501 & 110.336 \\
\hline SB4VS2-01 & quenched & 1 & 19 & B29 & 12.4 & 11.6 & 26.7 & 63.6 & 20.667 & 19.334 & 44.501 & 106.002 \\
\hline SB4VS2-02 & quenched & 1 & 20 & B32 & 12.3 & 11.4 & 28.7 & 61.5 & 20.500 & 19.000 & 47.834 & 102.502 \\
\hline SB4VS2-06 & CCC & 1 & 21 & B27 & 11.7 & 10.5 & 33.1 & 58.6 & 19.500 & 17.500 & 55.168 & 97.669 \\
\hline blank & ref & 1 & 22 & B62 & 0.143 & $<1.00$ & $<0.100$ & $<0.100$ & 0.238 & 0.833 & 0.083 & 0.083 \\
\hline SB4VS2-09 & quenched & 1 & 23 & B56 & 11.9 & 10.8 & 38.6 & 59.5 & 19.834 & 18.000 & 64.335 & 99.169 \\
\hline Soln Std & ref & 1 & 24 & STD-13 & 19.9 & 9.85 & 83.2 & 49.6 & 19.900 & 9.850 & 83.200 & 49.600 \\
\hline Soln Std & ref & 2 & 1 & STD-21 & 19.6 & 9.37 & 76.8 & 48.4 & 19.600 & 9.370 & 76.800 & 48.400 \\
\hline SB4VS2-08 & quenched & 2 & 2 & B08 & 11.4 & 10.4 & 32.9 & 58.4 & 19.000 & 17.334 & 54.834 & 97.335 \\
\hline SB4VS2-06 & CCC & 2 & 3 & B54 & 11.2 & 10.1 & 31.4 & 56.9 & 18.667 & 16.834 & 52.334 & 94.835 \\
\hline ARM-1 & ref & 2 & 4 & B19 & 9.7 & 7.93 & 20.4 & 35.4 & 16.167 & 13.217 & 34.001 & 59.001 \\
\hline SB4VS2-07 & CCC & 2 & 5 & B20 & 11.6 & 11 & 31.4 & 63.7 & 19.334 & 18.334 & 52.334 & 106.169 \\
\hline SB4VS2-01 & quenched & 2 & 6 & B13 & 11.5 & 10.9 & 24.7 & 61.7 & 19.167 & 18.167 & 41.167 & 102.835 \\
\hline SB4VS2-05 & CCC & 2 & 7 & B46 & 10.5 & 9.76 & 27.3 & 57.7 & 17.500 & 16.267 & 45.501 & 96.169 \\
\hline SB4VS2-02 & quenched & 2 & 8 & $\mathrm{~B} 40$ & 11.4 & 10.8 & 26.2 & 60.5 & 19.000 & 18.000 & 43.668 & 100.835 \\
\hline SB4VS2-01 & CCC & 2 & 9 & B50 & 11.3 & 10.7 & 24.9 & 62 & 18.834 & 17.834 & 41.501 & 103.335 \\
\hline SB4VS2-07 & quenched & 2 & 10 & B21 & & 11 & 29.8 & 64.3 & 19.667 & 18.334 & 49.668 & 107.169 \\
\hline SB4VS2-06 & quenched & 2 & 11 & B36 & & 10.5 & 31.2 & 59.4 & 19.167 & 17.500 & 52. & 99.002 \\
\hline SB4VS2-04 & CCC & 2 & 12 & B48 & .1 & 10.3 & 25.5 & 63 & 18.500 & 17.167 & 42.5 & 105.002 \\
\hline Soln Std & ref & 2 & 13 & STD-22 & 19.6 & 9.65 & 77.9 & 50.9 & 19.600 & 9.650 & 77.900 & 50.900 \\
\hline SB4VS2-03 & CCC & 2 & 14 & B01 & 11.2 & 10.3 & 29.1 & 56.9 & 18.667 & 17.167 & 48.501 & 94.835 \\
\hline SB4VS2-09 & quenched & 2 & 15 & B53 & 11.5 & 10.3 & 36.2 & 59.3 & 19.167 & 17.167 & 60.335 & 98.835 \\
\hline SB4VS2-04 & quenched & 2 & 16 & B61 & 11.2 & 10.8 & 28.7 & 63.4 & 18.667 & 18.000 & 47.834 & 105.669 \\
\hline SB4VS2-09 & CCC & 2 & 17 & B35 & 11.3 & 10.2 & 36.3 & 58 & 18.834 & 17.000 & 60.501 & 96.669 \\
\hline EA & ref & 2 & 18 & B26 & 33.9 & 10.4 & 94 & 51.3 & 565.001 & 173.334 & 1566.670 & 855.002 \\
\hline SB4VS2-02 & CCC & 2 & 19 & B03 & 11.6 & 10.7 & 28.5 & 59.6 & 19.334 & 17.834 & 47.501 & 99.335 \\
\hline SB4VS2-08 & CCC & 2 & 20 & B49 & 10.9 & 10.1 & 33.6 & 59.3 & 18.167 & 16.834 & 56.001 & 98.835 \\
\hline SB4VS2-03 & quenched & 2 & 21 & B30 & 11.9 & 11.2 & 32.7 & 59 & 19.834 & 18.667 & 54.501 & 98.335 \\
\hline SB4VS2-05 & quenched & 2 & 22 & B02 & 10.3 & 10 & 28.8 & 55.8 & 17.167 & 16.667 & 48.0 & 93.002 \\
\hline Soln Std & ref & 2 & 23 & STD-23 & & 9.49 & 78 & 48.5 & & 9.490 & & 48.500 \\
\hline Soln Std & ref & 3 & 1 & STD-31 & 19.6 & 9.46 & 78.7 & 48.6 & 19.600 & 9.460 & 78.700 & 48.600 \\
\hline SB4VS2-04 & CCC & 3 & 2 & B60 & 11.4 & 10.3 & 25.9 & 60.9 & 19.000 & 17.167 & 43.168 & 101.502 \\
\hline SB4VS2-07 & quenched & 3 & 3 & B59 & 12.3 & 11.3 & 31.7 & 64.1 & 20.500 & 18.834 & 52.834 & 106.835 \\
\hline SB4VS2-01 & quenched & 3 & 4 & B44 & 11.7 & 11 & 24.8 & 61.1 & 19.500 & 18.334 & 41.334 & 101.835 \\
\hline SB4VS2-05 & CCC & 3 & 5 & B15 & 11 & 10.1 & 29 & 58.9 & 18.334 & 16.834 & 48.334 & 98.169 \\
\hline SB4VS2-07 & $\mathrm{CCC}$ & 3 & 6 & B41 & 11.3 & 10.5 & 30.7 & 60.3 & 18.834 & 17.500 & 51.168 & 100.502 \\
\hline SB4VS2-03 & quenched & 3 & 7 & B58 & 11.8 & 11 & 32.1 & 57.3 & 19.667 & 18.334 & 53.501 & 95.502 \\
\hline SB4VS2-09 & $\mathrm{CCC}$ & 3 & 8 & B55 & 10.9 & 10 & 36 & 56.4 & 18.167 & 16.667 & 60.001 & 94.002 \\
\hline SB4VS2-03 & CCC & 3 & 9 & B28 & 11.6 & 10.7 & 32 & 57.4 & 19.334 & 17.834 & 53.334 & 95.669 \\
\hline SB4VS2-08 & quenched & 3 & 10 & B51 & 11.4 & 10.7 & 34.8 & 59.5 & 19.000 & 17.834 & 58.001 & 99.169 \\
\hline SB4VS2-06 & quenched & 3 & 11 & B31 & 11.4 & 10.5 & 34.4 & 57.8 & 19.000 & 17.500 & 57.334 & 96.335 \\
\hline
\end{tabular}


Table B1. PSAL Measurements of the PCT Solutions for the Study Glasses AsReceived (ar) and After Appropriate Adjustments (in ppm)

\begin{tabular}{||c|c|c|c|c|c|c|c|c|c|c|c|c||}
\hline \hline Glass ID & $\begin{array}{c}\text { Heat } \\
\text { Treatment }\end{array}$ & Block & Seq & Lab ID & B ar & Li ar & Na ar & Si ar & $\begin{array}{c}\text { B } \\
(\mathrm{ppm})\end{array}$ & $\begin{array}{c}\text { Li } \\
(\mathrm{ppm})\end{array}$ & $\begin{array}{c}\text { Na } \\
(\mathrm{ppm})\end{array}$ & $\begin{array}{c}\text { Si } \\
(\mathrm{ppm})\end{array}$ \\
\hline SB4VS2-06 & cCC & 3 & 12 & B10 & 11 & 10.3 & 33 & 56.3 & 18.334 & 17.167 & 55.001 & 93.835 \\
\hline Soln Std & ref & 3 & 13 & STD-32 & 18.6 & 9.5 & 82 & 47.7 & 18.600 & 9.500 & 82.000 & 47.700 \\
\hline SB4VS2-09 & quenched & 3 & 14 & B23 & 11.8 & 10.4 & 38.8 & 57.3 & 19.667 & 17.334 & 64.668 & 95.502 \\
\hline EA & ref & 3 & 15 & B07 & 33.5 & 10.4 & 96 & 50.4 & 558.334 & 173.334 & 1600.003 & 840.002 \\
\hline SB4VS2-05 & quenched & 3 & 16 & B22 & 11.6 & 10.7 & 30.7 & 58.7 & 19.334 & 17.834 & 51.168 & 97.835 \\
\hline SB4VS2-01 & cCC & 3 & 17 & B24 & 11.4 & 10.6 & 26.1 & 60.1 & 19.000 & 17.667 & 43.501 & 100.169 \\
\hline SB4VS2-02 & quenched & 3 & 18 & B06 & 11.2 & 10.8 & 28.7 & 57.7 & 18.667 & 18.000 & 47.834 & 96.169 \\
\hline ARM-1 & ref & 3 & 19 & B37 & 9.84 & 8.13 & 22 & 35.4 & 16.400 & 13.550 & 36.667 & 59.001 \\
\hline blank & ref & 3 & 20 & B16 & 0.123 & $<1.00$ & $<0.100$ & $<0.100$ & 0.205 & 0.833 & 0.083 & 0.083 \\
\hline SB4VS2-04 & quenched & 3 & 21 & B45 & 10.7 & 10.6 & 27.9 & 58.9 & 17.834 & 17.667 & 46.501 & 98.169 \\
\hline SB4VS2-02 & cCC & 3 & 22 & B25 & 11.5 & 10.7 & 28.6 & 59.1 & 19.167 & 17.834 & 47.668 & 98.502 \\
\hline SB4VS2-08 & cCC & 3 & 23 & B14 & 11.2 & 10.4 & 34.1 & 59.3 & 18.667 & 17.334 & 56.834 & 98.835 \\
\hline Soln Std & ref & 3 & 24 & STD-33 & 18.7 & 9.62 & 82.1 & 48.1 & 18.700 & 9.620 & 82.100 & 48.100 \\
\hline
\end{tabular}


Figure B1. Laboratory PCT Measurements in Analytical Sequence for Study Glasses with and without Other Results from the Analytical Plans

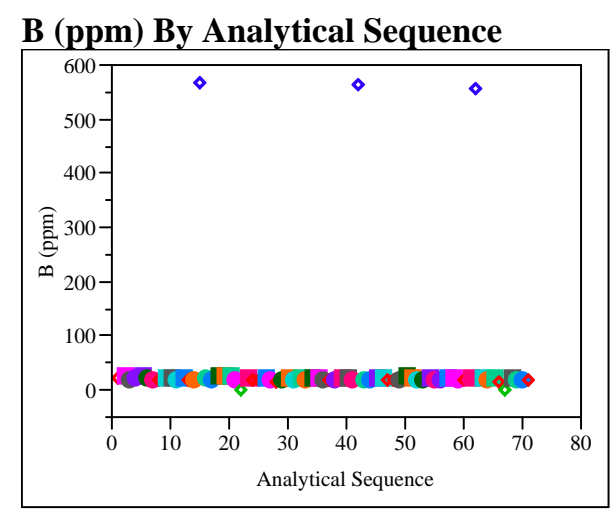

\section{Li (ppm) By Analytical Sequence}

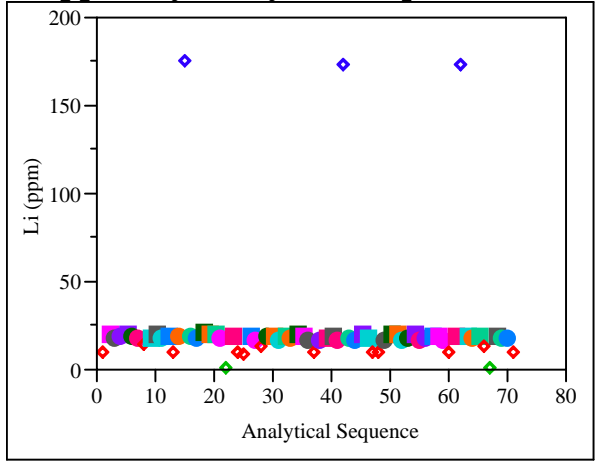

Na (ppm) By Analytical Sequence

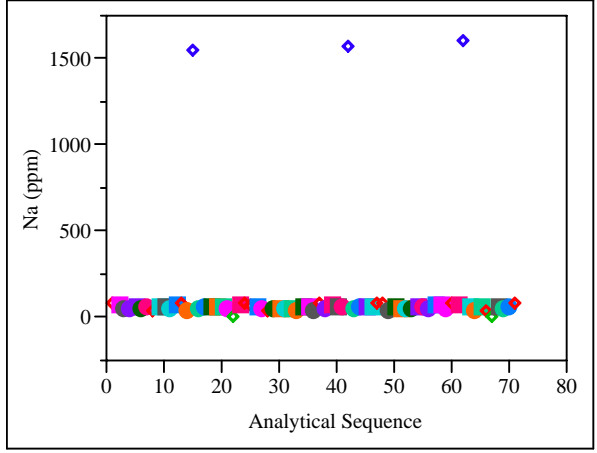

Si (ppm) By Analytical Sequence

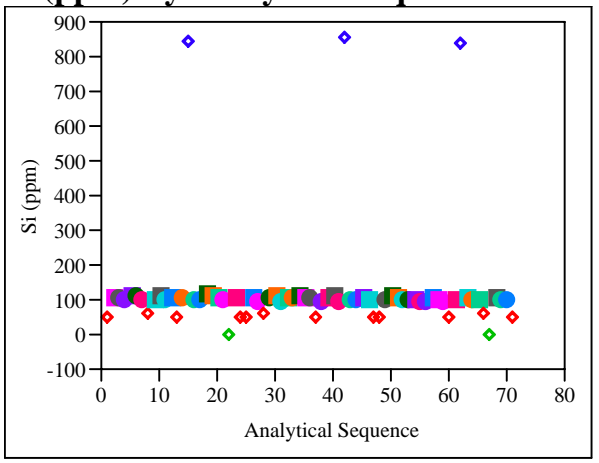

B (ppm) By Analytical Sequence

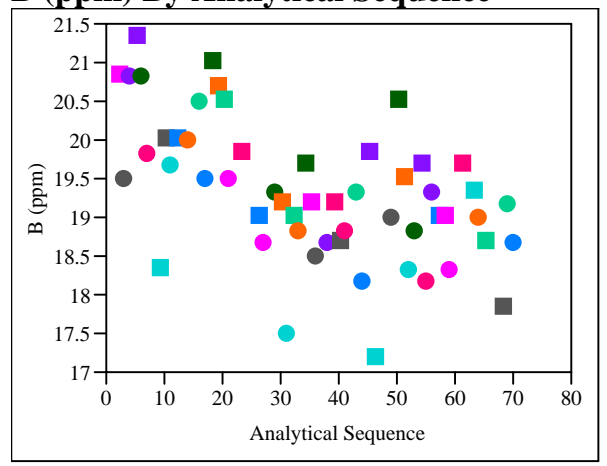

Li (ppm) By Analytical Sequence

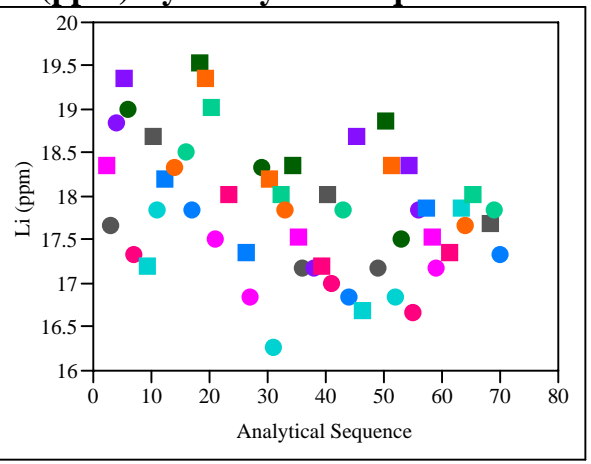

Na (ppm) By Analytical Sequence

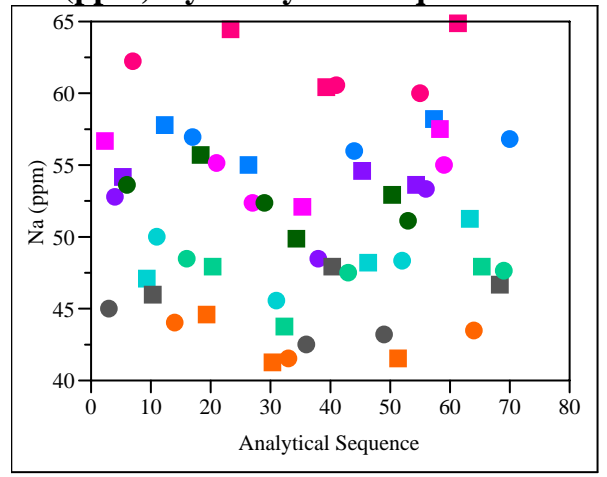

Si (ppm) By Analytical Sequence

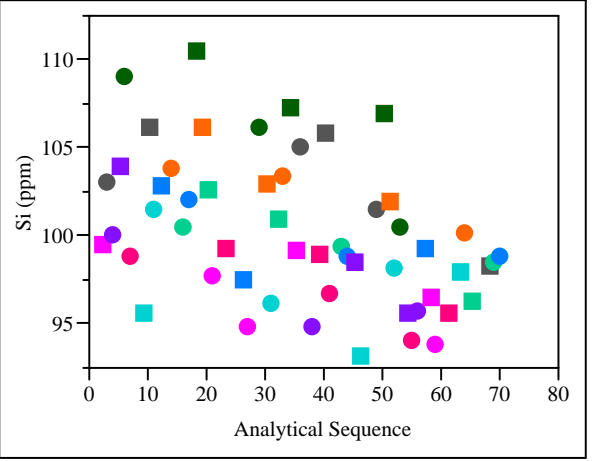


WSRC-STI-2008-00315

Revision 0

Figure B2. Measurements of the Multi-Element Solution Standard by ICP Block

Oneway Analysis of B (ppm) By Block

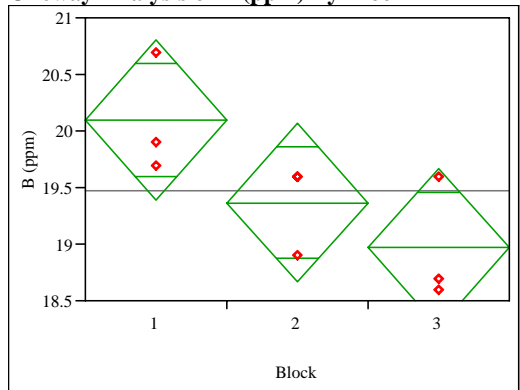

Oneway Anova

Summary of Fit

Rsquare

Adj Rsquare

0.570332

Root Mean Square Error $\quad 0.498888$

Mean of Response

Observations (or Sum Wgts)

Analysis of Variance

Source DF Sum of Squares Mean Square F Ratio Prob > F

$\begin{array}{lrrrrr}\text { Block } & 2 & 1.9822222 & 0.991111 & 3.9821 & 0.0793\end{array}$

Error $\quad 6 \quad 1.4933333$

C. Total $8 \quad 3.4755556$

Means for Oneway Anova

Level Number Mean Std Error Lower 95\% Upper 95\%

$\begin{array}{lllllr}1 & 3 & 20.1000 & 0.28803 & 19.395 & 20.805\end{array}$

$\begin{array}{llllll}1 & 3 & 19.3667 & 0.28803 & 18.662 & 20.071\end{array}$

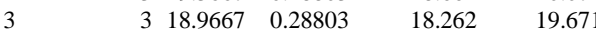

Std Error uses a pooled estimate of error variance

Oneway Analysis of Li (ppm) By Block

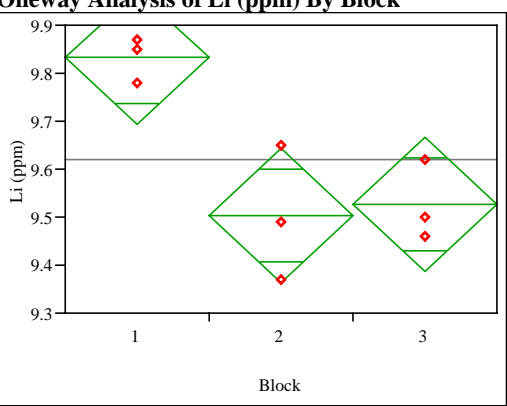

Oneway Anova

Summary of Fit

Rsquare $\quad 0.778789$

Adj Rsquare $\quad 0.705052$

Root Mean Square Error $\quad 0.09815$

Mean of Response 9.62111

Observations (or Sum Wgts)

Analysis of Variance

Source DF Sum of Squares Mean Square F Ratio Prob $>$ F

$\begin{array}{lrrrrr}\text { Block } & 2 & 0.20348889 & 0.101744 & 10.5617 & 0.0108\end{array}$

Error $\quad 6 \quad 0.05780000$

C. Total $8 \quad 0.26128889$

Means for Oneway Anova

Level Number Mean Std Error Lower 95\% Upper 95\%

$\begin{array}{lllrrr}1 & 3 & 9.83333 & 0.05667 & 9.6947 & 9.9720\end{array}$

$\begin{array}{llllll}1 & 3 & 9.50333 & 0.05667 & 9.3647 & 9.6420\end{array}$

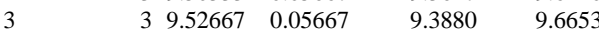

Std Error uses a pooled estimate of error variance
Oneway Analysis of Na (ppm) By Block

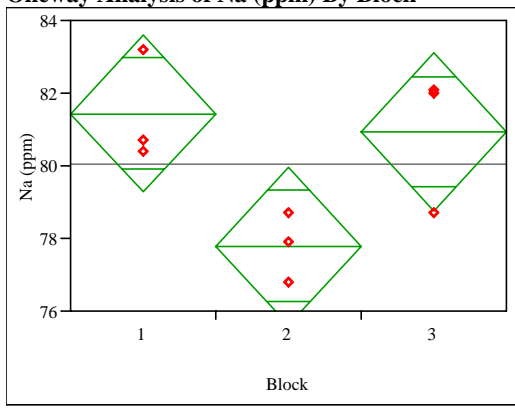

Oneway Anova

Summary of Fit

$\begin{array}{lr}\text { Rsquare } & 0.623794 \\ \text { Adj Rsquare } & 0.498392 \\ \text { Root Mean Square Error } & 1.529343 \\ \text { Mean of Response } & 80.05556 \\ \text { Observations (or Sum Wgts) } & 9\end{array}$

Analysis of Variance

Source DF Sum of Squares Mean Square F Ratio Prob > F

$\begin{array}{llllll}\text { Block } & 2 & 23.268889 & 11.6344 & 4.9743 & 0.0532\end{array}$

$\begin{array}{lllr}\text { Error } & 6 & 14.033333 & 2.3389\end{array}$

C. Total $8 \quad 37.302222$

Means for Oneway Anova

Level Number Mean Std Error Lower 95\% Upper 95\%

$\begin{array}{lrrrrr}1 & 3 & 81.4333 & 0.88297 & 79.273 & 83.594\end{array}$

$\begin{array}{llllll}2 & 3 & 77.8000 & 0.88297 & 75.639 & 79.961\end{array}$

$\begin{array}{llllll}3 & 3 & 80.9333 & 0.88297 & 78.773 & 83.094\end{array}$

Std Error uses a pooled estimate of error variance

Oneway Analysis of Si (ppm) By Block

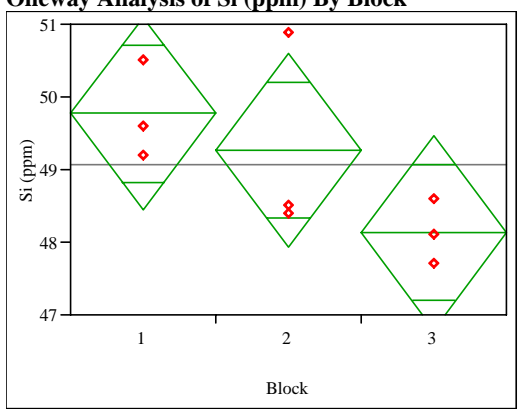

Oneway Anova

Summary of Fit

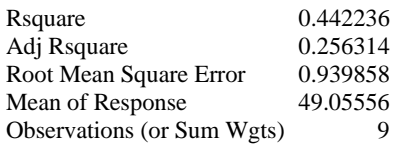

Analysis of Variance

Source DF Sum of Squares Mean Square F Ratio Prob $>$ F

$\begin{array}{llllll}\text { Block } & 2 & 4.2022222 & 2.10111 & 2.3786 & 0.1735\end{array}$

$\begin{array}{llll}\text { Error } & 6 & 5.3000000 & 0.88333\end{array}$

C. Total $8 \quad 9.5022222$

Means for Oneway Anova

Level Number Mean Std Error Lower 95\% Upper 95\%

$\begin{array}{lllllr}1 & 3 & 49.7667 & 0.54263 & 48.439 & 51.094\end{array}$

$\begin{array}{llllll}2 & 3 & 49.2667 & 0.54263 & 47.939 & 50.594\end{array}$

$\begin{array}{lllll}3 & 3 & 48.1333 & 0.54263 & 46.806\end{array} 49.461$

Std Error uses a pooled estimate of error variance 
Figure B3. Laboratory PCT Measurements by Glass Identifier for Study Glasses and Standards

Variability Chart for B (ppm)

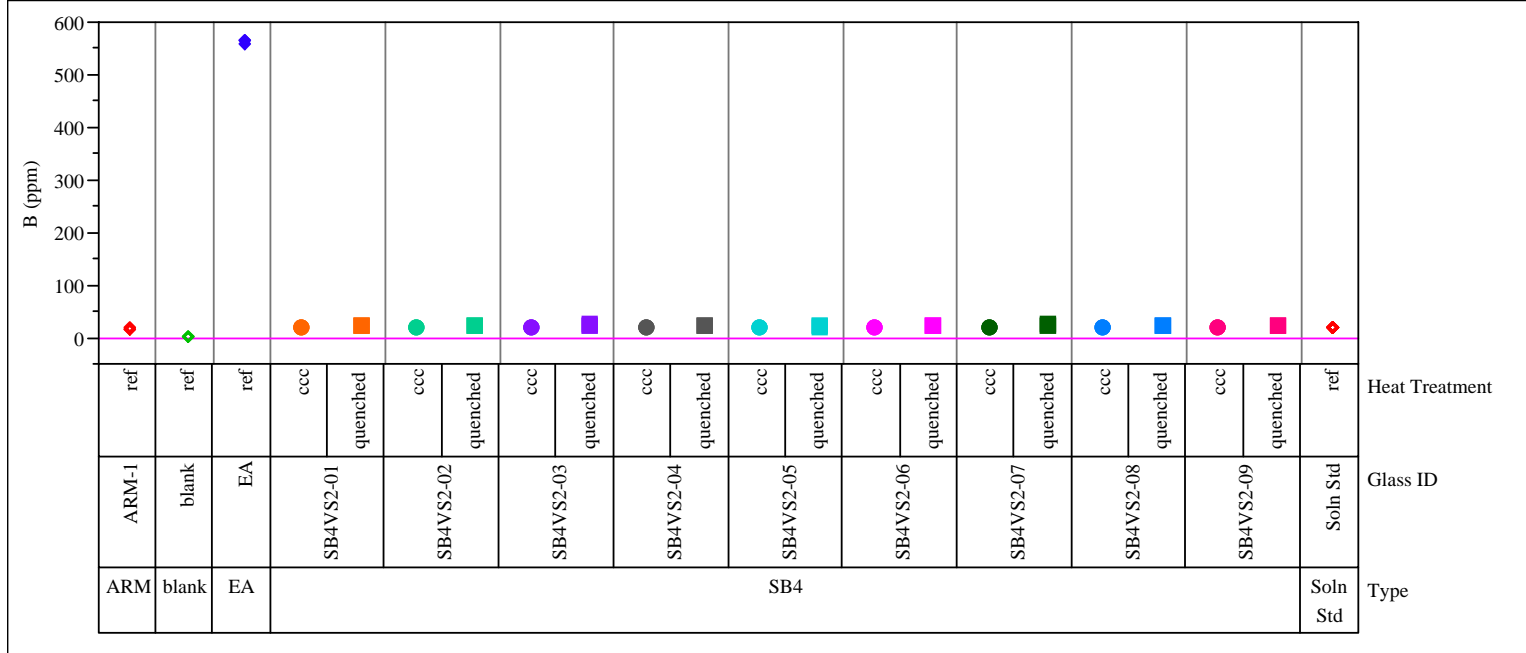

Variability Chart for Li (ppm)

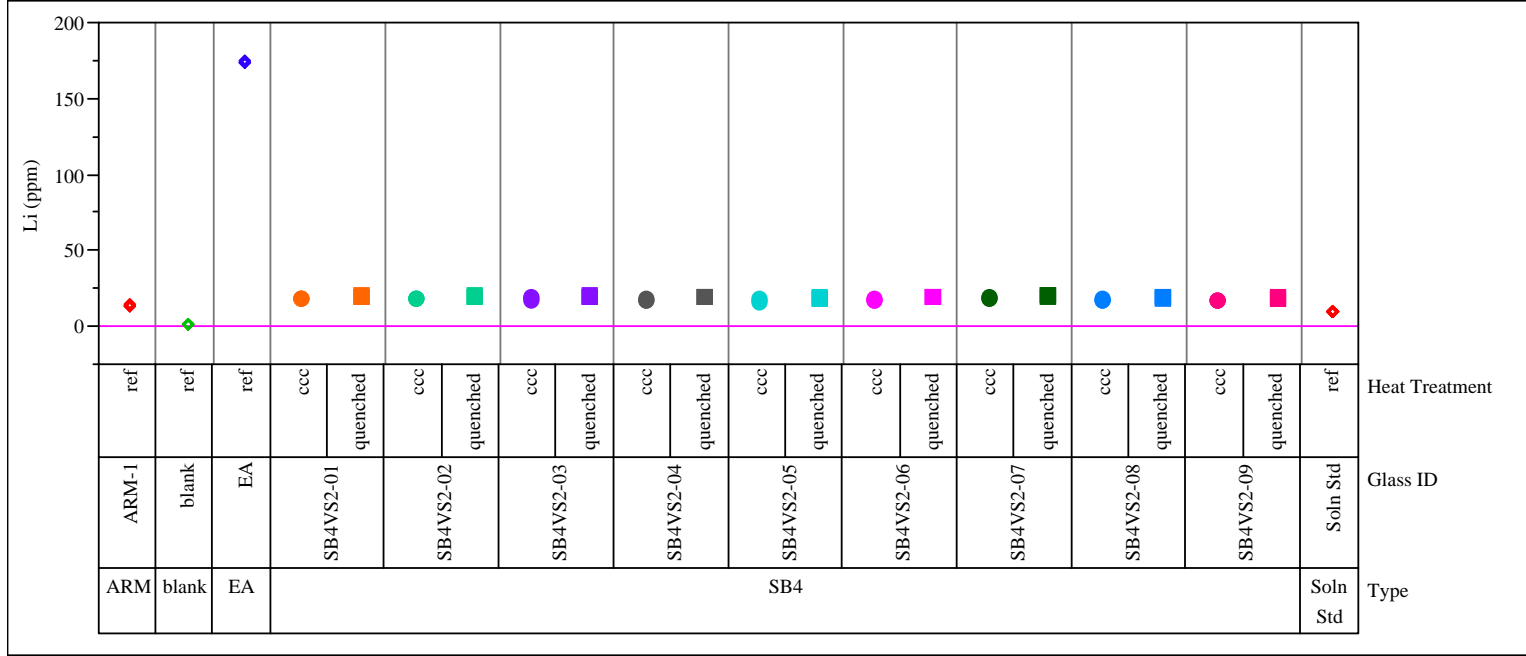


Figure B3. Laboratory PCT Measurements by Glass Identifier for Study Glasses and Standards

Variability Chart for Na (ppm)

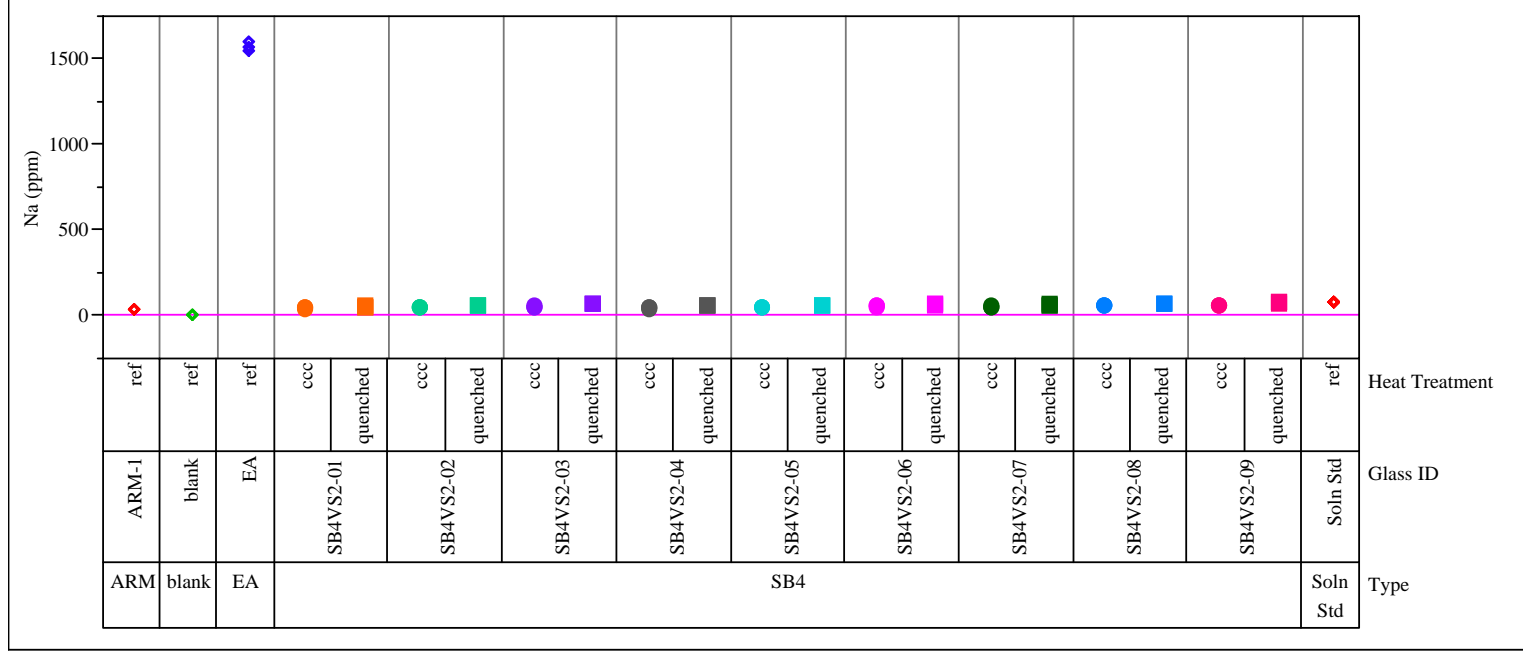

Variability Chart for Si (ppm)

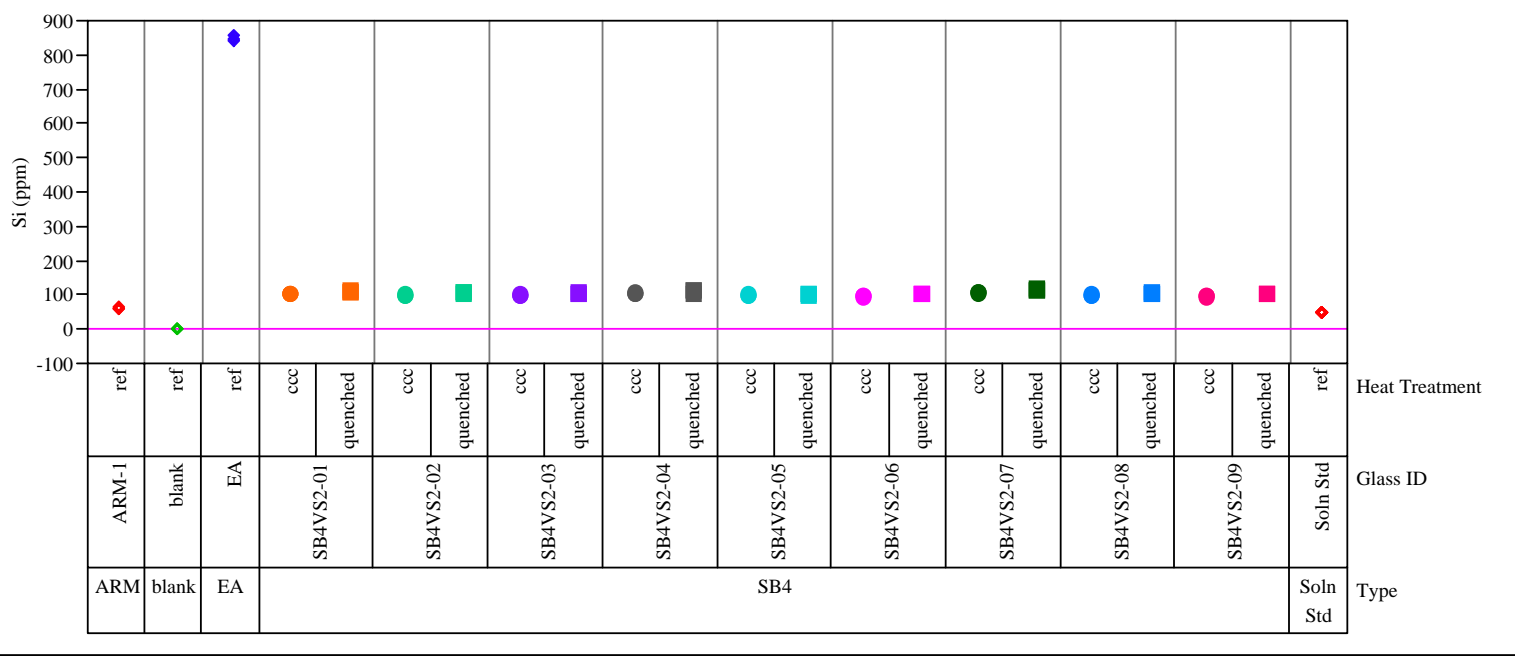


Figure B4. Laboratory PCT Measurements by Glass Identifier for Study Glasses

Variability Chart for B (ppm)

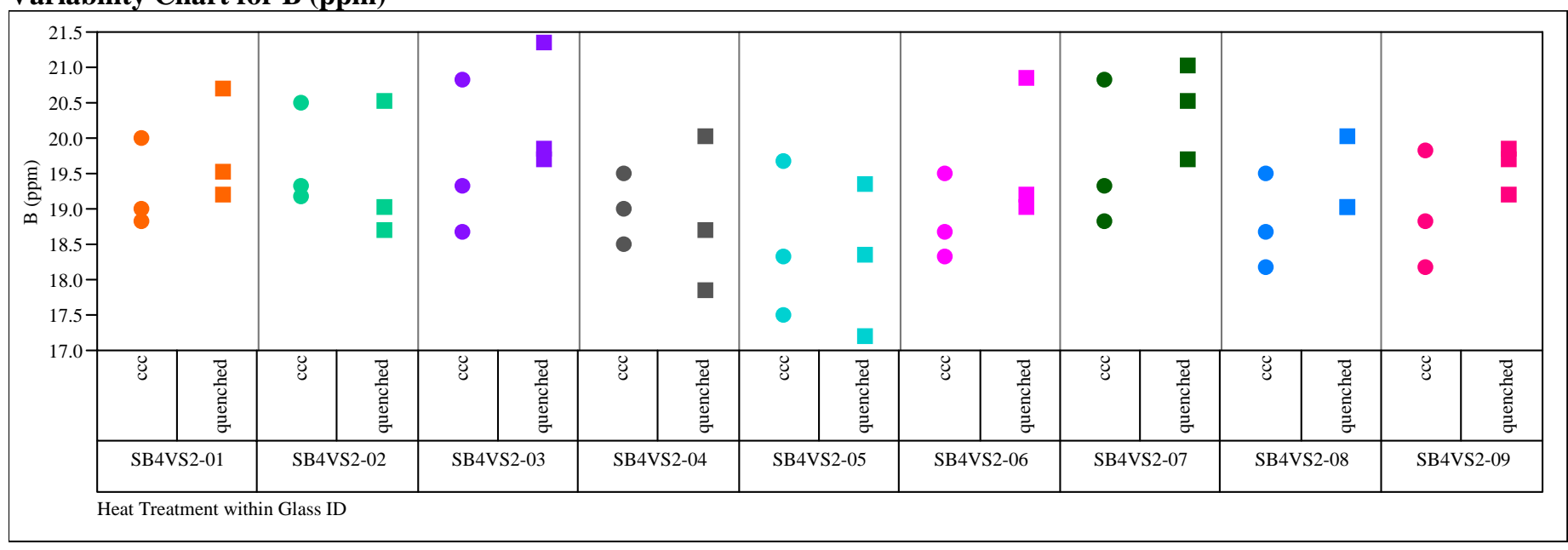

Variability Chart for Li (ppm)

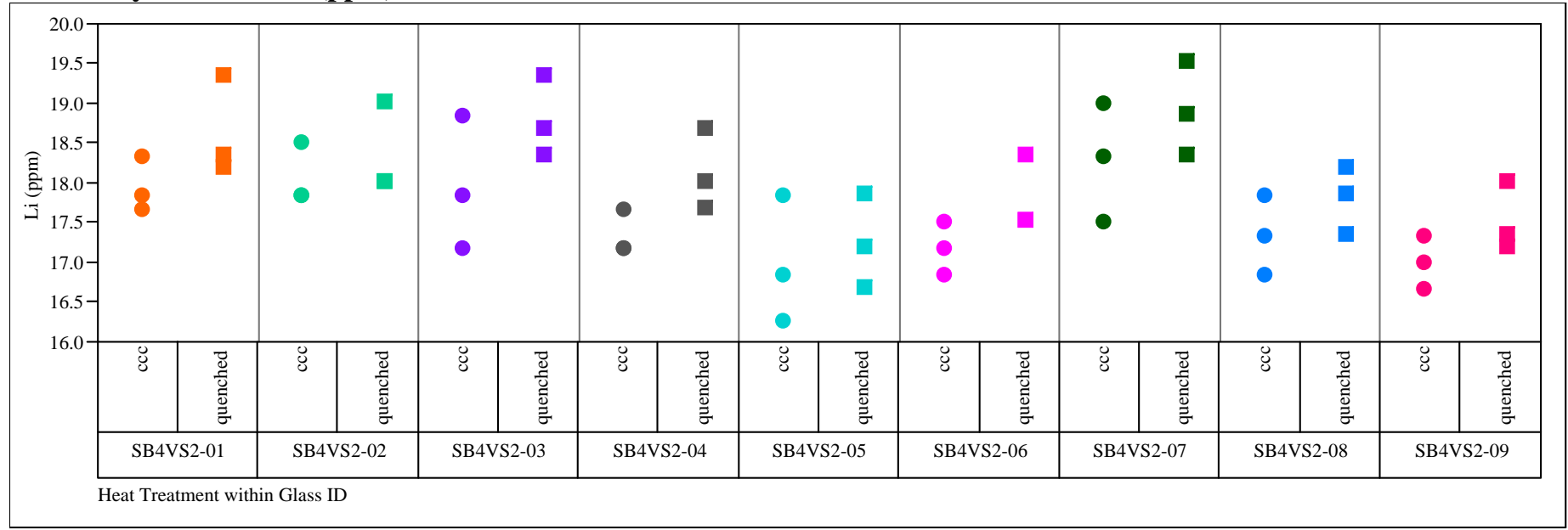


Figure B4. Laboratory PCT Measurements by Glass Identifier for Study Glasses

Variability Chart for Na (ppm)

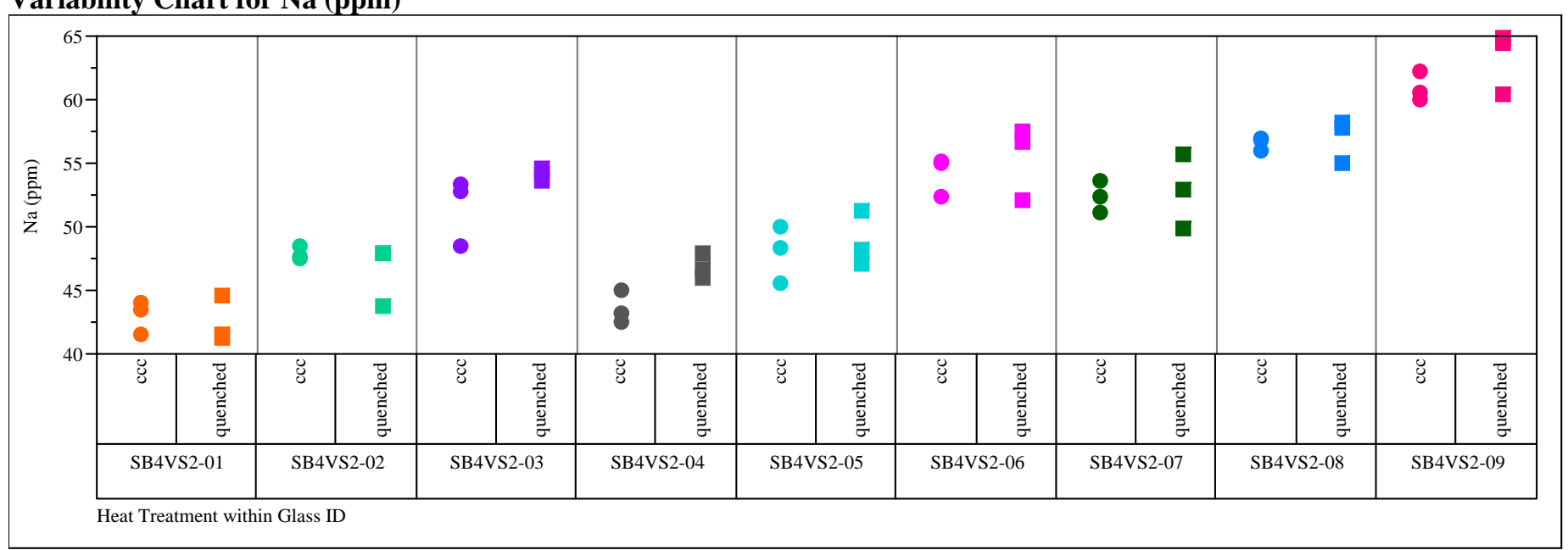

Variability Chart for Si (ppm)

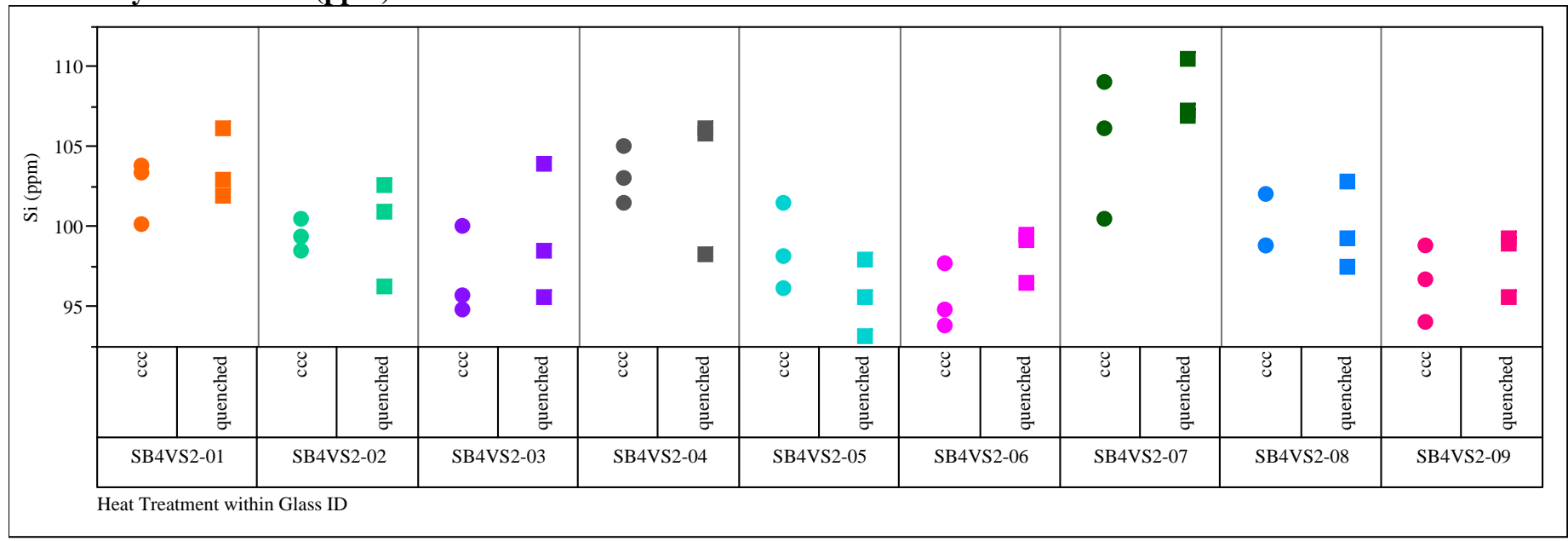


Figure B5. Correlations and Scatter Plots of Normalized PCTs Over All Compositional Views and Heat Treatments

\section{Correlations}

$\begin{array}{lrrrr} & \log \mathrm{NL}[\mathrm{B}(\mathrm{g} / \mathrm{L})] & \log \mathrm{NL}[\mathrm{Li}(\mathrm{g} / \mathrm{L})] & \log \mathrm{NL}[\mathrm{Na}(\mathrm{g} / \mathrm{L})] & \log \mathrm{NL}[\mathrm{Si}(\mathrm{g} / \mathrm{L})] \\ \log \mathrm{NL}[\mathrm{B}(\mathrm{g} / \mathrm{L})] & 1.0000 & 0.9984 & 0.9919 & 0.9882 \\ \log \mathrm{NL}[\mathrm{Li}(\mathrm{g} / \mathrm{L})] & 0.9984 & 1.0000 & 0.9891 & 0.9857 \\ \log \mathrm{NL}[\mathrm{Na}(\mathrm{g} / \mathrm{L})] & 0.9919 & 0.9891 & 1.0000 & 0.9749 \\ \log \mathrm{NL}[\mathrm{Si}(\mathrm{g} / \mathrm{L})] & 0.9882 & 0.9857 & 0.9749 & 1.0000\end{array}$

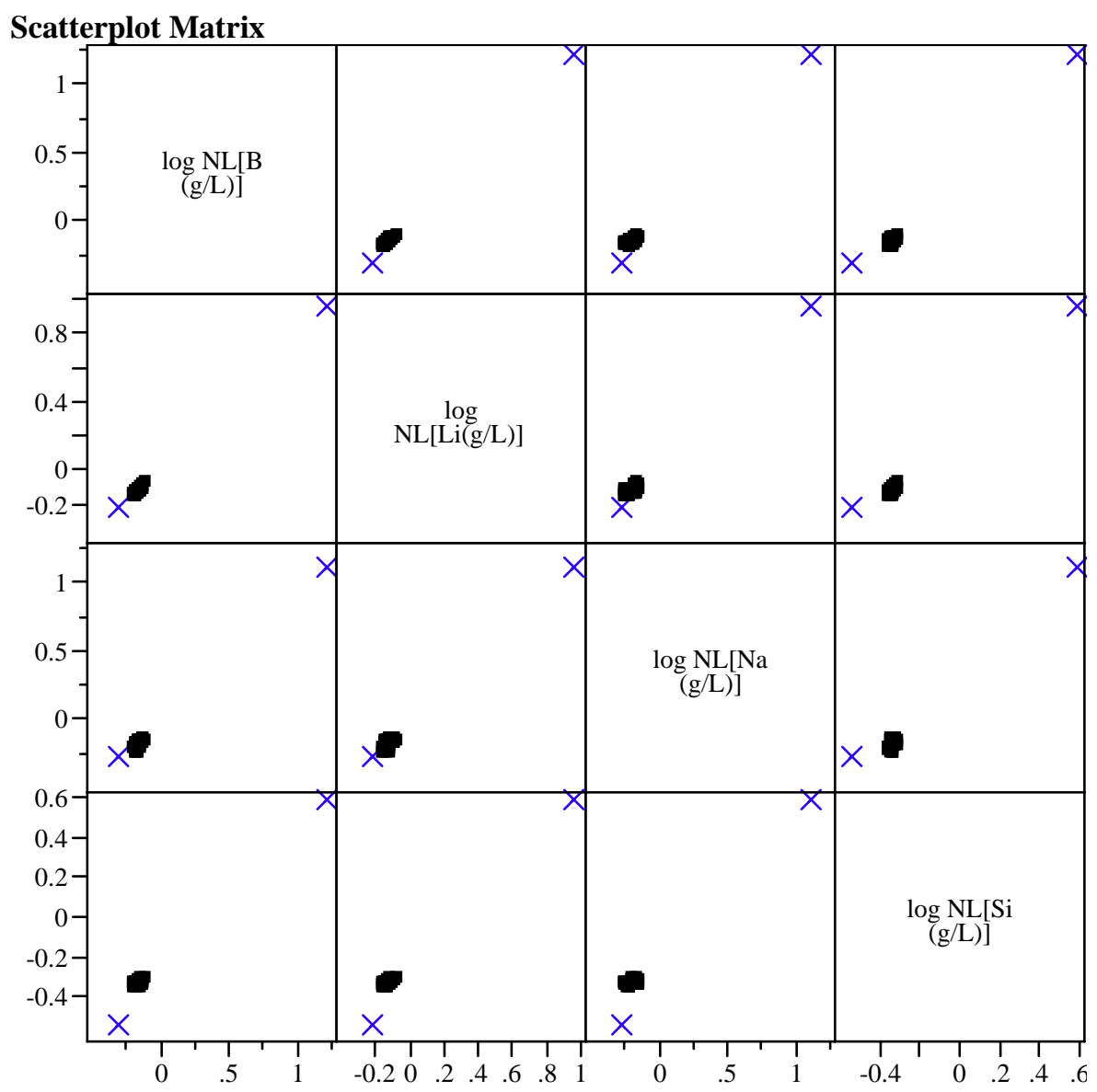

EA and ARM are both represented by " $X$.” 
Figure B6. Effects of Heat Treatment on PCT $\log (\mathrm{ppm})$-Response of Study Glasses

Oneway Analysis of $\log [\mathrm{B}$ ppm] By Heat Treatment Glass ID=SB4VS2-01

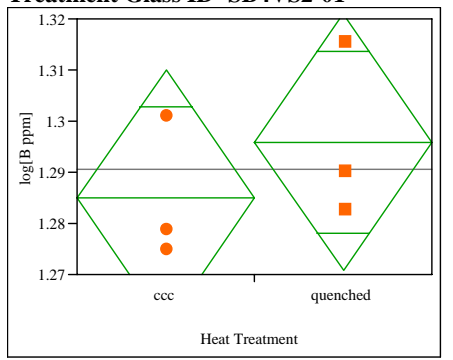

Difference $\quad 0.01105$ t Ratio 0.862038 Std Err Dif $\quad 0.01281$ DF 4 Upper CL Dif 0.04663 Prob $>|t| \quad 0.4373$ Lower CL Dif -0.02453 Prob $>t \quad 0.2186$ Confidence $\quad 0.95$ Prob $<\mathrm{t} \quad 0.7814$

Oneway Analysis of $\log [\mathrm{Li}$ ppm] By Heat Treatment Glass ID=SB4VS2-01

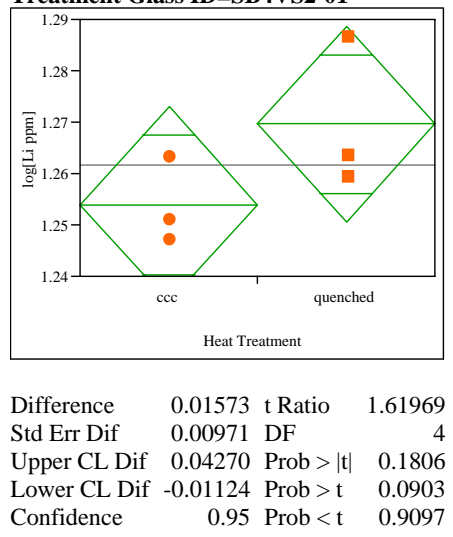

Oneway Analysis of log[Na ppm] By Heat Treatment Glass ID=SB4VS2-01

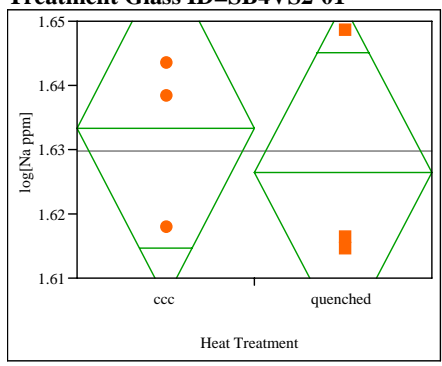

Difference $\quad-0.00693$ t Ratio $\quad-0.51462$ Std Err Dif 0.01346 DF

Upper CL Dif 0.03045 Prob $>|t| \quad 0.6339$

Lower CL Dif -0.04431 Prob $>\mathrm{t} \quad 0.6830$ Confidence
Oneway Analysis of $\log [\mathrm{Si} \mathrm{ppm}]$ By Heat Treatment Glass ID=SB4VS2-01

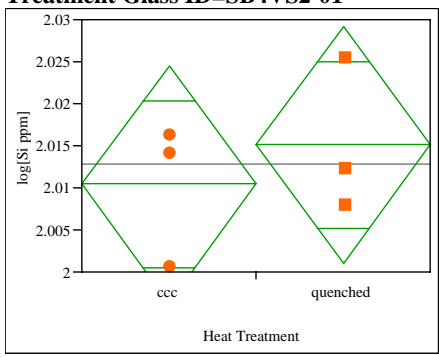

Difference $\quad 0.00468$ t Ratio 0.652095 Std Err Dif $\quad 0.00717$ DF

Upper CL Dif 0.02459 Prob $>|t| \quad 0.5499$ Lower CL Dif -0.01523 Prob $>$ t 0.2750

Confidence $\quad 0.95$ Prob $<\mathrm{t} \quad 0.7250$

Oneway Analysis of $\log [\mathrm{B}$ ppm] By Heat Treatment Glass ID=SB4VS2-02

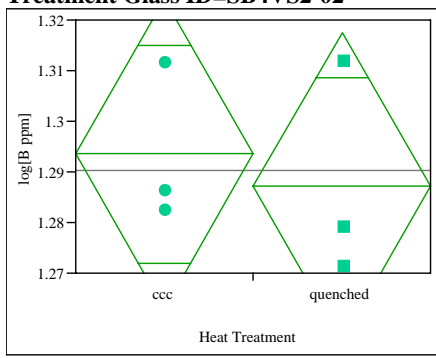

Difference $\quad-0.00634$ t Ratio $\quad-0.4096$ Std Err Dif $\quad 0.01549$ D

Upper CL Dif 0.03666 Prob $>|t| \quad 0.7031$ Lower CL Dif -0.04935 Prob $>$ t 0.6485

Confidence $\quad 0.95$ Prob $<\mathrm{t} \quad 0.3515$

Oneway Analysis of $\log [\mathrm{Li}$ ppm] By Heat Treatment Glass ID=SB4VS2-02

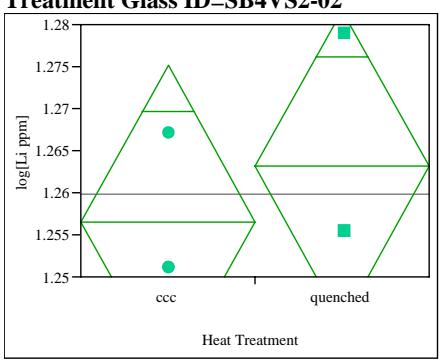

Difference $\quad 0.00655$ t Ratio 0.692808 Std Err Dif $\quad 0.00946$ DF

Upper CL Dif 0.03282 Prob $>|t| \quad 0.5266$ Lower CL Dif -0.01971 Prob $>\mathrm{t} \quad 0.2633$

Confidence $\quad 0.95$ Prob $<\mathrm{t} \quad 0.7367$
Oneway Analysis of $\log [\mathrm{Na} \mathrm{ppm}]$ By Heat Treatment Glass ID=SB4VS2-02

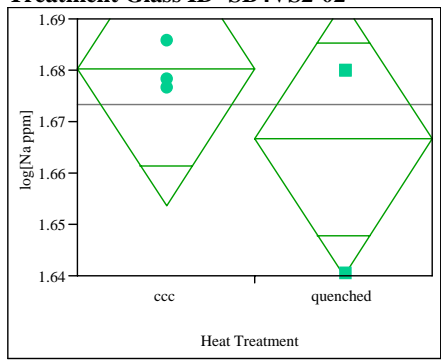

Difference $\quad-0.01368$ t Ratio $\quad-1.0143$ Std Err Dif $\quad 0.01349$ DF 4 Upper CL Dif 0.02377 Prob $>|t| \quad 0.3678$ Lower CL Dif -0.05112 Prob $>t \quad 0.8161$ Confidence $\quad 0.95$ Prob $<\mathrm{t} \quad 0.1839$

Oneway Analysis of $\log [\mathrm{Si}$ ppm] By Heat Treatment Glass ID=SB4VS2-02

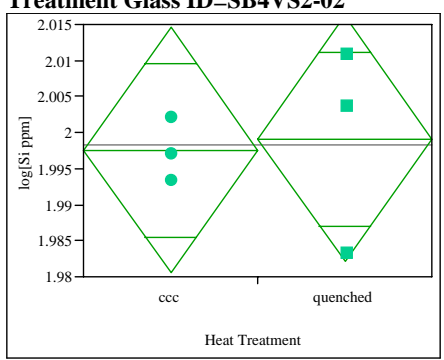

Difference $\quad 0.00155$ t Ratio 0.178733 Std Err Dif $\quad 0.00868$ DF

Upper CL Dif 0.02566 Prob $>|t| \quad 0.8668$

Lower CL Dif -0.02255 Prob >t 0.4334

$\begin{array}{lll}\text { Confidence } & 0.95 \text { Prob }<\mathrm{t} \quad 0.5666\end{array}$

Oneway Analysis of $\log [\mathrm{B}$ ppm] By Heat Treatment Glass ID=SB4VS2-03

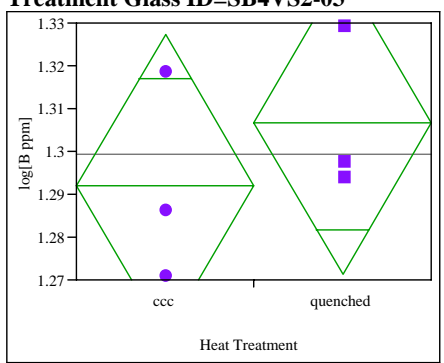

Difference $\quad 0.01468$ t Ratio 0.816358 Std Err Dif $\quad 0.01799$ DF

Upper CL Dif 0.06463 Prob $>|t| \quad 0.4601$ Lower CL Dif -0.03526 Prob $>t \quad 0.2301$ $\begin{array}{lrll}\text { Confidence } & 0.95 \text { Prob }<\mathrm{t} & 0.7699\end{array}$ 
Figure B6. Effects of Heat Treatment on PCT $\log (\mathrm{ppm})$-Response of Study Glasses

Oneway Analysis of $\log [\mathrm{Li} \mathrm{ppm}]$ By Heat Treatment Glass ID=SB4VS2-03

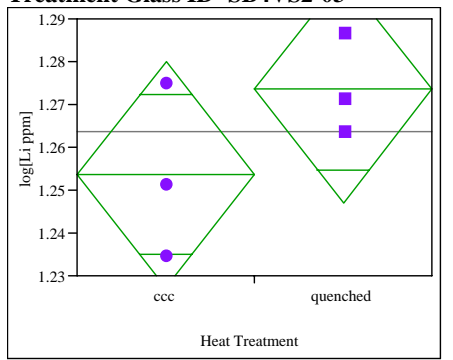

Difference $\quad 0.01992$ t Ratio 1.475878 Std Err Dif $\quad 0.01350$ DF Upper CL Dif 0.05740 Prob $>|t| \quad 0.2140$ Lower CL Dif -0.01756 Prob $>\mathrm{t} \quad 0.1070$ Confidence $\quad 0.95$ Prob $<\mathrm{t} \quad 0.8930$

Oneway Analysis of log[Na ppm] By Heat Treatment Glass ID=SB4VS2-03

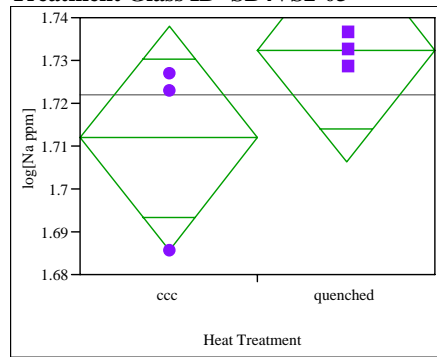

Difference $\quad 0.02050$ t Ratio $\quad 1.538054$ Std Err Dif $\quad 0.01333$ DF

Upper CL Dif 0.05750 Prob $>|t| \quad 0.1989$ Lower CL Dif -0.01650 Prob $>t \quad 0.0994$ $\begin{array}{lll}\text { Confidence } & 0.95 \text { Prob }<\mathrm{t} \quad 0.9006\end{array}$

Oneway Analysis of log[Si ppm] By Heat Treatment Glass ID=SB4VS2-03

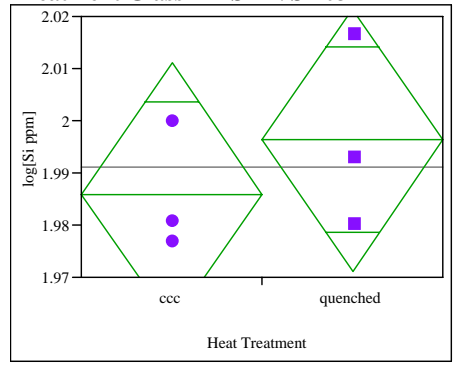

Difference $\quad 0.01044$ t Ratio 0.814741 Std Err Dif $\quad 0.01281$ DF Upper CL Dif 0.04602 Prob $>|t| \quad 0.4609$ Lower CL Dif -0.02514 Prob $>t \quad 0.2305$ Confidence $\quad 0.95$ Prob $<\mathrm{t} \quad 0.7695$
Oneway Analysis of $\log [B$ ppm] By Heat Treatment Glass ID=SB4VS2-04

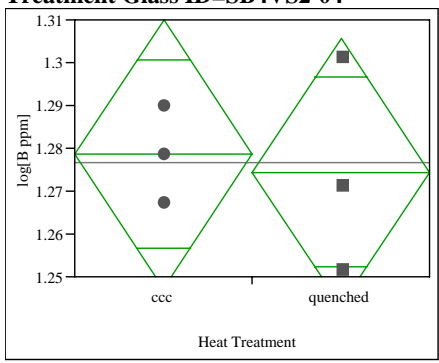

Difference $\quad-0.00421$ t Ratio $\quad-0.26466$ Std Err Dif $\quad 0.01591$ DF

Upper CL Dif 0.03996 Prob $>|t| \quad 0.8043$

Lower CL Dif -0.04838 Prob $>t \quad 0.5978$

Confidence $\quad 0.95$ Prob $<\mathrm{t} \quad 0.4022$

Oneway Analysis of $\log [\mathrm{Li}$ ppm] By Heat Treatment Glass ID=SB4VS2-04

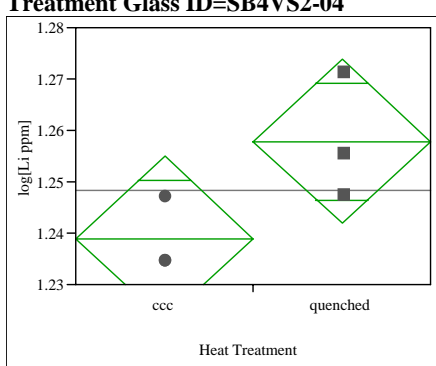

Difference $\quad 0.01899$ t Ratio 2.32754

Upper CL Dif 0.04164 Prob $>|t| 0.0805$

Lower CL Dif -0.00366 Prob $>t \quad 0.0402$

$\begin{array}{lll}\text { Confidence } & 0.95 \text { Prob }<\mathrm{t} & 0.9598\end{array}$

Oneway Analysis of log[Na ppm] By Heat Treatment Glass ID=SB4VS2-04

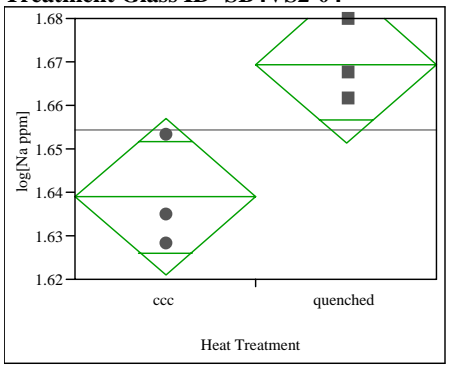

Difference 0.030538 t Ratio 3.320655 Std Err Dif $\quad 0.009196$ DF

Upper CL Dif 0.056072 Prob $>|t| \quad 0.0294$ Lower CL Dif 0.005005 Prob $>t \quad 0.0147$

Confidence $\quad 0.95$ Prob $<\mathrm{t} \quad 0.9853$
Oneway Analysis of $\log [\mathrm{Si} \mathrm{ppm}]$ By Heat Treatment Glass ID=SB4VS2-04

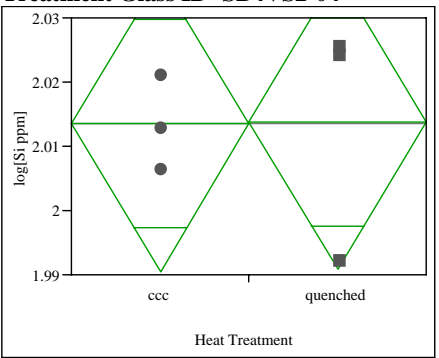

Difference $\quad 0.00024$ t Ratio 0.020385 Std Err Dif $\quad 0.01170$ DF

Upper CL Dif 0.03272 Prob $>|t| \quad 0.9847$

Lower CL Dif -0.03224 Prob $>t \quad 0.4924$

Confidence $\quad 0.95$ Prob $<\mathrm{t} \quad 0.5076$

Oneway Analysis of $\log [\mathrm{B}$ ppm] By Heat Treatment Glass ID=SB4VS2-05

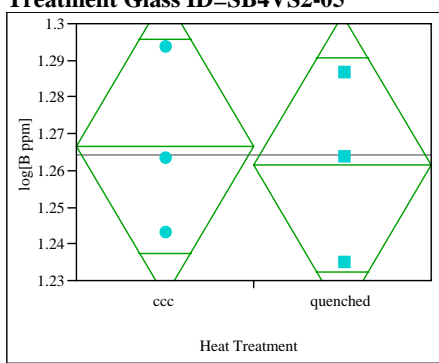

Difference $\quad-0.00526$ t Ratio $\quad-0.2507$ Std Err Dif $\quad 0.02098$ DF 4 Upper CL Dif 0.05298 Prob $>|\mathrm{t}| \quad 0.8144$ Lower CL Dif -0.06350 Prob $>t \quad 0.5928$ $\begin{array}{lll}\text { Confidence } & 0.95 \text { Prob }<\mathrm{t} & 0.4072\end{array}$

Oneway Analysis of log[Li ppm] By Heat Treatment Glass ID=SB4VS2-05

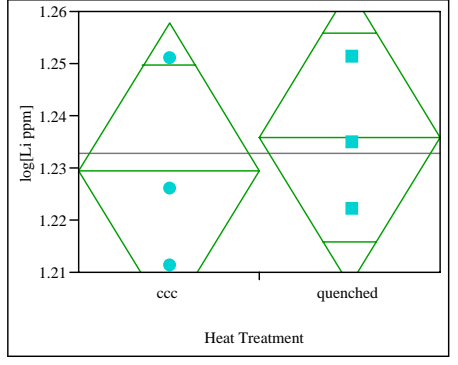

Difference $\quad 0.00636$ t Ratio 0.440546 Std Err Dif $\quad 0.01443$ DF

Upper CL Dif 0.04641 Prob $>|t| \quad 0.6823$ Lower CL Dif -0.03370 Prob $>\mathrm{t} \quad 0.3412$ $\begin{array}{lll}\text { Confidence } & 0.95 \text { Prob }<\mathrm{t} \quad 0.6588\end{array}$ 
Figure B6. Effects of Heat Treatment on PCT $\log (\mathrm{ppm})$-Response of Study Glasses

Oneway Analysis of $\log [\mathrm{Na} \mathrm{ppm}]$ By Heat Treatment Glass ID=SB4VS2-05

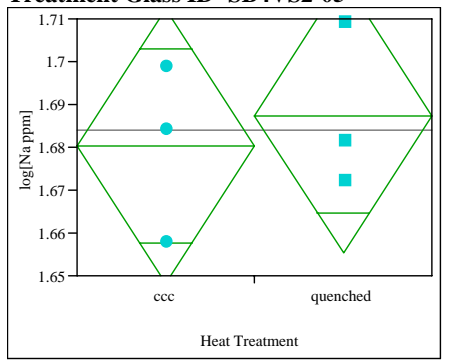

Difference $\quad 0.00703$ t Ratio 0.4308 Std Err Dif $\quad 0.01632$ D

Upper CL Dif 0.05236 Prob $>|t| 0.6888$

Lower CL Dif -0.03829 Prob $>t \quad 0.3444$

Confidence $\quad 0.95$ Prob $<\mathrm{t} \quad 0.6556$

Oneway Analysis of $\log [\mathrm{Si}$ ppm] By Heat Treatment Glass ID=SB4VS2-05

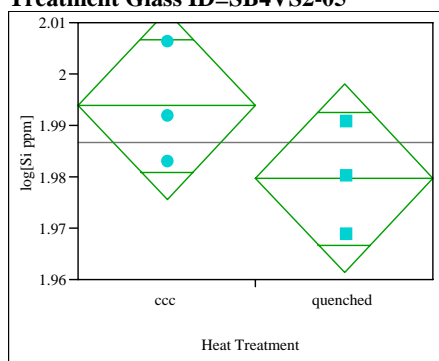

Difference $\quad-0.01416$ t Ratio $\quad-1.51791$ Std Err Dif $\quad 0.00933$

Upper CL Dif 0.01174 Prob $>|\mathrm{t}| \quad 0.2036$

Lower CL Dif -0.04006 Prob $>t \quad 0.8982$

$\begin{array}{lll}\text { Confidence } & 0.95 \text { Prob }<\mathrm{t} \quad 0.1018\end{array}$

Oneway Analysis of $\log [\mathrm{B}$ ppm $]$ By Heat Treatment Glass ID=SB4VS2-06

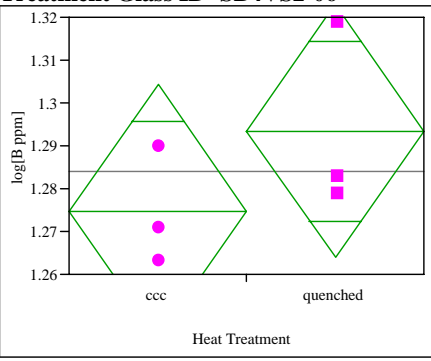

Difference $\quad 0.01857$ t Ratio 1.235848 Std Err Dif $\quad 0.01503$ DF

Upper CL Dif 0.06030 Prob $>|t| \quad 0.2841$

Lower CL Dif -0.02315 Prob $>t \quad 0.1421$

Confidence $\quad 0.95$ Prob $<\mathrm{t} \quad 0.8579$
Oneway Analysis of $\log [\mathrm{Li} \mathrm{ppm}]$ By Heat Treatment Glass ID=SB4VS2-06

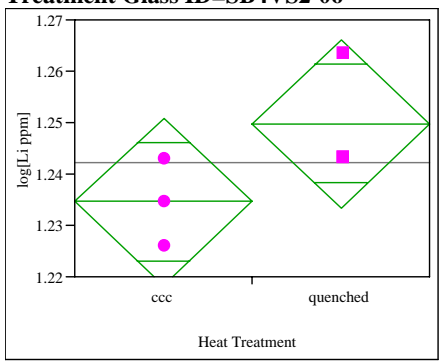

Difference $\quad 0.01514$ t Ratio 1.821929 Std Err Dif $\quad 0.00831$ DF

Upper CL Dif 0.03821 Prob $>|t| \quad 0.1426$

Lower CL Dif -0.00793 Prob $>t \quad 0.0713$

Confidence $\quad 0.95$ Prob $<\mathrm{t} \quad 0.9287$

Oneway Analysis of log[Na ppm] By Heat Treatment Glass ID=SB4VS2-06

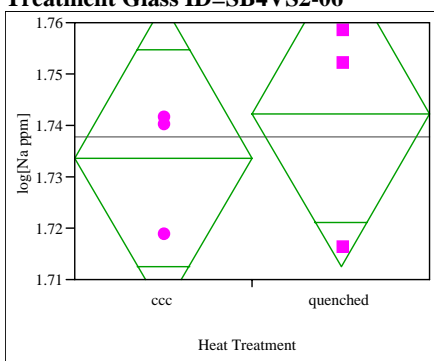

Difference $\quad 0.00855$ t Ratio 0.564277 Std Err Dif $\quad 0.01515$ DF

Upper CL Dif 0.05060 Prob > t $\mid 0.6027$

Lower CL Dif -0.03351 Prob $>t \quad 0.3014$

$\begin{array}{lll}\text { Confidence } & 0.95 \text { Prob }<\mathrm{t} & 0.6986\end{array}$

Oneway Analysis of $\log [\mathrm{Si}$ ppm] By Heat Treatment Glass ID $=$ SB4VS2-06

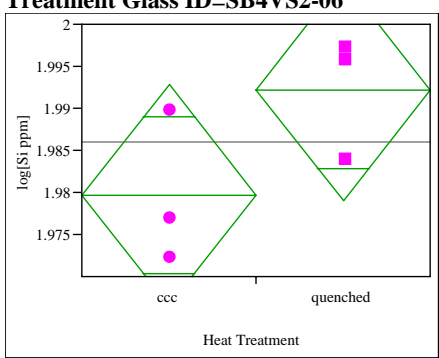

Difference $\quad 0.01248$ t Ratio 1.863764 Std Err Dif $\quad 0.00670$ DF

Upper CL Dif 0.03107 Prob $>|\mathrm{t}| \quad 0.1358$

Lower CL Dif -0.00611 Prob $>t \quad 0.0679$

$\begin{array}{lll}\text { Confidence } & 0.95 \text { Prob }<\mathrm{t} & 0.9321\end{array}$
Oneway Analysis of $\log [\mathrm{B}$ ppm] By Heat

Treatment Glass ID=SB4VS2-07

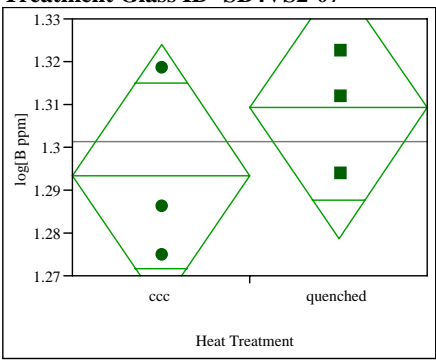

Difference $\quad 0.01590$ t Ratio 1.023061 Std Err Dif $\quad 0.01555$ DF

Upper CL Dif 0.05906 Prob $>|t| \quad 0.3641$

Lower CL Dif -0.02726 Prob $>t \quad 0.1821$

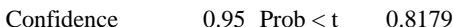

Oneway Analysis of $\log [\mathrm{Li}$ ppm] By Heat Treatment Glass ID=SB4VS2-07

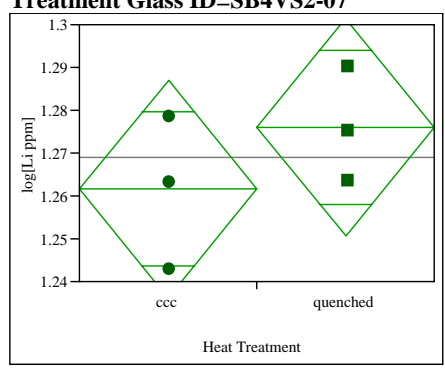

Difference $\quad 0.01439$ t Ratio 1.113337 Std Err Dif $\quad 0.01293$ DF

Upper CL Dif 0.05028 Prob $>|t| \quad 0.3280$

Lower CL Dif -0.02150 Prob $>t \quad 0.1640$

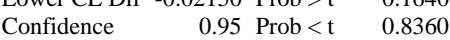

Oneway Analysis of log[Na ppm] By Heat Treatment Glass ID $=$ SB4VS2-07

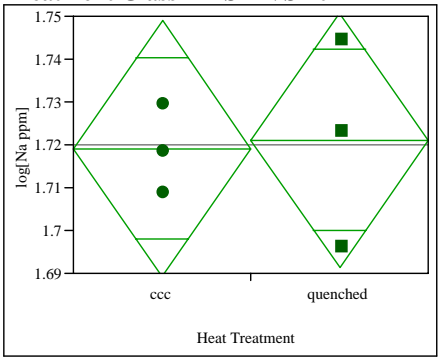

Difference $\quad 0.00193$ t Ratio 0.127262 Std Err Dif 0.01518 DF

Upper CL Dif 0.04408 Prob $>|t| \quad 0.9049$

Lower CL Dif - 0.04022 Prob $>t \quad 0.4524$

Confidence $\quad 0.95$ Prob $<\mathrm{t} \quad 0.5476$ 
Figure B6. Effects of Heat Treatment on PCT $\log (\mathrm{ppm})$-Response of Study Glasses

Oneway Analysis of $\log [\mathrm{Si}$ ppm] By Heat Treatment Glass ID $=$ SB4VS2-07

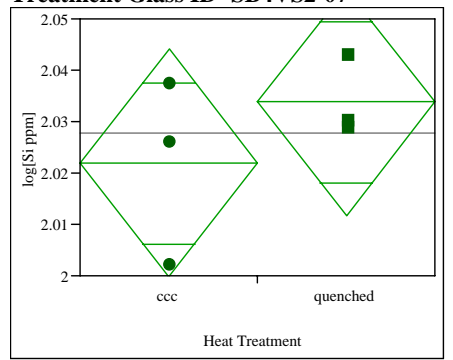

Difference $\quad 0.01196$ t Ratio 1.058549 Std Err Dif $\quad 0.01130$ DF Upper CL Dif 0.04334 Prob $>|t| \quad 0.3495$ Lower CL Dif -0.01942 Prob $>\mathrm{t} \quad 0.1747$ Confidence $\quad 0.95$ Prob $<\mathrm{t} \quad 0.8253$

Oneway Analysis of $\log [\mathrm{B}$ ppm] By Heat Treatment Glass ID=SB4VS2-08

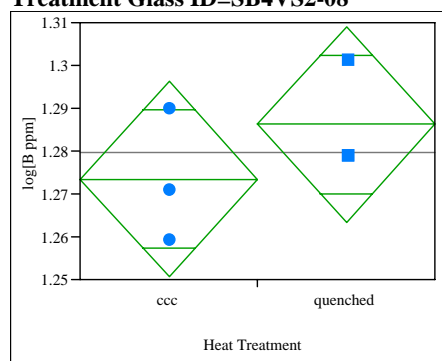

Difference $\quad 0.01272$ t Ratio 1.093109 Std Err Dif $\quad 0.01164$ DF

Upper CL Dif 0.04503 Prob $>|t| \quad 0.3358$

Lower CL Dif -0.01959 Prob $>t \quad 0.1679$

$\begin{array}{lrl}\text { Confidence } & 0.95 \text { Prob }<\mathrm{t} & 0.8321\end{array}$

Oneway Analysis of $\log [\mathrm{Li}$ ppm] By Heat Treatment Glass ID=SB4VS2-08

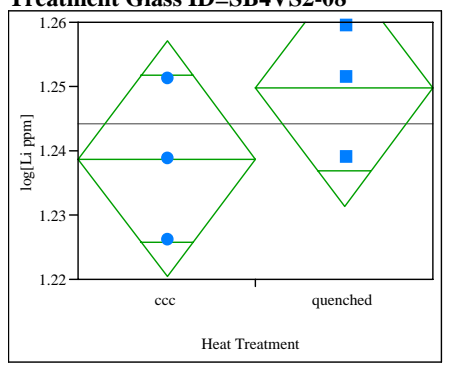

Difference $\quad 0.01104$ t Ratio 1.179564 Std Err Dif $\quad 0.00936$ DF

Upper CL Dif 0.03701 Prob $>|t| \quad 0.3035$

Lower CL Dif -0.01494 Prob $>t \quad 0.1518$

$\begin{array}{lll}\text { Confidence } & 0.95 \text { Prob }<\mathrm{t} \quad 0.8482\end{array}$
Oneway Analysis of $\log [\mathrm{Na} \mathrm{ppm}]$ By Heat Treatment Glass ID=SB4VS2-08

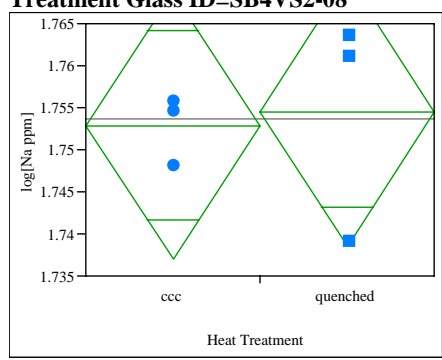

Difference $\quad 0.00158$ t Ratio $\quad 0.194675$ Std Err Dif $\quad 0.00810$ DF

Upper CL Dif 0.02407 Prob $>|t| \quad 0.8551$ Lower CL Dif -0.02092 Prob $>$ t 0.4276

Confidence $\quad 0.95$ Prob $<\mathrm{t} \quad 0.5724$

Oneway Analysis of log[Si ppm] By Heat Treatment Glass ID=SB4VS2-08

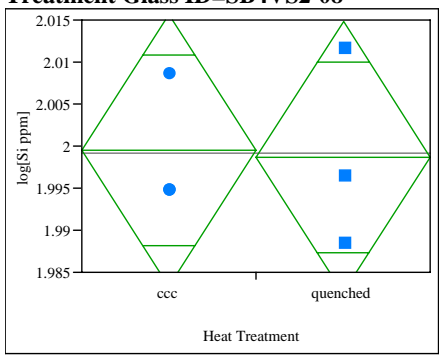

Difference $\quad-0.00078$ t Ratio $\quad-0.09577$ Std Err Dif $\quad 0.00818$ DF

Upper CL Dif 0.02193 Prob $>|t| \quad 0.9283$

Lower CL Dif -0.02350 Prob $>\mathrm{t} \quad 0.5358$

Confidence $\quad 0.95$ Prob $<\mathrm{t} \quad 0.4642$

Oneway Analysis of $\log [\mathrm{B}$ ppm] By Heat Treatment Glass ID=SB4VS2-09

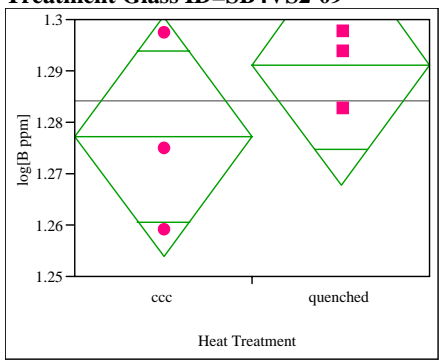

Difference $\quad 0.01402$ t Ratio 1.17557 Std Err Dif $\quad 0.01193$ DF Upper CL Dif 0.04715 Prob $>|t| \quad 0.3050$ Lower CL Dif -0.01910 Prob $>t \quad 0.1525$ Confidence $\quad 0.95$ Prob $<\mathrm{t} \quad 0.8475$
Oneway Analysis of $\log [\mathrm{Li} \mathrm{ppm}]$ By Heat Treatment Glass ID=SB4VS2-09

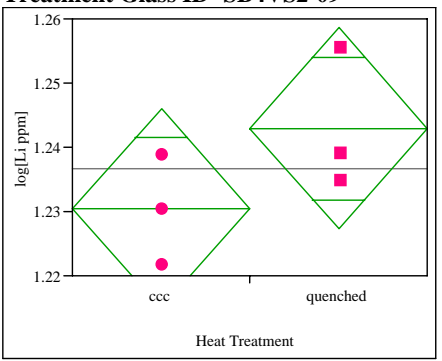

Difference $\quad 0.01255$ t Ratio 1.573801 Std Err Dif $\quad 0.00798$ DF

Upper CL Dif 0.03470 Prob $>|t| \quad 0.1906$

Lower CL Dif -0.00959 Prob $>t \quad 0.0953$

$\begin{array}{lll}\text { Confidence } & 0.95 \text { Prob }<\mathrm{t} \quad 0.9047\end{array}$

Oneway Analysis of $\log [\mathrm{Na} \mathrm{ppm}]$ By Heat Treatment Glass ID=SB4VS2-09

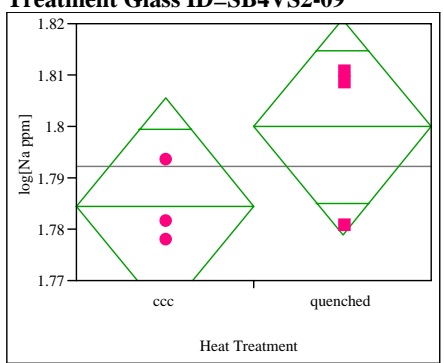

Difference $\quad 0.01540$ t Ratio 1.433141 Std Err Dif 0.01075 DF 4 Upper CL Dif 0.04524 Prob $>|t| \quad 0.2251$ Lower CL Dif -0.01444 Prob $>t \quad 0.1126$ $\begin{array}{lll}\text { Confidence } & 0.95 \text { Prob }<\mathrm{t} & 0.8874\end{array}$

Oneway Analysis of $\log [\mathrm{Si}$ ppm] By Heat Treatment Glass ID=SB4VS2-09

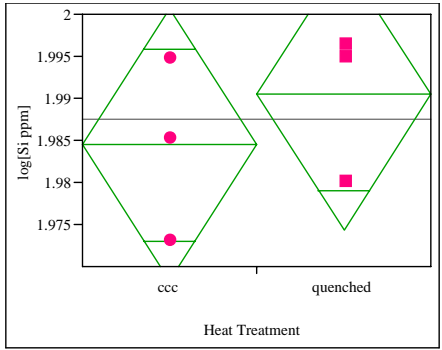

Difference $\quad 0.00599$ t Ratio 0.731473 Std Err Dif 0.00819 DF 4 Upper CL Dif 0.02872 Prob $>|t| \quad 0.5050$ Lower CL Dif -0.01674 Prob $>\mathrm{t} \quad 0.2525$ $\begin{array}{lll}\text { Confidence } & 0.95 \text { Prob }<\mathrm{t} & 0.7475\end{array}$ 
Figure B7. Effects of Heat Treatment for Study Glasses by Compositional View

Comp View=measured

Variability Chart for log NL[B (g/L)]

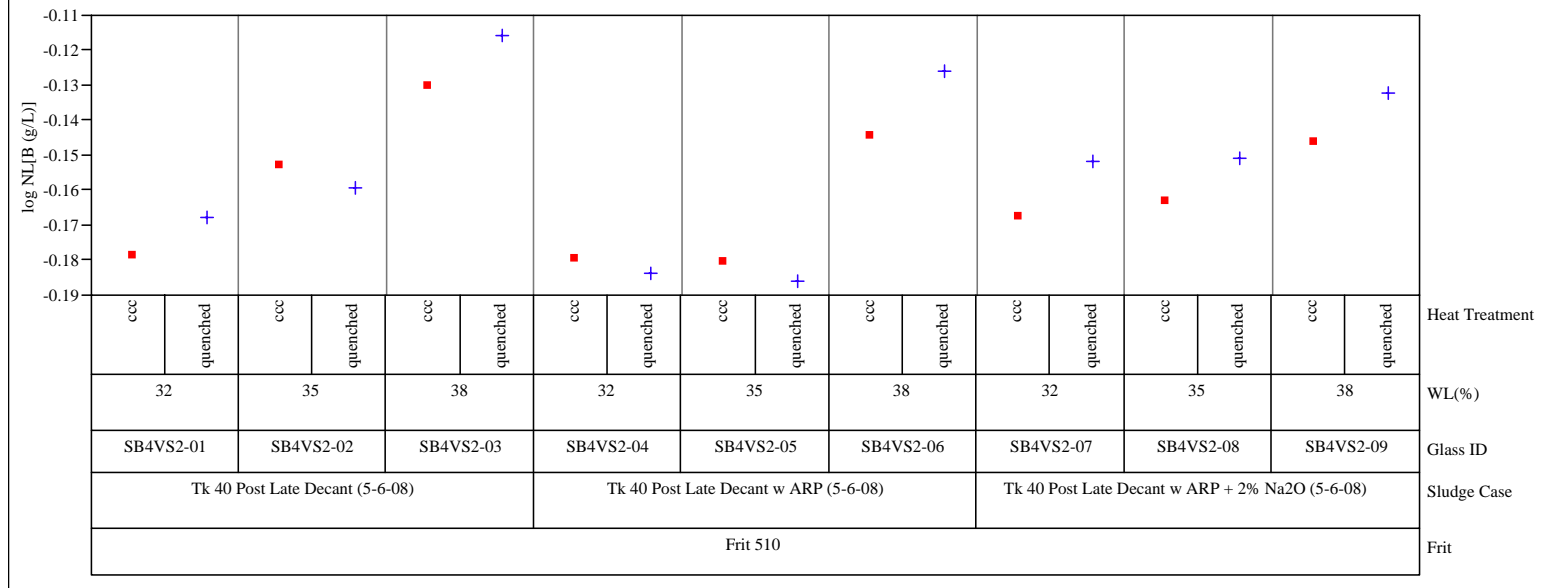

\section{Comp View=measured}

Variability Chart for log NL[Li(g/L)]

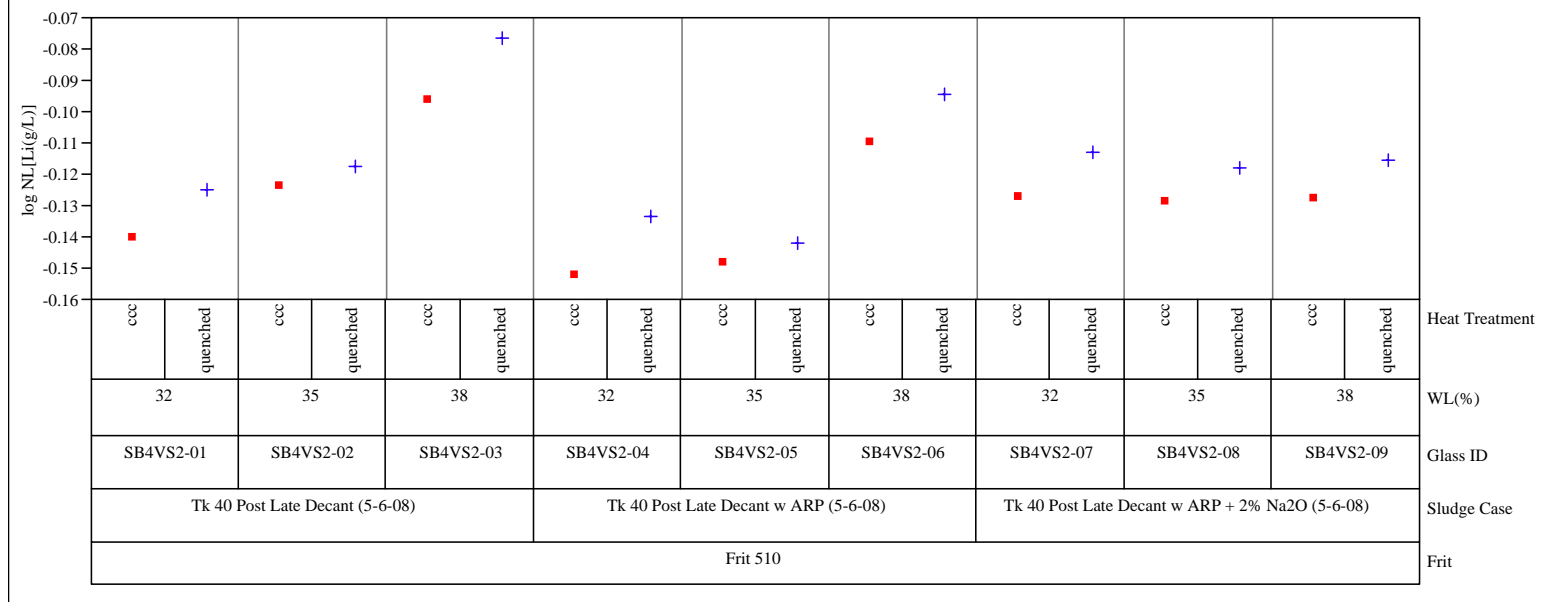

\section{Comp View=measured}

Variability Chart for $\log$ NL[Na (g/L)]

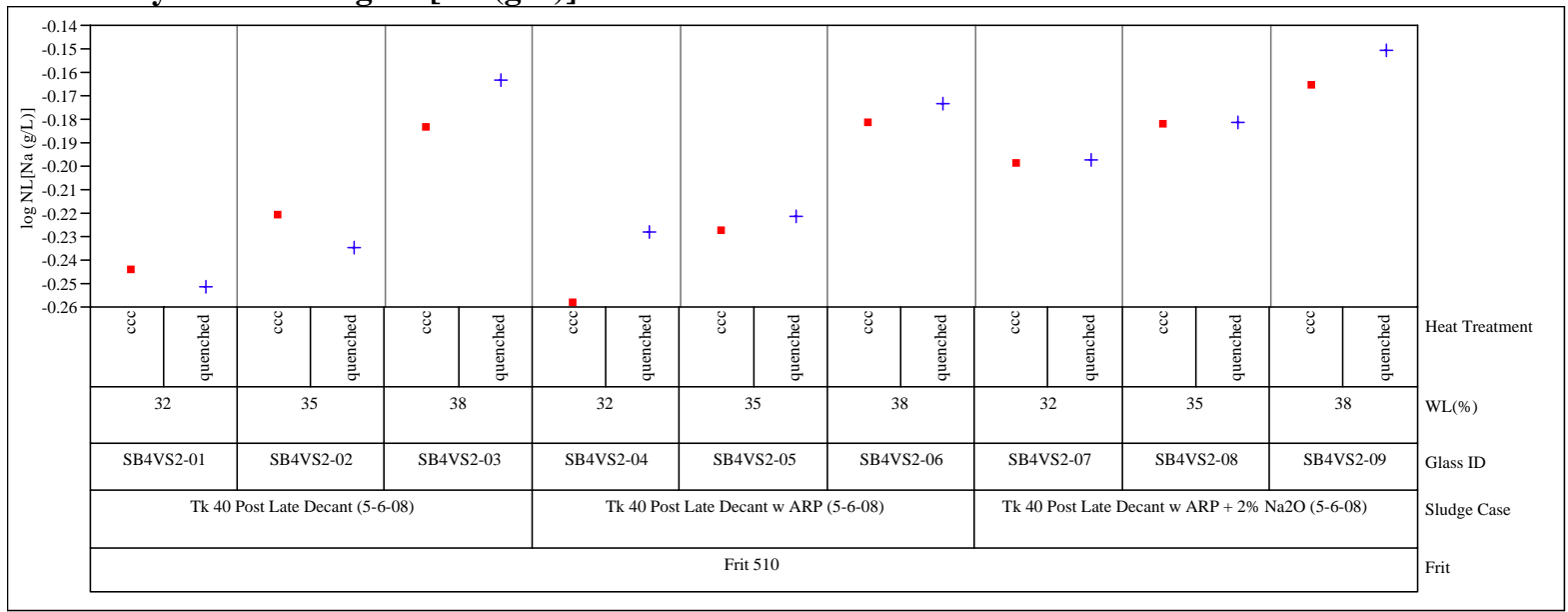


Figure B7. Effects of Heat Treatment for Study Glasses by Compositional View

\section{Comp View=measured}

Variability Chart for $\log$ NL[Si (g/L)]

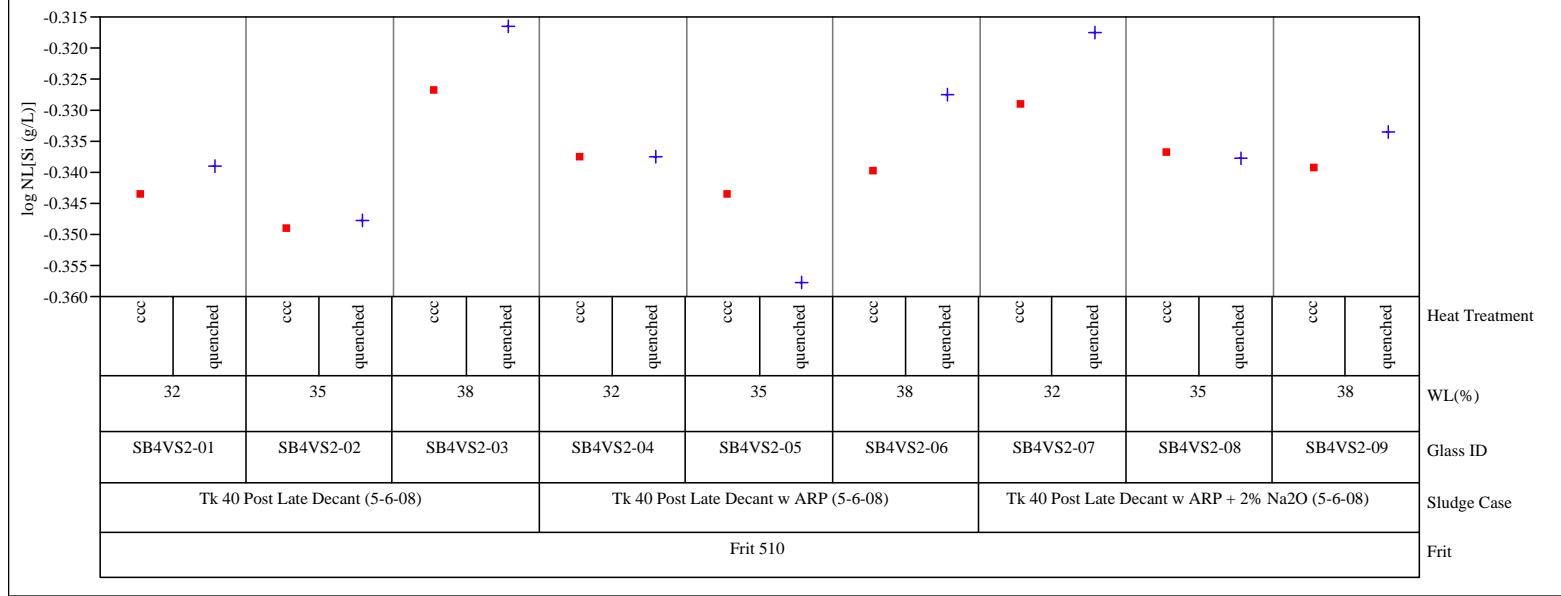

Comp View=measured bc

Variability Chart for log NL[B (g/L)]

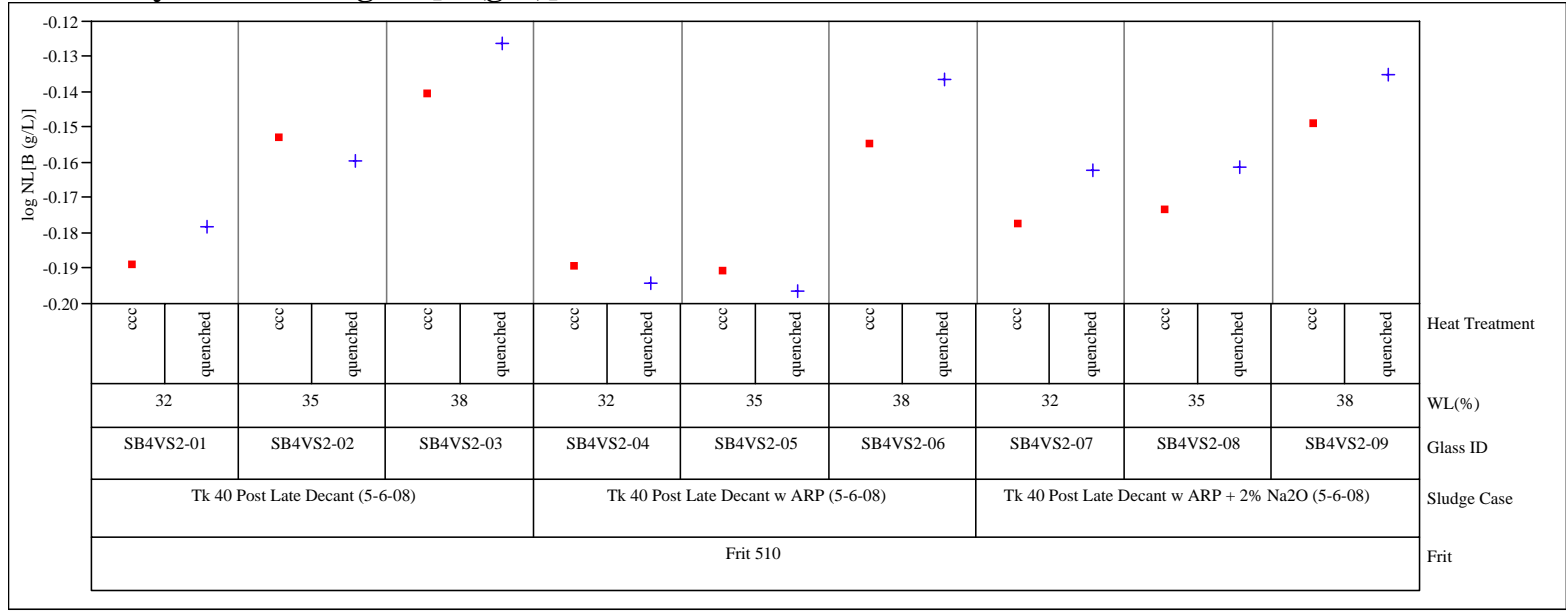

Comp View=measured bc

Variability Chart for log NL[Li(g/L)]

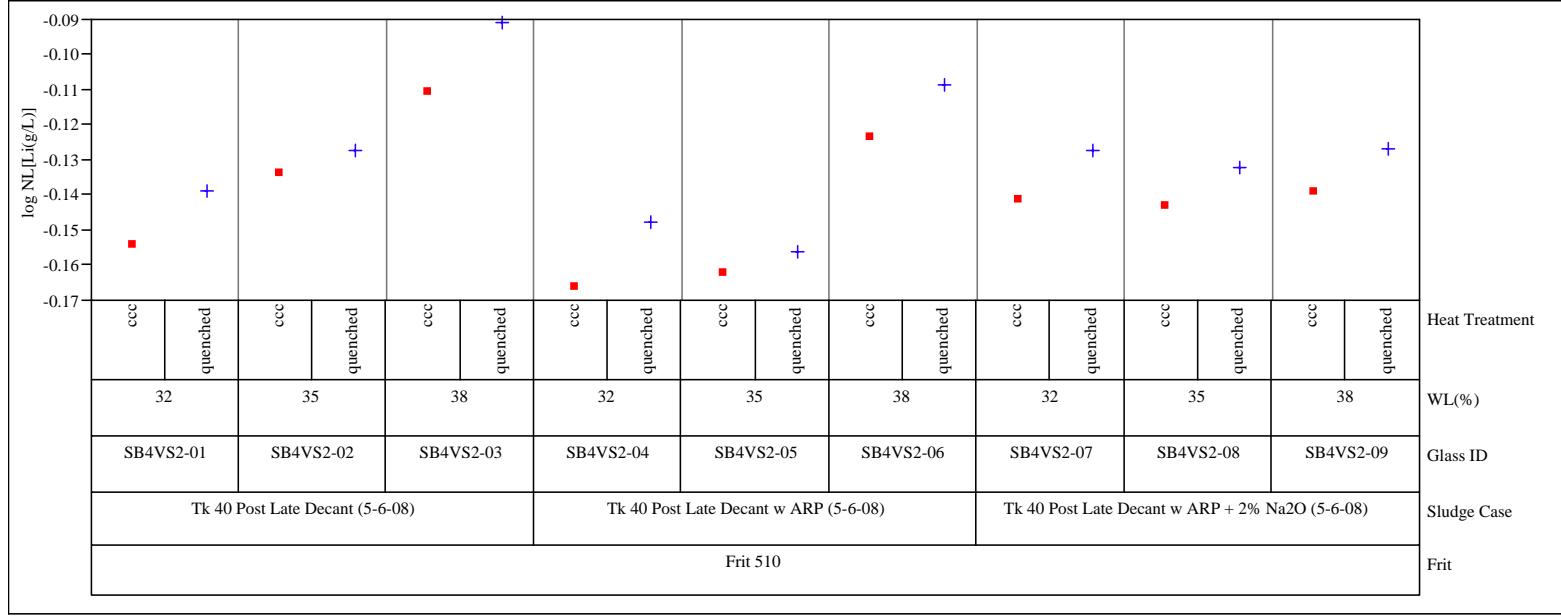


Figure B7. Effects of Heat Treatment for Study Glasses by Compositional View

Comp View $=$ measured bc

Variability Chart for log NL[Na (g/L)]

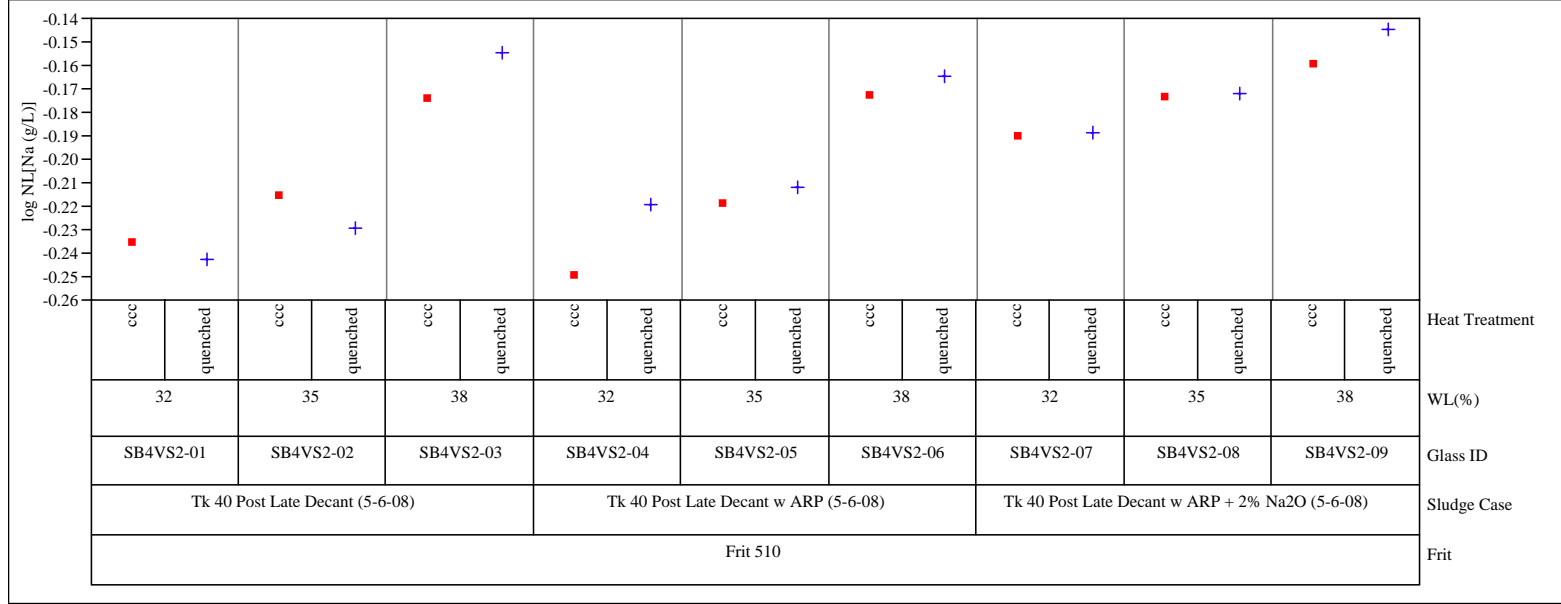

Comp View=measured bc

Variability Chart for log NL[Si (g/L)]

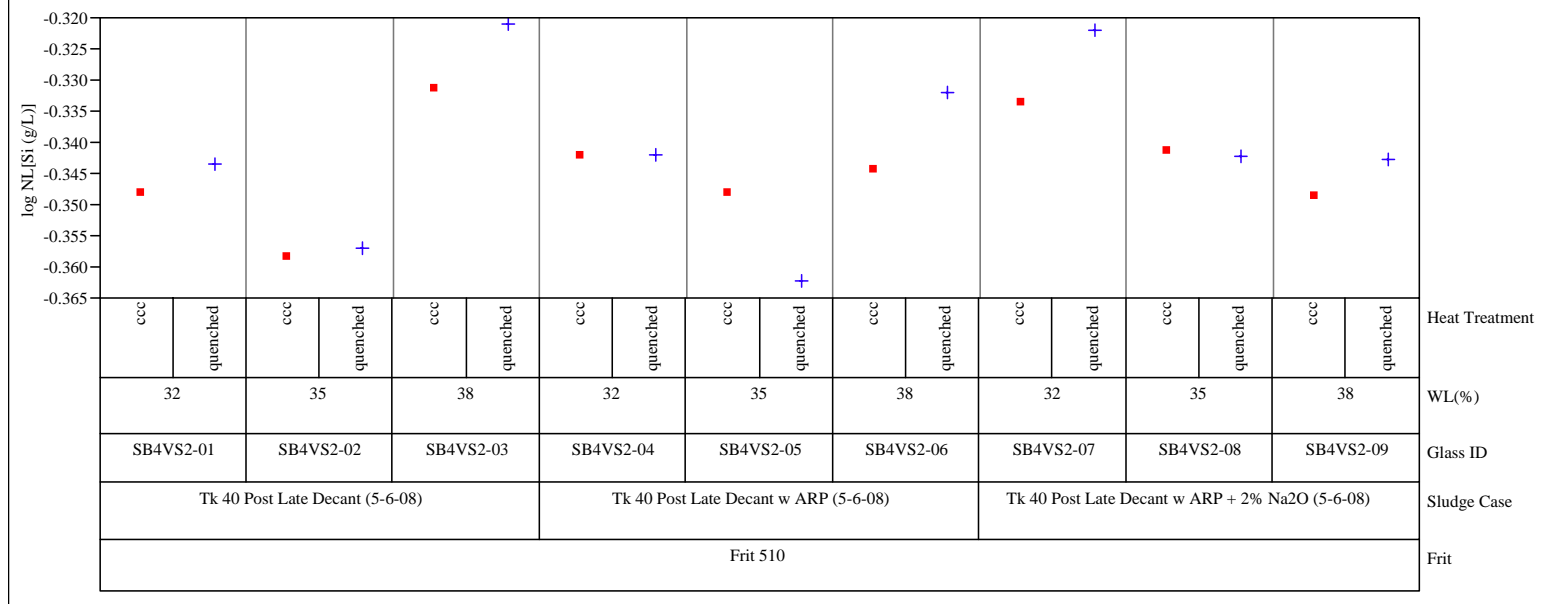

\section{Comp View=targeted}

Variability Chart for $\log$ NL[B (g/L)]

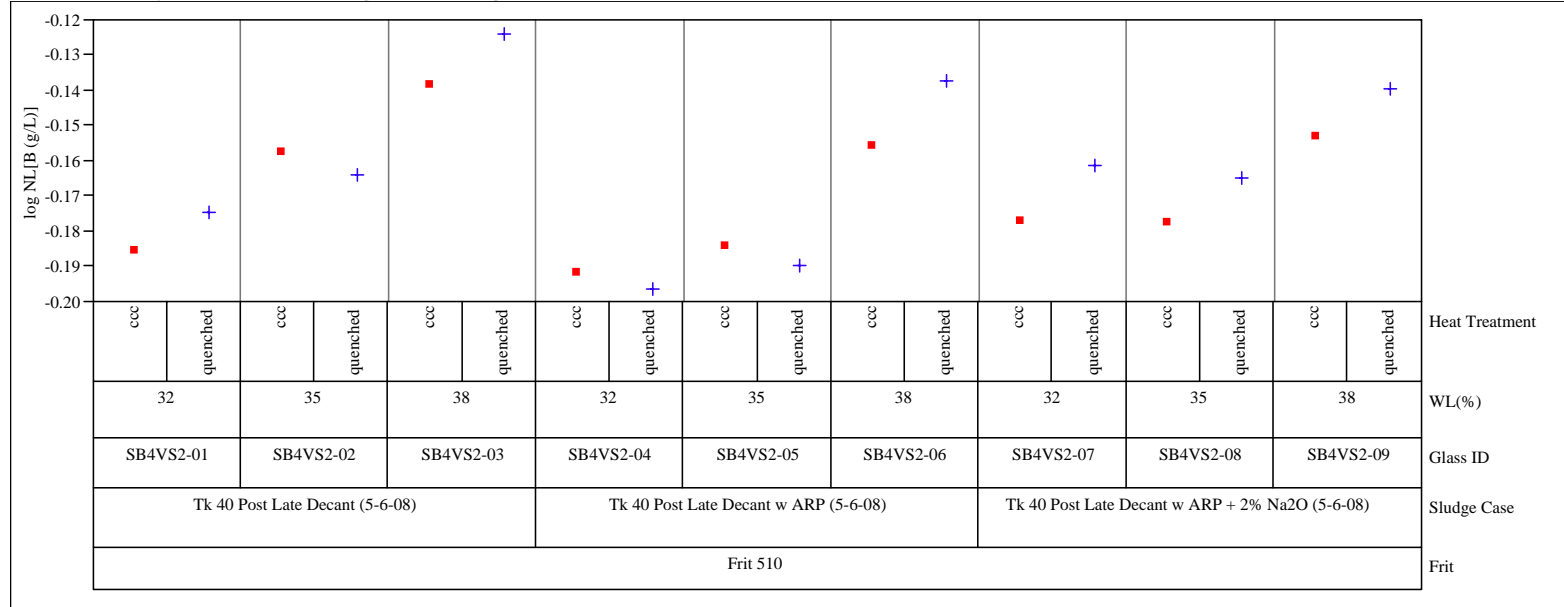


Figure B7. Effects of Heat Treatment for Study Glasses by Compositional View

\section{Comp View=targeted}

Variability Chart for $\log \mathrm{NL}[\mathrm{Li}(\mathrm{g} / \mathrm{L})]$

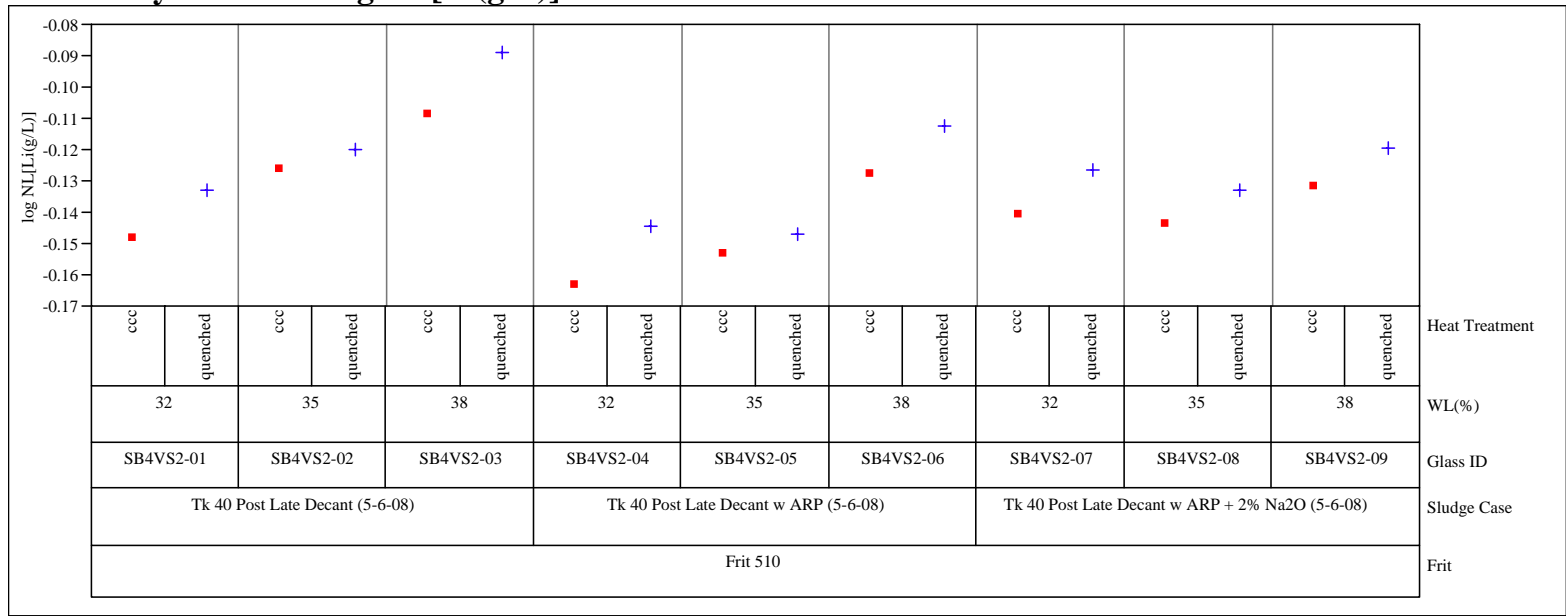

\section{Comp View=targeted}

Variability Chart for $\log \mathrm{NL}[\mathrm{Na}(\mathrm{g} / \mathrm{L})]$

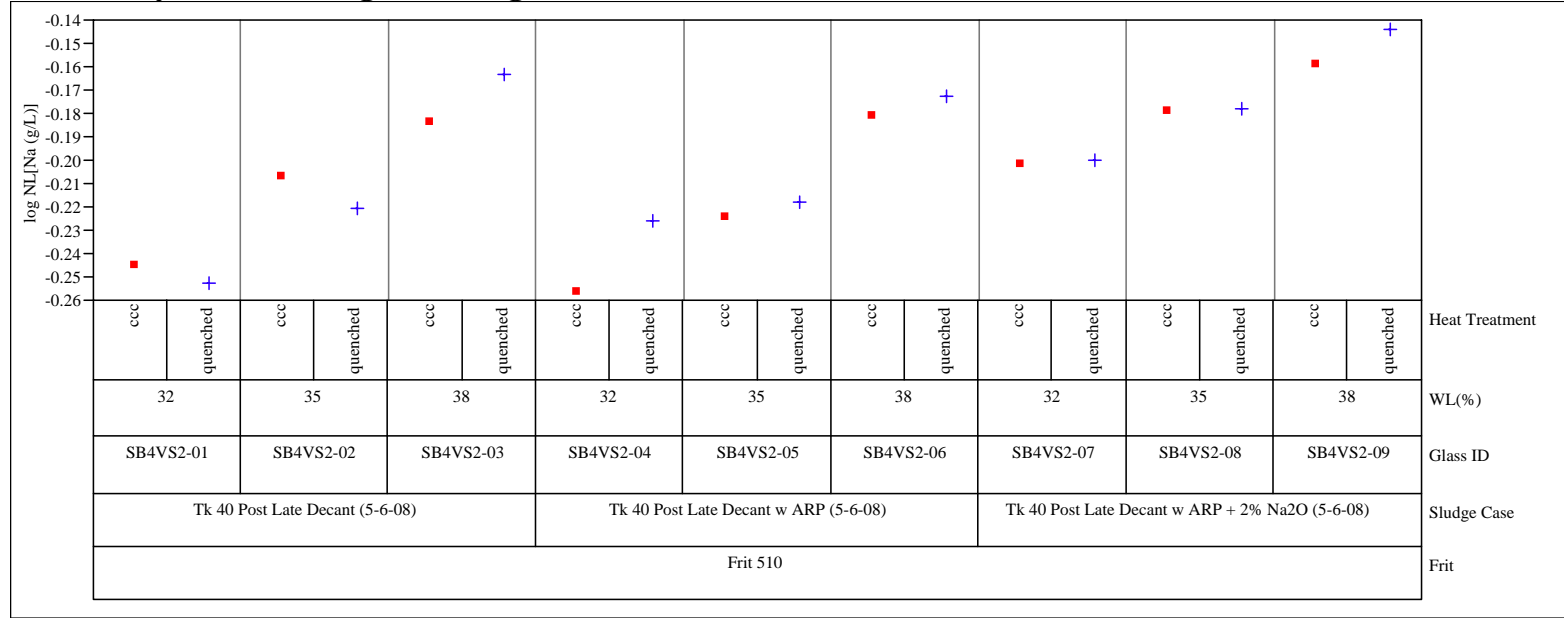

\section{Comp View=targeted}

Variability Chart for log NL[Si (g/L)]

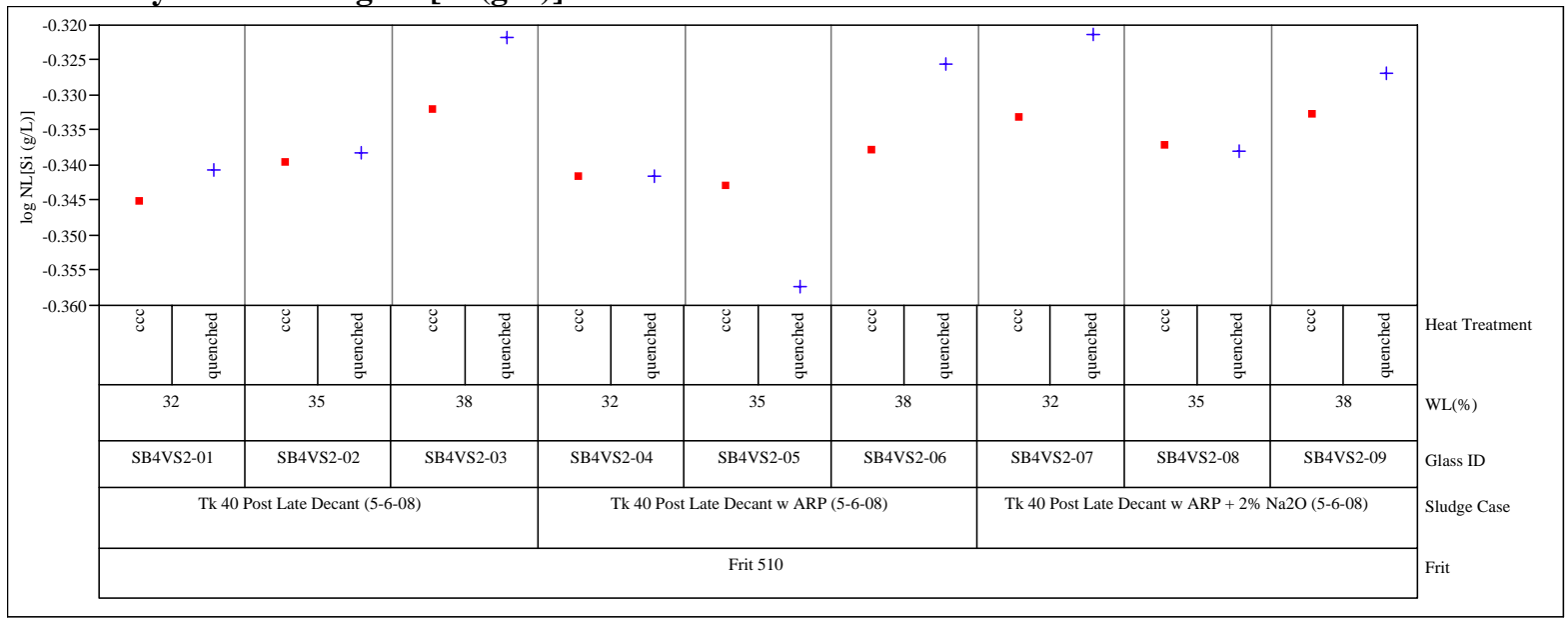




\section{Exhibit B8. del $G p\left(\Delta G_{p}\right)$ Predictions versus Common Logarithm Normalized} Leachate $(\log N L[]$.$) for B, L i, ~ N a$, and $\mathrm{Si}$ Over All Compositional Views and Heat Treatments

\begin{tabular}{|lr|l|}
\multicolumn{1}{|l|}{ Legend } & \multicolumn{2}{|c|}{ Glass Standard or Heat Treatment-Compositional View } \\
\hline$z$ & 1 & ARM \\
\hline$\diamond$ & 2 & EA \\
\hline & 3 & measured bc-cce \\
\hline & 4 & measured bc-quenched \\
\hline & 5 & measured-cce \\
\hline & 6 & measured-quenched \\
\hline 7 & 7 & targeted-cce \\
\hline & 8 & targeted-quenched \\
\hline
\end{tabular}

Bivariate Fit of $\log$ NL[B (g/L)] By del Gp

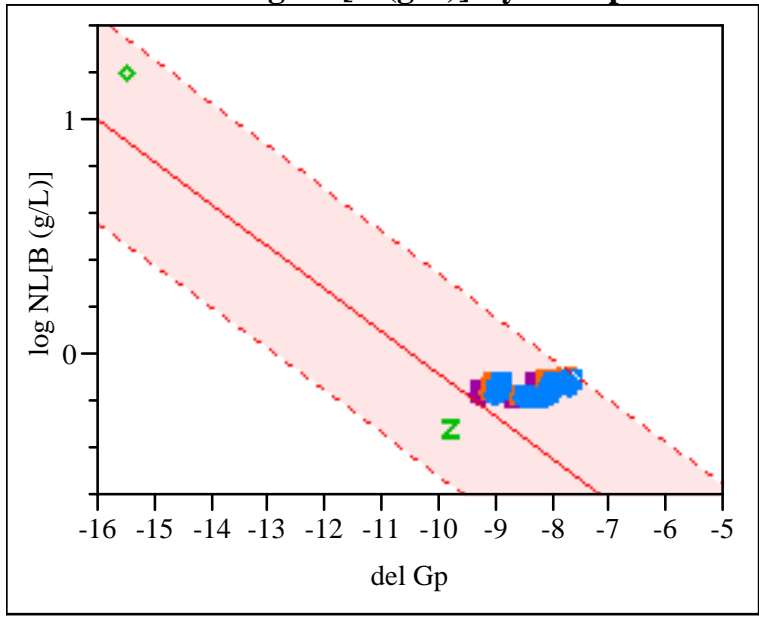

Bivariate Fit of $\log$ NL[Li (g/L)] By del Gp

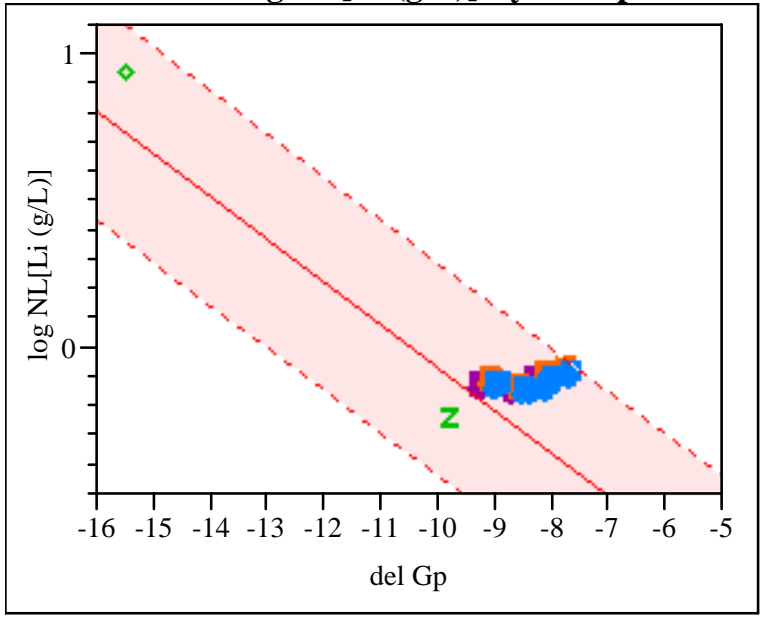

Bivariate Fit of $\log \mathrm{NL}[\mathrm{Na}(\mathrm{g} / \mathrm{L})]$ By del Gp

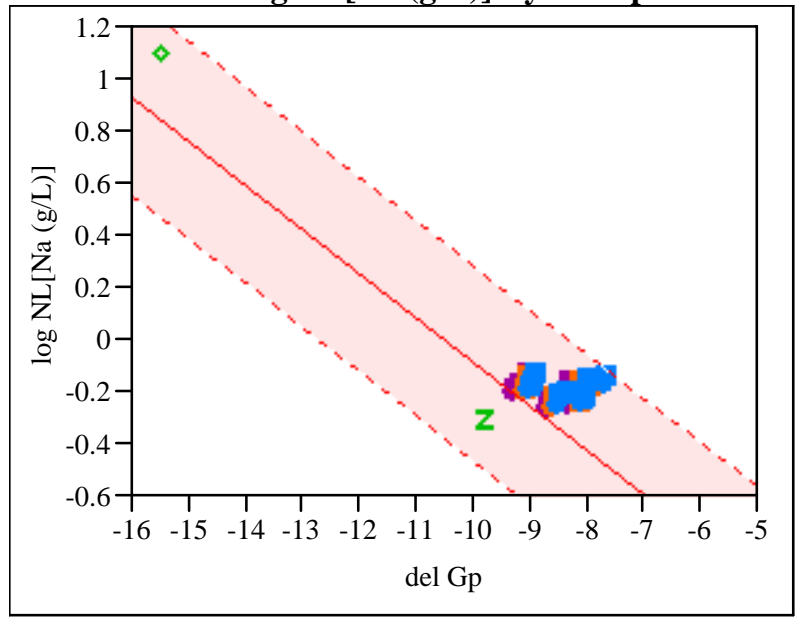

Bivariate Fit of log NL[Si (g/L)] By del Gp

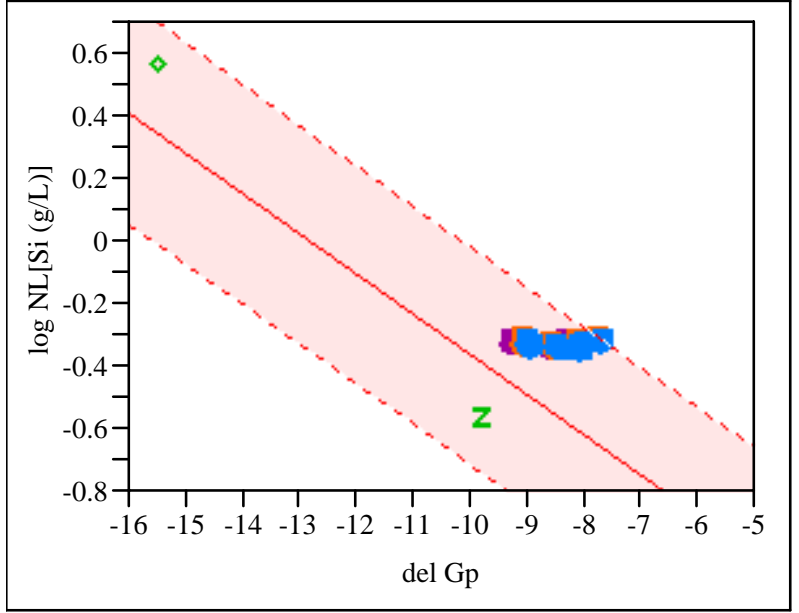




\section{Exhibit B9. del $G p\left(\Delta G_{p}\right)$ Predictions versus Common Logarithm Normalized} Leachate $(\log N L[]$.$) for B, L i, ~ N a$, and $S i$ Over All Compositional Views for Quenched Glasses

\begin{tabular}{|c|c|c|}
\hline & & Glass Standard or Heat Treatment-Compositional View \\
\hline $\mathbf{z}$ & 1 & ARM \\
\hline$\diamond$ & 2 & EA \\
\hline 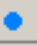 & 3 & measured bc-cce \\
\hline $\mathbf{a}$ & 4 & measured bc-quenched \\
\hline - & 5 & measured-ccc \\
\hline 口 & 6 & measured-quenched \\
\hline - & 7 & targeted-ccc \\
\hline $\mathbf{\square}$ & 8 & targeted-quenched \\
\hline
\end{tabular}

Bivariate Fit of $\log$ NL[B (g/L)] By del Gp

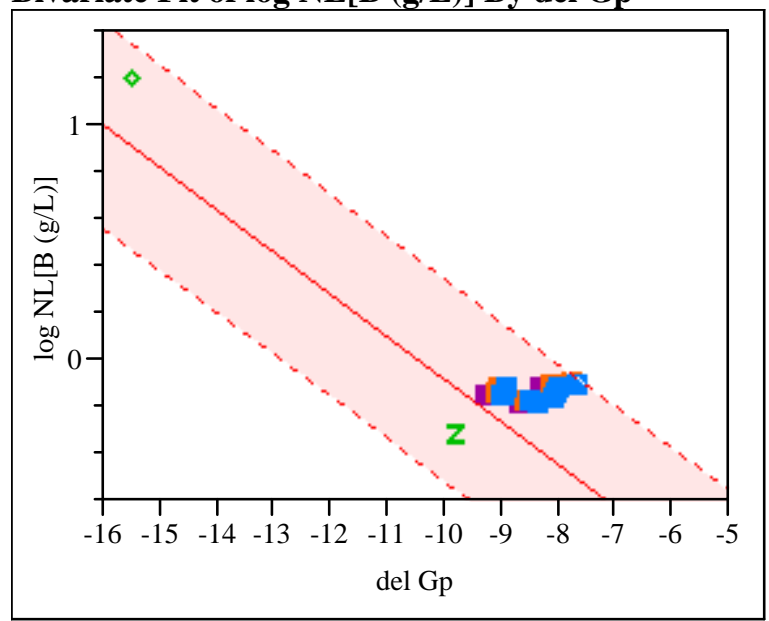

Bivariate Fit of log NL[Li (g/L)] By del Gp

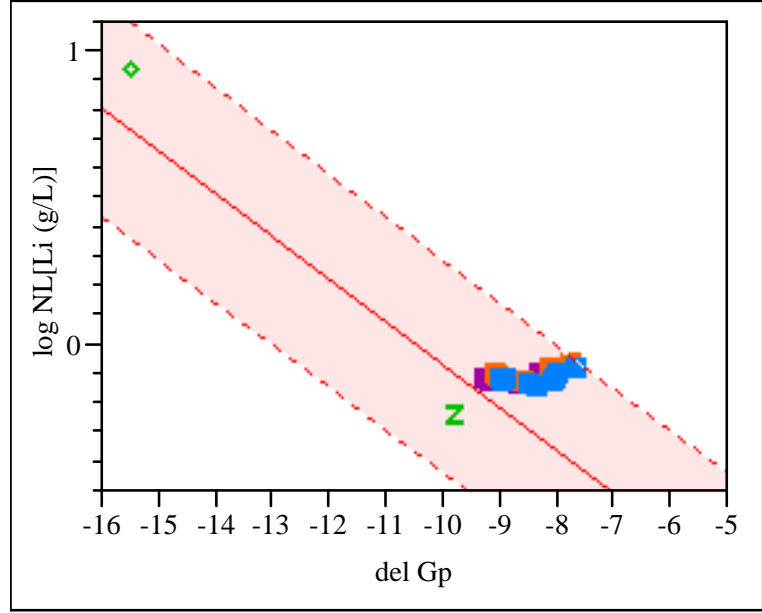

Bivariate Fit of log NL[Na (g/L)] By del Gp

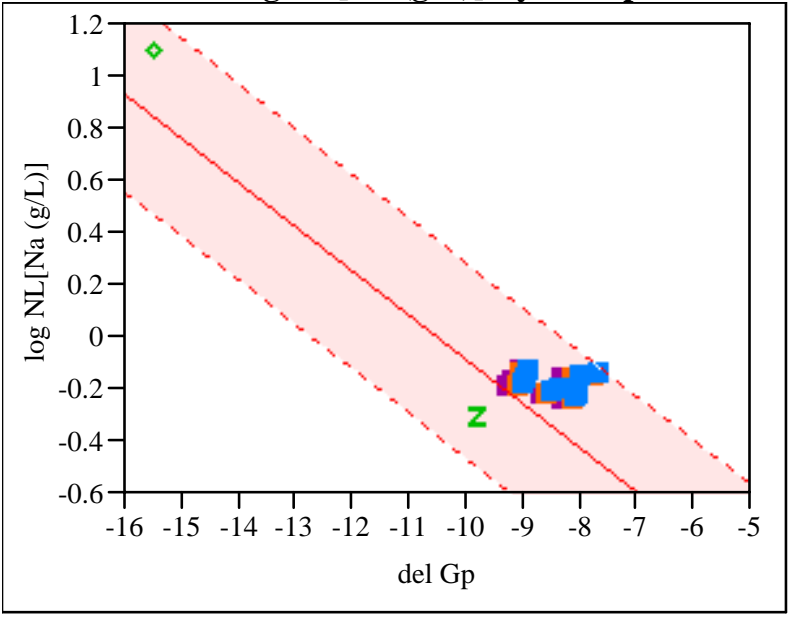

Bivariate Fit of log NL[Si (g/L)] By del Gp

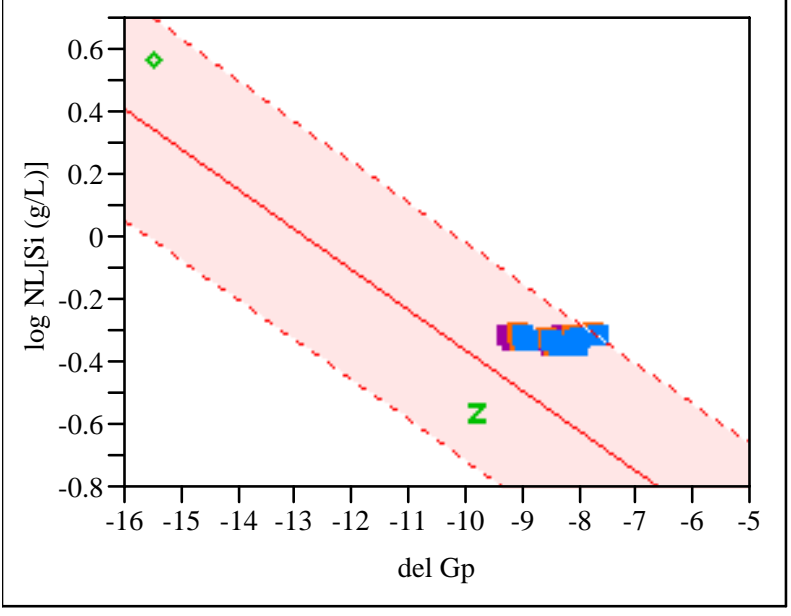


Exhibit B10. del $G p\left(\Delta G_{p}\right)$ Predictions versus Common Logarithm Normalized Leachate (log NL[.]) for $\mathrm{B}, \mathrm{Li}, \mathrm{Na}$, and $\mathrm{Si}$ Over All Compositional Views for ccc Glasses

\begin{tabular}{|c|c|c|}
\hline & & Glass Standard or Heat Treatment-Compositional View \\
\hline $\mathbf{z}$ & 1 & ARM \\
\hline$\diamond$ & 2 & EA \\
\hline$\bullet$ & 3 & measured bc-cce \\
\hline $\mathbf{\square}$ & 4 & measured bc-quenched \\
\hline - & 5 & measured-ccc \\
\hline 口 & 6 & measured-quenched \\
\hline$\bullet$ & 7 & targeted-cce \\
\hline - & 8 & targeted-quenched \\
\hline
\end{tabular}

Bivariate Fit of log NL[B (g/L)] By del Gp

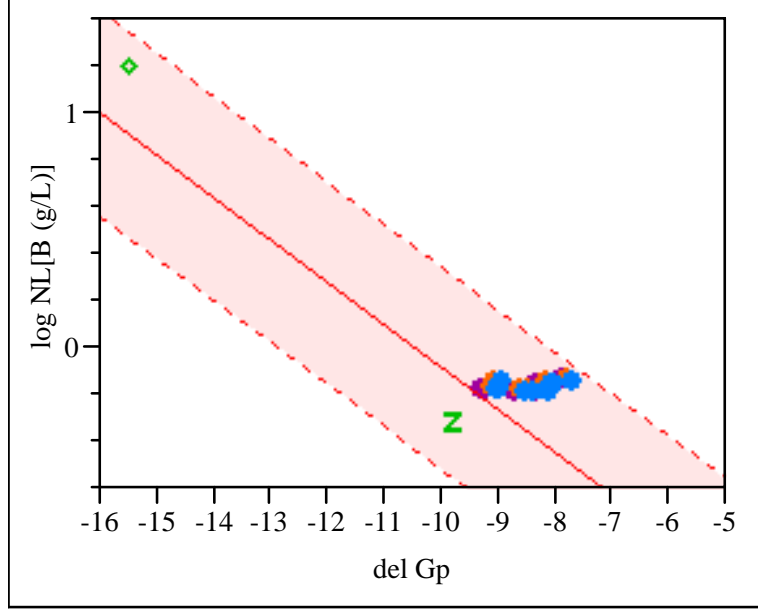

Bivariate Fit of log NL[Li (g/L)] By del Gp

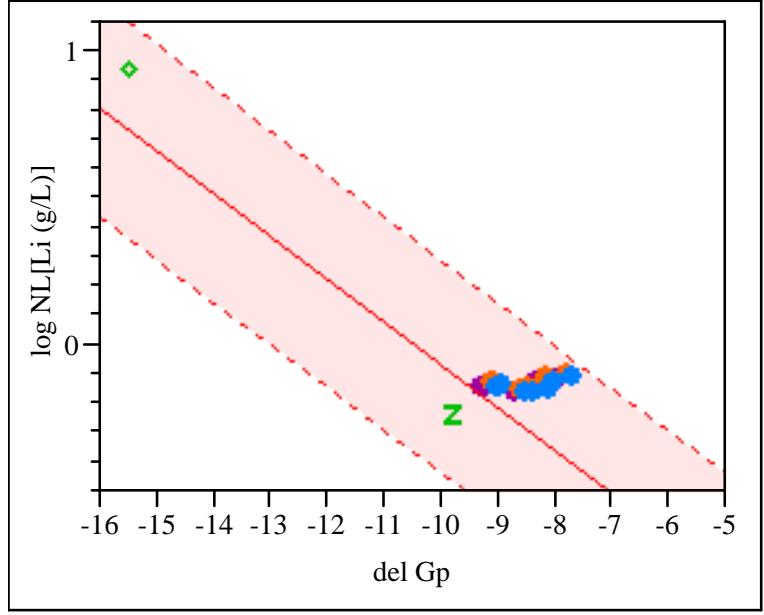

Bivariate Fit of log NL[Na (g/L)] By del Gp

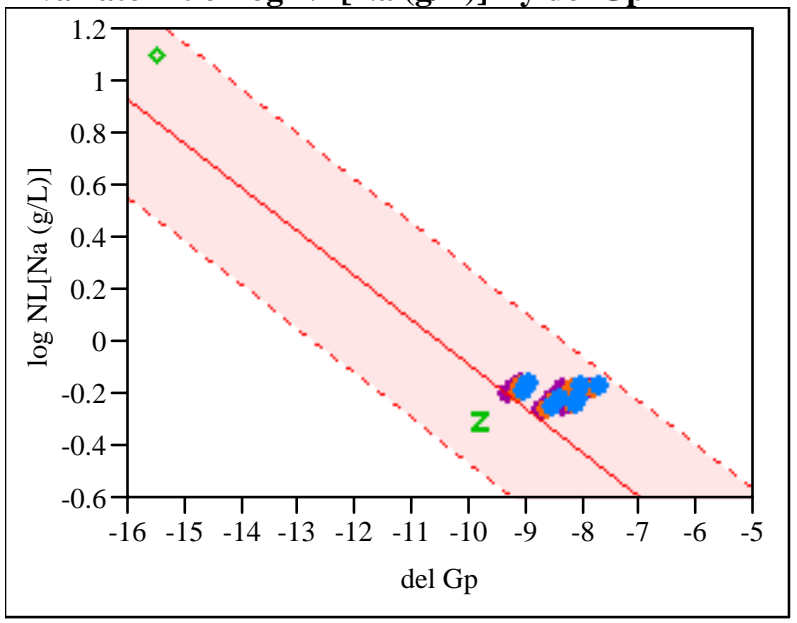

Bivariate Fit of log NL[Si (g/L)] By del Gp

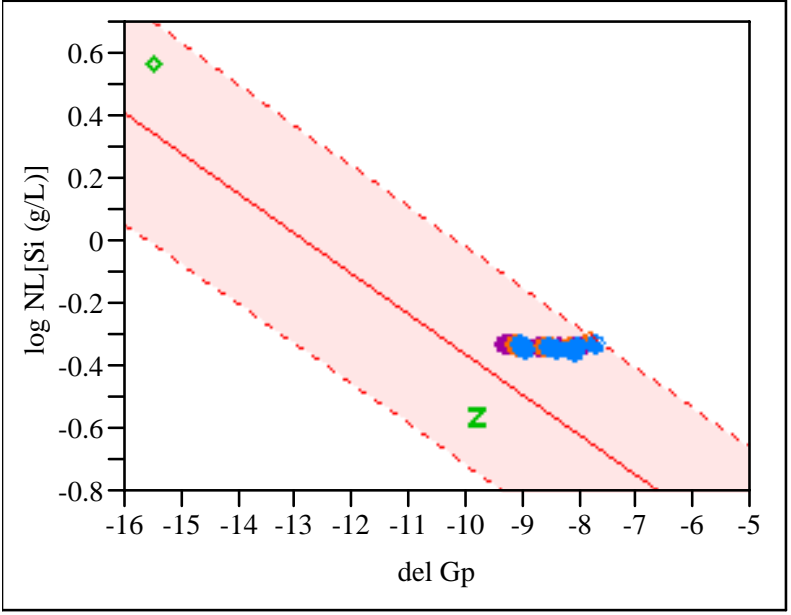




\section{Distribution:}

C.J. Bannochie, 773-42A

A.B. Barnes, 999-W

D.R. Best, 786-1A

D.B. Burns, 786-5A

B.A. Davis, 704-27S

T.B. Edwards, 999-W

H.H. Elder, 766-H

T.L. Fellinger, 704-26S

K.M. Fox, 999-W

J.M. Gillam, 766-H

J.C. Griffin, 773-A

B.A. Hamm, 766-H

C.C. Herman, 999-W

J.F. Iaukea, 704-30S

J.E. Marra, 773-A

R.T. McNew, 704-27S

T.A. Nance, 773-42A

J.D. Newell, 999-W

J.E. Occhipinti, 704-S

D.K. Peeler, 999-W

F.C. Raszewski, 999-W

J.W. Ray, 704-S

I.A. Reamer, 999-1W

H.B. Shah, 766-H

M.E. Stone, 999-W

J. Stuberfield, 766-H

M.F. Williams, 999-1W

R.J. Workman, 999-1W

A.L. Youchak, 999-W 